Sobre os produtos de Stratonovich e de Berezin de símbolos na esfera

Nazira Hanna Harb 



\title{
Sobre os produtos de Stratonovich e de Berezin de símbolos na esfera
}

\author{
Nazira Hanna Harb
}

Orientador: Prof. Dr. Pedro Paulo de Magalhães Rios

Tese apresentada ao Instituto de Ciências Matemáticas e de Computação - ICMC-USP, como parte dos requisitos para obtenção do título de Doutor em Ciências - Matemática .VERSÃO REVISADA 
Ficha catalográfica preparada pela Seção de Tratamento da Informação da Biblioteca Prof. Achille Bassi - ICMC/USP

Harb, Nazira Hanna

Sobre os produtos de Stratonovich e de Berezin de símbolos na esfera / Nazira Hanna Harb ; orientador Pedro Paulo de Magalhães Rios. -- São Carlos, 2014. $141 \mathrm{p}$.

Tese (Doutorado - Programa de Pós-Graduação em Matemática) - Instituto de Ciências Matemáticas e de Computação, Universidade de são Paulo, 2014.

1. Produto de símbolos. 2. Correspondência de símbolos. 3. Expansão assintótica. I. Rios, Pedro Paulo de Magalhães, orient. II. Título. 
A todos aqueles que me ajudaram, direta ou inderetamente, a chegar até 0 fim. 

"Tú te tornas eternamente resposável por aquilo que cativas..." (Antoine de Saint-Exupéry O pequeno príncipe.) 

Agradeço ao meu orientador Pedro P. de M. Rios pela orientação.

Um agradecimento especial ao meu marido Marcio.

Aos meus queridos amigos que sempre estiveram ao meu lado, presencialmente ou não, dando conselhos e muito apoio.

A minha família.

E a Capes pelo apoio financeiro. 

Esta tese versa sobre os produtos de Stratonovich e de Berezin de funções na esfera $S^{2}$. Cada um destes produtos é definido atravéz de uma correspondência de símbolos, que é uma aplicação linear bijetiva entre operadores lineares num espaço de Hilbert complexo de dimensão $n+1$, ou seja matrizes complexas $(n+1) \times(n+1)$, e polinômios complexos de grau próprio $\leq n$ definidos na 2-esfera, Poly $y_{\mathbb{C}}\left(S^{2}\right)_{\leq n}$, satisfazendo algumas propriedades básicas, como equivariância pela ação do grupo de rotações $S O(3)$, preservação das estruturas reais e normalização [12].

Mais geralmente, toda correspondência define um produto associativo mas não comutativo em $\operatorname{Pol} y_{\mathbb{C}}\left(S^{2}\right)_{\leq n}$ induzido do produto de operadores, chamado de produto twisted em $\operatorname{Pol} y_{\mathbb{C}}\left(S^{2}\right)_{\leq n}$. Cada um destes produtos twisted, por sua vez, pode ser escrito na forma integral

$$
f \star g(\mathbf{n})=\int_{S^{2} \times S^{2}} f\left(\mathbf{n}_{1}\right) g\left(\mathbf{n}_{2}\right) \mathbb{L}\left(\mathbf{n}_{1}, \mathbf{n}_{2}, \mathbf{n}\right) d \mathbf{n}_{1} d \mathbf{n}_{2},
$$

onde $f, g \in \operatorname{Poly}_{\mathbb{C}}\left(S^{2}\right)_{\leq n}, \mathbf{n}_{1}, \mathbf{n}_{2}, \mathbf{n} \in S^{2}$. Em tal representação integral, todas as propriedades do produto twisted são convertidas em propriedades do trikernel integral

$$
\mathbb{L}: S^{2} \times S^{2} \times S^{2} \rightarrow \mathbb{C}
$$

Os produtos twisted estudados nesta tese são os produtos induzidos pela correspondência padrão de Stratonovich e a correspondência padrão de Berezin, respectivamente, que num certo limite assintótico $2 j=n \rightarrow \infty$ definem deformações estritas da álgebra 
de Poisson de $S^{2}$ [12]. Para cada um deste dois produtos, denotados por $\star_{1}^{n}$ e $\star \frac{n}{b}$ respectivamente, seu trikernel integral é denotado por $\mathbb{L}_{1}^{j}$ e $\mathbb{L}_{\vec{b}}^{j}$, respectivamente.

A primeira parte desta tese consistiu em desenvolver fórmulas mais tratáveis para $\mathbb{L}_{1}^{j}$ e $\mathbb{L}_{\vec{b}}^{j}$ nos casos de número de spin $j=$ $1 / 2,1,3 / 2,2$, fórmulas estas escritas em termos de funções de dois e de três pontos, invariantes por $S O(3)$, como produtos escalares e determinantes.

Nossa esperança inicial era de que pudéssemos encontar padrões que nos permitissem inferir fórmulas fechadas para cada um destes trikernels, válidas para qualquer $j$, ou pelo menos que nos permitissem inferir fórmulas assintóticas para estes trikernels quando $2 j=n \rightarrow \infty$. Porém, o grau de complexidade das fórmulas desenvolvidas se mostrou fortemente crescente com $j$, frustrando nossas expectativas iniciais.

Partimos então para uma exploração preliminar de um tipo de aproximação assintótica destes produtos de certas funções oscilatórias na esfera. Mais precisamente, na segunda parte desta tese, preparamos e estudamos preliminarmente o produto de Stratonovich e o produto de Berezin (assim como o produto pontual) de dois harmônicos esféricos, $Y_{l_{1}}^{m_{1}}$ e $Y_{l_{2}}^{m_{2}} \in \operatorname{Poly}_{\mathbb{C}}\left(S^{2}\right)_{\leq n}$, no limite assintótico quando tanto $l_{1}$ como $l_{2}$ tendem a infinito linearmente com $n$ (mantendo $l_{i} \leq n$ ).

Este tipo de assintótica para tais produtos, que faz parte do que chamamos mais geralmente de "high- $l$ asymptotics", difere muito do tipo de assintótica estudada de forma detalhada em [12], na qual $n \rightarrow \infty$, mas $l_{1}$ e $l_{2}$ são mantidos finitos. Então, a partir de um exemplo particular para nossa exploração preliminar, levantamos uma conjectura sobre como estes produtos se comparam no limite assintótico quando $l_{1}$ e $l_{2}$ tendem para infinito linearmente com o número de spin $j$. 
This thesis is about the Stratonovich and the Berezin products of functions on the 2-sphere. Each one of these products is defined via a spin- $j$ symbol correspondence, a linear bijective map from the space of operators on an $(n+1)$-dimensional complex Hilbert Space, i.e. $(n+1) \times(n+1)$ complex matrices, and the space of complex polynomials on $S^{2}$ of proper degree $\leq n$, denoted Poly $_{\mathbb{C}}\left(S^{2}\right)_{\leq n}$, satisfying certain basic properties like equivariance under the action of $S O(3)$, preservation of real structures and normalization [12].

More generally, every spin- $j$ symbol correspondence defines an associative noncommutative product on $\operatorname{Poly}_{\mathbb{C}}\left(S^{2}\right)_{\leq n}$ induced from the operator product, which is called a twisted product on Poly $_{\mathbb{C}}\left(S^{2}\right)_{\leq n}$. Each twisted product can be written in integral form as

$$
f \star g(\mathbf{n})=\iint_{S^{2} \times S^{2}} f\left(\mathbf{n}_{1}\right) g\left(\mathbf{n}_{2}\right) \mathbb{L}\left(\mathbf{n}_{1}, \mathbf{n}_{2}, \mathbf{n}\right) d \mathbf{n}_{1} d \mathbf{n}_{2},
$$

where $f, g \in \operatorname{Poly}_{\mathbb{C}}\left(S^{2}\right)_{\leq n}, \mathbf{n}_{1}, \mathbf{n}_{2}, \mathbf{n} \in S^{2}$. In such an integral representation, all properties of the twisted product are translated to properties of its integral trikernel

$$
\mathbb{L}: S^{2} \times S^{2} \times S^{2} \rightarrow \mathbb{C}
$$

The twisted products studied in this thesis are the ones obtained via the standard Stratonovich-Weyl and the standard Berezin symbol correspondences, respectively, which in a certain asymptotic limit $2 j=n \rightarrow \infty$ define strict deformation quantizations of $S^{2}$, i.e. strict deformations of the Poisson algebra of $S^{2}$ [12]. For 
each of these two products on Poly $y_{\mathbb{C}}\left(S^{2}\right)_{\leq n}$, denoted by $\star_{1}^{n}$ and $\star \frac{n}{b}$ respectively, its integral trikernel is denoted by $\mathbb{L}_{1}^{j}$ and $\mathbb{L}_{\vec{b}}^{j}$, respectively.

In the first part of this thesis, we obtained better formulae for these trikernels, for values of spin number $j=1 / 2,1 / 3 / 1,2$. These formulas are written in terms of $S O(3)$-invariant functions of two and three points on $S^{2}$, like scalar products and determinants.

We initially hoped to be able to obtain closed formulae for these trikernels which would be valid for every $j$, or at least be able to infer asymptotic formulas for these trikernels when $2 j=n \rightarrow \infty$. However, the degree of complexity of the formulae we have obtained increases strongly with $j$, frustrating our initial expectations.

We thus started on a preliminary investigation of a kind of asymptotic approximation for these products of certain oscillatory functions on the sphere. More precisely, in the second part of this thesis, we prepared and preliminarily studied the Stratonovich product and the Berezin product (as well as the pointwise product) of spherical harmonics $Y_{l_{1}}^{m_{1}}$ and $Y_{l_{2}}^{m_{2}} \in \operatorname{Poly}_{\mathbb{C}}\left(S^{2}\right)_{\leq n}$, in the asymptotic limit when $l_{1}$ and $l_{2}$ tend to infinity linearly with $n$ (keeping $l_{i} \leq n$ ).

This kind of asymptotics for these products, belonging to what we more generally call "high- $l$ asymptotics", differs drastically from the kind of asymptotics studied in detail in [12], in which $n \rightarrow$ $\infty$ but $l_{1}$ and $l_{2}$ are kept finite. Then, based on a particular example of our preliminary exploration, we advanced a conjecture on how these products behave and compare with each other, in the asymptotic limit when $l_{1}$ and $l_{2}$ tend to infinity linearly with the spin number $j$. 
1 Preliminares 1

1.1 Cinemática das rotações. . . . . . . . . . . . . . . . . . . . . . . . 1

1.1.1 Propriedades das Rotações . . . . . . . . . . . . . . . . . . . . . . 2

1.1 .2 Relação entre $S U(2)$ e $S O(3) \ldots \ldots$. . . . . . . . . . . . . . . . . . . . . .

1.1.3 Parametrizações do grupo de rotações . . . . . . . . . . . . . . . . . 8

1.2 Momento angular e harmônicos esféricos . . . . . . . . . . . . . . . . 13

1.3 Os multiplets do momento angular . . . . . . . . . . . . . . 21

1.4 Coeficientes de Wigner e 3j simbolo. . . . . . . . . . . . . . . . 24

1.5 Coeficientes de Racah e 6j-simbolo. . . . . . . . . . . . . . . 30

2 Os trikernels de Stratonovich e de Berezin 33

2.1 Correspondência de símbolos para sistemas de spin. . . . . . . . . . . . 34

2.1.1 Correspondência de símbolos via operador kernel, o operador kernel de Stratonovich-Weyl e de Berezin. . . . . . . . . . . . . . 37

2.2 Produto Twisted na Esfera . . . . . . . . . . . . . . . . 41

2.3 Cálculos explícitos para o trikernel de Stratonovich e de Berezin. . . . 47

2.3.1 Cálculos explícitos para $j=\frac{1}{2}, 1, \frac{3}{2}, 2 \ldots \ldots \ldots \ldots$

3 Exploração preliminar das assintóticas para os produtos de Stratonovich de Berezin de funções fortemente oscilatórias $\quad 65$

3.1 Exploração assintótica dos produtos twisted padrão. . . . . . . . . . . . 67

3.1.1 Preparando o produto de Stratonovich padrão para a exploração assintótica . . . . . . . . . . . . . . . 70

3.1.2 Preparando o produto de Berezin padrão para a exploração assintótica ......................... 73 
3.1.3 Expressão assintótica do produto pontual . . . . . . . . . . . 77

3.1.4 Exemplo para $\lambda_{1}$ e $\lambda_{2}$ particulares . . . . . . . . . . . . 80

3.2 Conclusão . . . . . . . . . . . . . . . . . . . . . . . . . . . . . 81

Apêndice A: contas Stratonovich 83

$\begin{array}{ll}\text { Apêndice B: Tetraedro } & 97\end{array}$

Apêndice C: detalhes do exemplo 3.1.4 101

3.3 Cálculo da aproximação para o produto de Stratonovich . . . . . . . . 101

3.4 Cálculo da aproximação para o produto de Berezin . . . . . . . . . . . 119

3.5 Cálculo da aproximação para o produto pontual . . . . . . . . . . . . . 132

3.6 O método de "Steepest descents" . . . . . . . . . . . . . . . . . 133

Apêndice D: Software Mathematica $\quad 137$

$\begin{array}{lr}\text { Referências Bibliográficas } & 137\end{array}$ 
O objetivo dessa tese, primeiramente, era encontrar uma fórmula fechada para os trikernels integrais que definem o produto de Stratonovich e o produto de Berezin, cujas breves definições daremos a seguir. Como o objetivo inicial não foi atingido, partimos para uma exploração assintótica preliminar desses produtos, tomando como base o trabalho de [12].

Lembramos que os produtos de Stratonovich e de Berezin de funções na esfera $S^{2}$ são casos particulares de produtos definido atravéz de uma correspondência de símbolos, que é uma aplicação linear bijetiva entre operadores lineares num espaço de Hilbert complexo de dimensão $n+1$, ou seja matrizes complexas $(n+1) \times(n+$ 1 ), e polinômios complexos de grau próprio $\leq n$ definidos na 2-esfera, $\operatorname{Pol} y_{\mathbb{C}}\left(S^{2}\right)_{\leq n}$, satisfazendo algumas propriedades básicas, como equivariância pela ação do grupo de rotações $S O(3)$, preservação das estruturas reais e normalização [12].

Mais geralmente, toda correspondência de símbolos, como descrita acima, define um produto associativo mas não comutativo em $\operatorname{Pol} y_{\mathbb{C}}\left(S^{2}\right)_{\leq n}$ induzido do produto de operadores, chamado de produto twisted em $\operatorname{Pol}_{\mathbb{C}}\left(S^{2}\right)_{\leq n}$. Os produtos twisted estudados nesta tese são os produtos induzidos pela correspondência padrão de Stratonovich e a correspondência padrão de Berezin, respectivamente, que num certo limite assintótico $2 j=n \rightarrow \infty$ definem deformações estritas da álgebra de Poisson de $S^{2}$ [12].

Os trikernels de Stratonovich e de Berezin aparecem quando se define tais produtos twisted de duas funções na esfera, da seguinte forma:

$$
f \star g(n)=\iint_{S^{2} \times S^{2}} f\left(n_{1}\right) g\left(n_{2}\right) \mathbb{L}\left(n_{1}, n_{2}, n\right) d n_{1} d n_{2},
$$


onde $f, g \in \operatorname{Poly}_{\mathbb{C}}\left(S^{2}\right)_{\leq n}$ e $n_{1}, n_{2}, n$ são pontos em $S^{2}$. Em tal representação integral, todas as propriedades do produto twisted são convertidas no trikernel integral

$$
\mathbb{L}: S^{2} \times S^{2} \times S^{2} \rightarrow \mathbb{C}
$$

Para cada um dos trikernels, que estudaremos, suas notações serão: $\mathbb{L}_{1}^{j}$ e $\mathbb{L}_{\vec{b}}^{j}$, os trikernels de Stratonovich e Berezin, respectivamente.

Inicialmente as definições para esses trikernels são:

$$
\begin{gathered}
L_{1}^{j}\left(n_{1}, n_{2}, n\right)=\sum_{l_{k}, m_{k}} \frac{(-1)^{n} \sqrt{(2 j+1)\left(2 l_{1}+1\right)\left(2 l_{2}+1\right)(2 l+1)}}{(4 \pi)^{2}} \\
{\left[\begin{array}{ccc}
l_{1} & l_{2} & l \\
m_{1} & m_{2} & -m
\end{array}\right][j] \overline{Y_{l_{1}}^{m_{1}}}\left(n_{1}\right) \overline{Y_{l_{2}}^{m_{2}}}\left(n_{2}\right) \overline{Y_{l}^{m}}(n)}
\end{gathered}
$$

e

$$
\begin{gathered}
L_{\vec{b}}^{j}\left(n_{1}, n_{2}, n\right)=\sum_{l_{k}, m_{k}} \frac{(-1)^{n} \sqrt{(2 j+1)\left(2 l_{1}+1\right)\left(2 l_{2}+1\right)(2 l+1)}}{(4 \pi)^{2}} \\
{\left[\begin{array}{ccc}
l_{1} & l_{2} & l \\
m_{1} & m_{2} & -m
\end{array}\right][j] \sqrt{\frac{\left(\begin{array}{c}
n+l_{1}+1 \\
l_{1}
\end{array}\right)\left(\begin{array}{c}
n+l_{2}+1 \\
l_{2}
\end{array}\right)\left(\begin{array}{c}
n \\
l_{3}
\end{array}\right)}{\left(\begin{array}{c}
n \\
l_{1}
\end{array}\right)\left(\begin{array}{l}
n \\
l_{2}
\end{array}\right)\left(\begin{array}{c}
n+l_{3}+1 \\
l_{3}
\end{array}\right)}\left(n_{1}\right) \overline{Y_{l_{2}}^{m_{2}}}\left(n_{2}\right) \overline{Y_{l}^{m}}(n),}}
\end{gathered}
$$

onde,

$$
\left[\begin{array}{ccc}
l_{1} & l_{2} & l \\
m_{1} & m_{2} & -m
\end{array}\right][j]=\left\{\begin{array}{ccc}
l_{1} & l_{2} & l \\
j & j & j
\end{array}\right\} \cdot\left(\begin{array}{ccc}
l_{1} & l_{2} & l \\
-m_{1} & -m_{2} & m
\end{array}\right)
$$

que é o produto de $6 j$-símbolo pelo $3 j$-símbolo de Wigner, respectivamente, vide [12].

A idéia inicial de nosso estudo era encontrar fórmulas fechadas, escritas em termos de produtos internos e determinantes, para ambos esses trikernels $\mathbb{L}_{1}^{j}$ e $\mathbb{L}_{\vec{b}}^{j}$ que fossem válidas para todo $j$, ou pelo menos que nos permitissem inferir fórmulas assintóticas para estes trikernels, quando $n \rightarrow \infty$. Porém tais objetivos iniciais não foram atingidos, porque a complexidade das fórmulas que obtivemos cresce muito com $j$. Ainda assim, conseguimos obter uma fórmula alternativa para esses trikernels, como mostrado no Capítulo 2, que nos permitiu calcular mais alguns exemplos explícitos para estes trikernels, que não são encontrados na literatura. O exemplo que pode ser encontrado 
na literatura é um exemplo para o trikernel de Stratonovich para $j=1 / 2$ em [20]. Aqui, nesse trabalho calculamos, exemplos explícitos, que estão na seção 2.3.1, para os trikernels de Stratonovich e de Berezin para $j=1, \frac{3}{2}, 2$. Exemplos esses calculados a mão, já que não conseguimos nenhuma ferramenta que arrumasse essas contas de maneira a nos fornecer a tal fórmula procurada.

Após esse objetivo inicial, partimos para um estudo assintótico dos produtos de Stratonovich e de Berezin de harmônicos esféricos. Produtos estes definidos da seguinte maneira [12]:

$Y_{l_{1}}^{m_{1}} *_{1} Y_{l_{2}}^{m_{2}}=(-1)^{2 j+m} \sum_{l_{3}=0}^{2 j} \sqrt{(2 j+1)\left(2 l_{1}+1\right)\left(2 l_{2}+1\right)(2 l+1)} \cdot\left[\begin{array}{ccc}l_{1} & l_{2} & l \\ m_{1} & m_{2} & -m\end{array}\right][j] Y_{l}^{m}$

e

$Y_{l_{1}}^{m_{1}} * \vec{b} Y_{l_{2}}^{m_{2}}=(-1)^{2 j+m} \sum_{l_{3}=0}^{2 j} \sqrt{(2 j+1)\left(2 l_{1}+1\right)\left(2 l_{2}+1\right)(2 l+1)} \cdot\left[\begin{array}{ccc}l_{1} & l_{2} & l \\ m_{1} & m_{2} & -m\end{array}\right][j] \frac{b_{l}^{n}}{b_{l_{1}}^{n} b_{l_{2}}^{n}} Y_{l}^{m}$,

respectivamente.

Nosso estudo, na verdade, constitui uma exploração preliminar para saber o que aconteceria no limite semiclássico do produto twisted (de Stratonovich padrão e de Berezin padrão) de funções fortemente oscilatórias na esfera. Na monografia [12], foi feito uma análise assintótica desses produtos e a dinâmica em primeira ordem é dada pelo parênteses de Poisson. Porém, o limite assintótico destes produtos de harmônicos esféricos, que foi estudado em detalhe em [12], é o limite quando $n \rightarrow \infty$, mas $l_{1}$ e $l_{2}$ são mantidos finitos. O que fizemos aqui, descrito no Capítulo 3, foi tomarmos $l_{1}, l_{2} \rightarrow \infty$ linearmente com $n \rightarrow \infty$, e verificar preliminarmente o que acontece com estes produtos em tal limite assintótico.

No Capítulo 3, portanto, avançamos uma conjectura, a ser melhor estudada em trabalhos futuros, que a tiramos a partir de um exemplo particular desta exploração assintótica dos produtos citados. Nesta conjectura, ambos os produtos tendem a zero em primeira ordem (coincidindo com o produto pontual nesta aproximação), mas o produto de Berezin tende a zero mais rapidamente do que o produto de Stratonovich. Um melhor entendimento desta conjectura e de suas possíveis consequências para a dinâmica semiclássica de funções oscilatórias na esfera, ficando, portando, para trabalhos futuros.

Detalhando os próximos capítulos que estão na tese, iniciamos com o capítulo "Preliminares"onde há uma breve introduçao dos principais tópicos necessários para o entendimento desse trabalho. 
Depois, no capitulo 2, "Trikernel de Stratonovich e Berezin"introduzimos o que vem a ser ambos os trikernels e de onde provêem. Desenvolvendo a fórmula, que se verá não ser fechada, porém ainda dependente de produtos internos e determinantes. Ainda nesse capítulo, foram cálculados exemplos, que não se encontram na literatura, como $j=1,3 / 2$ e 2 . Não conseguiu-se obter uma generalidade nem um padrão a partir desses exemplos, mas valem como exemplos inéditos e ponto de partida para possíveis explorações futuras.

No capítulo 3, "Exploração preliminar das assíntotas para os produtos de Stratonovich e Berezin", desenvolve-se os produtos basenado-se em expansões assintóticas de Ponzano-Regge, [17], obtemos integrais as quais não se conseguiu resolver, mesmo através de sistemas algébricos computacionais como Mathematica, Maple e Matlab. Fizemos então casos particulares para buscar pistas e até poder formar uma conjectura sobre essa expansão assintótica inicial. Talvez, com mais tempo de estudo, seja possível desenvolver essas assíntoticas de uma maneira mais algébrica e geral.

Nos Apêndices A e B , estão as contas detalhadas, omitidas nos capítulos 1 e 2.

No Apêndice C, estão os detalhes do exemplo 3.1.2 e uma breve apresentação do que é o método de "steepest descents"usado para a resolução desse mesmo exemplo.

No Apêndice D, estão algumas das contas feitas no programa Mathematica. 


\begin{tabular}{l|c|}
\cline { 2 - 2 } & Capítulo \\
\cline { 2 - 2 } & 1 \\
1
\end{tabular}

Preliminares

O Objetivo desse capítulo é fazer um apanhado geral de alguns tópicos usados para o entendimento dessa tese. Para um conhecimento mais profundo desses tópicos consultar [8], [5] e [14].

\subsection{Cinemática das rotações.}

Pelo termo "cinemática das rotações"entendemos como a descrição de objetos físicos sob rotações. Apesar da aparente simplicidade desse assunto, a cinemática das rotações provará ter algumas sutilezas.

O elemento essencial na descrição de um sistema físico está no modelo matemático ao espaço que está submetido. Essa associação é postulada. Começemos com os conceitos postulados da relatividade Newtoniana. O modelo matemático a ser associado a essa física Newtoniana é aquela em que um ponto físico (massa) é identificado com pontos pertencentes a um espaço tri-dimensional Euclideano, E(3). Onde tri-dimensional significa que um ponto é uma tripla ordenada, com cada coordenada pertencente aos reais. E espaço euclideano significa que sob simetrias espaciais todas as distâncias entre pontos são preservadas, portanto, todos os comprimentos e ângulos serão preservados também. As simetrias no espaço Euclideano podem ser compostas a partir de sime- 
trias especiais: translações, rotações e inversões que deixam um ponto fixo. Rotações preservam orientações e as inversões as ivertem.

\subsubsection{Propriedades das Rotações}

É conhecido o fato de que numa simetria Euclideana que deixa um ponto fixo, deixa todos os pontos de uma reta, por esse ponto, fixos. A rotação é uma simetria Euclideana caracterizada por um eixo (denotado por um vetor unitário $\widehat{n}$ ), um ângulo de rotação $\phi$ e um senso de rotação em torno do eixo (tomado sempre como a regra da mão direita). Denotamos uma rotação por $\mathfrak{R}(\phi, \widehat{n})$.

Por considerações geometricas, podemos obter um resultado geral para a transformação de um vetor dado, $\vec{r}$, sob uma rotação (deixando a origem fixada). A componente de $\vec{r}$ ao longo de $\widehat{n}$ deve se manter a mesma: $<\widehat{n}, \vec{r}>\vec{n} \rightarrow<\widehat{n}, \vec{r}>\widehat{n}$. A componente perpendicular $\vec{r}-<\widehat{n}, \vec{r}>\widehat{n}$, rotaciona por ângulo $\phi$ e, como qualquer outro vetor perpendicular a $\widehat{n}$, pode ser expressado, depois da rotação, como uma combinação linear de $\vec{r} \times \widehat{n}$ e $\vec{r}-<\widehat{n}, \vec{r}>\widehat{n}$. Encontramos:

$$
\vec{r}-<\widehat{n}, \vec{r}>\widehat{n} \rightarrow[\vec{r}-<\widehat{n}, \vec{r}>\widehat{n}] \cos (\phi)+(\widehat{n} \times \vec{r}) \operatorname{sen}(\phi)
$$

tal que

$\mathfrak{R}(\phi, \widehat{n}): \vec{r} \rightarrow \overrightarrow{r^{\prime}}=\mathfrak{R}(\phi, \widehat{n}) \vec{r}=\vec{r} \cos (\phi)+\widehat{n}<\widehat{n}, \vec{r}>(1-\cos (\phi))+(\widehat{n} \times \vec{r}) \sin (\phi)$.

Esse resultado pode ser usado para obter as características vetoriais de uma rotação. Portanto verificamos, para vetores $\widehat{p}$ e $\widehat{q}$ unitários perpendiculares a $\widehat{n}$ tais que $(\widehat{n}, \widehat{p}, \widehat{q})$ constituem uma triad de mão direita de vetores perpendiculares, que:

$$
\mathfrak{R}(\phi, \widehat{n})(\widehat{p}+i \widehat{q})=e^{-i \phi}(\widehat{p}+i \widehat{q})
$$

De fato, $\mathfrak{R}(\phi, \widehat{n})(\widehat{p}+i \widehat{q})=\mathfrak{R}(\phi, \widehat{n}) \widehat{p}+i \mathfrak{R}(\phi, \widehat{n}) \widehat{q}=\widehat{p} \cos (\phi)+\widehat{n}<\widehat{n}, \widehat{p}>(1-\cos (\phi))+(\widehat{n} \times$ $\widehat{p}) \sin (\phi)+i(\widehat{q} \cos (\phi)+\widehat{n}<\widehat{n}, \widehat{q}>(1-\cos (\phi))+(\widehat{n} \times \widehat{q}) \sin (\phi))=\widehat{p} \cos (\phi)+(\widehat{n} \times \widehat{p}) \operatorname{sen}(\phi)+$ $\widehat{q} \cos (\phi)+(\widehat{n} \times \widehat{q}) \operatorname{sen}(\phi)=\widehat{p} \cos (\phi)+\widehat{q} \operatorname{sen}(\phi)+i \widehat{q} \cos (\phi)-i \widehat{p} \operatorname{sen}(\phi)=e^{-i \phi}(\widehat{p}+i \widehat{q})$.

E da mesma maneira obtemos:

$$
\begin{aligned}
\mathfrak{R}(\phi, \widehat{n})(\widehat{p}-i \widehat{q}) & =e^{i \phi}(\widehat{p}-i \widehat{q}), \\
\mathfrak{R}(\phi, \widehat{n}) \widehat{n} & =\widehat{n},
\end{aligned}
$$

Portanto, os autovalores da rotação $\mathfrak{R}(\phi, \widehat{n})$ são $1, e^{i \phi}$ e $e^{-i \phi}$. 
Pelo ponto de vista geométrico a rotação $\mathfrak{R}=\mathfrak{R}(\phi, \widehat{n})$ é uma transformação linear de vetores com duas propriedades:

$$
\begin{gathered}
\mathfrak{R}:<\vec{r}, \vec{s}>\quad \rightarrow \quad<\mathfrak{R} \vec{r}, \mathfrak{R} \vec{s}>=<\vec{r}, \vec{s}>, \\
\mathfrak{R}: \vec{r} \times \vec{s} \quad \rightarrow \quad \mathfrak{R} \vec{r} \times \mathfrak{R} \vec{s}=\mathfrak{R}(\vec{r} \times \vec{s}) .
\end{gathered}
$$

Reciprocamente, qualquer transformação linear no espaço Euclideano que satisfaça essas propriedades é uma rotação.

Introduzimos rotações como simetrias euclideanas. É necessario observar que duas rotações tendo um centro em comum, combinadas, formam uma outra rotação, isto é, as rotações formam um grupo. O "produto"de duas rotações $\mathfrak{R}_{1}$ e $\mathfrak{R}_{2}$, denotado por $\mathfrak{R}_{1} \mathfrak{R}_{2}$ resulta numa transformação que é associativa:

$$
\left(\mathfrak{R}_{1} \mathfrak{R}_{2}\right) \mathfrak{R}_{3}=\mathfrak{R}_{1}\left(\mathfrak{R}_{2} \mathfrak{R}_{3}\right)
$$

O inverso de $\mathfrak{R}(\phi, \widehat{n})$ é $\mathfrak{R}(-\phi, \widehat{n})$ também denotado por $\mathfrak{R}^{-1}(\phi, \widehat{n})$. A rotação identidade é a rotação nula $(\phi=0)$, isto é, sem rotação.

Rotações possuindo um eixo em comum formam um subgrupo abeliano:

$$
\mathfrak{R}\left(\phi_{1}, \widehat{n}\right) \mathfrak{R}\left(\phi_{2}, \widehat{n}\right)=\mathfrak{R}\left(\phi_{2}, \widehat{n}\right) \mathfrak{R}\left(\phi_{1}, \widehat{n}\right)=\mathfrak{R}\left(\phi_{1}+\phi_{2}, \widehat{n}\right)
$$

Em geral, rotações não comutan.

Rotações possuindo ângulo em comum obedecem a relação classe de ângulo [9],

$$
\mathfrak{L} \mathfrak{R}(\phi, \widehat{n}) \mathfrak{L}^{-1}=\mathfrak{R}(\phi, \mathfrak{L} \widehat{n}),
$$

onde $\mathfrak{L}$ é uma rotação que leva o eixo $\widehat{n}$ em um novo eixo de rotação: $\widehat{n}^{\prime}=L \widehat{n}$.

O ponto de vista geométrico descrito acima focaliza a atenção em pontos como entidades e as transformações em que eles se submetem. Um procedimento menos intuitivo, porém, mais poderoso é a abordagem analítica, a qual foca atenção em três medidas correlacionadas a qualquer ponto. Nessa abordagem uma tripla é uma relação entre duas estruturas: o ponto designado pela tripla e do referencial pelo qual os números sao definidos. Especificamente o referencial será denotado por $F=\left(\widehat{e}_{1}, \widehat{e}_{2}, \widehat{e}_{3}\right)$, consistindo de vetores $\widehat{e}_{i}, i=1,2,3$, que possuem uma origem comum, comprimento unitário $\left(\widehat{e}_{i} \cdot \widehat{e}_{i}=1\right)$, são mutuamente ortogonais, $\left(\widehat{e}_{i} \cdot \widehat{e}_{j}=0, i \neq j\right)$ e são orientados $\widehat{e}_{1}=\widehat{e}_{2} \times \widehat{e}_{3}$. Usando o referêncial $F$, a tripla $x=\left(x_{1}, x_{2}, x_{3}\right)$ associado ao ponto $x$ satisfaz $x_{i}=<\vec{x}, \widehat{e}_{i}>$, onde $\vec{x}$ é o vetor da origem ao ponto $x$. 
Uma rotação $\mathfrak{R}$ tem uma bem definida ação em cada um dos vetores que definem um referencial. Uma matriz $\mathbf{R}, 3 \times 3$, pode ser associada a essa rotação $\mathfrak{R}$ pela seguinte associação:

$$
\mathbf{R}_{i j}=<\widehat{e}_{i}, \mathfrak{R} \widehat{e}_{j}>
$$

que é o elemento pertencente a linha $i$ e coluna $j$ de $\mathbf{R}$. E cada rotação $\mathfrak{R}$ corresponde a uma matriz $\mathbf{R}$.

A ação de $\mathfrak{R}$ num vetor arbitrário $\vec{x}=\sum x_{i} \widehat{e}_{i}$, pode agora ser escrito como $\mathfrak{R} \vec{x}=$ $\sum_{i}\left(\sum_{j} \mathbf{R}_{i j} x_{j} \widehat{e}_{i}\right)$. Escrevendo os vetores $\vec{x}$ e $\mathcal{R} \vec{x}=\vec{x}^{\prime}$ como matrizes colunas , $x=$ $\operatorname{col}\left(x_{1}, x_{2}, x_{3}\right)$ e $x^{\prime}=\operatorname{col}\left(x_{1}^{\prime}, x_{2}^{\prime}, x_{3}^{\prime}\right)$, obtemos a equação matricial $x^{\prime}=\mathbf{R} x$. Desta maneira obtemos a correspondência $\mathfrak{R} \rightarrow \mathbf{R}$ entre rotações e matrizes de tal maneira que o produto de duas rotações $\mathfrak{R}_{1} \mathfrak{R}_{2}$ corresponde ao produto matricial $\mathbf{R}_{1} \mathbf{R}_{2}$. A correspondência $\mathfrak{R} \rightarrow \mathbf{R}$ é uma representação do grupo das matrizes.

Reformulando rotações nesses termos obtemos o resultado matricial:

$$
\mathfrak{R}(\phi, \widehat{n}) \rightarrow \mathbf{R}(\phi, \widehat{n})=\mathbb{1}_{3}+\mathbf{N} \operatorname{sen}(\phi)+\mathbf{N}^{2}(1-\cos (\phi)) .
$$

Onde $\mathbb{1}_{3}$ é a matriz identidade $3 \times 3$ e $\mathbf{N}$ é uma matriz antisimetrica determinada pelo eixo $\widehat{n}$ da rotação $\mathbf{N}_{i j}=-\epsilon_{i j k} n_{k}$, onde $\epsilon_{i j k}=+1$ e -1 , respectivamente, para permutações pares e impares de $(i, j, k)$ de $(1,2,3)$, e 0 de outra maneira.

Esse resultado para a matriz $\mathbf{R}(\phi, \widehat{n})$ mostra que o conjunto das matrizes $3 \times 3$ representando as rotações são reais, ortogonais $\left(\mathbf{R}^{t}=R^{-1}\right)$ e própria $(\operatorname{det} \mathbf{R}=1)$. Denotamos esse grupo de matrizes por $S O(3)$, ou seja,

$$
S O(3)=\left\{R \in M_{R}(3) ; \quad R R^{t}=R^{t} R=I, \quad \operatorname{det}(R)=1 .\right\}
$$

Enfatizamos que a representação de uma rotação $\mathcal{R}$ por uma matriz $3 \times 3$, real, própria e ortogonal é definida relativamente a fixado referencial, $F=\left(\widehat{e}_{1}, \widehat{e}_{2}, \widehat{e}_{3}\right)$.

Agora apresentaremos uma definição, segundo Cartan, do que é chamado de spin, ou spinors, dependendo da literatura onde se encontram.

Spinors são introduzidos na mecânica quântica para descrever particulas que possuem momento angular' meio-inteiro ("spin"). Na matemática, contudo, spinors foram descobertos antes ${ }^{2}$. Cartan definiu spinors em sua forma mais geral desde 1913.

\footnotetext{
${ }^{1}$ Momento angular será apresentado mais adiante, sem causar problemas no entendimento no decorrer do texto.

${ }^{2}$ Spinors são atualmente implicitos nos quaternios Hamiltonianos e nas transformações homograficas de Klein e Cayley, mas fazer esse conceito explicito não é trivial. Para definir spinors a partir dos quaternios é equivalente a definir a função onda a partir da matriz densidade, ou, algebricamente, determinar os ideais proprios maximais de uma algebra.
} 
A vantagem da abordagem de Cartan é puramente geométrica e por causa dessa origem geométrica as matrizes de Pauli, conhecidas na mecância quantica, aparecem por vontade própria sem qualquer consideração quântica.

A chave da abordagem de Cartan é considerar um vetor isotrópico (um vetor de comprimento nulo) no espaço (complexo) tri-dimensional Euclideano. As componetes de tal vetor satisfazem $x_{1}^{2}+x_{2}^{2}+x_{3}^{2}=0$ e podemos associar a esse vetor dois números complexos $\xi_{0}, \xi_{1}$ dados por:

$$
x_{1}=\xi_{0}^{2}-\xi_{1}^{2}, \quad x_{2}=i\left(\xi_{0}^{2}+\xi_{1}^{2}\right), \quad x_{3}=-2 \xi_{0} \xi_{1} .
$$

Alternativamente, podemos definir $\xi_{0}$ e $\xi_{1}$ em termos do vetor isotropico $x$ por:

$$
\xi_{0}= \pm \sqrt{\frac{1}{2}\left(x_{1}-i x_{2}\right)}, \quad \xi_{1}= \pm \sqrt{-\frac{1}{2}\left(x_{1}+i x_{2}\right)} .
$$

Cartan observou que não é possível atribuir um sinal exato às equações acima, pois sob a rotação de $2 \pi, \xi_{0}$ e $\xi_{1}$ invertem o sinal mas o vetor isotrópico retorna a sua posição original. Esse comportamento é que define que um spinor volta a sua identidade apenas depois de uma rotação de $4 \pi$. Um spinor é um tensor Euclideano.

Vamos determinar como um spinor se transforma sob rotação, usando as propriedades conhecidas de um vetor sob rotação. O spinor $\left(\xi_{0}, \xi_{1}\right)$ está associado com o vetor isotrópico $\left(x_{1}, x_{2}, x_{3}\right)$, sob rotação $\mathbf{R}$ temos $x \rightarrow x^{\prime}=\mathbf{R} x$ e como, comprimentos são preservados, o vetor isotrópico $x^{\prime}$ determina o spinor transformado $\left(\xi_{0}^{\prime}, \xi_{1}^{\prime}\right)$. Portanto, para $\left(\xi_{0}^{\prime}\right)^{2}$ encontramos da equação 1.1 .2 que:

$$
\begin{gathered}
\left(\xi_{0}^{\prime}\right)^{2}=\frac{1}{2}\left(\left(R_{11}-i R_{21}\right) x_{1}+\left(R_{12}-i R_{22}\right) x_{2}+\left(R_{13}-i R_{23}\right) x_{3}\right) \\
=\frac{1}{2}\left(R_{11}-i R_{21}+i R_{12}+R_{22}\right) \xi_{0}^{2}-\left(R_{13}-i R_{23}\right) \xi_{0} \xi_{1}+\frac{1}{2}\left(-R_{11}+i R_{21}+i R_{12}+R_{22}\right) \xi_{1}^{2} .
\end{gathered}
$$

Vale observar que o lado direito dessa igualdade é um quadrado perfeito, pois o descriminante se anula em consequência da ortogonalidade da matriz R. A expressão para $\left(\xi_{1}^{\prime}\right)^{2}$ é também um quadrado perfeito. Portanto, ambos $\xi_{0}^{\prime}$ e $\xi_{1}^{\prime}$ são determinados linearmente em termos de $\xi_{0}$ e $\xi_{1}$ a menos de sinal \pm . O sinal relativo de $\xi_{0}^{\prime}$ e $\xi_{1}^{\prime}$ não é ambiguo, mas determinado pelo sinal de $x_{3}^{\prime}$.

A forma explícita de uma transformação linear induzida por um spinor pela rotação do vetor isotrópico correspondente $x$ será desenvolvida na próxima subseção. Mostraremos que uma rotação $x \rightarrow x^{\prime}=\mathbf{R} x$ de um vetor isotrópico induz a rotação $\xi^{\prime}=U \xi$ de spinors, onde $\xi=\left(\xi_{0}, \xi_{1}\right)^{t}$ e $U$ é uma matriz unitária unimodular obtida pelos elementos $R_{i j}$ de $\mathbf{R}$. 
As equações (1.1.2) para o vetor isotropico $x$ associado com o spinor $\xi$ verifica a razão $\xi_{0} / \xi_{1}$ na forma $\xi_{0} / \xi_{1}=-\left(x_{1}-i x_{2}\right) / x_{3}=x_{3} /\left(x_{1}+i x_{2}\right)$. A relação expressa por essa razão também pode ser escrita em notação matricial

$$
(x \cdot \sigma) \xi=0
$$

onde $x \cdot \sigma$ denota $x_{1} \sigma_{1}+x_{2} \sigma_{2}+x_{3} \sigma_{3}$ e $\sigma_{i}(i=1,2,3)$ denota as matrizes de Pauli:

$$
\sigma_{1}=\left(\begin{array}{ll}
0 & 1 \\
1 & 0
\end{array}\right), \sigma_{2}=\left(\begin{array}{cc}
0 & -i \\
i & 0
\end{array}\right), \sigma_{3}=\left(\begin{array}{cc}
1 & 0 \\
0 & -1
\end{array}\right) .
$$

Portanto, $x \cdot \sigma$ é a matriz $2 \times 2$ dada por:

$$
x \cdot \sigma=\left(\begin{array}{cc}
x_{3} & x_{1}-i x_{2} \\
x_{1}+i x_{2} & -x_{3}
\end{array}\right) .
$$

A matriz acima, assim como as matrizes de Pauli, surge naturalmente na aplicação de Cartan, dando o vetor isotrópico $x$ associado ao spinor $\xi$.

Cartan também notou que a matriz acima fornece uma maneira geral de associar uma matriz $2 \times 2$ complexa a um vetor arbitrario, e reciprocamente,

$$
x=\left(x_{1}, x_{2}, x_{3}\right) \leftrightarrow\left(\begin{array}{cc}
x_{3} & x_{1}-i x_{2} \\
x_{1}+i x_{2} & -x_{3}
\end{array}\right)
$$

Será essa associação que usaremos para desenvolver a relação entre as rotações $(S U(2))$ e as matrizes ortogonais próprias reais.

\subsubsection{Relação entre $S U(2)$ e $S O(3)$.}

Observamos agora que para $x$ real a matriz $x \cdot \sigma$ é hermitiana e de traço nulo. E essas proprieades são preservadas por transformações unitárias:

$$
x \cdot \sigma \rightarrow x^{\prime} \cdot \sigma=U(x \cdot \sigma) U^{\dagger},
$$

onde $U \cdot U^{\dagger}=U^{\dagger} \cdot U=\sigma_{0}=I d_{2}$ e $U^{\dagger}$ é a transposta conjugada de $U$.

Portanto, correspondendo a transformação unitaria para spinors, $\xi^{\prime}=U \xi$, temos a transformação linear de vetores $x^{\prime}=\mathbf{R} x$ determinada pela equação acima:

$$
x_{i}^{\prime}=\frac{1}{2} \operatorname{Tr}\left(\sigma_{i} U(x \cdot \sigma) U^{\dagger}\right)=\sum_{j} R_{i j} x_{j},
$$




$$
R_{i j}=\frac{1}{2} \operatorname{Tr}\left(\sigma_{i} U \sigma_{j} U^{\dagger}\right)
$$

onde $\operatorname{Tr}$ denota o traço da matriz.

Uma expressão alternativa e útil para a matriz $\mathbf{R}=\left(R_{i j}\right)$ pode ser obtida reescrevendo a transformação (1.1.3) primeiro como $\eta^{\prime}=(U \otimes \bar{U}) \eta$, onde $\eta=\operatorname{col}\left(x_{3}, x_{1}-\right.$ $\left.i x_{2}, x_{1}+i x_{2},-x_{3}\right)$ e depois como uma relação entre as matrizes colunas $\operatorname{col}\left(x_{1}^{\prime}, x_{2}^{\prime}, x_{3}^{\prime}, \xi^{\prime}\right)$ e $\operatorname{col}\left(x_{1}, x_{2}, x_{3}, \xi\right)$, onde $\xi$ é uma variável qualquer. O resultado é:

$$
\left(\begin{array}{cc}
\mathbf{R} & 0 \\
0 & 1
\end{array}\right)=S^{\dagger}(U \otimes \bar{U}) S,
$$

onde $U \otimes \bar{U}$ denota o produto direto de matrizes de $U$ por $\bar{U}$ e $S$ é a matriz unitaria unimodular dada por

$$
S=\frac{1}{\sqrt{2}}\left(\begin{array}{cccc}
0 & 0 & 1 & -i \\
1 & -i & 0 & 0 \\
1 & i & 0 & 0 \\
0 & 0 & -1 & -i
\end{array}\right)
$$

Seja $U(2)$ denotando o grupo das matrizes $2 \times 2$ das matrizes unitárias:

$$
U(2)=\left\{U: U U^{\dagger}=U^{\dagger} U=\mathbb{1}_{2}\right\}
$$

Usando as equações (1.1.5) e (1.1.6) acima podemos provar que:

(a) $R$ é real, própria e ortogonal para cada $U \in U(2)$;

(b) Se $U$ é levada em $R$ pela relação (1.1.5), então $U^{\prime} U \rightarrow R^{\prime} R$;

(c) o conjunto de elementos de $U(2)$, os quais são levados à identidade de $S O(3)$ (núcleo da aplicação) pela relação (1.1.5), é $\left\{e^{i \phi} \sigma_{0}: 0 \leq \phi \leq 2 \pi\right\}$;

(d) existe um elemento $U \in U(2)$, tal que $U \rightarrow R$, para cada $R \in S O(3)$, a aplicação é sobrejetora.

Para aplicações é útil termos uma forma explicita determinando o conjunto das matrizes $U \in U(2)$ soluções da equação (1.1.5) que corresponde a especificar $R \in$ $S O(3)$. Para isso, observamos que cada $U \in U(2)$ deve ser escrita na forma $U=e^{i \phi} U_{0}$, $0 \leq \phi<2 \pi$, onde $U_{0}$ pertence ao grupo das matrizes $2 \times 2$ unitárias unimodulares:

$$
S U(2)=\left\{U_{0}: U_{0} U_{0}^{\dagger}=\mathbb{1}_{2}, \operatorname{det} U_{0}=1\right\}
$$

Em seguida usamos o fato que $U_{0} \in S U(2)$ tem a forma: 


$$
U_{0}=U\left(\alpha_{0}, \alpha\right)=\left(\begin{array}{cc}
\alpha_{0}-i \alpha_{3} & -i \alpha_{1}-\alpha_{2} \\
-i \alpha_{1}+\alpha_{2} & \alpha_{0}+i \alpha_{3}
\end{array}\right)=\alpha_{0} \mathbb{1}_{2}-i \alpha \cdot \sigma
$$

onde $\left(\alpha_{0}, \alpha\right)=\left(\alpha_{0}, \alpha_{1}, \alpha_{2}, \alpha_{3}\right)$ são os parâmetros reais de Rodriges-Euler, os quais definem a superficie da esfera unitária $S^{3}$, isto é, $\alpha_{0}^{2}+\alpha_{1}^{2}+\alpha_{2}^{2}+\alpha_{3}^{2}=1$. O elemento $R=R\left(\alpha_{0}, \alpha\right) \in S O(3)$ correspondente a $U_{0}$ é encontrado a partir de (1.1.6):

$$
R=\left(\begin{array}{ccc}
\alpha_{0}^{2}+\alpha_{1}^{2}-\alpha_{2}^{2}-\alpha_{3}^{2} & 2 \alpha_{1} \alpha_{2}-2 \alpha_{0} \alpha_{3} & 2 \alpha_{1} \alpha_{3}+2 \alpha_{0} \alpha_{2} \\
2 \alpha_{1} \alpha_{2}+2 \alpha_{0} \alpha_{3} & \alpha_{0}^{2}+\alpha_{2}^{2}-\alpha_{3}^{2}-\alpha_{1}^{2} & 2 \alpha_{2} \alpha_{3}-2 \alpha_{0} \alpha_{1} \\
2 \alpha_{1} \alpha_{3}-2 \alpha_{0} \alpha_{2} & 2 \alpha_{2} \alpha_{3}+2 \alpha_{0} \alpha_{1} & \alpha_{0}^{2}+\alpha_{3}^{2}-\alpha_{1}^{2}-\alpha_{2}^{2}
\end{array}\right)
$$

Uma solução de (1.1.10) para $\left(\alpha_{0}, \alpha\right)$ em termos de elementos da matriz $R_{i j}$ de $R$ é encontrado facilmente:

$$
\begin{gathered}
\operatorname{tr} R \neq-1: \quad \alpha_{0} \equiv \frac{1}{2}(1+\operatorname{tr} R)^{\frac{1}{2}}, \quad \alpha_{i} \equiv-\left(R_{j k}-R_{k j}\right) / 4 \alpha_{0}, \quad i, j, k \quad \text { cyclic }, \\
\operatorname{tr} R=-1: \quad \alpha_{0}=0, \quad \alpha_{i}=\left(\operatorname{sign}_{i}\right)\left[\left(1+R_{i i}\right) / 2\right]^{\frac{1}{2}} \\
\operatorname{sign}_{1}=1, \quad \operatorname{sign}_{2}=\operatorname{sign}_{12}, \quad \operatorname{sign}_{3}=\operatorname{sign}_{13} .
\end{gathered}
$$

Resumindo, temos apenas duas matrizes unimodulares unitárias que resolvem a equação (1.1.5) para cada $\mathbf{R}$ específico, a saber, $U_{0}$ dada pela equação (1.1.9) com os parametros descritos acima, e $-U_{0}$, da mesma matriz: $\left\{U_{0},-U_{0}\right\} \rightarrow \mathbf{R}$. O conjunto de todas as matrizes $U \in U(2)$ soluções de (1.1.5) é portanto, $\left\{e^{i \phi} U_{0}: 0 \leq \phi<2 \pi\right\}$.

Escrevendo as matrizes unimodulares unitárias $2 \times 2$ na forma (1.1.9) mostra claramente que os elementos de $S U(2)$ estão em correspondência injetora com os pontos de $S^{3}$, os quais definem a esfera unitária no $\mathbb{R}^{4}$. Como $\left(\alpha_{0}, \alpha\right)$ percorrem todos os pontos de $S^{3}$, as matrizes (1.1.9) e (1.1.10) enumeram, respectivamente, todos os elementos de $S U(2)$ e todos os elementos de $S O(3)$, sendo evidente de (1.1.10) que $\left(\alpha_{0}, \alpha\right) \in S^{3}$ e seu antipoda $\left(-\alpha_{0},-\alpha\right) \in S^{3}$ define a mesma matriz ortogonal propria $\mathbf{R}$.

\subsubsection{Parametrizações do grupo de rotações}

A representação das rotações por matrizes $3 \times 3$ reais, ortogonais e próprias e das matrizes $2 \times 2$ unitárias unimodulares pode ser implementada por várias parame- 
trizações de suma importância nas aplicações físicas. Em adição aos parâmetros de Euler-Rodrigues $\left(\alpha_{0}, \alpha\right)$, introduzidos na subseção acima, existem três parametrizações 3 comuns das rotações encontrados na literatura. Vamos discutir cada uma dessas parametrizações brevemente e fazer as referências na literatura para quem vier a se interessar por um conhecimento mais profundo.

(a) Os parâmetros $(\phi, \widehat{n})$, os quais Euler introduziu para caracterização de direção e ângulo de rotação: usamos esses parâmetros no inicio da seção, ao definir a ação de uma rotação em um vetor. A matriz associada $\mathbf{R}(\phi, \widehat{n})$ é dada pela equação (1.1.1). Uma forma exponencial pode também ser obtida dessa matriz notando que $\mathbf{N}^{3}=-\mathbf{N}$ :

$$
\mathbf{R}(\phi, \widehat{n})=e^{\phi N}, \quad 0 \leq \phi \leq \pi, \quad<\widehat{n}, \widehat{n}>=1 .
$$

O domínio $0 \leq \phi \leq \pi,<\widehat{n}, \widehat{n}>=1$ cobre o grupo de elementos de $S O(3)$ exatamente uma vez, exceto que, para $\phi=\pi, \widehat{n} \mathrm{e}-\widehat{n}$ determina a mesma rotação. Os elementos de $S O(3)$ estão portanto em uma correspondência injetora com os pontos da esfera sólida de raio $\pi$, onde pontos opostos diametralmente na superficie são identificados.

A relação inversa, expressando $\phi$ e $\widehat{n}$ em termos de $R_{i j}$ de uma matriz real, própria e ortogonal $\mathbf{R}$, pode ser encontrada diretamente resolvendo a equação (1.1.1) para $\phi$ e N. Os resultados serão:

$$
\begin{gathered}
\cos \phi=\frac{(-1+\operatorname{tr} R)}{2} \\
n_{i} \operatorname{sen} \phi=-\left(R_{j k}-R_{k j}\right), \quad i, j, k \quad \text { cclicos. }
\end{gathered}
$$

Como cada matriz real, própria e ortogonal $R$ tem $-1 \leq \operatorname{tr} R \leq 3$ a relação (1.1.13) determina um único ângulo no intervalo $0 \leq \phi \leq \pi$. Com o $\phi$ determinado, encontramos $n_{i}$ da relação (1.1.14), a menos que $\phi=\pi$. Para $\phi=\pi$, temos duas soluções, $\pm \widehat{n}$, para a direção, onde $\widehat{n}=\alpha$ é obtida da equação (1.1.11).

O conjunto das matrizes unitárias unimodulares na parametrização $(\phi, \widehat{n})$ é obtida pelas equações (1.1.9),(1.1.11),(1.1.13) e (1.1.14):

$$
U=U(\psi, \widehat{n})=\sigma_{0} \cos (\psi / 2)-i(\widehat{n} \cdot \sigma) \operatorname{sen}(\psi / 2)=e^{-i \psi(\widehat{n} \cdot \sigma) / 2},
$$

aqui a última igualdade é provada por expansão direta, usando $(\widehat{n} \cdot \sigma)^{2}=\sigma_{0}$. O domínio de definição do parâmetro $(\psi, \widehat{n})$ é agora $<\widehat{n}, \widehat{n}\rangle=1$ e $0 \leq \psi \leq 2 \pi$, de tal maneira

\footnotetext{
${ }^{3}$ Existe uma quarta parametrização, a parametrização de parametros racionais, Cayley [5], que é de importância técnica mas não há muito uso prático na aplicação de momento angular.
} 
que o conjunto

$$
\left\{\left(\alpha_{0}, \alpha\right)=(\cos (\psi / 2), \widehat{n} \operatorname{sen}(\psi / 2))\right\}
$$

cobre toda a esfera $S^{3}$ exatamente uma vez.

Dividindo os pontos na esfera unitária $S^{3}$ em dois hemisférios abertos:

$$
\begin{gathered}
\left\{\left(\cos \left(\frac{\phi}{2}\right), \widehat{n} \operatorname{sen}\left(\frac{\phi}{2}\right)\right): \quad 0 \leq \phi<\pi, \quad<\widehat{n}, \widehat{n}>=1\right\}, \\
\left\{\left(\cos \left(\frac{2 \pi-\phi}{2}\right), \widehat{n} \operatorname{sen}\left(\frac{2 \pi-\phi}{2}\right)\right): \quad 0 \leq \phi<\pi, \quad<\widehat{n}, \widehat{n}>=1\right\} .
\end{gathered}
$$

O conjunto de pontos $\{(0, \widehat{n}):<\widehat{n}, \widehat{n}>=1\}$ correspondente a $\phi=\pi$ (esfera unitária $\left.S^{2}\right)$ constitue a fronteira comum aos dois hemisférios. O par de matrizes unitárias unimodulares, $U(\phi, \widehat{n})$ e $U(2 \pi-\phi,-\widehat{n})=-U(\phi, \widehat{n})$ correspondente ao ponto no primeiro hemisfério e seu ponto diametralmente oposto no segundo hemisfério, mapeado via equação (1.1.5) em $\mathbf{R}(\phi, \widehat{n})$.

(b) Os parâmetros de Cayley-Klein, projeção estereográfica da esfera $S^{2}$ no plano complexo conduz a uma relação entre rotações e matrizes unitárias que é equivalente a aquela que obtemos usando os spinors de Cartan. Rotações de pontos na esfera correspondem a transformações homográficas do plano, também chamadas de tranformações lineares fracionais ou transformações bilineares. Essas relações foram descobertas por Klein [15] e desenvolvidas por Klein [16] e Cayley [11].

Uma correspondência entre um ponto $\left(x_{1}, x_{2}, x_{3}\right)$ na esfera $x_{1}^{2}+x_{2}^{2}+x_{3}^{2}=1$ e um ponto $(\xi, \eta)$ do plano equatorial é estabelecido pela projeção estereográfica a partir do polo norte $n_{0}=(0,0,1)$. Assim a reta que passa pelo polo norte $n_{0}$ e qualquer ponto $\left(x_{1}, x_{2}, x_{3}\right) \in S^{2}$, com exceção do polo $n_{0}$, determina um ponto $(\xi, \eta)$ no plano equatorial, reciprocamente, a reta que passa por qualquer ponto finito $(\xi, \eta)$ no plano equatorial e o polo norte $n_{0}$ determina um ponto $\left(x_{1}, x_{2}, x_{3}\right)$ na esfera.

Considerações geométricas entre triângulos semelhantes produzem as relações $\xi=$ $x_{1} /\left(1-x_{3}\right)$ e $\eta=x_{2} /\left(1-x_{3}\right)$, isto é:

$$
\zeta=\xi+i \eta=\frac{x_{1}+i x_{2}}{1-x_{3}}=\frac{1+x_{3}}{x_{1}-i x_{2}} .
$$

Vamos expor agora o principal teorema das transformações homográficas (para demonstração seguir referências de [5], págs 21 e 22).

Seja $x \rightarrow x^{\prime}=\mathbf{R} x$ uma transformação ortogonal própria na esfera. Então o $\xi \eta$-plano submetido a tranformação determinada pela transformação homográfica de 
$\zeta=\xi+i \eta$

$$
\zeta \rightarrow \zeta^{\prime}=\frac{\overline{u_{11}} \zeta+\overline{u_{12}}}{\overline{u_{21}} \zeta+\overline{u_{22}}}
$$

onde $u_{i j}$ são elementos da matriz unitária $U=\left(u_{i j}\right)$, a qual está relacionada com $\mathbf{R}=\left(R_{i j}\right)$ por

$$
R_{i j}=\frac{1}{2} \operatorname{tr}\left(\sigma_{i} U \sigma_{j} U^{\dagger}\right)
$$

Observações:

(a) Observe que as matrizes unitárias $U$ e $\lambda U,(|\lambda|=1)$, determinam a mesma transformação homográfica no plano.Podemos então escolher $U$ unimodular, cujo caso as duas matrizes unimodulares $U$ e $-U$ determinam a mesma transformação.

(b) As transformações ortognais próprias da esfera a as transformações de $\xi \eta$-plano são injetoras. É a identificação das matrizes unitárias unimodulares $2 \times 2 \mathrm{com}$ as transformações homográficas que então verificam as relações "dois pra um"com essas transformações, portanto, com as matrizes ortogonais próprias.

(c) A transformação homográfica (1.1.15) $\zeta \rightarrow \zeta^{\prime}$ do plano complexo correspondente a rotação $x \rightarrow x^{\prime}=R x$ da esfera foi obtida pela primeira vez por Cayley [11] na forma

$$
\zeta^{\prime}=\frac{\left(\alpha_{0}+i \alpha_{3}\right) \zeta+\left(i \alpha_{1}-\alpha_{2}\right)}{\left(i \alpha_{1}+\alpha_{2}\right) \zeta+\left(\alpha_{0}-i \alpha_{3}\right)}
$$

onde $\alpha_{i}=n_{i} \operatorname{sen}(\psi / 2), \alpha_{0}=\cos (\psi / 2),<\widehat{n}, \widehat{n}>=1,0 \leq \psi \leq 2 \pi$. Os quatro números complexos $(a, b, c, d)=\left(\alpha_{0}+i \alpha_{3}, i \alpha_{1}-\alpha_{2}, i \alpha_{1}+\alpha_{2}, \alpha_{0}-i \alpha_{3}\right)$ ou ligeiras variantes destes, são agora chamados de Cayley-Klein parâmetros. Enquanto que os quatro números reais $\left(\alpha_{0}, \alpha\right)$ definindo um ponto na superficie da esfera unitária no espaço 4-dimensional, $S^{3}$ são conhecidos como parâmetros de Euler-Rodrigues.

(c) Os ângulos de Euler, os três ângulos introduzidos por Euler para caracterização das rotações são talvez os mais amplamente usados nas aplicações físicas. Isso pode explicar a existência das muitas variações em suas definições na literatura.

Geometricamente, os ângulos de Euler $(\alpha \beta \gamma)$ são descritos pela seqüência de três rotações de vetores, os quais descreveremos em termos de um referencial fixo $\left(\widehat{e}_{1}, \widehat{e}_{2}, \widehat{e}_{3}\right)$ :

$R_{1}$ : rotação de ângulo $\alpha$ em torno de $\widehat{n}_{1}=(0,0,1)$,

$R_{2}$ : rotação de ângulo $\beta$ em torno de $\widehat{n}_{2}=(-\operatorname{sen}(\alpha), \cos (\alpha), 0)$,

$R_{3}$ : rotação de ângulo $\gamma$ em torno de $\widehat{n}_{3}=(\cos (\alpha) \operatorname{sen}(\beta), \operatorname{sen}(\alpha) \operatorname{sen}(\beta), \cos (\beta))$.

Outra maneira de descrever os ângulos de Euler em termos de outras rotações é a seguinte: 


$$
R(\alpha \beta \gamma)=R_{3} R_{2} R_{1}=R_{1}^{\prime} R_{2}^{\prime} R_{3}^{\prime},
$$

$R_{3}^{\prime}$ : rotação de $\gamma$ em torno de $\widehat{e}_{3}=(0,0,1)$,

$R_{2}^{\prime}$ : rotação de $\beta$ em torno de $\widehat{e}_{2}=(0,1,0)$,

$R_{1}^{\prime}$ : rotação de $\alpha$ em torno de $\widehat{e}_{3}=(0,0,1)$.

Se considerarmos essas rotações como transformações atuantes, então $\mathbf{R}(\alpha \beta \gamma)$ mapea um vetor arbitrário $\vec{x}$ em um novo vetor $\vec{x}^{\prime}=\mathbf{R}(\alpha \beta \gamma) \vec{x}$. As componetes de $\vec{x}^{\prime}$ estão relacionadas com as coordenadas de $\vec{x}$, no referencial fixado, por

$$
\begin{aligned}
& x^{\prime}=\mathbf{R}(\alpha \beta \gamma) x, \\
& \mathbf{R}(\alpha \beta \gamma)=\left(\begin{array}{ccc}
\cos (\alpha) & -\operatorname{sen}(\alpha) & 0 \\
\operatorname{sen}(\alpha) & \cos (\alpha) & 0 \\
0 & 0 & 1
\end{array}\right)\left(\begin{array}{ccc}
\cos (\beta) & 0 & \operatorname{sen}(\beta) \\
0 & 1 & 0 \\
-\operatorname{sen}(\beta) & 0 & \cos (\beta)
\end{array}\right)\left(\begin{array}{ccc}
\cos (\gamma) & -\operatorname{sen}(\gamma) & 0 \\
\operatorname{sen}(\gamma) & \cos (\gamma) & 0 \\
0 & 0 & 1
\end{array}\right) \\
& =\left(\begin{array}{ccc}
\cos (\alpha) \cos (\beta) \cos (\gamma)-\sin (\alpha) \sin (\gamma) & -\cos (\alpha) \cos (\beta) \operatorname{sen}(\gamma)-\operatorname{sen}(\alpha) \cos (\gamma) & \cos (\alpha) \operatorname{sen}(\beta) \\
\operatorname{sen}(\alpha) \cos (\beta) \cos (\gamma)+\cos (\alpha) \operatorname{sen}(\gamma) & -\operatorname{sen}(\alpha) \cos (\beta) \operatorname{sen}(\gamma)+\cos (\alpha) \cos (\gamma) & \operatorname{sen}(\alpha) \operatorname{sen}(\beta) \\
-\operatorname{sen}(\beta) \cos (\gamma) & \operatorname{sen}(\beta) \operatorname{sen}(\gamma) & \cos (\beta)
\end{array}\right)
\end{aligned}
$$

O domínio de definição dos ângulos de Euler na equação acima é

$$
0 \leq \alpha<2 \pi, \quad 0 \leq \beta \leq \pi, \quad 0 \leq \gamma<2 \pi
$$

Conjuntos distintos de números $(\alpha \beta \gamma)$ que estão nesses intervalos correspondem a diferentes rotações exceto para $\beta=0$ ou $\beta=\pi$. Se $\beta=0$, a rotação é de ângulo $\alpha+\gamma$ em torno de $(0,0,1)$; se $\beta=\pi$, a rotação é de angulo $\alpha-\gamma$ em torno de $(0,0,1)$. Nesses casos valores distintos de $\alpha$ e $\gamma$ podem determinar a mesma rotação.

A inversa de $\mathbf{R}(\alpha \beta \gamma)$ é

$$
\mathbf{R}^{-1}(\alpha \beta \gamma)=\mathbf{R}(2 \pi-\gamma, \pi-\beta, 2 \pi-\alpha)
$$

A parametrização de $U \in S U(2)$ usando os ângulos de Euler é dada por:

$$
\begin{gathered}
U(\alpha \beta \gamma)=e^{-i \alpha \sigma_{3} / 2} e^{-i \beta \sigma_{2} / 2} e^{-i \gamma \sigma_{3} / 2} \\
=\left(\begin{array}{cc}
e^{-i \alpha / 2} \cos (\beta / 2) e^{-i \gamma / 2} & -e^{-i \alpha / 2} \operatorname{sen}(\beta / 2) e^{i \gamma / 2} \\
e^{i \alpha / 2} \operatorname{sen}(\beta / 2) e^{-i \gamma / 2} & e^{i \alpha / 2} \cos (\beta / 2) e^{i \gamma / 2}
\end{array}\right),
\end{gathered}
$$


onde o domínio da definição de $(\alpha \beta \gamma)$ é agora estendido a

$$
0 \leq \alpha<2 \pi, \quad 0 \leq \beta \leq \pi \quad \text { ou } \quad 2 \pi \leq \beta \leq 3 \pi, \quad 0 \leq \gamma<2 \pi
$$

Notando que $U(\alpha, \beta+2 \pi, \gamma)=-U(\alpha \beta \gamma)$ vemos que ambos $U(\alpha \beta \gamma)$ e $U(\alpha, \beta+2 \pi, \gamma)$, $0 \leq \beta \pi$ mapeam o mesmo $\mathbf{R}(\alpha \beta \gamma)$.

Os itens (a), (b) e (c) foram retirados de [5].

\subsection{Momento angular e harmônicos esféricos}

Um fato notável é que nos primeiros papers estabelecendo a forma matricial da mecânica quântica, os operadores para momento angular foram introduzidos e as consequências decisivas do momento angular quantizado deduzidas. As técnicas desenvolvidas para esse tratamento são espantosamente próximas, mas independentes, a aquelas desenvolvidas por Cartan em seu tratamento definitivo de grupos de Lie (semisimples) no qual o momento angular $S U(2)$ é o protótipo.

Na mecânica clássica, o momento angular, $\vec{L}$, de uma partícula é definido como o produto vetorial do vetor posição $r=(x, y, z)$ (relativo a uma origem fixa) pelo vetor momento $p=\left(p_{x}, p_{y}, p z\right)$,

$$
L=r \times p,
$$

ou em componentes,

$$
\begin{aligned}
& L_{x}=y p_{z}-z p_{y}, \\
& L_{y}=z p_{x}-x p_{z}, \\
& L_{z}=x p_{y}-y p_{x} .
\end{aligned}
$$

É natural estender essa definição para a mecânica quântica interpretando os símbolos como operadores, isto é, redefinimos o momento angular de uma partícula a ser um operador, $\widehat{L}$, onde as componentes do operador posição $r$ e do operador momento $p$ obedecem as regras de comutatividade, $\left[p_{i}, x_{j}\right]=-i \hbar \delta_{i j}$, em termos de componentes, $\widehat{p}=(\hbar / i)\left(\frac{\partial}{\partial x}, \frac{\partial}{\partial y}, \frac{\partial}{\partial z}\right)=(\hbar / i) \nabla$ na expressão clássica de $L$. Assim obtemos o operador vetor de momento angular,

$$
\widehat{L}=r \times \widehat{p}=\frac{i}{\hbar} r \times\left(\frac{\partial}{\partial x}, \frac{\partial}{\partial y}, \frac{\partial}{\partial z}\right),
$$

ou em componentes, 


$$
\begin{aligned}
& \widehat{L}_{x}=\frac{i}{\hbar}\left(y \frac{\partial}{\partial z}-z \frac{\partial}{\partial y}\right), \\
& \widehat{L}_{y}=\frac{i}{\hbar}\left(z \frac{\partial}{\partial x}-x \frac{\partial}{\partial z}\right), \\
& \widehat{L}_{z}=\frac{i}{\hbar}\left(x \frac{\partial}{\partial y}-y \frac{\partial}{\partial x}\right) .
\end{aligned}
$$

Enquanto que as componentes do momento comutam uma com a outra, isto é, $\left[\widehat{p}_{x}, \widehat{p}_{y}\right]=\widehat{p}_{x} \widehat{p}_{y}-\widehat{p}_{y} \widehat{p}_{x}=0$, e para os outros também, as componentes do momento angular já não satisfazem a isso. Pois,

$$
\begin{aligned}
& {\left[\widehat{L}_{x}, \widehat{L}_{y}\right]=i \hbar \widehat{L}_{z},} \\
& {\left[\widehat{L}_{y}, \widehat{L}_{z}\right]=i \hbar \widehat{L}_{x},} \\
& {\left[\widehat{L}_{z}, \widehat{L}_{x}\right]=i \hbar \widehat{L}_{y} .}
\end{aligned}
$$

Como os comutadores não se anulam, uma autofunção de $\widehat{L}_{z}$ não pode, em geral, ser também uma autofunção de $\widehat{L}_{y}$. Se ainda, além da equação de autovalor $\widehat{L}_{z} Y=l_{z} Y$, a relação $\widehat{L}_{y} Y=l_{y} Y$ também se verificar teremos uma contradição para relação do comutador $\left[\widehat{L}_{y}, \widehat{L}_{z}\right]=i \hbar \widehat{L}_{x}$ avaliada em $Y$ :

$$
\left(\widehat{L}_{y} \widehat{L}_{z}-\widehat{L}_{z} \widehat{L}_{y}\right) Y=\left(l_{y} l_{z}-l_{z} l_{y}\right) Y \neq i \hbar \widehat{L}_{x} Y
$$

Essa observação é equivalente a afirmação que operadores não comutativos não têm autofunções simultâneas, exceto para os casos triviais.

Contudo, existe um outro operador, o quadrado do operador vetorial do momento angular, $\widehat{L}^{2}=\widehat{L}_{x}^{2}+\widehat{L}_{y}^{2}+\widehat{L}_{z}^{2}$, o qual comuta com qualquer componente $\left[\widehat{L}^{2}, \widehat{L}_{a}^{2}\right]=0$, para $a=x, y, z$. Essa relação é facilmente verificada com a ajuda das propriedades do comutador, por exemplo,

$$
\begin{gathered}
{\left[\widehat{L}_{x}^{2}+\widehat{L}_{y}^{2}+\widehat{L}_{z}^{2}, \widehat{L}_{z}\right]=\left[\widehat{L}_{x}^{2}, \widehat{L}_{z}\right]+\left[\widehat{L}_{y}^{2}, \widehat{L}_{z}\right]} \\
=\widehat{L}_{x}\left[\widehat{L}_{x}, \widehat{L}_{z}\right]+\left[\widehat{L}_{x}, \widehat{L}_{z}\right] \widehat{L}_{x}+\widehat{L}_{y}\left[\widehat{L}_{y}, \widehat{L}_{z}\right]+\left[\widehat{L}_{y}, \widehat{L}_{z}\right] \widehat{L}_{y} \\
=\widehat{L}_{x}\left(-i h \widehat{L}_{y}\right)-i h \widehat{L}_{y} \widehat{L}_{x}+\widehat{L}_{y}(i h) \widehat{L}_{x}+i h \widehat{L}_{x} \widehat{L}_{y}=0 .
\end{gathered}
$$

Portanto simultâneas autofunções para $\widehat{L}^{2}$ e qualquer das componentes, por exemplo, $\widehat{L}_{z}$ pode ser encontrado. Para a discussão seguinte é mais conveniente usarmos a notação em coordenadas polares $r, \vartheta$ e $\phi$ do que as coordenadas cartesianas $x, y$ e $z$. 
No sistema de coordenadas polares um ponto é dado por sua distância $r$ da origem, seu angulo polar $\vartheta$ e seu azimute $\phi$. As relações entre as coordenadas cartesianas e polares são:

$$
\begin{gathered}
x=r \operatorname{sen} \vartheta \cos \phi \\
y=r \operatorname{sen} \vartheta \operatorname{sen} \phi \\
z=r \cos \vartheta
\end{gathered}
$$

Os operadores de momento angular ficam:

$$
\begin{gathered}
\widehat{L}_{x}=i h\left(\operatorname{sen} \phi \frac{\partial}{\partial \vartheta}+\cot \vartheta \cos \phi \frac{\partial}{\partial \phi}\right) \\
\widehat{L}_{y}=-i h\left(\cos \phi \frac{\partial}{\partial \vartheta}-\cot \vartheta \sin \phi \frac{\partial}{\partial \phi}\right) \\
\widehat{L}_{y}=-i h \frac{\partial}{\partial \phi}, \\
\widehat{L}^{2}=-\hbar^{2}\left[\frac{1}{\operatorname{sen} \vartheta} \frac{\partial}{\partial \vartheta}\left(\operatorname{sen} \vartheta \frac{\partial}{\partial \vartheta}\right)+\frac{1}{\operatorname{sen}^{2} \vartheta} \frac{\partial^{2}}{\partial \phi^{2}}\right] .
\end{gathered}
$$

Observação: para se chegar a essas expressões (coord. polares) se faz necessário o uso das seguintes condições: $d x\left(\frac{\partial}{\partial l}\right)=\delta_{x, l}$.

Podemos escrever equações de autovalores para os dois operadores $\widehat{L}^{2}$ e $\widehat{L}_{z}$ :

$$
\begin{gathered}
\widehat{L}^{2} Y_{l}^{m}=l(l+1) \hbar^{2} Y_{l}^{m}, \\
\widehat{L}_{z} Y_{l}^{m}=m \hbar Y_{l}^{m} .
\end{gathered}
$$

Ambos os operadores possuem como autofunções os harmônicos esféricos $Y_{l}^{m}(\vartheta, \phi)$. Os autovalores do momento angular ao quadrado são $l(l+1) h^{2}$. Esse número quantico do momento angular $l$ podem tomar apenas valores $l=0,1,2 \ldots$. Portanto, em contraste a mecânica classica, o momento angular ao quadrado só pode tomar valores discretos que são inteiros múltiplos de $\hbar^{2}$. Correspondentemente, os autovalores do componente $z, \widehat{L}_{z}$, do momento angular são $m \hbar$. O número quântico $m$ pode variar entre $-l \leq m \leq l$. Por fatos históricos o número quântico $m$ é as vezes chamado de número quantico magnético.

Os harmônicos esféricos $Y_{l}^{m}(\vartheta, \phi)$ possuem uma representação explícita a qual é comumente baseada nos polinômios de Legendre, 


$$
P_{l}(u)=\frac{1}{2^{l} l !} \frac{d^{l}}{d u^{l}}\left[\left(u^{2}-1\right)^{l}\right] .
$$

Os polinômios de Legendre são um caso especial das funções associadas de Legendre $P_{l}^{m}$, as quais são definidas por

$$
P_{l}^{m}(u)=\left(1-u^{2}\right)^{m / 2} \frac{d^{m}}{d u^{m}} P_{l}(u)
$$

onde $m=0,1,2 \ldots, l$.

Colocando $P_{0}^{0}=1, P_{l}^{m}=0$ para $l<m$ e

$$
P_{l}^{l}(z)=(-1)^{l}(2 l-1) ! !\left(1-z^{2}\right)^{l / 2}
$$

os polinomiais $P_{l}^{m}$ também podem ser obtidos a partir da fórmula recorrente:

$$
(l-m) P_{l}^{m}(z)=(2 l-1) z P_{l-1}^{m}(z)-(l+m-1) P_{l-2}^{m}(z) .
$$

Alguns exemplos de polinômios de Legendre:

$$
\begin{gathered}
P_{1}^{0}(z)=z, \quad P_{1}^{1}(z)=-\left(1-z^{2}\right)^{1 / 2}, \quad P_{2}^{0}=\frac{1}{2}\left(3 z^{2}-1\right), \\
P_{2}^{1}(z)=-3 z\left(1-z^{2}\right)^{1 / 2}, \quad P_{2}^{2}(z)=3\left(1-z^{2}\right), \quad P_{3}^{0}(z)=\frac{1}{2} z\left(5 z^{2}-3\right), \\
P_{3}^{1}(z)=\frac{3}{2}\left(1-5 z^{2}\right)\left(1-z^{2}\right)^{1 / 2}, \quad P_{3}^{2}(z)=15 z\left(1-z^{2}\right), \quad P_{3}^{3}(z)=-15\left(1-z^{2}\right)^{3 / 2} .
\end{gathered}
$$

Finalmente, para $m \geq 0$, os harmônicos esféricos $Y_{l}^{m}$ possuem a seguinte representação:

$$
Y_{l}^{m}(\vartheta, \phi)=(-1)^{m} \sqrt{\frac{2 l+1}{4 \pi} \cdot \frac{(l-m) !}{(l+m) !}} P_{l}^{m}(\cos \vartheta) e^{i m \phi} .
$$

Para valores negativos de $m, m=-1,-2, \ldots,-l$, os harmônicos esféricos são,

$$
Y_{l}^{-m}(\vartheta, \phi)=(-1)^{m} \overline{Y_{l}^{m}}(\vartheta, \phi) .
$$

Apesar dos polinômios de Legendre $P_{l}(u)$ e as funções associadas de Legendre $P_{l}^{m}$ serem funções reais no argumento $u$, os harmônicos esféricos $Y_{l}^{m}$ são funções complexas 
em seus argumentos. As definições e as plotagens ${ }^{4}$ implicam $\left|Y_{l}^{m}\right|^{2}$ depende somente de $\vartheta$, pois exceto de um fato de normalização, este é igual a $\left|P_{l}^{m}(\cos \vartheta)\right|^{2}$. Alguns harmônicos esféricos:

$$
\begin{gathered}
Y_{0}^{0}(\vartheta, \phi)=\frac{1}{2} \frac{1}{\sqrt{\pi}} ; \\
Y_{1}^{-1}(\vartheta, \phi)=\frac{1}{2} \sqrt{\frac{1}{2 \pi}} \operatorname{sen} \vartheta e^{-i \phi} ; \\
Y_{1}^{0}(\vartheta, \phi)=\frac{1}{2} \sqrt{\frac{3}{\pi}} \cos \vartheta ; \\
Y_{1}^{1}(\vartheta, \phi)=\frac{1}{2} \sqrt{\frac{1}{2 \pi}} \operatorname{sen} \vartheta e^{i \phi} .
\end{gathered}
$$

Como as variáveis dos harmônicos esféricos são o ângulo polar $\vartheta$ e o azimute $\phi$ do sistema de coordenadas esféricos é vantajoso representar $\left|Y_{l}^{m}\right|^{2}$ em tal sistema de coordenadas. De fato, $\left|Y_{l}^{m}(\vartheta, \phi)\right|^{2}$ é o comprimento do arco entre os ângulos $\vartheta$ e $\phi$, da origem a superfície. Neste caso, $\left|Y_{0}^{0}\right|^{2}=\frac{1}{4 \pi}$ vem a ser a esfera. Já para todos os possíveis valores de $l$ e $m$ as funções $\left|Y_{l}^{m}\right|^{2}$ são rotacionalmente simétricos ao eixo $z$. Podem se anular para alguns valores de $\vartheta$. Esses valores são chamados de $\vartheta$ nós se ocorrem para valores de $\vartheta$ além do zero e de $\pi$. Nota-se que $\left|Y_{l}^{l}\right|^{2}$ não possui nós, enquanto que $\left|Y_{l}^{m}\right|^{2}$ possui $l-|m|$ nós.

Podemos escrever os harmônicos esféricos em função das coordenadas cartesianas também, então por exemplo, para $l=1,2,3$, obteremos:

$$
\begin{gathered}
Y_{1}^{1}=-\sqrt{\frac{3}{2}}(x+i y), \quad Y_{1}^{0}=\sqrt{3} z, \quad Y_{1}^{-1}=\sqrt{\frac{3}{2}}(x-i y) \\
Y_{2}^{2}=-\sqrt{\frac{15}{8}}(x+i y)^{2}, \quad Y_{2}^{1}=-\sqrt{\frac{15}{8}}(x+i y) z, \quad Y_{2}^{0}=-\frac{\sqrt{5}}{2}\left(3 z^{2}-1\right) \\
Y_{3}^{3}=-\frac{\sqrt{35}}{4}(x+i y)^{3}, \quad Y_{3}^{2}=\frac{3}{2} \sqrt{\frac{35}{6}}(x+i y)^{2} z, \\
Y_{3}^{1}=-\frac{\sqrt{21}}{4}(x+i y)\left(5 z^{2}-1\right), \quad Y_{3}^{0}=-\frac{\sqrt{7}}{2}\left(5 z^{3}-3 z\right) .
\end{gathered}
$$

\footnotetext{
${ }^{4}$ olhar [8],pgs 184 e 185.
} 
Os polinômios de Legendre possuem a seguinte propriedade de ortogonalidade:

$$
\int_{-1}^{1} P_{l}(u) P_{l^{\prime}}(u) d u=\frac{2}{2 l+1} \delta_{l l^{\prime}}
$$

Aqui $\delta_{l l^{\prime}}$ é o símbolo de Kronecker.

Para os harmônicos esféricos a relação de ortonormalidade verifica

$$
\int_{\cos \vartheta=0}^{-1} \int_{\phi=0}^{2 \pi} \overline{Y_{l}^{m}}(\vartheta, \phi) Y_{l^{\prime}}^{m^{\prime}}(\vartheta, \phi) d \cos \vartheta d \phi=\delta_{l l^{\prime}} \delta_{m m^{\prime}} .
$$

Como a integral é estendida sobre todos os possiveis ângulos $\vartheta$ e $\phi$ podemos dizer que a integração é realizada sobre um sólido cheio $\Omega=4 \pi$ e podemos escrever a integral acima abreviadamente por:

$$
\int \overline{Y_{l}^{m}}(\vartheta, \phi) Y_{l^{\prime}}^{m^{\prime}}(\vartheta, \phi) d \Omega=\delta_{l l^{\prime}} \delta_{m m^{\prime}}
$$

Vimos que os harmônicos esféricos são autofunções para os operadores $\widehat{L}^{2}$ e $\widehat{L}_{z}^{2}$. Aplicações para os operadores $\widehat{L}_{x}$ e $\widehat{L}_{y}$ verificam:

$$
\begin{aligned}
& \widehat{L}_{x} Y_{l}^{m}=\frac{\hbar}{2} \sqrt{l(l+1)-m(m+1)} Y_{l}^{m+1}-\frac{\hbar}{2} \sqrt{l(l+1)-m(m-1)} Y_{l}^{m-1}, \\
& \widehat{L}_{y} Y_{l}^{m}=\frac{\hbar}{2 i} \sqrt{l(l+1)-m(m+1)} Y_{l}^{m+1}-\frac{\hbar}{2 i} \sqrt{l(l+1)-m(m-1)} Y_{l}^{m-1},
\end{aligned}
$$

mostrando que $Y_{l}^{m}$ não são autofunções de $\widehat{L}_{x}$ e $\widehat{L}_{y}$.

Usando as relações de ortonormalidade dos harmônicos esféricos calculamos os valores esperados ${ }^{1}$ das três componentes e do quadrado do momento angular,

$$
\begin{aligned}
& <L_{x}>_{l m}=\int\left(Y_{l}^{m}\right)^{*}(\vartheta, \phi) \widehat{L}_{x} Y_{l}^{m}(\vartheta, \phi) d \Omega=0, \\
& <L_{y}>_{l m}=\int\left(Y_{l}^{m}\right)^{*}(\vartheta, \phi) \widehat{L}_{y} Y_{l}^{m}(\vartheta, \phi) d \Omega=0, \\
& <L_{z}>_{l m}=\int\left(Y_{l}^{m}\right)^{*}(\vartheta, \phi) \widehat{L}_{z} Y_{l}^{m}(\vartheta, \phi) d \Omega=m \hbar,
\end{aligned}
$$

\footnotetext{
${ }^{1} \mathrm{O}$ valor expectativo ou valor médio da posição de uma particula descrita pela função de onda $\psi(x, t)$ é dado por $\langle x\rangle=\int_{-\infty}^{+\infty} \psi^{*}(x, t) x \psi(x, t) d x$. Mais detalhes ver [8].
} 


$$
<\mathbf{L}^{2}>=\int\left(Y_{l}^{m}\right)^{*}(\vartheta, \phi) \widehat{\mathbf{L}}^{2} Y_{l}^{m}(\vartheta, \phi) d \Omega=l(l+1) \hbar^{2} .
$$

Os valores esperados para as três componentes $(0,0, m)$ não podem ser interpretados como três componentes de um vetor, pois o módulo desse vetor ao quadrado é $m^{2}$ o qual é sempre menor do que o valor esperados $l(l+1) \hbar^{2}$ de $\widehat{\mathbf{L}}^{2}$,

$$
<L_{x}>_{l m}^{2}+<L_{y}>_{l m}^{2}+<L_{z}>_{l m}^{2}=m^{2} \hbar^{2} \leq l(l+1) \hbar^{2} .
$$

A razão para esse resultado vem do fato de calcularmos o valor expectativo do quadrado de cada componente do momento angular. Como $Y_{l}^{m}$ é uma autofunção de $\widehat{L}_{z}$ encontramos

$$
<L_{z}^{2}>=\int\left(Y_{l}^{m}\right)^{*}(\vartheta, \phi) \widehat{L}_{z}^{2} Y_{l}^{m}(\vartheta, \phi) d \Omega=\hbar^{2} m^{2}
$$

Para as duas outras componentes faremos uso das equações para $\widehat{L}_{x} Y_{l}^{m}$ e $\widehat{L}_{y} Y_{l}^{m}$ dados acima, e obtemos:

$$
<L_{x, y}^{2}>=\int\left(Y_{l}^{m}\right)^{*}(\vartheta, \phi) \widehat{L}_{x, y}^{2} Y_{l}^{m}(\vartheta, \phi) d \Omega=\frac{\hbar^{2}}{2}\left(l(l+1)-m^{2}\right)
$$

O não anulamento desses dois valores expectativos $<L_{x, y}^{2}>$ resolve a diferença acima:

$$
\begin{gathered}
<L_{x}^{2}>_{l m}+<L_{y}^{2}>_{l m}+<L_{z}^{2}>_{l m} \\
=\frac{\hbar^{2}}{2}\left(l(l+1)-m^{2}\right)+\frac{\hbar^{2}}{2}\left(l(l+1)-m^{2}\right)+\hbar^{2} m^{2}=l(l+1) .
\end{gathered}
$$

Com a ajuda dos resultados para os valores esperados dos quadrados das componentes calcula-se a variância ${ }^{2}$ das componentes do momento angular:

$$
\left(\operatorname{var}\left(L_{z}\right)\right)_{l m}=0, \quad\left(\operatorname{var}\left(L_{x}\right)\right)_{l m}=\frac{\hbar^{2}}{2}\left[l(l+1)-m^{2}\right]=\left(\operatorname{var}\left(L_{y}\right)\right)_{l m} .
$$

As incertezas ${ }^{3}$ das três componentes do momento angular para as autofunções vem a ser

$$
\left(\Delta L_{x}\right)_{l m}=\left(\Delta L_{y}\right)_{l m}=\hbar \frac{1}{\sqrt{2}}\left[l(l+1)-m^{2}\right]^{1 / 2}, \quad\left(\Delta L_{z}\right)_{l m}=0 .
$$

\footnotetext{
${ }^{2}$ A variância da posição é o valor esperado do quadrado da diferença entre a posição e sua expectativa: $\operatorname{var}(x)=<(x-<x>)^{2}>$. [8].

${ }^{3} \mathrm{O}$ raiz da variância da posição $\Delta x=\sqrt{\operatorname{var}(x)}$, determina a largura do comprimento de onda na posição da variavel $x$ e portanto é uma medida de incerteza sobre onde a particula está localizada.
} 
Isto mostra que as autofunções $Y_{l l}$ pertencentes ao autovalor $l=m$ satisfazem uma regra particular ao longo do conjunto $-l \leq m \leq l$ :

(i) Para $Y_{l l}(\vartheta, \varphi)$ o valor da componente $z, m=l$, é próxima ao valor expectativo do módulo $\sqrt{l(l+1)}$.

(ii) As incertezas dos componentes do momento angular sao pequenas.

Por essas razões toma-se as autofunções $Y_{l l}$ como o estado quântico mais próximo ao vetor clássico,

$$
L=<L_{z}>_{l l} e_{z}=\hbar l e_{z}
$$

do momento angular. Aqui $e_{z}$ é um vetor de comprimento unitário na direção de $z$.

As autofunções do momento angular estão completamente especificadas pelos autovalores $l(l+1) \hbar^{2}$ do quadrado $\widehat{L}^{2}$ do operador momento angular $\widehat{L}=\left(L_{x}, L_{y}, L_{z}\right)$ e pelos autovalores $m \hbar$ de $\widehat{L}_{z}$, a componente $z$ de $\widehat{L}$. Para distinguir essa coordenada das outras, indica-se a direção de $z$ por $e_{z}=(0,0,1)$ e trocando a notação dos harmônicos esféricos

$$
Y_{l m}(\vartheta, \varphi) \rightarrow Y_{l m}\left(\vartheta, \varphi, e_{z}\right)
$$

Escolhe-se outra direção denotada pelo vetor unitário $n=\left(n_{x}, n_{y}, n_{z}\right)$ como a direção $z$ em outro sistema de coordenadas. Nessa estrutura o angulo polar é denotado por $\vartheta^{\prime}$ e o azimute $\varphi^{\prime}$. As autofunções de $\widehat{L}^{2}$ e $\widehat{L}_{z}^{\prime}=n \cdot \widehat{L}=n_{x} \widehat{L}_{x}+n_{y} \widehat{L}_{y}+n_{z} \widehat{L}_{z}$ são então $Y_{l m}\left(\vartheta^{\prime}, \varphi^{\prime}, n\right)$.Denotaremos o ângulo polar e o ângulo azimutal da direção $n$ no sistema original de coordenadas por $\theta$ e $\phi$,

$$
n=(\operatorname{sen}(\theta) \cos (\phi), \operatorname{sen}(\theta) \operatorname{sen}(\phi), \cos (\theta)) .
$$

Encontramos que as autofunções $Y_{l l}(\vartheta, \varphi, n)$ é o estado quântico que mais se aproxima do vetor momento angular classico $L=<\widehat{L}_{z}^{\prime}>_{l l} n=\hbar l n$. Agora analizemos a função de onda $Y_{l m}\left(\vartheta, \varphi, e_{z}\right)$ do momento angular total $l \hbar$ e $z$ componente $m \hbar$ pela função de onda $Y_{l l}(\vartheta, \varphi, n)$. No nosso caso análise de amplitude é:

$$
a=N \int_{0}^{2 \pi} \int_{-1}^{1} Y_{l l}^{*}(\vartheta, \varphi, n) Y_{l m}\left(\vartheta, \varphi, e_{z}\right) d \cos \vartheta d \varphi=N D_{m l}^{(l)}(\phi, \theta, 0)
$$

Essas funções e a notação $D_{m l}^{(l)}$ foram introduzidas por Eugene P. Wigner e são conhecidas na literatura como funções de Wigner. A constante de normalização $N$ será determinada por uma escolha apropriada de normalização,

$$
|N|^{2}=\frac{(2 l+1)(l+1)}{4 \pi l}, \quad \text { para } \quad l=1,2,3 \ldots .
$$


Consideremos o quadrado absoluto da análise de aplitude,

$$
|a|^{2}=f_{l m}(\theta, \phi)=|N|^{2}\left|D_{m l}^{(l)}(\phi, \theta, 0)\right|^{2}=|N|^{2}\left[d_{m l}^{(l)}(\theta)\right]^{2} .
$$

Onde $d_{m l}^{(l)}(\theta)$ é também referida como função de Wigner na literatura. E possue representação explicita,

$$
d_{m l}^{(l)}(\theta)=\sqrt{\frac{(2 l) !}{(l+m) !(l-m) !}} \cos (\theta / 2)^{l+m} \operatorname{sen}(\theta / 2)^{l-m} .
$$

\subsection{Os multiplets do momento angular}

Vamos lidar agora com a formulação padrão da mecânica quântica, isto é, um espaço de Hilbert sobre os números complexos com observáveis representados por operadores lineares Hermitianos. Denotamos os vetores no espaço de Hilbert por "kets" $\mid \phi>$ e os vetores do estaço dual por bras,$<\psi \mid$. O produto interno é denotado pelo "bracket":

$$
<\psi \mid \phi>\in \mathbb{C} \text {. }
$$

Procuramos deduzir consequências a partir das relações de comutatividade de $\mathbf{J}$, o operador momento angular que é um observável tal que $\mathbf{J} \times \mathbf{J}=\mathbf{i} \mathbf{J}$

A estratégia escolhida por Cartan [10] e por Born et al. [7] é converter o problema num problema de autovalor de matriz finito dimensional. Para fazer isso vamos primeiro obervar que o operador $\mathbf{J}^{2}$,

$$
\mathbf{J}^{2} \equiv J_{1}^{2}+J_{2}^{2}+J_{3}^{2}
$$

comuta com $\mathbf{J}$ :

$$
\left[\mathbf{J}^{2}, J_{i}\right]=0
$$

O problema de autovalor é então encontrar o conjunto de auto-kets ${ }^{5}$ tal que $\mathbf{J}^{2}$ e $J_{3}$ são diagonais:

$$
\begin{aligned}
& \mathbf{J}^{2}\left|J^{2^{\prime}}, J_{3}{ }^{\prime}>=J^{2^{\prime}}\right| J^{2^{\prime}}, J_{3}{ }^{\prime}>, \\
& J_{3}\left|{J^{2}}^{\prime}, J_{3}{ }^{\prime}>=J^{3^{\prime}}\right| J^{2^{\prime}}, J_{3}{ }^{\prime}>,
\end{aligned}
$$

\footnotetext{
$5 ", \quad$, denota "autovalor", ou seja, $\mathbf{J}^{2} \rightarrow J^{2 \prime} \in \mathbb{R}$
} 
onde $J^{2}$ é um número real não negativo e $J_{3}{ }^{\prime}$ é um número real. Essas propriedades seguem do fato de $J_{i}$ serem operadores Hermitianos.

Vamos definir os operadores não Hermitianos (extensão complexa da algebra de Lie):

$$
J_{+} \equiv J_{1} \pm i J_{2}, \quad J_{+}^{\dagger}=J_{-},
$$

onde $\dagger$ denota conjugação Hermitiana. Esses novos operadores satisfazem as regras:

$$
\left[\mathbf{J}^{2}, J_{ \pm}\right]=0, \quad\left[J_{3}, J_{ \pm}\right]= \pm J_{ \pm}, \quad\left[J_{+}, J_{-}\right]=2 J_{3},
$$

os quais são conseqûencias imediatas de $\mathbf{J} \times \mathbf{J}=\mathbf{i} \mathbf{J}$

Assumimos que existe um auto-ket que satisfaz $J_{3}\left|J^{2^{\prime}}, J_{3}{ }^{\prime}>=J^{3^{\prime}}\right| J^{2^{\prime}}, J_{3}{ }^{\prime}>$ e formamos novos kets $J_{ \pm}\left|J^{2^{\prime}}, J_{3}{ }^{\prime}>\equiv\right| \pm>$. Usando as regras de comutatividade, encontramos que o ket $\mid+>$, se não nulo, possue os autovalores

$$
\begin{gathered}
\mathbf{J}^{2}\left|+>=J^{2^{\prime}}\right|+>, \\
J_{3}\left|+>=\left(J_{3}{ }^{\prime}+1\right)\right|+>.
\end{gathered}
$$

Similarmente, $\mid->$, se não nulo, possui os autovalores $J^{2 \prime}$ e $J_{3}{ }^{\prime}-1$. É justamente esse comportamento que conta para os nomes "aising"para o operador $J_{+}$e "lowering"para o operador $L_{-}$.

Esse processo pode ser repetido e aparentemente, gera autokets todos pertencentes a $J^{2^{\prime}}$, mas para os autovalores de $J_{3}$, serão $J_{3}{ }^{\prime}+k$, onde $k$ é um inteiro.

Um método padrão para enumerar os autovalores é introduzir $j=(k+l) / 2$ e seja $m$ um número qualquer dentro do conjunto $\{j, j-1, \cdots,-j\}$. A notação $\mid j m>$ é também introduzida para designar uma normalização simultânea do auto ket de $\mathbf{J}^{2}$ e $J_{3}$. Assim teremos

$$
\begin{gathered}
\mathbf{J}^{2}|j m>=j(j+1)| j m>, \\
J_{3}|j m>=m| j m>,
\end{gathered}
$$

onde $j$ pertence ao conjunto $\{0,1 / 2,1,3 / 2,2, \ldots\}$ e para dado $j$, o valor de $m$ varia entre $j$ e $-j$ em passos de unidade.

Observe que o único auto ket $\mid J^{2}{ }^{\prime}, J_{3}{ }^{\prime}>$ do qual o conjunto de auto kets $\{\mid j m>$ : $m=j, j-1, \cdots,-j\}$ foi gerado é denotado pela nova notação por $\mid j, j-k>$. Contudo, podemos agora obter o conjunto a partir de $\mid j j>$. As propriedades definidas de $\mid j j>\neq 0$ são:

$$
\mathbf{J}^{2}|j j>=j(j+1)| j j>, \quad J_{3}|j j>=j| j j>,
$$




$$
\begin{gathered}
J_{+}\left|j j>=0, \quad J^{2 j+1}\right| j j>=0, \\
J_{-}^{j-m} \mid j j>\neq 0, \quad m=j, j-1, \ldots,-j .
\end{gathered}
$$

Os auto kets normalizados $\mid j m>$, para $\mid j j>$ normalizado, são dados por:

$$
\left|j m>=\left[\frac{(j+m) !}{(2 j) !(j-m) !}\right]^{1 / 2} J_{-}^{j-m}\right| j j>
$$

onde o fator normalização é obtido usando a identidade:

$$
J_{+}^{k} J_{-}^{k}=\sum_{s=1}^{k}\left[\mathbf{J}^{2}-\left(J_{3}-s\right)\left(J_{3}-s+1\right)\right] .
$$

Com isso, $J_{+}$e $J_{-}$avaliados nos auto kets $\mid j m>$, são:

$$
\begin{aligned}
& J_{+}\left|j m>=[(j-m)(j+m+1)]^{1 / 2}\right| j m+1>, \\
& J_{-}\left|j m>=[(j+m)(j-m+1)]^{1 / 2}\right| j m-1>.
\end{aligned}
$$

Finalmente, observamos que os auto kets correspondentes a distintos valores de $j$ e distintos valores de $m$ são, em consequência das propriedades Hermitianas de $J_{i}$, ortogonais:

$$
<j^{\prime} m^{\prime} \mid j m>=\delta_{j^{\prime} j} \delta_{m^{\prime} m}
$$

Resumimos que em um espaço de Hilbert para o qual os operadores $J_{i}$ são Hermitianos, os únicos autovalores de $\mathbf{J}^{2}$ são da forma $j(j+1)$, onde $j \in\{0,1 / 2,1,3 / 2,2, \cdots\}$. Para cada $j$ existem $2 j+1$ auto kets ortonormais de $J_{3}$ possuindo autovalores $m=$ $j, j-1, \cdots,-j$.

É importante observar que quando apresentamos um certo espaço de Hilbert sobre o qual está definido um conjunto de operadores Hermitianos $J_{1}, J_{2}, J_{3}$, satisfazendo $\left[J_{i}, J_{j}\right]=i \varepsilon_{i j k} J_{k}$, a teoria desenvolvida acima não faz nenhuma previsão sobre quais valores de $j \in\{0,1 / 2,1,3 / 2,2, \cdots\}$ irá ocorrer e nem quais serão suas multiplicidades.

Apesar da simplicidade de se obter esses resultados, eles são de fundamental importância. E todo o futuro desenvolvimento é consequência destes.

Algumas observações (para mais detalhes ver [6]):

a) O autovalor $j$ é chamado de momento angular. O autovalor $m$ é chamado de z-componente do momento angular, ou às vezes, como número quântico projeção. E no uso matemático, o autovalor $m$ é chamado como "weight." 
b) Determinando $j$ à partir da equação quadrática $J_{2}{ }^{\prime}=j(j+1)$, leva a uma segunda solução: $-j-1$. Esse "momento angular negativo"define uma reflexão simétrica importante para o "coupling"de coeficientes na teoria de momento angular.

c) Os autovalores $\{(j, m)\}$ se dividem em dois conjuntos: inteiros e meio inteiros. O momento angular orbital se refere ao movimento espacial de partículas, tem a forma $\vec{L}=\vec{r} \times \vec{p}$ e sempre possui momento angular inteiro. Momento angular spin se refere a uma propriedade intrínseca da partícula e pode ser integral ou meio integral (meio inteiro ou ímpar).

\subsection{Coeficientes de Wigner e $3 \mathrm{j}$ simbolo.}

Antes de apresentar os símbolos de Wigner e de Racah, precisamos antes introduzir o conceito de adição de momento angular.

Nas seções anteriores determinamos todos os possíveis autoespaços $H_{j}$ (base padrão de vetores kets $\mid j m>$ ) correspondendo ao maximal observavel do momento angular $\left[\mathbf{J}^{2} \rightarrow j(j+1), J_{3} \rightarrow m\right]$. Como notado no começo podemos considerar esses estados como uma descrição do momento angular total de um sistema composto ou, analogamente, como o estado válido para descrever o momento angular de uma única particula. Essa liberdade de interpretação existe pois, a independencia da cinemática implica que $\mathbf{J}=\sum_{i=1}^{n} \mathbf{J}(i)$ obedece as mesmas relações de comutatividade de $\mathbf{J}(i)$ separadamente e conceitualmente, o monento angular total do sistema existe por causa da invariância da descrição do sistema composto por rotações.

É natural agora examinar essas duas interpretações simultaneamente e considerar o problema de adição de momento angular de duas partículas independentes (ou sistemas). A física especifica as condições necessárias, que são as seguintes:

a) O momento angular de duas particulas são simultaneamente observáveis; portanto, satisfazem a relação de comutatividade:

$$
\left[J_{i}(1), J_{k}(2)\right]=0 \quad i, k=1,2,3 .
$$

Correspondentemente, os vetores kets do sistema composto são produtos de vetores ket das particulas individuais.

b) As duas particulas pertence ao autoespaço momento angular $H_{j_{i}}(i),(\mathrm{i}=1,2)$ cujas bases são dadas por

$$
\left\{\mid j_{i} m_{i}>: j_{i} \text { fixo, }-j_{i} \leq m_{i} \leq j_{i}\right\}
$$


c) O momento angular total é a soma $\mathbf{J}=\mathbf{J}(\mathbf{1})+\mathbf{J}(\mathbf{2})$. Como $\mathbf{J}(\mathbf{k}) \times \mathbf{J}(\mathbf{k})=i \mathbf{J}(\mathbf{k})$ e as componentes de $\mathbf{J}(\mathbf{1})$ comutam com os elementos de $\mathbf{J}(\mathbf{2})$, vemos que

$$
\mathbf{J} \times \mathbf{J} \equiv i \mathbf{J}
$$

Essas condições implicam que os vetores kets nos quais o operador momento angular total atua é o espaço produto tensorial $H_{j_{1}}(1) \otimes H_{j_{2}}(2)$ gerado pelo conjunto ortonormal produto de vetores bases:

$$
\left\{\left|j_{1} m_{1}>\right| j_{2} m_{2}>=\left|j_{1} m_{1}>\otimes\right| j_{2} m_{2}>:-j_{1} \leq m_{1} \leq j_{1},-j_{2} \leq m_{2} \leq j_{2}\right\}
$$

A ação do operador momento angular total $J_{i}=J_{i}(1)+J_{i}(2)$ sobre o espaço $H_{j_{1}}(1) \otimes$ $H_{j_{2}}(2)$ é definida por

$$
J_{i}\left|j_{1} m_{1}>\right| j_{2} m_{2}>=\left(J_{i}(1) \mid j_{1} m_{1}>\right)\left|j_{2} m_{2}>+\right| j_{1} m_{1}>\left(J_{i}(2) \mid j_{2} m_{2}>\right) .
$$

Mais explicitamente, essa ação leva a matriz $\left(2 j_{1}+1\right)\left(2 j_{2}+1\right)$-dimensional, $M_{i}$, do momento angular total $J_{i}$ dado por

$$
J_{i} \rightarrow M_{i}=J^{\left(j_{1}\right)} \otimes \mathbb{1}^{\left(j_{2}\right)}+\mathbb{1}^{\left(j_{1}\right)} \otimes J^{\left(j_{2}\right)},
$$

onde $\mathbb{1}^{(j)}$ denota a matriz unitária de ordem $(2 j+1)$ e $\otimes$ designa o produto direto de matrizes.

Vemos que, pela escolha da base para o espaço produto tensorial $H_{j_{1}}(1) \otimes H_{j_{2}}(2)$, os autovalores de $J_{3}$ são $m=m_{1}+m_{2}$. Os possiveis valores para $m$ para $-j_{1} \leq m_{1} \leq j_{1}$ e $-j_{2} \leq m_{2} \leq j_{2}$ são determinados pela lista de pontos $\left(m_{1}, m_{2}\right), m=m_{1}+m_{2}$.

O que acabamos de ver é como a adição de dois momentos angulares definem um espaço composto que pode ser unicamente decomposto em autoespaços do momento angular total. A constribuição de Wigner a esse problema foi a determinação dos coeficientes explicitos para o conjunto de autovetores pertencentes a essa decomposição.

A derivação original de Wigner desses coeficientes usados em suas relações com as matrizes de rotação. Esse método é usualmente considerado mais como um grupo teorico do que alguns métodos usados depois que são baseados nas propriedades algébricas dos próprios operadores monentos angulares.

Existem vários métodos usados para calcular esses coeficientes, chamados de coeficientes de Wigner, ou também conhecidos como coeficientes de Clebsh-Gordan (ou ainda "vector-coupling"coeficientes), [5], essas várias abordagens de cálculo dos coeficientes 
de Wigner são indicativos da ampla extensão de interpretações e pontos de vistas que podem ser atribuidas ao aparatus matemático da teoria de momento angular.

Vamos dar continuidade a construção dada no inicio dessa seção. A expressão detalhada dos coeficientes de Wigner dependem do metodo de derivação. Todas tais fórmulas são equivalentes e podem ser transformadas umas nas outra por transformações simetricas e/ou pelo método de tranformação introduzido por Racah.

O método dos maiores pesos para determinação dos coeficientes de Wigner: o vetor ket em um multiplet $\mid(\alpha) j m>, m=j, j-1, \cdots,-j$, para o qual o peso $m$ é maximal, ou maior, isto é, $J_{+} \mid(\alpha) j m>=0$ para $j=m$, é chamado de vetor de maior peso. Uma vez que o vetor de maior peso é determinado, todo o multiplet é gerado pela aplicação sequencial do menor operador $J_{-}$.

Vamos denotar os vetores ket como um dos $j$-multiplets por $\mid\left(j_{1} j_{2}\right) j m>$. O vetor de maior peso é então denotado por $\mid\left(j_{1} j_{2}\right) j j>$ e o $j$-multiplet é dado por

$$
\left|\left(j_{1} j_{2} j m\right)>=\left[\frac{(j+m) !}{(2 j) !(j-m) !}\right]^{1 / 2} J_{-}^{j-m}\right|\left(j_{1} j_{2}\right) j j>.
$$

O vetor de maior peso, $\mid\left(j_{1} j_{2}\right) j j>$, para cada $j=j_{1}+j_{2}, j_{1}+j_{2}-1, \cdots,\left|j_{1}-j_{2}\right|$, é unicamente determinado por pertencer ao autovalor,$j$, de $J_{3}$ e sendo aniquilados pelo operador $J_{+}$:

$$
\begin{gathered}
\left|\left(j_{1} j_{2}\right) j j>=\sum_{m_{1} m_{2}, m_{1}+m_{2}=j} A_{m_{1} m_{2}}\right| j_{1} m_{1}>\mid j_{2} m_{2}>, \\
J_{+} \mid\left(j_{1} j_{2}\right) j j>=0 .
\end{gathered}
$$

Usando que $J_{+}=J_{+}(1)+J_{+}(2)$ e a ação do operador $J_{+}(i)$ sobre o vetor ket $\left|j_{i} m_{i}\right\rangle$, descrevemos agora dois termos de relação de recursão para os coeficietes $\left\{A_{m_{1} m_{2}}\right\}$, a solução que se encontra por iteração é

$$
A_{m_{1} m_{2}}=\delta\left(m_{1}+m_{2}, j\right)(-1)^{j_{1}-m_{1}}\left[\frac{\left(j_{1}+m_{1}\right) !\left(j_{2}+m_{2}\right) !}{\left(j_{1}-m_{1}\right) !\left(j_{2}-m_{2}\right) !}\right]^{1 / 2} A,
$$

onde $A$ é uma constante que é independente de $m_{1}$ e $m_{2}$.

A condição de normalização

$$
\sum_{m_{1} m_{2}, m_{1}+m_{2}=j}\left|A_{m_{1} m_{2}}\right|^{2}=1
$$


e a fórmula de soma

$$
\sum_{m_{1} m_{2}, m_{1}+m_{2}=j} \frac{\left(j_{1}+m_{1}\right) !\left(j_{2}+m_{2}\right) !}{\left(j_{1}-m_{1}\right) !\left(j_{2}-m_{2}\right) !}=\frac{\left(j+j_{1}+j_{2}+1\right) !\left(j+j_{1}-j_{2}\right) !\left(j-j_{1}+j_{2}\right) !}{(2 j+1) !\left(j-j_{1}+j_{2}\right)}
$$

nos fornecem o resultado desejado para o maior peso $j$ :

$$
\left|\left(j_{1} j_{2}\right) j j>=\sum_{m_{1} m_{2}} C_{m_{1} m_{2} j}^{j_{1} j_{2} j}\right| j_{1} m_{1}>\mid j_{2} m_{2}>,
$$

onde os coeficientes desse resultado é definido por:

$$
\begin{aligned}
C_{m_{1} m_{2} j}^{j_{1} j_{2} j}=\delta\left(m_{1}+\right. & \left.m_{2}, m\right)\left[\frac{(2 j+1) !\left(j-j_{1}+j_{2}\right)}{\left(j+j_{1}+j_{2}+1\right) !\left(j+j_{1}-j_{2}\right) !\left(j-j_{1}+j_{2}\right) !}\right]^{1 / 2} \\
& \times(-1)^{j_{1}-m_{1}}\left[\frac{\left(j_{1}+m_{1}\right) !\left(j_{2}+m_{2}\right) !}{\left(j_{1}-m_{1}\right) !\left(j_{2}-m_{2}\right) !}\right]^{1 / 2} .
\end{aligned}
$$

O $j$-multiplet pode agora ser escrito da seguinte forma:

$$
\left|\left(j_{1} j_{2}\right) j m>=\sum_{m_{1} m_{2}} C_{m_{1} m_{2} j}^{j_{1} j_{2} j}\right| j_{1} m_{1}>\mid j_{2} m_{2}>
$$

onde

$$
\begin{gathered}
C_{m_{1} m_{2} j}^{j_{1} j_{2} j} \equiv\left[\frac{(j+m) !}{(2 j) !(j-m) !}\right]^{1 / 2}<j_{1} m_{1} ; j_{2} m_{2}\left|J_{-}^{j-m}\right|\left(j_{1} j_{2}\right) j j> \\
\equiv\left[\frac{(j+m) !}{(2 j) !(j-m) !}\right]^{1 / 2}<j_{1} j 2 j j\left|J_{+}^{j-m}\right| j_{1} m_{1} ; j_{2} m_{2}>
\end{gathered}
$$

no qual $\left|j_{1} m_{1} ; j_{2} m_{2}>\equiv\right| j_{1} m_{1}>\mid j_{2} m_{2}>$.

O metodo de cálculo de Wigner fornece os seguintes coeficientes:

$$
\begin{aligned}
C_{m_{1} m_{2} j}^{j_{1} j_{2} j}=\delta\left(m_{1}\right. & \left.+m_{2}, m\right)\left[\frac{(2 j+1)\left(j+j_{1}-j_{2}\right) !\left(j-j_{1}+j_{2}\right) !\left(-j+j_{1}+j_{2}\right)}{\left(j+j_{1}+j_{2}+1\right) !}\right]^{1 / 2} \\
& \times\left[\frac{(j+m) !(j-m) !}{\left(j_{1}+m_{1}\right) !\left(j_{1}-m_{1}\right) !\left(j_{2}+m_{2}\right) !\left(j_{2}-m_{2}\right) !}\right]^{1 / 2} \\
& \times \sum_{s} \frac{(-1)^{j_{2}+m_{2}+s}\left(j+j_{2}+m_{1}-s\right) !\left(j_{1}-m_{1}+s\right)}{s !\left(j-j_{1}+j_{2}-s\right) !(j+m-s) !\left(j_{1}-j_{2}-m+s\right) !} .
\end{aligned}
$$

Ortogonalidade dos coeficientes de Wigner: como os vetores kets no conjunto: 


$$
\left\{\left|\left(j_{1} j_{2}\right) j m>: j=j_{1}+j_{2}, j_{1}+j_{2}-1, \cdots,\right| j_{1}-j_{2} \mid ; m=j, j-1, \cdots,-j\right\}
$$

são ortonormais os coeficientes de Wigner são elementos de uma matriz real ortogonal $C$ de dimensão $\left(2 j_{1}+1\right)\left(2 j_{2}+1\right)$, expressando uma mudança de base no espaço tensorial $H_{j_{1}} \otimes H_{j_{2}}$. Essa mudança de base divide as matrizes momento angular $M_{i}$, dada por $J_{i} \rightarrow M_{i}=J^{\left(j_{1}\right)} \otimes 1^{\left(j_{2}\right)}+1^{\left(j_{1}\right)} \otimes J^{\left(j_{2}\right)}$ nas matrizes momento angular $J^{(j)}$ :

$$
\begin{aligned}
& C J^{\left(j_{1}\right)} \otimes 1^{\left(j_{2}\right)}+1^{\left(j_{1}\right)} \otimes J^{\left(j_{2}\right)} \widetilde{C} \sum_{j} \oplus J^{(j)} \\
& =\left(\begin{array}{cccc}
J_{i}^{\left(j_{1}+j_{2}\right)} & 0 & \cdots & 0 \\
0 & J_{i}^{\left(j_{1}+j_{2}-1\right)} & & 0 \\
\vdots & & \ddots & \vdots \\
0 & 0 & \cdots & J_{i}^{\left(\left|j_{1}-j_{2}\right|\right)}
\end{array}\right) .
\end{aligned}
$$

Os elementos de $C$ são identificados explicitamente para serem

$$
(C)_{j m ; m_{1} m_{2}}=C_{m_{1} m_{2} m}^{j_{1} j_{2} j}
$$

onde os índices pares $(\mathrm{jm})$ e $\left(m_{1} m_{2}\right)$ enumeram linhas e colunas respectivamente. Portanto, as $\left(2 j_{1}+1\right)\left(2 j_{2}+1\right)$ linhas e colunas são enumeradas, respectivamente, por

$$
\begin{gathered}
(j m): j=j_{1}+j_{2}, \cdots,\left|j_{1}-j_{2}\right| ; m=j, \cdots,-j \\
\left(m_{1} m_{2}\right): m_{1}=j_{1}, \cdots,-j_{1} ; m_{2}=j_{2}, \cdots,-j_{2} .
\end{gathered}
$$

A seguir as afirmações completas sobre a ortogonalidade de $C$ :

(a) Ortogonalidade das linhas:

$$
\sum_{m_{1} m_{2}} C_{m_{1} m_{2} m}^{j_{1} j_{2} j} C_{m_{1} m_{2} m^{\prime}}^{j_{1} j_{2} j^{\prime}}=\delta_{j j^{\prime}} \delta_{m m^{\prime}}
$$

(b) Ortogonalidade das colunas:

$$
\sum_{j m} C_{m_{1} m_{2} m}^{j_{1} j_{2} j} C_{m_{1}^{\prime} m_{2}^{\prime} m}^{j_{1} j_{2} j^{\prime}}=\delta_{m_{1} m_{1}^{\prime}} \delta_{m_{2} m_{2}^{\prime}}
$$


Devido ao fato que alguns dos elemetnos de $C$ serem nulos, a afirmação para a relação de ortogonalidade podem ser substancialmente simplificadas removendo as situações triviais $0=0$. Todas as relações são consequências de

$$
C_{m_{1} m_{2} m}^{j_{1} j_{2} j}=0 \quad m_{1}+m_{2} \neq m,
$$

a qual é ela mesma consequência do fato de $J_{3}=J_{3}(1)+J_{3}(2)$ possuir autovalor $m$ quando atuando em $\left|j_{1} m_{1}>\right| j_{2} m_{2}>$.

Contabilizando para esses zeros na matriz $C$, a ortogonalidade das linhas se reduz a

$$
\sum_{m_{1} m_{2}, m_{1}+m_{2}=m} C_{m_{1} m_{2} m}^{j_{1} j_{2} j} C_{m_{1} m_{2} m}^{j_{1} j_{2} j^{\prime}}=\delta_{j j^{\prime}}
$$

enquanto que a ortogonalidade das colunas fica melhor escrita em duas formas:

$$
\begin{aligned}
& \sum_{j} C_{m_{1}, m-m_{1}, m}^{j_{1} j_{2} j} C_{m_{1}^{\prime}, m-m_{1}^{\prime}, m}^{j_{1} j_{2} j^{\prime}}=\delta_{m_{1} m_{1}^{\prime}}, \\
& \sum_{j} C_{m_{1}, m-m_{1}, m}^{j_{1} j_{2} j} C_{m_{1}^{\prime}, m-m_{1}^{\prime}, m}^{j_{1} j_{2} j^{\prime}}=\delta_{m_{1} m_{1}^{\prime}},
\end{aligned}
$$

Para levar em conta as mudanças de fases e fatores de dimensionalidade, vamos definir um novo símbolo que corresponde o mais próximo possível das simetrias dos coeficientes de Wigner. Esse é o $3 j$ símbolo, introduzido por Wigner e definido como:

$$
\begin{gathered}
\left(\begin{array}{ccc}
j_{1} & j_{2} & j \\
m_{1} & m_{2} & -m
\end{array}\right) \equiv(-1)^{j_{1}-j_{2}+m}(2 j+1)^{-1 / 2} C_{m_{1} m_{2} m}^{j_{1} j_{2} j} \\
\quad \equiv R\left[\begin{array}{ccc}
j_{1}+m_{1} & j_{2}+m_{2} & j-m \\
j_{1}-m_{1} & j_{2}-m_{2} & j+m \\
j-j_{1}+j_{2} & j+j_{1}-j_{2} & -j+j_{1}+j_{2}
\end{array}\right]
\end{gathered}
$$

A segunda linha da equação acima define a notação de Regge para o $3 j$-simbolo em termos da matriz Regge.

As propriedades de simetrias agora possuem uma elegante expressão em termos da simetria do determinante da matriz Regge, portanto, o simbolo de Regge é invariante sobre todas as permutações pares de suas linhas e colunas, e sobre transposições da matriz e é multiplicada pelo fator $(-1)^{j_{1}+j_{2}+j}$ sobre as permutações ímpares de suas linhas e colunas.

Algumas situações: 


$$
\begin{gathered}
\left(\begin{array}{ccc}
j_{1} & j_{2} & j \\
m_{1} & m_{2} & m
\end{array}\right)=\left(\begin{array}{ccc}
j_{2} & j & j_{1} \\
m_{2} & m & m_{1}
\end{array}\right)=\left(\begin{array}{ccc}
j & j_{1} & j_{2} \\
m & m_{1} & m_{2}
\end{array}\right) \\
(-1)^{j_{1}+j_{2}+j}\left(\begin{array}{ccc}
j_{2} & j_{1} & j \\
m_{2} & m_{1} & m
\end{array}\right)=(-1)^{j_{1}+j_{2}+j}\left(\begin{array}{ccc}
j_{1} & j & j_{2} \\
m_{1} & m & m_{2}
\end{array}\right) \\
=(-1)^{j_{1}+j_{2}+j}\left(\begin{array}{ccc}
j & j_{2} & j_{1} \\
m & m_{2} & m_{1}
\end{array}\right)=(-1)^{j_{1}+j_{2}+j}\left(\begin{array}{ccc}
j_{1} & j_{2} & j \\
-m_{1} & -m_{2} & -m
\end{array}\right) .
\end{gathered}
$$

\subsection{Coeficientes de Racah e 6j-simbolo.}

Em torno de 60 anos atrás os W-coeficientes apareciam pela primeira vez no artigo de Racah [18] como uma ferramenta auxiliar para o cálculo dos elementos matriciais na teoria espectral complexa. Hoje não há qualquer ramo da física, que envolva momento angular, onde o uso dos W-coeficientes não seja necessário afim de realizar o mais simples cálculo. Vemos que os W-coeficientes são algo a mais do que simplesmente uma ferramente útil para os fisicos teóricos usá-la. De fato, um completo entendimento das propriedades dessa função pode muito bem ceder a uma nova visão na teoria de momento angular.

Segundo [17], uma completa investigação ao limite semiclassico dos W-coeficientes e funções relacionadas são pré requisitos para o entendimento profundo de suas propriedades

Os W-coeficientes, ou a versão simétrica, $6 j$-símbolo definida por Wigner [23], tem sido por anos uma pratica normal associar a este um diagrama, ou gráfico, que exibe propriedades de simetria de maneira clara. Uma vantagem para esse diagrama é que pode ser generalizado ordem maior, $3 j n$-simbolo definido também por Wigner e outros. Existem pelo menos, três versões diferentes desses algoritmos gráficos, todos tendo aproximadamente o mesmo conteúdo.

Os coeficientes de Racah, $W\left(j_{1} m_{2} j_{2} m_{2} ; j_{3} m_{3}\right)$, são "recoupling"de três momentos angulares e mais importante, eles constituem uma base para o "recouling"coeficientes de adição de um número arbitrário de momentos angulares, [6].

Do ponto de vista matemático, o estudo dos coeficientes de Racah é interessante por várias razões:

(a) são objetos básicos independentes, ou seja, são independentes do números quânticos projeção $(m)$; 
(b) os próprios coeficientes de Wigner podem ser obtidos como o limite dos coeficientes de Racah;

(c) são generalizações de séries hipergeométricas;

(d) estão relaciondas com a geometria projetiva;

(e) eles definem uma algebra. (Todos os itens estão bem desenvolvidos em [6], o que não faremos aqui, pois foge do contexto da tese.)

O $6 j$-simbolo de Wigner é denotado por

$$
\left\{\begin{array}{ccc}
j_{1} & j_{2} & j_{3} \\
m_{1} & m_{2} & m_{3}
\end{array}\right\}
$$

onde $j_{1}, j_{2}, j_{3}, m_{1}, m_{2}, m_{3}$ são inteiros ou meio-inteiros e cujas triplas $\left(j_{1}, j_{2}, j_{3}\right),\left(m_{1}, j_{2}, j_{3}\right)$, $\left(j_{1}, m_{2}, m_{3}\right),\left(m_{1}, m_{2}, j_{3}\right)$ devem satisfazer:

1) Cada tripla satisfaz a desigualdade triangular: $\left|j_{i}-j_{k}\right| \leq j_{l} \leq j_{i}+j_{k}$, para as triplas $(i, k, l)$;

2) a soma dos elementos de cada tripla é um inteiro. Portanto, os elementos de cada tripla são ou todos inteiros ou contem pelo menos dois meio inteiros e um inteiro.

Se essas condições não forem satisfeitas, então:

$$
\left\{\begin{array}{ccc}
j_{1} & j_{2} & j_{3} \\
m_{1} & m_{2} & m_{3}
\end{array}\right\}=0
$$

A relação entre os coeficientes de Racah e $6 j$-símbolo é dada por:

$$
\left\{\begin{array}{ccc}
j_{1} & j_{2} & j_{3} \\
m_{1} & m_{2} & m_{3}
\end{array}\right\}=(-1)^{j_{1}+m_{1}+j_{2}+m_{3}} W\left(j_{1} m_{2} j_{2} m_{2} ; j_{3} m_{3}\right) .
$$

Algumas propriedades do $6 j$-simbolo:

(a) invariante por permutação de duas colunas:

$$
\left\{\begin{array}{ccc}
j_{1} & j_{2} & j_{3} \\
m_{1} & m_{2} & m_{3}
\end{array}\right\}=\left\{\begin{array}{ccc}
j_{2} & j_{1} & j_{3} \\
m_{2} & m_{1} & m_{3}
\end{array}\right\}
$$

(b) invariante sobre a troca de dois elementos correspondentes em linhas:

$$
\left\{\begin{array}{ccc}
j_{1} & j_{2} & j_{3} \\
m_{1} & m_{2} & m_{3}
\end{array}\right\}=\left\{\begin{array}{ccc}
m_{1} & m_{2} & j_{3} \\
j_{1} & j_{2} & m_{3}
\end{array}\right\}
$$

O $6 j$-simbolo pode ser calculado a partir da fórmula que usa o coeficietne triangular: 


$$
\begin{gathered}
\left\{\begin{array}{ccc}
j_{1} & j_{2} & j_{3} \\
m_{1} & m_{2} & m_{3}
\end{array}\right\}=\sqrt{\Delta\left(j_{1}, j_{2}, j_{3}\right) \Delta\left(j_{1}, m_{2}, m_{3}\right) \Delta\left(m_{1}, j_{2}, m_{3}\right) \Delta\left(m_{1}, m_{2}, j_{3}\right)} \\
\times \sum_{t} \frac{(-1)^{t}(t+1) !}{f(t)}
\end{gathered}
$$

onde $\Delta(a, b, c)$, dado por,

$$
\Delta(a, b, c)=\frac{(-a+b+c) !(a-b+c) !(a+b-c) !}{(a+b+c+1) !}
$$

é o coeficiente triangular e

$$
\begin{gathered}
f(t)=\left(t-j_{1}-j_{2}-j_{3}\right) !\left(t-j_{1}-m_{2}-m_{3}\right) !\left(t-m_{1}-m_{2}-j_{3}\right) ! \\
\left(j_{1}+j_{2}+m_{1}+m_{2}-t\right) !\left(j_{2}+j_{3}+m_{2}+m_{2}-t\right) !\left(j_{3}+j_{1}+m_{3}+m_{1}-t\right) !
\end{gathered}
$$

e a soma será sobre todos os inteiros $t$ para os quais os fatoriais em $f(t)$ não possuam argumentos negativos.

Para mais propriedades e outras fórmulas para os $3 j$ e $6 j$ símbolos de Wigner consultar $([21])$.

Como iremos usar a idéia de [17] no capítulo 3, adotaremos igualmente o tetraedro para a representação espacial do $6 j$-símbolo. Diagrama descrito com mais detalhes no Apendice B. 


\begin{tabular}{l|c|}
\hline & Capítulo \\
\cline { 2 - 2 } & 2 \\
\hline
\end{tabular}

\section{Os trikernels de Stratonovich e de Berezin}

O objetivo desse capítulo é exibir uma fórmula fechada, que dependa de expressões invariantes por rotações, ou seja, que dependa de produtos internos e determinantes, para o trikernel de Stratonovich e de Berezin, no caso geral de $n=2 j$. Como veremos adiante, produtos de funções na esfera, induzidos pelo produto de matrizes (operadores), podem ser escritos na forma integral,

$$
f \star^{n} g=\iint_{S^{2} \times S^{2}} f g L^{j}
$$

onde $L^{j}: S^{2} \times S^{2} \times S^{2} \rightarrow \mathbb{C}$ é o trikernel integral que define o produto $\star$. Conforme exposto em [12] e resumido adiante, há várias escolhas possíveis para $L^{j}$, mas duas se destacam: o trikernel padrão de Stratonovich-Weyl e o trikernel padrão de Berezin.

No artigo de Varilly e Gracia-Bondía [20] é apresentado como um exemplo a fórmula recursiva para o trikernel de Stratonovich-Weyl:

$$
L^{1 / 2}\left(n_{1}, n_{2}, n_{3}\right)=\frac{1}{4}\left(1+3\left(<n_{1}, n_{2}>+<n_{2}, n_{3}>+<n_{1}, n_{3}>\right)+3 \sqrt{3} i\left[n_{1}, n_{2}, n_{3}\right]\right)
$$


onde $<$, > é o produto interno em $\mathbb{R}^{3}$ e $[., .,$.$] é o determinante da matriz 3 \times 3$, consituida dos vetores $n, m, k$. Mas não há na literatura outros exemplos calculados.

Além de calcular os exemplos $j=1, \frac{3}{2}$ e 2 para o trikernel de Stratonovich e de Berezin, cogitou-se a hipótese de haver uma fórmula fechada válida para todo $j$. Indo em busca dessa fórmula concluímos, como veremos nesse capítulo, que não foi possível obter tal qualidade de fórmula. Porém, essa nova expressão, para valores mais baixos de $j$, ficou em função de produtos internos e determinantes.

A seguir apresentaremos, baseando-se no trabalho de Pedro M. Rios e Eldar Straume, [12], toda a nomenclaruta, definições e operadores citados acima com suas fórmulas trabalhadas nesse preprint. E depois dessa apresentação, partimos ao objetivo principal.

\subsection{Correspondência de símbolos para sistemas de spin.}

As definições e o contexto apresentados a seguir são baseados fortemente em [12] e [20], pois são trabalhos que apresentam claramente o que o artigo de Stratonovich [19] não o faz, em vista de que com o passar dos anos o assunto introduzido por Stratonovich veio sendo estudado mais a fundo.

Na formulação comum da mecânica quantica, o $j$-spin é representado por operadores atuando num espaço vetorial $\mathbb{C}^{2 j+1}$, onde $j \in\{1 / 2,1,3 / 2, \ldots\}$. Uma base ortonormal é dada por autovetores $\mid j m>$, onde

$$
\begin{gathered}
J^{2}|j m>=j(j+1)| j m>, \\
J_{3}|j m>=m| j m>,
\end{gathered}
$$

colocando a constante de Planck $\hbar=1$.

$\mathbb{C}^{2 j+1}$ carrega uma representação irredutivel $\pi_{j}$ de $S U(2)$ cujos elementos matriciais são convencionalmente dados por

$$
D_{m n}^{j}(g):=<j m\left|\pi_{j}(g)\right| j m>.
$$

O espaço de fase é a esfera $S^{2}$ equipada com a forma simplética $S U(2)$-invariante $\sin \varphi d \varphi \wedge d \vartheta$; esse fato familiar pode ser visto como aplicação do teorema KostantKirilov-Souriau para o grupo $S U(2)$, pois suas órbitas coadjuntas são esferas e $S U(2)$ atua em cada órbita por rotações da esfera.

Denotamos pontos da esfera por $n=(\varphi, \vartheta)$ em cordenadas esfericas; $d n:=\sin \varphi d \varphi \wedge$ $d \vartheta$ é a medida de area da superficie. Elementos de $S U(2)$ serão genericamente deno- 
tados por $g$ e a ação natural de $S U(2)$ em $S^{2}$ será denotada por $g \cdot n$, efetivamente uma ação de $S O(3)$. Se $f$ é uma função na esfera, $f^{g}$ é dada por $f^{g}(n):=f\left(g^{-1} \cdot n\right)$. A medida de Haar $d g$ sobre $S U(2)$ é normalizada de maneira que $\int_{S U(2)} d g=1$. Para mais fatos e detalhes sobre momento angular spin em mecânica quantica, indicamos [5].

Definição 2.1.1. Um sistema quantico $j$-spin é um espaço de Hilbert $H_{j} \simeq \mathbb{C}^{n+1}$ munido de uma representação unitária irredutivel

$$
\pi_{j}: S U(2) \rightarrow G \subset U(2 j+1)
$$

onde $n=2 j, G$ denota a imagem de $S U(2)$ que por usa vez é isomorfa a $S U(2)$ ou $S O(3)$ dependendo se $j$ for semi-inteiro ou inteiro.

Ainda segundo [12]:

Definição 2.1.2. Uma correspondência de símbolos para um sistema quantico j-spin $\mathcal{H}_{j} \simeq \mathbb{C}^{n+1}$, onde $n=2 j$, é uma regra a qual associa a cada operador $P \in \mathcal{B}\left(\mathcal{H}_{j}\right)$ uma função suave $W_{P}$ sobre o espaço fase $\mathcal{S}^{2}$ com as seguintes propriedades:

(i) Linearidade: $A$ aplicação $P \rightarrow W_{P}$ é linear e injetiva.

(ii) Equivariancia: $W_{P^{g}}=\left(W_{P}\right)^{g}$, para $g \in S U(2)$.

(iii) Realidade: $\overline{W_{P^{*}}^{j}(n)=W_{P}^{j}(n)}$.

(iv) Normalização: $\frac{1}{4 \pi} \int_{S^{2}} W_{P}^{j} d S=\frac{1}{n+1} \operatorname{Tr}(P)$.

Desta maneira caracterizamos uma família de aplicações de correspondência de símbolos, que será sobrejetora ao restringirmos o contra-domínio desta:

$$
\begin{gathered}
W^{j}: B\left(H_{j}\right) \simeq M_{\mathbb{C}}(n+1) \rightarrow \operatorname{Poly}_{\mathbb{C}}^{m}\left(S^{2}\right) \subset \mathcal{C}_{\mathbb{C}}^{\infty}\left(S^{2}\right), \\
P \mapsto W_{P}^{j},
\end{gathered}
$$

onde Poly $y_{\mathbb{C}}^{m}$ é o conjunto dos polinômios de grau menor ou igual a $m$ e $W^{j}$ associa cada operador $P$ a seu símbolo $W_{P}^{j}$. Assumimos que os símbolos $W_{P}^{j}$ são funções polinomiais.

Notamos que por $(i)$ e (ii) resulta que $W^{j}$ é um $G$-isomorfismo e (iii) assegura que $W^{j}$ respeita a estrutura real. Portanto, pelo seguinte teorema ${ }^{1}$,

\footnotetext{
${ }^{1}$ para demonstração vide [12].
} 
Teorema 2.1.3. Cada $G$-aplicação $\mathbb{R}$ - linear que leva matrizes herminitanas em polinômios reais pode ser identificada como uma única $(n+1)$-upla

$$
\left(c_{0}, c_{1}, \cdots, c_{n}\right) \in \mathbb{R}^{n+1} .
$$

Em particular, essa upla corresponde a um G-isomorfismo

$$
\begin{aligned}
H(n+1) & \simeq \operatorname{Poly}_{\mathbb{R}}\left(S^{2}\right)_{\leq n} \\
\cap & \cap \\
M_{C}(n+1) & \simeq \operatorname{Poly}_{\mathbb{C}}\left(S^{2}\right)_{\leq n}
\end{aligned}
$$

se e somente se cada $c_{i} \neq 0$.

$W^{j}$ é representado pela $(n+1)$-upla real, $\left(c_{0}, c_{1}, \cdots, c_{n}\right)$, com cada $c_{l} \neq 0$.

Observamos que o símbolo $W_{I}^{j}$ do operador identidade $I$, é uma função constante, digamos, $c_{0}$. Junto com a condição $(i v)$, implica que $c_{0}=1$. Nesse sentido, podemos identificar cada símbolo $W^{j}$ com sua $n$-upla:

$$
W^{j} \leftrightarrow\left(c_{1}, \cdots, c_{n}\right) \in\left(R^{*}\right)^{n}
$$

onde $R^{*}=R-\{0\}$.

Junto com os axiomas acima podemos impor mais um:

$$
\text { (v) Isometria: } \quad<W_{P}^{j}, W_{Q}^{j}>=<P, Q>_{j}
$$

como uma condição de "normalização métrica"onde o lado direito da equação acima é o produto interno normalizado de Hilbert-Schmidt de dois operadores, dado por

$$
<P, Q>_{j}=\frac{1}{n+1}<P, Q>=\frac{1}{n+1} \operatorname{Tr}\left(P^{*} Q\right)
$$

$n=2 j$ e o lado esquerdo é o produto interno em $L^{2}$ de duas funções, dada por

$$
<W_{P}^{j}, W_{Q}^{j}>=\frac{1}{4 \pi} \int_{S^{2}} \overline{W_{P}^{j}(n)} W_{Q}^{j}(n) d S .
$$

Portanto a condição (iv) é apenas um caso especial de $(v)$, ou seja, (iv) pode ser apresentada como,

$$
<1, W_{P}^{j}>=<I, P>_{j} .
$$

O primeiro a investigar a correspondência de símbolos para sistemas de spin foi Berezin em [3], [4] e [2]. Sua correspondência satisfaz os axiomas $(i)-(i v)$, mas não 
o axioma $(v)$. Varilly e Gracia-Bondia [20], foram os primeiros a estudar sistematicamente o tipo de correspondência $P \leftrightarrow W_{P}$ que satisfaz todos os cinco axiomas, como introduzido anteriormente por Stratonovich em [19], generalizando para sistemas $j$-spin a correspondência de Weyl.

Definição 2.1.4. Uma correspondência de Stratonovich-Weyl é uma correspondência de símbolos que satisfaz também o axioma $(v)$.

\subsubsection{Correspondência de símbolos via operador kernel, o operador kernel de Stratonovich-Weyl e de Berezin.}

Seguindo a idéia de Várilly e Garcia-Bondía [20], temos que por linearidade, escrevemos:

$$
W_{P}(n)=\operatorname{tr}\left(P \Delta^{j}(n)\right)
$$

onde $\Delta^{j}$ é um operador sobre $S^{2}$, o qual chamamos de operador Kernel de Stratonovich Weyl. Agora a propriedade de tracialidade nos fornece que

$$
P=\frac{2 j+1}{4 \pi} \int_{S^{2}} W_{P}(n) \Delta^{j}(n) d n .
$$

Em outras palavras, a correspondencia direta e inversa, $P \rightleftarrows W_{P}$, devem ser implementadas pelo mesmo operador kernel. E de fato, tal operador existe.

A construção de uma aplicação de símbolo $W^{j}$ em termos de um operador kernel exigirá algumas particularidades. De forma geral, vamos chamar esse operador de $K$, onde $K \in M_{\mathbb{C}}(n+1)$, que fornecerá uma função em $S^{2}$. Sendo $n_{0}=(0,0,1), n=2 j$, $K\left(n_{0}\right)=K$ e $K(n)=K\left(g n_{0}\right)=K^{g}$ para $n=g n_{0}$, temos a seguinte relação [12]:

Proposição 2.1.5. Para cada correspondência de símbolo $W$ existe um único operador $K \in M_{\mathbb{C}}(n+1)$ tal que

$$
W_{P}^{j}\left(g n_{0}\right)=\operatorname{Tr}\left(P K^{g}\right)
$$

ou, equivalentemente,

$$
W_{P}^{j}(n)=\operatorname{Tr}(P K(n))
$$

Teremos que, além disso, $K$ será uma matriz diagonal de traço 1. 
Demonstração: De fato, o funcional linear

$$
\begin{aligned}
& \widehat{W}: M_{\mathbb{C}}(n+1) \rightarrow \mathbb{C}, \\
& P \mapsto \widehat{W}(P)=W_{P}\left(n_{0}\right)
\end{aligned}
$$

é representado por algum $K$ de maneira que $\widehat{W}(P)=\operatorname{Tr}(P K)$. Portanto, tomando $g=1$, temos $W_{P}\left(n_{0}\right)=\operatorname{Tr}(P K)$. E generalizando,

$$
W_{P}\left(g^{-1} n_{0}\right)=\left(W_{P}\right)^{g}\left(n_{0}\right)=W_{P^{g}}\left(n_{0}\right)=\operatorname{Tr}\left(P^{g} K\right)=\operatorname{Tr}\left(P K^{g^{-1}}\right) .
$$

Por outro lado, para $g \in U(1)$, temos $\operatorname{Tr}(P K g)=\operatorname{Tr}(P K)$, para todo $P$. consequentemente, $K^{g}=K$, isto é, $K$ é fixado por $U(1)$ e por isso, $K=\operatorname{diag}\left(\lambda_{1}, \lambda_{2}, \cdots, \lambda_{n+1}\right)$. Escolhendo $P$ os elementos da base de $M_{\mathbb{C}}(n+1), E_{k k}$, obtemos $\lambda_{k}=W_{P}\left(n_{0}\right)$ é um número real, devido a condição de realidade, $(i i)$. Finalmente pela condição de normallização, $($ iii $), W_{I}=1$ e portanto, $\operatorname{Tr}(K)=1$.

Agora estamos aptos a apresentar a seguinte:

Definição 2.1.6. Um operador kernel $K \in M_{\mathbb{C}}(n+1)$ é uma matriz diagonal com a propriedade de que uma aplicação de símbolo dada por

$$
W_{P}^{j}\left(g n_{0}\right)=\operatorname{Tr}\left(P K^{g}\right)
$$

é uma correspondência de símbolo.

Em vista do que apresentamos acima, $K$ possui uma decomposição ortogonal

$$
K=\frac{1}{n+1} I+K_{1}+K_{2}+\cdots+K_{n}
$$

onde para $l \geq 1, K_{l}$ é uma matriz diagonal, real e de traço nulo, ou seja, $K_{l}=k_{l} e(l, 0)$, onde $k_{l} \neq 0$ e

$$
e(l, 0)=\frac{(-1)^{l}}{l !} \sqrt{2 l+1} \sqrt{\frac{(n-l) !}{(n+l+1) !}} \sum_{k=0}^{l}(-1)^{k}\left(\begin{array}{c}
l \\
k
\end{array}\right) J_{-}^{l-k} J_{+}^{l} J_{-}^{k},
$$


onde

$$
J_{-}=J_{+}^{t}=\left[\begin{array}{cccccc}
0 & 0 & 0 & \cdots & 0 & 0 \\
\sqrt{n .1} & 0 & 0 & \cdots & 0 & 0 \\
0 & \sqrt{(n-1) .2} & 0 & \cdots & 0 & 0 \\
\vdots & \vdots & \vdots & \vdots & \vdots & \vdots \\
0 & \cdots & 0 & \sqrt{2 .(n-1)} & 0 & 0 \\
0 & 0 & \cdots & 0 & \sqrt{1 . n} & 0
\end{array}\right]
$$

que coincidem com $J_{-}$e $J_{+}$apresentadas no capitulo 1 , secção 1.3.

Proposição 2.1.7. Existe uma bijeção entre correspondência de símbolos e matrizes reais diagonais em $M_{C}(n+1)$ do tipo

$$
K=\frac{1}{n+1} I+\sum_{l=1}^{n} c_{l} \sqrt{\frac{2 l+1}{n+1}} e(l, 0), \quad c_{l} \neq 0, \quad \text { real },
$$

e ainda, para toda correspondência de símbolos de Stratonovich-Weyl, $c_{l}= \pm 1$ para todo $l$.

Demonstração: vide [12].

Definição 2.1.8. Os números reais não nulos $c_{1}, c_{2}, \ldots, c_{n}$ são denominados números característicos do operador kernel $K$.

Definição 2.1.9. A única correspondência positiva de símbolos de Stratonovich-Weyl (para a qual todos os números característicos são iguais a 1 , isto é, $c_{l}=1$ ) é chamada de correspondência de símbolos de Stratonovich-Weyl padrão e denotada por:

$$
W_{1}^{j}: B\left(H_{j}\right) \simeq M_{\mathbb{C}}(n+1) \rightarrow \operatorname{Poly}_{\mathbb{C}}\left(S^{2}\right)_{n \leq} \subset C_{\mathbb{C}}^{\infty}\left(S^{2}\right)
$$

O operador kernel $K$, neste caso, é denotado por $K_{1}$ ou por $\Delta$ (como em [20]).

Vamos agora destacar a correspondencia de símbolos que originalmente foi definida por Berezin.

Definição 2.1.10. A correspondência de símbolo de Berezin é uma correspondencia de símbolos cujo operador kernel $K$ é um operador projeção $\Pi$.

Como o traço de um operador projeção é seu rank, segue que П deve ser uma matriz $\Pi_{k}=E_{k k}$ para $1 \leq k \leq n+1=2 j+1$, ou seja,

$$
\Pi_{k}=(-1)^{k+1} \sum_{l=0}^{n} C_{m,-m, 0}^{j, j, l} e(l, 0),
$$


onde $m=j-k+1$ e cada coeficiente de Clebsch-Gordan deve ser não nulo.

Definição 2.1.11. A correspondência de símbolo obtida via operador projeção $\Pi_{1}$ é chamado de símbolo de Berezin padrão.

Assim como definimos uma correspondência de símbolos via um kernel para o caso Stratonovich, podemos fazer o mesmo para Berezin da seguinte maneira: definimos uma aplicação $B$ que associa a cada operador $Q \in M_{\mathbb{C}}(n+1)$ a função $B_{Q}$ sobre a esfera $S^{2}$,

$$
B_{Q}\left(g n_{0}\right)=\operatorname{Tr}\left(Q \Pi_{1}^{g}\right), \quad g \in S U(2),
$$

onde $n_{0}=(0,0,1) \in S^{2}$ e $\Pi_{1}$ é o operador projeção

$$
\Pi_{1}=\operatorname{diag}(1,0,0, \cdots, 0) .
$$

Definição 2.1.12. A aplicação B é a correspondência de símbolo de Berezin padrão.

Os números característicos para a correspondência de símbolo de Berezin padrão são os números característicos da aplicação de símbolo cujo operador kernel é o operador projeção $\Pi_{1}$. Portanto para cada $n=2 j$ existe uma $n$-upla $\vec{b}$ de $n$ números característicos possivelmente dependendo de $n$. Denotaremos esses números por $b_{l}^{n}$,

$$
\vec{b}=\left(b_{1}^{n}, b_{2}^{n}, \cdots, b_{l}^{n}, \cdots, b_{n}^{n}\right)
$$

De acordo com 2.1.1, estes números são expressados da seguinte maneira, pelo seguinte produto interno:

$$
b_{l}^{n}=\sqrt{\frac{n+1}{2 l+1}}<\Pi_{1}, e^{j}(l, 0)>=\sqrt{\frac{n+1}{2 l+1}} e^{j}(l, 0)_{1,1},
$$

onde $e^{j}(l, 0)_{1,1}$ denota a primeira entrada da matriz diagonal $e^{j}(l, 0)=e(l, 0)$.

A forma explícita dos números característicos $b_{l}^{n}$ da correspondência de símbolos padrão de Berezin é dada por: 


$$
\begin{array}{r}
b_{l}^{n}=\sqrt{\frac{n(n-1) \ldots(n-l+1)}{(n+2)(n+3) \ldots(n+l+1)}} \\
=\sqrt{\frac{\left(\begin{array}{c}
n \\
l
\end{array}\right)}{\left(\begin{array}{c}
n+l+1 \\
l
\end{array}\right)}=\frac{n ! \sqrt{n+1}}{\sqrt{(n+l+1) !(n-l) !}}} .
\end{array}
$$

Observamos que essa fórmula é obtida a partir de

$$
b_{l}^{n}=\sqrt{\frac{n+1}{2 l+1}} C_{j,-j, 0}^{j, j, l} .
$$

Mas há uma outra demonstração de como encontrar esses $b_{l}^{n}$ bastante interessante que pode ser vista no Apendice 7.4 de [12].

\subsection{Produto Twisted na Esfera}

Dada uma correspondência de símbolos, definimos:

$$
W_{A} \star W_{B}=W_{A B},
$$

para quaisquer operadores $A$ e $B$. Dada a correspondencia de Stratonovich Weyl, para um particular $j$, notamos que:

$$
\begin{gathered}
W_{A B}=\operatorname{Tr}\left(\Delta^{j}(n) A B\right) \\
=\left(\frac{2 j+1}{4 \pi}\right)^{2} \operatorname{Tr}\left(\Delta^{j}(n) \int_{S^{2}} \Delta^{j}(m) W_{A}(m) d m \int_{S^{2}} \Delta^{j}(k) W_{B}(k) d k\right) .
\end{gathered}
$$

Portanto a definição apropriada para o produto twisted de duas funções $g$ e $f$ em $\mathfrak{H}_{2 j}{ }^{2}$, é dado por

$$
\left(f \star_{1} g\right)(n):=\int_{S^{2}} \int_{S^{2}} L_{1}^{j}(n, m, k) f(m) g(k) d m d k,
$$

onde o trikernel $L_{1}^{j}$ é apenas

\footnotetext{
${ }^{2}$ Espaço de Hilbert dos harmônicos esféricos cuja base ortonormal é $\left\{Y_{l}^{m}: 0 \leq l \leq 2 j,-l \leq m \leq l\right\}$.
} 


$$
L_{1}^{j}(n, m, k)=\left(\frac{2 j+1}{4 \pi}\right)^{2} \operatorname{tr}\left(\Delta^{j}(n) \Delta^{j}(m) \Delta^{j}(k)\right) .
$$

Da condição de $\Delta^{j}(g \cdot n)=\pi_{j}(g) \Delta^{j}(n) \pi_{j}(g)^{-1}$ e da 2.2 .3 temos que:

$$
L_{1}^{j}(g \cdot n, g \cdot m, g \cdot k)=L^{j}(n, m, k)
$$

para $g \in S U(2)$, o que implica que o produto twisted é equivariante:

$$
\left(f \star_{1} h\right)^{g}=f^{g} \star_{1} h^{g}
$$

para todo $g \in S U(2)$.

A fórmula acima nos diz que o trikernel é uma função rotacionalmente invariante nas suas entradas vetoriais. De acordo com o teorema de Weyl [22] dependerá apenas da combinação de permutação de produtos escalares e de determinante. Por exemplo, para $j=1 / 2$ tem-se

$$
L_{1}^{1 / 2}(n, m, k)=\frac{1}{4}(1+3(<n, m>+<m, k>+<k, n>)+3 \sqrt{3} i[n, m, k]) .
$$

A partir dessa idéia, procuramos encontrar uma fórmula geral, que representasse o trikernel de Stratonovich (e de Berezin, depois). Fórmula essa que dependeria de produtos internos e determinantes, como Weyl afirmou.

Formalizando o contexto acima, temos a seguinte definição para correspondência de símbolos em geral:

Definição 2.2.1. Para uma dada correspondência de símbolo

$$
W^{j}: B\left(H_{j}\right) \simeq M_{\mathbb{C}}(n+1) \rightarrow \operatorname{Poly}_{\mathbb{C}}\left(S^{2}\right) \subset C_{\mathbb{C}}^{\infty}\left(S^{2}\right),
$$

que associa cada operador $P$ ao símbolo $W_{P}^{j}$, o produto twisted $\star$ de simbolos é a operação binária sobre simbolos

$$
\star: W^{j}\left(\mathcal{B}\left(\mathcal{H}_{j}\right)\right) \times W^{j}\left(\mathcal{B}\left(\mathcal{H}_{j}\right)\right) \rightarrow W^{j}\left(\mathcal{B}\left(\mathcal{H}_{j}\right)\right) \simeq \operatorname{Poly} \mathbb{C}\left(S^{2}\right)
$$

induzida pelo produto de operadores dada por

$$
W_{P Q}^{j}=W_{P}^{j} \star W_{Q}^{j} .
$$


Sejam $g \in S O(3)$ e $f_{1}, f_{2}, f_{3} \in W^{j}\left(B\left(H_{j}\right)\right) \simeq \operatorname{Poly}_{C}\left(S^{2}\right) \subset C_{C}^{\infty}\left(S^{2}\right)$, temos que a algebra de símbolos definida por um qualquer produto twisted satisfaz as seguintes propriedades:

(i) $S O(3)$ - equivariante: $\left(f_{1} \star f_{2}\right)^{g}=f_{1}^{g} \star f_{2}^{g}$;

(ii) associativa: $\left(f_{1} \star f_{2}\right) \star f_{3}=f_{1} \star\left(f_{2} \star f_{3}\right)$;

(iii) unital: $1 \star f=f \star 1=f$;

(iv) estrela algebra: $\overline{f_{1} \star f_{2}}=\overline{f_{2}} \star \overline{f_{1}}$.

Em vista da definição 2.2.1, podemos usar o postulado de normalização da definição de correspondência de símbolos para definir um produto interno induzido no espaço de símbolos:

$$
<W_{P}^{j}, W_{Q}^{j}>_{\star}:=\frac{1}{4 \pi} \int_{S^{2}} \overline{W_{P}^{j}} \star W_{Q}^{j} d S .
$$

Com isso podemos escrever o postulado de isometria (tracionalidade) como

$$
\text { (v) Isometria: } \left.<W_{P}^{j}, W_{Q}^{j}>=<W_{P}^{j}, W_{Q}^{j}\right\rangle_{\star} \text {. }
$$

De fato, por um lado temos

$$
<W_{P}^{j}, W_{Q}^{j}>=<P, Q>_{j}=\frac{1}{n+1} \operatorname{Tr}\left(P^{*} Q\right)
$$

por outro lado,

$$
\begin{aligned}
<W_{P}^{j}, W_{Q}^{j} & >_{\star}=\frac{1}{4 \pi} \int_{S^{2}} \overline{W_{P}^{j}} \star W_{Q}^{j} d S=\frac{1}{4 \pi} \int_{S^{2}} W_{P^{*}}^{j} \star W_{Q}^{j} d S \\
& =\frac{1}{4 \pi} \int_{S^{2}} W_{P^{*} Q}^{j} d S=\frac{1}{n+1} \operatorname{Tr}\left(P^{*} Q\right) .
\end{aligned}
$$

Definição 2.2.2. O produto twisted obtido via correspondência de Stratonovich-Weyl é chamado de produto twisted padrão de símbolos e é denotado por $\star_{1}^{n}$ ou simplismente por $\star_{1}$.

Portanto, para $f, g \in W_{1}^{j}\left(\mathcal{B}\left(\mathcal{H}_{j}\right)\right)=W^{j}\left(\mathcal{B}\left(\mathcal{H}_{j}\right)\right) \simeq \operatorname{Polyc}_{\mathcal{C}}\left(S^{2}\right)_{\leq n} \subset C_{\mathbb{C}}^{\infty}\left(S^{2}\right)$

$\star_{1}=\star_{1}^{n}:(f, g) \mapsto f \star_{1}^{n} g \in W^{j}\left(\mathcal{B}\left(\mathcal{H}_{j}\right)\right) \simeq \operatorname{Pol} y_{\mathcal{C}}\left(S^{2}\right)_{\leq n} \subset C_{\mathbb{C}}^{\infty}\left(S^{2}\right)$.

E finalmente, antes de enfim, introduzir o trikernel de Stratonovich, apresentaremos a representação integral do produto twisted. 
O produto twisted de símbolos esféricos pode ser escrito na forma:

$$
f \star g(n)=\int_{S^{2}} \int_{S^{2}} f\left(n_{1}\right) g\left(n_{2}\right) L\left(n_{1}, n_{2}, n\right) d n_{1} d n_{2} .
$$

A forma integral para o produto twisted nos permite uma definição direta e geral de produto twisted de simbolos arbitrários $f, g \in \operatorname{Pol} y_{C}\left(S^{2}\right)_{\leq n}$ sem ter que decompô-los na base de harmônicos esféricos.

Em tal representação integral todas as propriedades do produto twisted são encontrados no trikernel integral

$$
L: S^{2} \times S^{2} \times S^{2} \rightarrow \mathbb{C}
$$

Nos casos estudados nessa tese, temos que $L=L_{1}^{j}$ ou $L_{\vec{b}}^{j}$.

Para a correspondência de símbolo de Stratonovich-Weyl, $W_{1}^{j}$, segue:

Definição 2.2.3. Sejam $f, g \in \mathcal{C}_{\mathbb{C}}^{\infty}\left(S^{2}\right)$ tais que $f=W_{1}^{j}(F), g=W_{1}^{j}(G)$, para $F, G \in \mathcal{B}\left(\mathcal{H}_{j}\right)$, onde $W_{1}^{j}$ é determinado pelo operador kernel $K_{1}^{j}$ com todos os números característicos $c_{l}=1$, para $0 \leq l \leq n=2 j$, na equação $K=\frac{1}{n+1} I+\sum_{l=1}^{n} c_{l} \sqrt{\frac{2 l+1}{n+1}} e(l, 0)$. $\log$,

$$
f *_{1}^{n} g(n)=\int_{S^{2} \times S^{2}} f\left(n_{1}\right) g\left(n_{2}\right) \mathbb{L}_{1}^{j}\left(n_{1}, n_{2}, n\right) d n_{1} d n_{2},
$$

onde

$$
\mathbb{L}_{1}^{j}\left(n_{1}, n_{2}, n\right)=\left(\frac{2 j+1}{4 \pi}\right)^{2} \operatorname{trace}\left(K_{1}^{j}\left(n_{1}\right) K_{1}^{j}\left(n_{2}\right) K_{1}^{j}(n)\right)
$$

é o trikernel de Stratonovich.

O Trikernel de Stratonovich também pode ser reescrito da seguinte maneira:

$$
\begin{gathered}
\mathbb{L}_{1}^{j}\left(n_{1}, n_{2}, n\right)=\sum_{l_{k}, m_{k}}(-1)^{2 j} \sqrt{(2 j+1)\left(2 l_{1}+1\right)\left(2 l_{2}+1\right)(2 l+1)} . \\
{\left[\begin{array}{ccc}
l_{1} & l_{2} & l \\
m_{1} & m_{2} & -m
\end{array}\right][j] \overline{Y_{l_{1}}^{m_{1}}}\left(n_{1}\right) \overline{Y_{l_{2}}^{m_{2}}}\left(n_{2}\right) \overline{Y_{l}^{m}}(n)}
\end{gathered}
$$

onde a soma em $l_{k}$ e $m_{k}$ satisfazem : $0 \leq l_{k} \leq n=2 j,-l_{k} \leq m_{k} \leq l_{k}$, $\Delta\left(l_{1}, l_{2}, l_{3}\right)=1, m_{1}+m_{2}+m_{3}=0 \mathrm{e}$

$$
\left[\begin{array}{ccc}
l_{1} & l_{2} & l \\
m_{1} & m_{2} & -m
\end{array}\right][j]=\left\{\begin{array}{ccc}
l_{1} & l_{2} & l \\
j & j & j
\end{array}\right\}\left(\begin{array}{ccc}
l_{1} & l_{2} & l \\
-m_{1} & -m_{2} & m
\end{array}\right),
$$

o produto do $6 j$-símbolo pelo $3 j$-símbolo, respectivamente. 
Corolário 2.2.4. O Trikernel de Stratonovich é simétrico, no seguinte sentido:

$$
\mathbb{L}_{1}^{j}\left(n_{1}, n_{2}, n_{3}\right)=\mathbb{L}_{1}^{j}\left(n_{3}, n_{1}, n_{2}\right)=\mathbb{L}_{1}^{j}\left(n_{2}, n_{3}, n_{1}\right) .
$$

Demonstração: Essa simetria é obtida graças a simetria de $\left[\begin{array}{ccc}l_{1} & l_{2} & l \\ m_{1} & m_{2} & -m\end{array}\right][j]$.

Propriedades 2.2.5. Propriedades do Trikernel de Stratonovich.

(a) $L_{1}^{j}\left(n_{1}, n_{2}, n\right)=L_{1}^{j}\left(g n_{1}, g n_{2}, g n\right), \quad g \in S U(2)$

De fato,

$$
\begin{gathered}
L_{1}^{j}\left(g n_{1}, g n_{2}, g n\right)=\left(\frac{2 j+1}{4 \pi}\right)^{2} \operatorname{Tr}\left(K_{1}^{j}\left(g n_{1}\right) K_{1}^{j}\left(g n_{2}\right) K_{1}^{j}(g n)\right) \\
=\left(\frac{2 j+1}{4 \pi}\right)^{2} \operatorname{Tr}\left(\left(\pi_{j}(g) K_{1}^{j}\left(n_{1}\right) \pi_{j}^{-1}(g)\right)\left(\pi_{j}(g) K_{1}^{j}\left(n_{2}\right) \pi_{j}^{-1}(g)\right)\left(\pi_{j}(g) K_{1}^{j}(n) \pi_{j}^{-1}(g)\right)\right) \\
=\left(\frac{2 j+1}{4 \pi}\right)^{2} \operatorname{Tr}\left(K_{1}^{j}\left(n_{1}\right) K_{1}^{j}\left(n_{2}\right) K_{1}^{j}(n)\right) \\
=L_{1}^{j}\left(n_{1}, n_{2}, n\right) .
\end{gathered}
$$

(b) $\int_{S^{2}} L_{1}^{j}\left(n_{1}, n_{2}, n\right) L_{1}^{j}\left(n, n_{3}, n_{4}\right) d n=\int_{S^{2}} L_{1}^{j}\left(n_{1}, n, n_{4}\right) L_{1}^{j}\left(n_{2}, n_{3}, n\right) d n$.

De fato, sejam $f, g, h \in \operatorname{Poly}_{\mathbb{C}}\left(S^{2}\right)$, temos que o produto twisted é associativo, ou seja, $(f \star g) \star h=f \star(g \star h)$, logo, por um lado,

$$
\begin{gathered}
(f \star g) \star h\left(n_{4}\right)=\int_{S^{2}} \int_{S^{2}}(f \star g)(n) h\left(n_{3}\right) L_{1}^{j}\left(n, n_{3}, n_{4}\right) d n d n_{3} \\
=\int_{S^{2}} \int_{S^{2}}\left(\int_{S^{2}} \int_{S^{2}} f\left(n_{1}\right) g\left(n_{2}\right) L_{1}^{j}\left(n_{1}, n_{2}, n\right) d n_{1} d n_{2}\right) h\left(n_{3}\right) L_{1}^{j}\left(n, n_{3}, n_{4}\right) d n d n_{3} \\
\int_{S^{2}} \int_{S^{2}} L_{1}^{j}\left(n_{1}, n_{2}, n\right) \int_{S^{2}} \int_{S^{2}} L_{1}^{j}\left(n, n_{3}, n_{4}\right) f\left(n_{1}\right) g\left(n_{2}\right) h\left(n_{3}\right) d n_{1} d n_{2} d n d n_{3}
\end{gathered}
$$

e por outro,

$$
\begin{gathered}
f \star(g \star h)\left(n_{4}\right)=\int_{S^{2}} \int_{S^{2}} f\left(n_{1}\right)(g \star h)(n) L_{1}^{j}\left(n_{1}, n, n_{4}\right) d n_{1} d n \\
=\int_{S^{2}} \int_{S^{2}} f\left(n_{1}\right)\left(\int_{S^{2}} \int_{S^{2}} g\left(n_{2}\right) h\left(n_{3}\right) L_{1}^{j}\left(n_{2}, n_{3}, n\right) d n_{2} d n_{3}\right) L_{1}^{j}\left(n_{1}, n, n_{4}\right) d n_{1} d n
\end{gathered}
$$




$$
=\int_{S^{2}} \int_{S^{2}} L_{1}^{j}\left(n_{2}, n_{3}, n\right) \int_{S^{2}} \int_{S^{2}} L_{1}^{j}\left(n_{1}, n, n_{4}\right) f\left(n_{1}\right) g\left(n_{2}\right) h\left(n_{3}\right) d n_{2} d n_{3} d n_{1} d n
$$

(c) $\int_{S^{2}} L_{1}^{j}\left(n_{1}, n_{2}, n\right) d n_{1}=R^{j}\left(n_{2}, n\right), \int_{S^{2}} L_{1}^{j}\left(n_{1}, n_{2}, n\right) d n_{2}=R^{j}\left(n_{1}, n\right)$.

onde $R^{j}\left(n_{2}, n\right) \in \operatorname{Poly}_{\mathbb{C}}\left(S^{2}\right)$ é o kernel de reprodução da algebra polinomial truncada $P o l y_{\mathbb{C}}\left(S^{2}\right)$, caracterizado por $\int_{S^{2}} R^{j}\left(n_{2}, n\right) f\left(n_{2}\right) d n_{2}=f(n)$, para toda $f \in \operatorname{Poly}_{\mathbb{C}}\left(S^{2}\right)$.

De fato essa afirmação segue de:

$$
f(n)=(f \star 1)(n)=\int_{S^{2}} \int_{S^{2}} f\left(n_{1}\right) L_{1}^{j}\left(n_{1}, n_{2}, n\right) d n_{1} d n_{2}
$$

$\mathrm{e}$

$$
f(n)=(1 \star f)(n)=\int_{S^{2}} \int_{S^{2}} f\left(n_{2}\right) L_{1}^{j}\left(n_{1}, n_{2}, n\right) d n_{1} d n_{2} .
$$

(d) $L_{1}^{j}\left(n_{2}, n_{1}, n\right)=\overline{L_{1}^{j}\left(n_{1}, n_{2}, n\right)}$.

De fato, como $\overline{L_{1}^{j}\left(n_{1}, n_{2}, n\right)}=\left(\frac{2 j+1}{4 \pi}\right)^{2} \overline{\operatorname{Tr}\left(K_{1}^{j}\left(n_{1}\right) K_{1}^{j}\left(n_{2}\right) K_{1}^{j}(n)\right)}$

$=\left(\frac{2 j+1}{4 \pi}\right)^{2} \operatorname{Tr}\left(\left(K_{1}^{j}\left(n_{1}\right) K_{1}^{j}\right)^{*}\left(n_{2}\right) K_{1}^{j}(n)\right)=\left(\frac{2 j+1}{4 \pi}\right)^{2} \operatorname{Tr}\left(K_{1}^{j}\left(n_{2}\right) K_{1}^{j}\left(n_{1}\right) K_{1}^{j}(n)\right)$

$=L_{1}^{j}\left(n_{2}, n_{1}, n\right)$.

Observamos que para definir o trikernel de Stratonovich e explicitar suas propriedades, começamos com o símbolo de Stratonovich-Weyl $W_{1}^{j}$. Faremos o mesmo agora para definir o trikernel de Berezin.

Seja $B$ a correspondência de símbolo de Berezin padrão. Como essa correspondência de símbolo não é simétrica, existem dois produtos internos naturais $S U(2)$-invariante sobre o espaço de símbolos $\operatorname{Poly}_{\mathbb{C}}^{m}\left(S^{2}\right)$ :

- o produto usual $L^{2}$ sobre $C_{\mathbb{C}}^{\infty}\left(S^{2}\right) \supset \operatorname{Poly}_{\mathbb{C}}\left(S^{2}\right)$ :

$$
<f_{1}, f_{2}>=\frac{1}{4 \pi} \int_{S^{2}} \overline{f_{1}} f_{2} d S=\frac{1}{4 \pi} \int_{S^{2}} \overline{f_{1}}(n) f_{2}(n) d n .
$$

- o produto interno induzido sobre $\operatorname{Poly}_{\mathbb{C}}\left(S^{2}\right) \subset C_{\mathbb{C}}^{\infty}\left(S^{2}\right)$ :

$$
<f_{1}, f_{2}>_{\star \frac{n}{b}}=\frac{1}{4 \pi} f_{1} \star \frac{n}{b} f_{2} d S=\frac{1}{4 \pi} \int_{S^{2}} f_{1}(n) \star \frac{n}{b} f_{2}(n) d n .
$$

Podemos agora definir um trikernel integral, baseando-se nesses dois produtos internos em Poly $y_{\mathbb{C}}\left(S^{2}\right)$, esse trikernel chama-se trikernel de Berezin $L_{\vec{b}}^{j}$, definido por: 


$$
f_{1} \star \frac{n}{b} f_{2}(n)=\iint_{S^{2} \times S^{2}} f_{1}\left(n_{1}\right) f_{2}\left(n_{2}\right) L_{\vec{b}}^{j}\left(n_{1}, n_{2}, n\right) d n_{1} d n_{2} .
$$

Definição 2.2.6. Definimos o trikernel de Berezim, adicionando na fórmula de Stratonovich os números característicos da correspondencia de símbolos padrão de Berezin,

$$
\sqrt{\frac{\left(\begin{array}{c}
n+l_{1}+1 \\
l_{1}
\end{array}\right)\left(\begin{array}{c}
n+l_{2}+1 \\
l_{2}
\end{array}\right)\left(\begin{array}{l}
n \\
l_{3}
\end{array}\right)}{\left(\begin{array}{l}
n \\
l_{1}
\end{array}\right)\left(\begin{array}{l}
n \\
l_{2}
\end{array}\right)\left(\begin{array}{c}
n+l_{3}+1 \\
l_{3}
\end{array}\right)}}
$$

Obtemos:

$$
\begin{aligned}
& L_{\vec{b}}^{j}\left(n_{1}, n_{2}, n\right)=\sum_{l_{k}, m_{k}} \frac{(-1)^{n} \sqrt{(2 j+1)\left(2 l_{1}+1\right)\left(2 l_{2}+1\right)(2 l+1)}}{(4 \pi)^{2}} \\
& {\left[\begin{array}{ccc}
l_{1} & l_{2} & l \\
m_{1} & m_{2} & -m
\end{array}\right][j] \sqrt{\frac{\left(\begin{array}{c}
n+l_{1}+1 \\
l_{1}
\end{array}\right)\left(\begin{array}{c}
n+l_{2}+1 \\
l_{2}
\end{array}\right)\left(\begin{array}{c}
n \\
l_{3}
\end{array}\right)}{\left(\begin{array}{c}
n \\
l_{1}
\end{array}\right)\left(\begin{array}{l}
n \\
l_{2}
\end{array}\right)\left(\begin{array}{c}
n+l_{3}+1 \\
l_{3}
\end{array}\right)}\left(n_{1}\right) \overline{Y_{l_{2}}^{m_{2}}}\left(n_{2}\right) \overline{Y_{l}^{m}}(n)} }
\end{aligned}
$$

onde

$$
\left[\begin{array}{ccc}
l_{1} & l_{2} & l \\
m_{1} & m_{2} & -m
\end{array}\right][j]=\left\{\begin{array}{ccc}
l_{1} & l_{2} & l \\
j & j & j
\end{array}\right\}\left(\begin{array}{ccc}
l_{1} & l_{2} & l \\
-m_{1} & -m_{2} & m
\end{array}\right) .
$$

Observamos que já o Trikernel de Berezin, $L_{\vec{b}}^{j}$ não possui a propriedade de simetrica como o Trikernel de Stratonovich, mas ele satisfaz todas as propriedades 2.2.5 enunciadas para o trikernel de Stratonovich.

\subsection{Cálculos explícitos para o trikernel de Stratonovich e de Berezin.}

Objetivo dessa seção é encontrar fórmulas explícitas para o trikernel de Stratonovich e de Berezin. Para tal, vamos primeiramente obter uma fórmula alternativa que dependa apenas de produtos internos e determinantes que são funções $S O(3)$-invariantes. E o que nos permite encontrar essa nova formulação é o seguinte teorema cuja demonstração se encontra em [22]. 
Teorema 2.3.1. Toda função de três pontos na esfera $S^{2}$, SO(3)-invariante, representada por vetores unitários $n_{1}, n_{2}, n$, no espaço tri dimensional euclideano, pode ser expressada como uma função de três produtos internos

$$
<n_{1}, n_{2}>,<n_{1}, n_{3}>,<n_{2}, n>
$$

junto com o determinante

$$
\left[n_{1}, n_{2}, n_{3}\right]=\operatorname{det}\left(n_{1}, n_{2}, n\right)
$$

Essa fórmula alternativa está explicitada no seguinte:

Teorema 2.3.2. Sejam $n_{1}, n_{2}, n_{0} \in S^{2}, 0 \leq l_{k} \leq 2 j$ e cada tripla $\left(l_{1}, l_{2}, l_{3}\right)$ satisfazendo a propriedade triangular $e-l_{k} \leq m_{k} \leq l_{k}$ para $k=1,2,3$. E seja

$$
\begin{gathered}
A=A\left(n_{1}, n_{2}, n_{0}\right)=<n_{1}, n_{2}>-<n_{0}, n_{1}><n_{0}, n_{2}>, \\
B=B\left(n_{1}, n_{2}, n_{0}\right)=\operatorname{det}\left[n_{1}, n_{2}, n_{0}\right]
\end{gathered}
$$

denotamos:

$$
\begin{gathered}
\mathcal{L}_{l_{1}, l_{2}, l_{3}}\left(n_{1} n_{2}, n_{0}\right)=\sqrt{\left(2 l_{1}+1\right)\left(2 l_{2}+1\right)\left(2 l_{3}+1\right)}\left(\left(\begin{array}{ccc}
l_{1} & l_{2} & l_{3} \\
0 & 0 & 0
\end{array}\right) P_{l_{1}}^{0}\left(z_{1}\right) P_{l_{2}}^{0}\left(z_{2}\right)\right. \\
+\sum_{m=1}^{m i n\left(l_{1}, l_{2}\right)}\left(\begin{array}{ccc}
l_{1} & l_{2} & l_{3} \\
m & -m & 0
\end{array}\right) \sqrt{\frac{\left(l_{1}-m\right) !\left(l_{2}-m\right) !}{\left(l_{1}+m\right) !\left(l_{2}+m\right) !}} P_{l_{1}}^{m}\left(z_{1}\right) P_{l_{2}}^{m}\left(z_{2}\right) \\
\left.(-1)^{m}\left[(A-i B)^{m}+(-1)^{l_{1}+l_{2}+l_{3}}(A+i B)^{m}\right]\right) .
\end{gathered}
$$

Então,

$$
\mathbb{L}_{1}^{j}\left(n_{1}, n_{2}, n_{0}\right)=\frac{(-1)^{2 j} \sqrt{2 j+1}}{(4 \pi)^{2}} \sum_{l_{k}}\left\{\begin{array}{ccc}
l_{1} & l_{2} & l_{3} \\
j & j & j
\end{array}\right\} \mathcal{L}_{l_{1}, l_{2}, l_{3}}
$$

Demonstração: Como o trikernel de Stratonovich é invariante por rotação, usaremos o ponto inicial $n_{1}=\left(x_{1}, y_{1}, z_{1}\right), n_{2}=\left(x_{2}, y_{2}, z_{2}\right)$ e $n_{0}=(0,0,1)$. E depois, generalizaremos para qualquer ponto da esfera, usando que qualquer ponto da esfera pode ser obtido por uma rotação de $n_{0}$. 
Iniciaremos a demonstração com o somatório em $-l_{k} \leq m_{k} \leq l_{k}$ :

$$
\sum_{m_{k}}\left(\begin{array}{ccc}
l_{1} & l_{2} & l_{3} \\
-m_{1} & -m_{2} & m_{3}
\end{array}\right) \cdot \overline{Y_{l_{1}}^{m_{1}}}\left(n_{1}\right) \cdot \overline{Y_{l_{2}}^{-m_{1}}}\left(n_{2}\right) \cdot \overline{Y_{l_{3}}^{m_{3}}}\left(n_{0}\right)
$$

Usaremos que:

1) $Y_{l}^{0}(0, \phi)=\sqrt{2 l+1}$

2) $m_{1}+m_{2}=0^{3}$;

3) $Y_{l}^{m}(\varphi, \theta)=(-1)^{m} \sqrt{\frac{2 l+1}{4 \pi} \cdot \frac{(l-m) !}{(l+m) !}} P_{l}^{m}(\cos \varphi) e^{i m \theta}$;

4) Usando as coordenadas esféricas: $x=\cos (\theta) \operatorname{sen}(\varphi), y=\operatorname{sen}(\theta) \operatorname{sen}(\varphi)$ e $z=$ $\cos (\varphi)$, obtemos que

$$
e^{i m \theta}=\frac{(x+i y)^{m}}{\left(1-z^{2}\right)^{m / 2}}
$$

pois $x+i y=\operatorname{sen}(\varphi)(\cos (\theta)+i \operatorname{sen}(\theta))=\left(1-z^{2}\right)^{1 / 2} e^{i \theta}$.

Segue:

$$
\begin{aligned}
& \sum_{m_{k}}\left(\begin{array}{ccc}
l_{1} & l_{2} & l_{3} \\
-m_{1} & m_{1} & 0
\end{array}\right) \cdot \overline{Y_{l_{1}}^{m_{1}}} \cdot \overline{Y_{l_{2}}^{-m_{1}}} \sqrt{\left(2 l_{3}+1\right)} \\
& =\sum_{\max \left(-l_{1},-l_{2}\right) \leq m_{1} \leq \min \left(l_{1}, l_{2}\right)} \sqrt{2 l_{3}+1}\left(\begin{array}{ccc}
l_{1} & l_{2} & l_{3} \\
-m_{1} & m_{1} & 0
\end{array}\right) \cdot \overline{Y_{l_{1}}^{m_{1}}} \cdot \overline{Y_{l_{2}}^{-m_{1}}} \\
& =\sqrt{2 l_{3}+1}\left(\begin{array}{ccc}
l_{1} & l_{2} & l_{3} \\
0 & 0 & 0
\end{array}\right) \cdot \overline{Y_{l_{1}}^{0}}\left(n_{1}\right) \cdot \overline{Y_{l_{2}}^{0}}\left(n_{2}\right)+ \\
& \sum_{m_{1}=\max \left(-l_{1},-l_{2}\right)}^{-1} \sqrt{2 l_{3}+1}\left(\begin{array}{ccc}
l_{1} & l_{2} & l_{3} \\
-m_{1} & m_{1} & 0
\end{array}\right) \cdot \overline{Y_{l_{1}}^{m_{1}}}\left(n_{1}\right) \cdot \overline{Y_{l_{2}}^{-m_{1}}}\left(n_{2}\right)+ \\
& \sum_{m_{1}=1}^{\min \left(l_{1}, l_{2}\right)} \sqrt{2 l_{3}+1}\left(\begin{array}{ccc}
l_{1} & l_{2} & l_{3} \\
-m_{1} & m_{1} & 0
\end{array}\right) \cdot \overline{Y_{l_{1}}^{m_{1}}}\left(n_{1}\right) \cdot \overline{Y_{l_{2}}^{-m_{1}}}\left(n_{2}\right) \\
& =\sqrt{\left(2 l_{1}+1\right)\left(2 l_{2}+1\right)\left(2 l_{3}+1\right)}\left(\begin{array}{ccc}
l_{1} & l_{2} & l_{3} \\
0 & 0 & 0
\end{array}\right) P_{l_{1}}^{0}\left(z_{1}\right) P_{l_{2}}^{0}\left(z_{2}\right)+ \\
& \sum_{m=1}^{-\max \left(-l_{1},-l_{2}\right)} \sqrt{2 l_{3}+1}\left(\begin{array}{ccc}
l_{1} & l_{2} & l_{3} \\
m & -m & 0
\end{array}\right) \cdot \overline{Y_{l_{1}}^{-m}}\left(n_{1}\right) \cdot \overline{Y_{l_{2}}^{m}}\left(n_{2}\right)+
\end{aligned}
$$

${ }^{3}$ tomamos $m_{3}=0$, pois $n_{0}=(0,0,1)$ implica $(0, \theta)$. 


$$
\begin{aligned}
& \sum_{m=1}^{\min \left(l_{1}, l_{2}\right)} \sqrt{2 l_{3}+1}\left(\begin{array}{ccc}
l_{1} & l_{2} & l_{3} \\
-m_{1} & m_{1} & 0
\end{array}\right) \cdot \overline{Y_{l_{1}}^{m_{1}}}\left(n_{1}\right) \cdot \overline{Y_{l_{2}}^{-m_{1}}}\left(n_{2}\right) \\
= & \sqrt{\left(2 l_{1}+1\right)\left(2 l_{2}+1\right)\left(2 l_{3}+1\right)}\left(\begin{array}{ccc}
l_{1} & l_{2} & l_{3} \\
0 & 0 & 0
\end{array}\right) P_{l_{1}}^{0}\left(z_{1}\right) P_{l_{2}}^{0}\left(z_{2}\right)+
\end{aligned}
$$

$$
\begin{aligned}
& \sum_{m=1}^{\min \left(l_{1}, l_{2}\right)} \sqrt{2 l_{3}+1}(-1)^{m}\left(\begin{array}{ccc}
l_{1} & l_{2} & l_{3} \\
m & -m & 0
\end{array}\right)\left\{Y_{l_{1}}^{m}\left(n_{1}\right) \cdot \overline{Y_{l_{2}}^{m}}\left(n_{2}\right)+(-1)^{l_{1}+l_{2}+l_{3}} \overline{Y_{l_{1}}^{m}}\left(n_{1}\right) \cdot Y_{l_{2}}^{m}\left(n_{2}\right)\right\} \\
& =\sqrt{\left(2 l_{1}+1\right)\left(2 l_{2}+1\right)\left(2 l_{3}+1\right)}\left(\begin{array}{ccc}
l_{1} & l_{2} & l_{3} \\
0 & 0 & 0
\end{array}\right) P_{l_{1}}^{0}\left(z_{1}\right) P_{l_{2}}^{0}\left(z_{2}\right)+ \\
& \sum_{m=1}^{\min \left(l_{1}, l_{2}\right)} \sqrt{2 l_{3}+1}(-1)^{m}\left(\begin{array}{ccc}
l_{1} & l_{2} & l_{3} \\
m & -m & 0
\end{array}\right) . \\
& \left\{\sqrt{2 l_{1}+1} \sqrt{\frac{\left(l_{1}-m\right) !}{\left(l_{1}+m\right) !}} P_{l_{1}}^{m}\left(z_{1}\right) \frac{\left(x_{1}+i y_{1}\right)^{m}}{\left(1-z_{1}^{2}\right)^{m / 2}} \sqrt{2 l_{2}+1} \sqrt{\frac{\left(l_{2}-m\right) !}{\left(l_{2}+m\right) !}} P_{l_{2}}^{m}\left(z_{2}\right) \frac{\left(x_{2}-i y_{2}\right)^{m}}{\left(1-z_{2}^{2}\right)^{m / 2}}+\right. \\
& \left.(-1)^{l_{1}+l_{2}+l_{3}} \sqrt{2 l_{1}+1} \sqrt{\frac{\left(l_{1}-m\right) !}{\left(l_{1}+m\right) !}} P_{l_{1}}^{m}\left(z_{1}\right) \frac{\left(x_{1}-i y_{1}\right)^{m}}{\left(1-z_{1}^{2}\right)^{m / 2}} \sqrt{2 l_{2}+1} \sqrt{\frac{\left(l_{2}-m\right) !}{\left(l_{2}+m\right) !}} P_{l_{2}}^{m}\left(z_{2}\right) \frac{\left(x_{2}+i y_{2}\right)^{m}}{\left(1-z_{2}^{2}\right)^{m / 2}}\right\} \\
& =\sqrt{\left(2 l_{1}+1\right)\left(2 l_{2}+1\right)\left(2 l_{3}+1\right)}\left(\begin{array}{ccc}
l_{1} & l_{2} & l_{3} \\
0 & 0 & 0
\end{array}\right) P_{l_{1}}^{0}\left(z_{1}\right) P_{l_{2}}^{0}\left(z_{2}\right)+ \\
& +\sum_{m=1}^{\min \left(l_{1}, l_{2}\right)} \sqrt{\left(2 l_{1}+1\right)\left(2 l_{2}+1\right)\left(2 l_{3}+1\right)}(-1)^{m} \sqrt{\frac{\left(l_{1}-m\right) !\left(l_{2}-m\right) !}{\left(l_{1}+m\right) !\left(l_{2}+m\right) !}} \\
& \frac{P_{l_{1}}^{m}\left(z_{1}\right) P_{l_{2}}^{m}\left(z_{2}\right)}{\left(1-z_{1}^{2}\right)^{m / 2}\left(1-z_{2}^{2}\right)^{m / 2}}\left(\begin{array}{ccc}
l_{1} & l_{2} & l_{3} \\
m & -m & 0
\end{array}\right) \text {. } \\
& \left\{\left(x_{1}+i y_{1}\right)^{m_{1}}\left(x_{2}+i y_{2}\right)^{m_{1}}+(-1)^{l_{1}+l_{2}+l_{3}}\left(x_{1}-i y_{1}\right)^{m_{1}}\left(x_{2}+i y_{2}\right)^{m_{1}}\right\}
\end{aligned}
$$

Analisando essa ultima parcela separadamente, obtemos:

$$
\left\{\left(\left(x_{1}+i y_{1}\right)\left(x_{2}+i y_{2}\right)\right)^{m}+(-1)^{l_{1}+l_{2}+l_{3}}\left(\left(x_{1}-i y_{1}\right)\left(x_{2}+i y_{2}\right)\right)^{m}\right\}
$$




$$
=\left\{\left(x_{1} x_{2}+y_{1} y_{2}-i\left(x_{1} y_{2}-x_{2} y_{1}\right)\right)^{m}+(-1)^{l_{1}+l_{2}+l_{3}}\left(x_{1} x_{2}+y_{1} y_{2}+i\left(x_{1} y_{2}-x_{2} y_{1}\right)\right)^{m}\right\},
$$

E assim, denotando por

$$
\begin{gathered}
A=A\left(n_{1}, n_{2}, n_{0}\right) \\
=<n_{1}, n_{2}>-<n_{0}, n_{1}><n_{0}, n_{2}> \\
=x_{1} x_{2}+y_{1} y_{2}
\end{gathered}
$$

e

$$
\begin{gathered}
B=B\left(n_{1}, n_{2}, n_{0}\right)=\operatorname{det}\left[n_{1}, n_{2}, n_{0}\right] \\
=x_{1} y_{2}-x_{2} y_{1} .
\end{gathered}
$$

Finalmente, concluimos que o somatório em $m_{k}$ fica da seguinte maneira:

$$
\begin{gathered}
\sqrt{\left(2 l_{1}+1\right)\left(2 l_{2}+1\right)\left(2 l_{3}+1\right)}\left(\begin{array}{ccc}
l_{1} & l_{2} & l_{3} \\
0 & 0 & 0
\end{array}\right) P_{l_{1}}^{0}\left(z_{1}\right) P_{l_{2}}^{0}\left(z_{2}\right) \\
+\sqrt{\left(2 l_{1}+1\right)\left(2 l_{2}+1\right)\left(2 l_{3}+1\right)} \sum_{m=1}^{m i n\left(l_{1}, l_{2}\right)}(-1)^{m} \sqrt{\frac{\left(l_{1}-m\right) !\left(l_{2}-m\right) !}{\left(l_{1}+m\right) !\left(l_{2}+m\right) !}} \\
\frac{P_{l_{1}}^{m}\left(z_{1}\right) P_{l_{2}}^{m}\left(z_{2}\right)}{\left(1-z_{1}^{2}\right)^{m / 2}\left(1-z_{2}^{2}\right)^{m / 2}}\left(\begin{array}{ccc}
l_{1} & l_{2} & l_{3} \\
m & -m & 0
\end{array}\right)\left[(A-i B)^{m}+(-1)^{l_{1}+l_{2}+l_{3}}(A+i B)^{m}\right]
\end{gathered}
$$

Uma observação desse trikernel, é que podemos abrir a soma $\mathcal{L}_{l_{1}, l_{2}, l_{3}}\left(n_{1}, n_{2}, n_{0}\right)$ em duas partes, aquela em que $l_{1}+l_{2}+l_{3}=$ par e aquela em que

$$
\left(\begin{array}{ccc}
l_{1} & l_{2} & l_{3} \\
0 & 0 & 0
\end{array}\right)=0
$$

se $l_{1}+l_{2}+l_{3}=$ mpar.

Segue:

$$
\begin{gathered}
\bullet l_{1}+l_{2}+l_{3}=\text { par } \\
\mathcal{L}_{l_{1}, l_{2}, l_{3}}\left(n_{1} n_{2}, n_{0}\right)=\sqrt{\left(2 l_{1}+1\right)\left(2 l_{2}+1\right)\left(2 l_{3}+1\right)}\left(\left(\begin{array}{ccc}
l_{1} & l_{2} & l_{3} \\
0 & 0 & 0
\end{array}\right) P_{l_{1}}^{0}\left(z_{1}\right) P_{l_{2}}^{0}\left(z_{2}\right)\right.
\end{gathered}
$$




$$
\begin{gathered}
+\sum_{m=1}^{m i n\left(l_{1}, l_{2}\right)}\left(\begin{array}{ccc}
l_{1} & l_{2} & l_{3} \\
m & -m & 0
\end{array}\right) \sqrt{\frac{\left(l_{1}-m\right) !\left(l_{2}-m\right) !}{\left(l_{1}+m\right) !\left(l_{2}+m\right) !}} P_{l_{1}}^{m}\left(z_{1}\right) P_{l_{2}}^{m}\left(z_{2}\right) \\
\left.2(-1)^{m}\left[\sum_{k=0}^{m}(-1)^{k}\left(\begin{array}{c}
m \\
2 k
\end{array}\right) A^{m-2 k} B^{2 k}\right]\right) . \\
\bullet l_{1}+l_{2}+l_{3}=m p a r \\
\mathcal{L}_{l_{1}, l_{2}, l_{3}\left(n 1 n_{2}, n_{0}\right)=} 2 i \sqrt{\left(2 l_{1}+1\right)\left(2 l_{2}+1\right)\left(2 l_{3}+1\right)} \sum_{m=1}^{m i n\left(l_{1}, l_{2}\right)}\left(\begin{array}{ccc}
l_{1} & l_{2} & l_{3} \\
m & -m & 0
\end{array}\right) \\
\sqrt{\frac{\left(l_{1}-m\right) !\left(l_{2}-m\right) !}{\left(l_{1}+m\right) !\left(l_{2}+m\right) !} P_{l_{1}}^{m}\left(z_{1}\right) P_{l_{2}}^{m}\left(z_{2}\right)} \\
(-1)^{m}\left[\sum_{k=0}^{m}(-1)^{k+1}\left(\begin{array}{c}
m \\
2 k+1
\end{array}\right) A^{m-(2 k+1)} B^{2 k+1}\right]
\end{gathered}
$$

Observamos que é na soma ímpar que obtemos a parte imagínária.

Analogamente o Trikernel de Berezin ficará com a seguinte formulação:

Teorema 2.3.3. Sejam $n_{1}, n_{2}, n_{0} \in S^{2}, 0 \leq l_{k} \leq 2 j$ e cada tripla $\left(l_{1}, l_{2}, l_{3}\right)$ satisfazendo a propriedade triangular $e-l_{k} \leq m_{k} \leq l_{k}$ para $k=1,2,3$. E seja

$$
\begin{gathered}
A=A\left(n_{1}, n_{2}, n_{0}\right)=<n_{1}, n_{2}>-<n_{0}, n_{1}><n_{0}, n_{2}>, \\
B=B\left(n_{1}, n_{2}, n_{0}\right)=\operatorname{det}\left[n_{1}, n_{2}, n_{0}\right]
\end{gathered}
$$

denotamos:

$$
\begin{gathered}
\mathcal{L}_{l_{1}, l_{2}, l_{3}}\left(n_{1} n_{2}, n_{0}\right)=\sqrt{\left(2 l_{1}+1\right)\left(2 l_{2}+1\right)\left(2 l_{3}+1\right)}\left(\left(\begin{array}{ccc}
l_{1} & l_{2} & l_{3} \\
0 & 0 & 0
\end{array}\right) P_{l_{1}}^{0}\left(z_{1}\right) P_{l_{2}}^{0}\left(z_{2}\right)\right. \\
+\sum_{m=1}^{m i n\left(l_{1}, l_{2}\right)}\left(\begin{array}{ccc}
l_{1} & l_{2} & l_{3} \\
m & -m & 0
\end{array}\right) \sqrt{\frac{\left(l_{1}-m\right) !\left(l_{2}-m\right) !}{\left(l_{1}+m\right) !\left(l_{2}+m\right) !}} P_{l_{1}}^{m}\left(z_{1}\right) P_{l_{2}}^{m}\left(z_{2}\right) \\
\left.(-1)^{m}\left[(A-i B)^{m}+(-1)^{l_{1}+l_{2}+l_{3}}(A+i B)^{m}\right]\right) .
\end{gathered}
$$


Então, obtemos para o trikernel de Berezin,

$$
\begin{gathered}
\mathbb{L}_{\vec{b}}^{j}\left(n_{1}, n_{2}, n_{0}\right)=\frac{(-1)^{2 j} \sqrt{2 j+1}}{(4 \pi)^{2}} \sum_{l_{k}} \sqrt{\frac{\left(\begin{array}{c}
n+l_{1}+1 \\
l_{1}
\end{array}\right)\left(\begin{array}{c}
n+l_{2}+1 \\
l_{2}
\end{array}\right)\left(\begin{array}{l}
n \\
l_{3}
\end{array}\right)}{\left(\begin{array}{l}
n \\
l_{1}
\end{array}\right)\left(\begin{array}{l}
n \\
l_{2}
\end{array}\right)\left(\begin{array}{c}
n+l_{3}+1 \\
l_{3}
\end{array}\right)}} \\
\left\{\begin{array}{ccc}
l_{1} & l_{2} & l_{3} \\
j & j & j
\end{array}\right\} \mathcal{L}_{l_{1}, l_{2}, l_{3} .}
\end{gathered}
$$

Para generalizar, ambos os resultados, para um ponto $n$ qualquer na esfera $S^{2}$, basta trocarmos $n_{0}$ por $n$.

\subsubsection{Cálculos explícitos para $j=\frac{1}{2}, 1, \frac{3}{2}, 2$.}

Vamos explicitar os exemplos de $j$ sitados nessa subseção. Lembrando que as triplas $\left(l_{1}, l_{2}, l_{3}\right)$ devem satisfazer a desigualdade triangular $\left|l_{1}-l_{2}\right| \leq l_{3} \leq l_{1}+l_{2}$. E ainda,

$$
A=<n_{1}, n_{2}>-<n_{0}, n_{1}><n_{0}, n_{2}>
$$

e

$$
B=\operatorname{det}\left(n_{1}, n_{2}, n_{0}\right)=\left[n_{1}, n_{2}, n_{0}\right] .
$$

$\bullet j=1 / 2$ Como $0 \leq l_{k} \leq 2 j=1$,

temos as seguintes triplas $(0,0,0),(0,1,1),(1,0,1),(1,1,1)$.

$$
\begin{gathered}
\mathcal{L}_{0,0,0}\left(n_{1}, n_{2}, n_{0}\right)=1 ; \\
\mathcal{L}_{1,1,0}\left(n_{1}, n_{2}, n_{0}\right)=-\sqrt{3}<n_{1}, n_{2}>;
\end{gathered}
$$

a partir desse obtemos $\mathcal{L}_{1,0,1}\left(n_{1}, n_{2}, n_{0}\right)$ e $\mathcal{L}_{0,1,1}\left(n_{1}, n_{2}, n_{0}\right)$, usando ciclicidade.

$$
\mathcal{L}_{1,1,1}\left(n_{1}, n_{2}, n_{0}\right)=\frac{3}{\sqrt{2}} i B
$$

Agora somando em $l_{k}$,

$$
L_{1}^{1 / 2}\left(n_{1}, n_{2}, n_{0}\right)=\frac{1}{(4 \pi)^{2}}\left\{1+3\left(<n_{1}, n_{2}>+<n_{1}, n_{0}>+<n_{2}, n_{0}>\right)+i 3 \sqrt{3}\left[n_{1}, n_{2}, n_{0}\right]\right\}
$$


$\left.L_{\vec{b}}^{1 / 2}\left(n_{1}, n_{2}, n_{0}\right)=\frac{1}{(4 \pi)^{2}}\left\{1+3<n_{1}, n_{2}>+<n_{1}, n_{0}>+<n_{2}, n_{0}>\right)+i \sqrt{3}\left[n_{1}, n_{2}, n_{3}\right]\right\}$

$\bullet j=1$ Como $0 \leq l_{k} \leq 2 j$, $\log l_{k} \in\{0,1,2\}$.

Teremos as seguintes triplas: $(0,0,0),(0,1,1),(1,0,1),(1,1,0),(1,1,1),(1,1,2)$, $(1,2,1),(1,2,2),(0,2,2),(2,0,2),(2,1,1),(2,1,2),(2,2,0),(2,2,1),(2,2,2)$. Primeiramente faremos o cálculo de $\mathcal{L}_{l_{1}, l_{2}, l_{3}}\left(n_{1}, n_{2}, n_{0}\right)$, para depois calcular o operador.

$$
\begin{gathered}
\mathcal{L}_{0,0,0}\left(n_{1}, n_{2}, n_{0}\right)=1 ; \\
\mathcal{L}_{1,1,0}\left(n_{1}, n_{2}, n_{0}\right)=-\sqrt{3}<n_{1}, n_{2}>; \\
\mathcal{L}_{1,1,1}\left(n_{1}, n_{2}, n_{0}\right)=\frac{3}{\sqrt{2}} i B ; \\
\mathcal{L}_{1,1,2}\left(n_{1}, n_{2}, n_{0}\right)=\frac{3 \sqrt{2}}{2 \sqrt{3}}\left(3<n_{0}, n_{1}><n_{0}, n_{2}>-<n_{1}, n_{2}>\right) ; \\
\mathcal{L}_{1,2,2}\left(n_{1}, n_{2}, n_{0}\right)=-\frac{15 \sqrt{2}}{2 \sqrt{5}} i B<n_{0}, n_{1}>; \\
\mathcal{L}_{0,2,2}\left(n_{1}, n_{2}, n_{0}\right)=\frac{\sqrt{5}}{2}\left(3<n_{0}, n_{2}>^{2}-1\right) ; \\
\mathcal{L}_{2,2,2}\left(n_{1}, n_{2}, n_{0}\right)=-\frac{5 \sqrt{2}}{4 \sqrt{7}}\left(9 B^{2}+3 R-5\right) .
\end{gathered}
$$

Somando em $l_{k}$ na soma do trikernel de Stratonovich obtemos:

$$
\begin{gathered}
L_{1}^{1}\left(n_{1}, n_{2}, n_{0}\right)=\frac{1}{(4 \pi)^{2}}\left\{1+3 P+\frac{15}{2}(R-1)+\frac{9 \sqrt{2}}{4} i B(1+5 P)\right. \\
\left.+\frac{3 \sqrt{10}}{8}\left(3 P^{2}-3 R-2 P\right)-\frac{5 \sqrt{10}}{8}\left(9 B^{2}-5+3 R\right)\right\} .
\end{gathered}
$$

E para Berezin: 


$$
\begin{gathered}
L_{\vec{b}}^{1}\left(n_{1}, n_{2}, n_{0}\right)=\frac{1}{(4 \pi)^{2}}\left(1+\frac{11 \sqrt{5}}{4 \sqrt{3}}-\frac{6}{\sqrt{3}}+P\right. \\
<n_{1}, n_{2}>+\frac{3}{2 \sqrt{5}}<n_{0}, n_{1}><n_{0}, n_{2}>+\frac{3 \sqrt{2}}{2}<n_{1}, n_{2}><n_{0}, n_{1}> \\
-\frac{1}{2 \sqrt{15}}<n_{1}, n_{2}>-\frac{\sqrt{2}}{2 \sqrt{3}}<n_{0}, n_{1}>-\frac{\sqrt{2}}{2 \sqrt{3}}<n_{0}, n_{2}> \\
+\frac{3}{2} i B P+6 i B<n_{0}, n_{2}>+\frac{3}{2 \sqrt{3}}\left(<n_{0}, n_{2}>^{2}+<n_{0}, n_{1}>^{2}\right) \\
\left.+\frac{15}{\sqrt{3}}<n_{1}, n_{2}>^{2}-\frac{15 \sqrt{5}}{4 \sqrt{3}} B^{2}-\frac{9 \sqrt{5}}{4 \sqrt{3}} R\right) .
\end{gathered}
$$

Onde, para facilitar a notação e o excesso, denotamos:

$$
\begin{gathered}
P=<n_{0}, n_{1}>+<n_{0}, n_{2}>+<n_{1}, n_{2}> \\
R=<n_{0}, n_{1}>^{2}+<n_{0}, n_{2}>^{2}+<n_{1}, n_{2}>^{2} .
\end{gathered}
$$

$\bullet j=3 / 2$ Como $0 \leq l_{k} \leq 2 j$, logo $l_{k} \in\{0,1,2,3\}$.

Teremos as seguintes triplas: $(0,0,0),(0,1,1),(1,0,1),(1,1,0),(1,1,1),(1,1,2)$, $(1,2,1),(1,2,2),(0,2,2),(2,0,2),(2,1,1),(2,1,2),(2,2,0),(2,2,1),(2,2,2),(0,3,3)$, $(1,2,3),(1,3,2),(1,3,3),(2,1,3),(2,2,3),(2,3,1),(2,3,2),(2,3,3),(3,0,3),(3,1,2)$, $(3,1,3),(3,2,1),(3,2,2),(3,2,3),(3,3,0),(3,3,1),(3,3,2),(3,3,3)$.

Observe que além das triplas que apareceram no caso $j=1$, apareceram algumas a mais. Logo, ao calcular os $\mathcal{L}_{l_{1}, l_{2}, l_{3}}$, para esse caso, basta calcular para essas triplas a mais.

$$
\mathcal{L}_{0,3,3}\left(n_{1}, n_{2}, n_{0}\right)=-\frac{7}{2 \sqrt{7}}\left(5<n_{0}, n_{2}>^{3}-3<n_{0}, n_{2}>\right) ;
$$

por ciclicidade achamos $\mathcal{L}_{3,0,3}\left(n_{1}, n_{2}, n_{0}\right)$ e $\mathcal{L}_{3,3,0}\left(n_{1}, n_{2}, n_{0}\right)$.

$$
\mathcal{L}_{1,3,3}\left(n_{1}, n_{2}, n_{0}\right)=\frac{-21}{4 \sqrt{7}} i B\left(1-5<n_{0}, n_{2}>^{2}\right)
$$

por ciclicidade achamos $\mathcal{L}_{3,1,3}\left(n_{1}, n_{2}, n_{0}\right)$ e $\mathcal{L}_{3,3,1}\left(n_{1}, n_{2}, n_{0}\right)$. 
$\mathcal{L}_{1,2,3}\left(n_{1}, n_{2}, n_{0}\right)=-\frac{15}{2}<n_{0}, n_{1}><n_{0}, n_{2}>^{2}+\frac{3}{2}<n_{0}, n_{1}>+3<n_{0}, n_{2}><n_{1}, n_{2}>;$

por ciclicidade obtemos todas as outras combinações $\mathcal{L}_{3,1,2}\left(n_{1}, n_{2}, n_{0}\right), \mathcal{L}_{2,3,1}\left(n_{1}, n_{2}, n_{0}\right)$, $\mathcal{L}_{2,1,3}\left(n_{1}, n_{2}, n_{0}\right), \mathcal{L}_{3,2,1}\left(n_{1}, n_{2}, n_{0}\right)$ e $\mathcal{L}_{1,3,2}\left(n_{1}, n_{2}, n_{0}\right)$.

$$
\mathcal{L}_{2,2,3}\left(n_{1}, n_{2}, n_{0}\right)=15 \sqrt{\frac{2}{5}} i B\left(2<n_{0}, n_{1}><n_{0}, n_{2}>-<n_{1}, n_{2}>\right) ;
$$

por ciclicidade obtemos $\mathcal{L}_{3,2,2}\left(n_{1}, n_{2}, n_{0}\right)$ e $\mathcal{L}_{2,3,2}\left(n_{1}, n_{2}, n_{0}\right)$.

$$
\begin{gathered}
\mathcal{L}_{2,3,3}\left(n_{1}, n_{2}, n_{0}\right)=\frac{7}{4 \sqrt{21}}\left(-10<n_{0}, n_{2}>^{3}-15<n_{0}, n_{1}>^{2}<n_{0}, n_{2}>\right. \\
-15<n_{0}, n_{2}><n_{1}, n_{2}>^{2}+6<n_{0}, n_{2}>-3<n_{0}, n_{1}><n_{1}, n_{2}> \\
\left.+45<n_{0}, n_{1}><n_{0}, n_{2}>^{2}<n_{1}, n_{2}>+15 B^{2}<n_{0}, n_{2}>\right),
\end{gathered}
$$

por ciclicidade obtemos $\mathcal{L}_{3,2,3}\left(n_{1}, n_{2}, n_{0}\right)$ e $\mathcal{L}_{3,3,2}\left(n_{1}, n_{2}, n_{0}\right)$.

$$
\mathcal{L}_{3,3,3}\left(n_{1}, n_{2}, n_{0}\right)=\frac{35}{4 \sqrt{7}}\left(-3 R-5 B^{2}+21 / 5\right) .
$$

Juntando tudo no somatório em $l_{k}$, obtemos:

$$
\begin{gathered}
L_{1}^{3 / 2}\left(n_{0}, n_{1}, n_{2}\right)=\frac{1}{(4 \pi)^{2}}\left\{\frac{5}{2}+\frac{3}{20}\left(P-11 R-25 B^{2} P+P^{2}-15 P R\right)\right. \\
+P^{3}-3 B^{2}+\frac{5}{2} S+\frac{1}{8} \sqrt{\frac{3}{35}}\left(87-90 R-100 B^{2}\right) \\
\left.-\frac{i B}{2 \sqrt{5}}\left(7-6 P-30 P^{2}+30 R-\frac{15}{2} \sqrt{\frac{3}{7}} R\right)\right\} \\
L_{\vec{b}}^{3 / 2}\left(n_{0}, n_{1}, n_{2}\right)=\frac{1}{(4 \pi)^{2}}\left(\frac{5}{2}-P-\frac{2}{3}<n_{1}, n_{2}>-\frac{1}{\sqrt{3}} i B\left(1+6<n_{0}, n_{2}>\right)\right. \\
\frac{3}{\sqrt{3}} i B P+\frac{2}{5} \sqrt{\frac{2}{3}}<n_{1}, n_{2}>-3 \sqrt{\frac{10}{3}}\left(<n_{0}, n_{1}>+<n_{0}, n_{2}>\right) \\
-2 \frac{\sqrt{5}}{5}<n_{0}, n_{1}><n_{0}, n_{2}>+9 \sqrt{\frac{10}{3}}<n_{1}, n_{2}><n_{0}, n_{1}>+<n_{0}, n_{2}><n_{1}, n_{2}>
\end{gathered}
$$




$$
\begin{aligned}
& -6<n_{1}, n_{2}>^{2}-\frac{3}{2} R-\frac{5}{2} S+90<n_{1}, n_{2}>^{3}+\frac{3}{2} P-54<n_{1}, n_{2}> \\
& +\frac{1}{5} \sqrt{\frac{5}{21}}\left(21 P+12<n_{0}, n_{1}>+12<n_{0}, n_{2}>+42<n_{0}, n_{1}><n_{0}, n_{2}>\right. \\
& +63<n_{1}, n_{2}><n_{0}, n_{2}>+63<n_{1}, n_{2}><n_{0}, n_{1}>-\frac{15}{2}<n_{0}, n_{1}><n_{0}, n_{2}>^{2} \\
& -\frac{15}{2}<n_{0}, n_{2}><n_{0}, n_{1}>^{2}-\frac{105}{2}<n_{1}, n_{2}><n_{0}, n_{1}>^{2}-\frac{105}{2}<n_{1}, n_{2}><n_{0}, n_{2}>^{2} \\
& \left.-\frac{315}{2}<n_{0}, n_{2}><n_{1}, n_{2}>^{2}-\frac{315}{2}<n_{0}, n_{1}><n_{1}, n_{2}>^{2}\right) \\
& +\frac{3}{\sqrt{3}} i B\left(22+\frac{1}{\sqrt{5}}-5<n_{0}, n_{2}>^{2}-\frac{5}{\sqrt{5}}<n_{0}, n_{1}>^{2}-15<n_{1}, n_{2}>^{2}\right) \\
& -\frac{6}{\sqrt{7}} i B\left(-7 P+6<n_{1}, n_{2}>+2<n_{0}, n_{1}><n_{0}, n_{2}>\right. \\
& \left.+14<n_{0}, n_{1}><n_{1}, n_{2}>+14<n_{0}, n_{2}><n_{1}, n_{2}>\right) \\
& +\frac{\sqrt{5}}{20}\left(-10 S-15<n_{0}, n_{1}>^{2}<n_{0}, n_{2}>-15<n_{1}, n_{2}>^{2}<n_{0}, n_{1}>\right. \\
& -15<n_{0}, n_{2}><n_{1}, n_{2}>^{2}-15<n_{0}, n_{1}><n_{0}, n_{2}>^{2}+6<n_{0}, n_{2}> \\
& +6<n_{0}, n_{1}>-3<n_{0}, n_{1}><n_{1}, n_{2}>-3<n_{0}, n_{2}><n_{1}, n_{2}> \\
& +45<n_{0}, n_{1}><n_{0}, n_{2}>^{2}<n_{1}, n_{2}>+15 B^{2} P \\
& +45<n_{1}, n_{2}><n_{0}, n_{1}>^{2}<n_{01}, n_{2}> \\
& -60<n_{1}, n_{2}>^{3}+90 B^{2}<n_{1}, n_{2}>7\left(-15<n_{0}, n_{2}>^{2}<n_{1}, n_{2}>\right. \\
& -15<n_{0}, n_{1}>^{2}<n_{1}, n_{2}>+6<n_{1}, n_{2}>-3<n_{0}, n_{2}><n_{1}, n_{0}> \\
& \left.\left.+45<n_{0}, n_{2}><n_{1}, n_{2}>^{2}<n_{0}, n_{1}>\right)\right) \\
& \left.-\frac{5}{4} \sqrt{\frac{3}{2}}\left(-3 R-5 B^{3}+21 / 5\right)\right) \text {. }
\end{aligned}
$$

onde, $P$ e $R$ como no caso $j=1$ e

$$
S=<n_{0}, n_{1}>^{3}+<n_{0}, n_{2}>^{3}+<n_{1}, n_{2}>^{3} .
$$

$\bullet j=2$ Como $0 \leq l_{k} \leq 2 j, \log 0 l_{k} \in\{0,1,2,3,4\}$.

Teremos, além das triplas para $j=3 / 2$, mais essas : $(0,4,4),(4,0,4),(4,4,0)$, $(1,3,4),(4,1,3),(3,4,1),(4,3,1),(1,4,3),(3,1,4),(1,4,4),(4,1,4),(4,4,1)$, 
$(2,4,4),(4,2,4),(4,4,2),(3,4,4),(4,3,4),(4,4,3),(4,4,4),(2,2,4),(4,2,2),(2,4,2)$, $(2,3,4),(4,2,3),(3,4,2),(4,3,2),(2,4,3),(3,2,4),(3,3,4),(4,3,3),(3,4,3)$.

$$
\mathcal{L}_{0,4,4}\left(n_{1}, n_{2}, n_{0}\right)=\frac{3}{8}\left(3-30<n_{0}, n_{2}>^{2}+35<n_{0}, n_{2}>^{4}\right),
$$

por ciclicidade obtemos $\mathcal{L}_{4,0,4}\left(n_{1}, n_{2}, n_{0}\right)$ e $\mathcal{L}_{4,4,0}\left(n_{1}, n_{2}, n_{0}\right)$.

$$
\begin{aligned}
\mathcal{L}_{1,3,4}\left(n_{1}, n_{2}, n_{0}\right) & =\frac{\sqrt{27}}{12}\left(35<n_{0}, n_{1}><n_{0}, n_{2}>^{3}-15<n_{0}, n_{1}><n_{0}, n_{2}>\right. \\
& \left.+3<n_{1}, n_{2}>-15<n_{1}, n_{2}><n_{0}, n_{2}>^{2}\right),
\end{aligned}
$$

por ciclicidade obtemos $\mathcal{L}_{4,1,3}\left(n_{1}, n_{2}, n_{0}\right), \mathcal{L}_{3,4,1}\left(n_{1}, n_{2}, n_{0}\right), \mathcal{L}_{4,3,1}\left(n_{1}, n_{2}, n_{0}\right)$, $\mathcal{L}_{1,4,3}\left(n_{1}, n_{2}, n_{0}\right), \mathcal{L}_{3,1,4}\left(n_{1}, n_{2}, n_{0}\right)$.

$$
\mathcal{L}_{1,4,4}\left(n_{1}, n_{2}, n_{0}\right)=\frac{15}{4} \sqrt{\frac{3}{5}} i B\left(7<n_{0}, n_{2}>^{3}-3<n_{0}, n_{2}>\right),
$$

por ciclicidade obtemos $\mathcal{L}_{4,1,4}\left(n_{1}, n_{2}, n_{0}\right)$ e $\mathcal{L}_{4,4,1}\left(n_{1}, n_{2}, n_{0}\right)$.

$$
\begin{gathered}
\mathcal{L}_{2,4,4}\left(n_{1}, n_{2}, n_{0}\right)=\frac{45}{8 \sqrt{77}}\left(1-10<n_{0}, n_{2}>^{2}-<n_{0}, n_{1}>^{2}+\frac{35}{3}<n_{0}, n_{2}>^{4}\right. \\
+11<n_{0}, n_{1}>^{2}<n_{0}, n_{2}>^{2}+12<n_{0}, n_{1}><n_{0}, n_{2}><n_{1}, n_{2}> \\
-21 B^{2}<n_{0}, n_{2}>^{2}+3 B^{2}+21<n_{1}, n_{2}>^{2}<n_{0}, n_{2}>^{2} \\
\left.-56<n_{0}, n_{1}><n_{0}, n_{2}>^{3}<n_{1}, n_{2}>-3<n_{1}, n_{2}>^{2}\right)
\end{gathered}
$$

por ciclicidade obtemos $\mathcal{L}_{4,2,4}\left(n_{1}, n_{2}, n_{0}\right)$ e $\mathcal{L}_{4,4,2}\left(n_{1}, n_{2}, n_{0}\right)$.

$$
\begin{gathered}
\mathcal{L}_{2,2,4}\left(n_{1}, n_{2}, n_{0}\right)=\frac{5}{8} \sqrt{\frac{18}{35}}\left(2-6<n_{0}, n_{1}>^{2}-6<n_{0}, n_{2}>^{2}+<n_{1}, n_{2}>^{2}\right. \\
\left.-18<n_{0}, n_{1}><n_{0}, n_{2}><n_{1}, n_{2}>+35<n_{0}, n_{1}>^{2}<n_{0}, n_{2}>^{2}-B^{2}\right),
\end{gathered}
$$

por ciclicidade obtemos $\mathcal{L}_{4,2,2}\left(n_{1}, n_{2}, n_{0}\right)$ e $\mathcal{L}_{2,4,2}\left(n_{1}, n_{2}, n_{0}\right)$.

$\mathcal{L}_{2,3,4}\left(n_{1}, n_{2}, n_{0}\right)=\frac{5 \sqrt{9}}{4} i B\left(<n_{0}, n_{1}>-7<n_{0}, n_{1}><n_{0}, n_{2}>^{2}+2<n_{0}, n_{2}><n_{1}, n_{1}>\right)$, 
por ciclicidade obtemos $\mathcal{L}_{4,2,3}\left(n_{1}, n_{2}, n_{0}\right), \mathcal{L}_{3,4,2}\left(n_{1}, n_{2}, n_{0}\right), \mathcal{L}_{4,3,2}\left(n_{1}, n_{2}, n_{0}\right)$, $\mathcal{L}_{2,4,3}\left(n_{1}, n_{2}, n_{0}\right), \mathcal{L}_{3,2,4}\left(n_{1}, n_{2}, n_{0}\right)$

$$
\begin{aligned}
& \mathcal{L}_{3,3,4}\left(n_{1}, n_{2}, n_{0}\right)=\frac{105}{8 \sqrt{154}}\left(-2<n_{0}, n_{1}>^{3}<n_{0}, n_{2}>^{3}+11<n_{0}, n_{1}>^{3}<n_{0}, n_{2}>\right. \\
& +11<n_{0}, n_{1}><n_{0}, n_{2}>^{3}-7<n_{0}, n_{1}><n_{0}, n_{2}>-\frac{1}{5}<n_{1}, n_{2}> \\
& +<n_{0}, n_{1}>^{2}<n_{1}, n_{2}>+<n_{0}, n_{2}>^{2}<n_{1}, n_{2}>-30<n_{0}, n_{1}>^{2}<n_{0}, n_{2}>^{2}<n_{1}, n_{2}> \\
& -11 B^{2}<n_{0}, n_{1}><n_{0}, n_{2}>+11<n_{0}, n_{1}><n_{0}, n_{2}><n_{1}, n_{2}>^{2} \\
& \left.+<n_{1}, n_{2}>^{3}-3 B^{2}<n_{1}, n_{2}>\right),
\end{aligned}
$$

por ciclicidade obtemos $\mathcal{L}_{4,3,3}\left(n_{1}, n_{2}, n_{0}\right)$ e $\mathcal{L}_{3,4,3}\left(n_{1}, n_{2}, n_{0}\right)$.

$$
\begin{gathered}
\mathcal{L}_{3,4,4}\left(n_{1}, n_{2}, n_{0}\right)=\frac{1}{8} \sqrt{\frac{5}{154}} i B\left(-21<n_{0}, n_{2}>^{3}+9<n_{0}, n_{2}>\right. \\
+140<n_{1}, n_{2}><n_{0}, n_{1}><n_{0}, n_{2}>^{2}-10<n_{0}, n_{1}><n_{1}, n_{2}>-35<n_{0}, n_{1}>^{2}<n_{0}, n_{2}> \\
\left.+\frac{35}{3} B^{2}<n_{0}, n_{2}>-35<n_{0}, n_{2}><n_{1}, n_{2}>^{2}\right),
\end{gathered}
$$

por ciclicidade obtemos $\mathcal{L}_{4,3,4}\left(n_{1}, n_{2}, n_{0}\right)$ e $\mathcal{L}_{4,4,3}\left(n_{1}, n_{2}, n_{0}\right)$.

$$
\begin{gathered}
\mathcal{L}_{4,4,4}\left(n_{1}, n_{2}, n_{0}\right)=\frac{5}{96 \sqrt{2002}}\left(\frac{1611}{5}+1715 B^{4}-1950 B^{2}\right. \\
\left.-426 R+1470 R B^{2}+105 R^{2}+42 Q,\right)
\end{gathered}
$$

onde $Q=<n_{0}, n_{1}>^{4}+<n_{0}, n_{2}>^{4}+<n_{1}, n_{2}>^{4}$.

Juntando no somatório em $l_{k}$, obtemos:

$$
\begin{gathered}
L_{1}^{2}\left(n_{1}, n_{2}, n_{0}\right)=\frac{5}{14(4 \pi)^{2}}\left(\frac{12833}{1344}-\frac{3}{4} \sqrt{\frac{7}{2}}-\frac{45 \sqrt{5}}{56}+\frac{15 \sqrt{35}}{4}\right. \\
-\frac{21 \sqrt{3}}{8} i B-\frac{7 \sqrt{7}}{4} i B-\frac{9655}{1008} B^{2}+\frac{249 \sqrt{5}}{56} B^{2}-\frac{15 \sqrt{35}}{4} B^{2}+\frac{25 \sqrt{3}}{8} i B^{3} \\
+\frac{1225}{1728} B^{4}+\frac{35}{4} \sqrt{\frac{7}{2}} M+\frac{25 \sqrt{5}}{8} M+\frac{8517}{112} P-\frac{15}{2} \sqrt{\frac{5}{7}} P-\sqrt{\frac{7}{2}} P-\frac{1361}{\sqrt{5} 28} P \\
+\frac{51}{4 \sqrt{7}} i B P-\frac{495}{16} B^{2} P-\frac{585 \sqrt{5}}{56} B^{2} P-\frac{695}{112} P^{2}-\frac{15}{2} \sqrt{\frac{5}{7}} P^{2}+\frac{579}{56 \sqrt{5}} P^{2}
\end{gathered}
$$




$$
\begin{gathered}
\frac{60}{\sqrt{7}} i B P^{2}-\frac{195}{16} B^{2} P^{2}-\frac{5}{32} P^{3}+\frac{5 \sqrt{5}}{4} P^{3}+\frac{5 \sqrt{35}}{4} P^{3}+\frac{15}{16} B^{2} P^{3}+\frac{35}{4} P^{4}-\frac{15}{64} P^{6} \\
+\frac{5}{288} Q-\frac{3349}{504} R-\frac{75}{4} \sqrt{\frac{5}{7}} R-\frac{9}{2} \sqrt{\frac{7}{2}} R+\frac{741}{28 \sqrt{5}} R+\frac{15 \sqrt{3}}{8} i B R-\frac{135}{4 \sqrt{7}} i B R \\
+\frac{3685}{288} B^{2} R-\frac{105 \sqrt{5}}{8} B^{2} R-\frac{2595}{32} P R \\
+\frac{417 \sqrt{5}}{56} P R+\frac{115}{32} P^{2} R-\frac{30 \sqrt{5}}{7} P^{2} R+\frac{15}{16} P^{3} R+\frac{45}{64} P^{4} R-\frac{3125}{576} R^{2}-\frac{75 \sqrt{5}}{14} R^{2} \\
\left.-\frac{45}{64} P^{2} R^{2}+\frac{15}{64} R^{3}+\frac{5}{4} S+5 \sqrt{\frac{7}{2}} S-\frac{3 \sqrt{5}}{4} S-\frac{5 \sqrt{35}}{4} S-\frac{35 \sqrt{7}}{4} i B S-\frac{15}{16} R S\right) .
\end{gathered}
$$

Reagrupando, obtemos:

$$
\begin{aligned}
& L_{1}^{2}\left(n_{1}, n_{2}, n_{0}\right)=\frac{5}{14(4 \pi)^{2}}\left(\frac{12833}{1344}-\frac{3}{4} \sqrt{\frac{7}{2}}-\frac{45 \sqrt{5}}{56}+\frac{15 \sqrt{35}}{4}\right. \\
& +\left(\frac{35}{4} \sqrt{\frac{7}{2}}+\frac{25 \sqrt{5}}{8}\right) M+\left(\frac{8517}{112}-\frac{15}{2} \sqrt{\frac{5}{7}}-\sqrt{\frac{7}{2}}-\frac{1361}{\sqrt{528}}\right) P \\
& +\frac{5}{288} Q-\left(\frac{3349}{504}+\frac{75}{4} \sqrt{\frac{5}{7}}+\frac{9}{2} \sqrt{\frac{7}{2}}-\frac{741}{28 \sqrt{5}}\right) R \\
& \left(\frac{5}{4}+5 \sqrt{\frac{7}{2}}-\frac{3 \sqrt{5}}{4}-\frac{5 \sqrt{35}}{4}\right) S+\left(\frac{579}{56 \sqrt{5}}-\frac{695}{112}-\frac{15}{2} \sqrt{\frac{5}{7}}\right) P^{2} \\
& +\left(\frac{249 \sqrt{5}}{56}-\frac{9655}{1008}-\frac{15 \sqrt{35}}{4}\right) B^{2}+\left(\frac{417 \sqrt{5}}{56}-\frac{2595}{32}\right) P R \\
& -\left(\frac{3125}{576}+\frac{75 \sqrt{5}}{14}\right) R^{2}-\frac{15}{16} R S \\
& +\left(\frac{5 \sqrt{35}}{4}-\frac{5}{32}+\frac{5 \sqrt{5}}{4}\right) P^{3}-\left(\frac{495}{16}+\frac{585 \sqrt{5}}{56}\right) B^{2} P \\
& +\frac{15}{64} R^{3}+\left(\frac{3685}{288}-\frac{105 \sqrt{5}}{8}\right) B^{2} R+\left(\frac{115}{32}-\frac{30 \sqrt{5}}{7}\right) P^{2} R \\
& +\frac{1225}{1728} B^{4}-\frac{195}{16} B^{2} P^{2}+\frac{15}{16} B^{2} P^{3}+\frac{35}{4} P^{4}+\frac{15}{16} P^{3} R-\frac{15}{64} P^{6}-\frac{45}{64} P^{2} R^{2}+\frac{45}{64} P^{4} R \\
& \left.+i\left\{\left(-\frac{21 \sqrt{3}}{8}-\frac{7 \sqrt{7}}{4}\right) B+\frac{51}{4 \sqrt{7}} B P+\left(\frac{15 \sqrt{3}}{8}-\frac{135}{4 \sqrt{7}}\right) B R-\frac{35 \sqrt{7}}{4} B S+\frac{25 \sqrt{3}}{8} B^{3}+\frac{60}{\sqrt{7}} B P^{2}\right\}\right) .
\end{aligned}
$$

E finalmente para o caso de Berezin, mas como o produto de Berezin não é tão agrupável quanto o de Stratonovich, vamos admitir uma notação para cada 
produto interno, da seguinte maneira:

$$
P_{i, j}=<n_{i}, n_{j}>
$$

E assim:

$$
\begin{aligned}
& L_{\vec{b}}^{2}=\frac{1}{(4 \pi)^{2}} \cdot\left(\frac{47585}{8}+1215 \sqrt{\frac{5}{14}}+\frac{145387}{1344 \sqrt{14}}-\frac{21 \sqrt{21}}{4}-\left(294-\frac{9 \sqrt{3}}{4}+\frac{1}{24 \sqrt{14}}+\frac{793}{4 \sqrt{70}}\right) P_{1,2}\right. \\
& -\left(\frac{476133}{8}+\frac{15551}{48 \sqrt{14}}+\frac{6945}{4} \sqrt{\frac{35}{2}}\right) P_{1,2}^{2}+\left(490+\frac{5}{24} \sqrt{\frac{7}{2}}+2 \sqrt{70}\right) P_{1,2}^{3}+\left(\frac{138915}{2}+2025 \sqrt{\frac{35}{2}}\right) P_{1,2}^{4} \\
& +\left(-\frac{3}{2}+\sqrt{3}+\frac{1737}{2 \sqrt{14}}-\frac{101}{2 \sqrt{70}}\right) P_{0,1}+\left(-\frac{10575}{2 \sqrt{14}}-\frac{3}{2 \sqrt{70}}+\frac{165}{8} \sqrt{\frac{5}{14}}-\frac{4905}{8} \sqrt{\frac{7}{2}}\right) P_{1,2}^{2} P_{0,1} \\
& +\frac{13245}{8} \sqrt{\frac{7}{2}} P_{1,2}^{3} P_{0,1}-\left(\frac{9}{4}+\frac{1081}{8 \sqrt{14}}+\frac{8355}{16} \sqrt{\frac{35}{2}}\right) P_{0,1}^{2} \\
& +\left(\frac{5}{6 \sqrt{14}}+7 \sqrt{70}\right) P_{1,2} P_{0,1}^{2}+\left(\frac{408405}{16} \sqrt{\frac{5}{14}}+135 \sqrt{\frac{7}{2}}\right) P_{1,2}^{2} P_{0,1}^{2} \\
& +\left(\frac{5}{2}+\frac{135}{8} \sqrt{\frac{7}{2}}+\frac{1}{4} \sqrt{\frac{35}{2}}\right) P_{0,1}^{3}+\frac{405}{2} \sqrt{7 / 2} P_{1,2} P_{0,1}^{3}-\frac{135}{4} \sqrt{\frac{7}{2}} P_{1,2}^{3} P_{0,1}^{3}+\left(\frac{35}{8}+\frac{25}{16} \sqrt{35 / 2}\right) P_{0,1}^{4} \\
& +\left(\frac{25 \sqrt{21}}{4}-\frac{110531}{2016 \sqrt{14}}+261 \sqrt{70}\right) B^{2}-\frac{5}{8} \sqrt{\frac{7}{2}} B^{2} P_{1,2}-3645 \sqrt{\frac{35}{2}} B^{2} P_{1,2}^{2} \\
& +\left(\frac{15}{4} \sqrt{\frac{5}{14}}+\frac{135}{2 \sqrt{14}}\right) P_{0,1}^{2} P_{0,2}+\frac{1485}{8} \sqrt{\frac{7}{2}} P_{1,2} P_{0,1}^{2} P_{0,2}-\frac{2025}{4} \sqrt{\frac{7}{2}} P_{1,2}^{2} P_{0,1}^{2} P_{0,2} \\
& +\left(\frac{29205}{2} \sqrt{\frac{7}{2}} B^{2} P_{0,1}-\frac{1485}{8} \sqrt{\frac{7}{2}} B_{1,2}^{2} P_{1,2} P_{0,1}^{3} P_{0,1}-\frac{45}{16} \sqrt{\frac{35}{2}} B^{2} P_{0,1}^{2}\right. \\
& +\frac{175}{1728} \sqrt{\frac{7}{2}} B^{4}+\left(\sqrt{3}-\frac{3}{2}-\frac{7}{4} \sqrt{\frac{7}{10}}+\frac{1737}{2 \sqrt{14}}\right) P_{0,2} \\
& \left.+\frac{3}{2} \sqrt{70}\right) P_{1,2} P_{0,2}
\end{aligned}
$$




$$
\begin{aligned}
& -\frac{1485}{8} \sqrt{72} B^{2} P_{1,2} P_{0,2}-\frac{55}{24} \sqrt{72} B^{2} P_{0,1} P_{0,2}- \\
& -\left(\frac{9}{4}+\frac{1081}{8 \sqrt{14}}+\frac{2805}{16} \sqrt{\frac{35}{2}}\right) P_{0,2}^{2}-\left(\frac{1195}{24 \sqrt{14}}-10 \sqrt{70}\right) P_{1,2} P_{0,2}^{2} \\
& +\left(\frac{214155}{16} \sqrt{\frac{5}{14}}+135 \sqrt{\frac{7}{2}}\right) P_{1,2}^{2} P_{0,2}^{2}+\left(\frac{9}{8} \sqrt{\frac{5}{14}}+\frac{135}{8} \sqrt{7 / 2}\right) P_{0,1} P_{0,2}^{2} \\
& +\frac{1485}{8} \sqrt{7 / 2} P_{1,2} P_{0,1} P_{0,2}^{2}-\frac{2025}{4} \sqrt{7 / 2} P_{1,2}^{2} P_{0,1} P_{0,2}^{2}+\left(15 \sqrt{10 / 7}+\frac{5}{48} \sqrt{7 / 2}\right) P_{0,1}^{2} P_{0,2}^{2} \\
& -\frac{25}{4} \sqrt{7 / 2} P_{1,2} P_{0,1}^{2} P_{0,2}^{2}-\frac{45}{16} \sqrt{35 / 2} B^{2} P_{0,2}^{2}+\left(\frac{5}{2}+135 / 8 \sqrt{7 / 2}+\frac{1}{4} \sqrt{\frac{5}{2}}\right) P_{0,2}^{3} \\
& +\frac{405}{2} \sqrt{\frac{7}{2}} P_{1,2} P_{0,2}^{3}-\frac{135}{4} \sqrt{\frac{7}{2}} P_{1,2}^{3} P_{0,2}^{3} \\
& +\frac{5}{2} \sqrt{\frac{7}{2}} P_{0,1} P_{0,2}^{3}-15 / 2 \sqrt{\frac{35}{2}} P_{1,2} P_{0,1} P_{0,2}^{3}-\frac{5}{12} \sqrt{\frac{7}{2}} P_{0,1}^{3} P_{0,2}^{3} \\
& +\left(\frac{35}{8}+\frac{25}{16} \sqrt{35 / 2}\right) P_{0,2}^{4} \\
& +\frac{5}{288 \sqrt{14}} Q+\left(\frac{9}{8} \sqrt{5 / 14}-\frac{355}{2016 \sqrt{14}}+\frac{15}{4} \sqrt{21}\right) R+\frac{25}{288} \sqrt{\frac{7}{2}} B^{2} R+\frac{25}{576 \sqrt{14}} R^{2} \\
& +i B\left\{-\frac{205 \sqrt{2}}{2}+\left(\frac{158809}{4 \sqrt{2}}-\frac{2295}{8} \sqrt{\frac{5}{2}}-\frac{1215}{8 \sqrt{14}}\right) P_{1,2}+245 \sqrt{2} P_{1,2}^{2}+\left(\frac{405}{8} \sqrt{\frac{7}{2}}-46305 \sqrt{2}\right) P_{1,2}^{3}\right. \\
& -\left(\frac{321}{8 \sqrt{2}}+\frac{5}{12} \sqrt{\frac{5}{2}}+\frac{15}{8 \sqrt{14}}\right) P_{0,1}+\left(\frac{25}{6 \sqrt{14}}+48 \sqrt{2}-\frac{2295}{4} \sqrt{\frac{5}{2}}\right) P_{1,2} P_{0,1} \\
& +\left(\frac{35}{24} \sqrt{\frac{5}{2}}+\frac{25}{24} \sqrt{\frac{7}{2}}\right) P_{1,2}^{2} P_{0,1}+\frac{45}{8 \sqrt{2}} P_{0,1}^{2}+\left(\frac{945}{8} \sqrt{\frac{5}{2}}+\frac{675}{8} \sqrt{\frac{7}{2}}\right) P_{1,2} P_{0,1}^{2} \\
& +\left(\frac{5}{8} \sqrt{\sqrt{7} 2}-\frac{105}{8 \sqrt{2}}\right) P_{0,1}^{3}+\left(\frac{5}{8} \sqrt{\frac{7}{2}}-\frac{105}{8 \sqrt{2}}\right) P_{0,2}^{3}+\frac{45}{8 \sqrt{2}} P_{0,2}^{2} \\
& -\frac{225}{8} \sqrt{\frac{7}{2}} B^{2} P_{1,2}-\frac{25}{72} \sqrt{\frac{7}{2}} B^{2} P_{0,1}-\frac{25}{6} \sqrt{7 / 2} P_{1,2} P_{0,1} P_{0,2}^{2} \\
& +\left(\frac{675}{8} \sqrt{7 / 2}+945 \sqrt{10}\right) P_{1,2} P_{0,2}^{2}+\left(\frac{35}{24} \sqrt{5 / 2}+\frac{25}{24} \sqrt{7 / 2}\right) P_{0,1} P_{0,2}^{2} \\
& -\left(\frac{321}{8 \sqrt{2}}+\frac{2295}{8} \sqrt{\frac{5}{2}}+\frac{15}{8 \sqrt{14}}\right) P_{0,2}+\left(48 \sqrt{2}-\frac{5}{6} \sqrt{\frac{5}{2}}\right) P_{1,2} P_{0,2} \\
& +\left(\frac{25}{24} \sqrt{7 / 2}+945 \sqrt{10}\right) P_{1,2}^{2} P_{0,2}+\left(3 \sqrt{2}-\frac{2295}{4} \sqrt{5 / 2}+\frac{675}{4 \sqrt{14}}\right) P_{0,1} P_{0,2}
\end{aligned}
$$




$$
\left.-\frac{675}{2} \sqrt{7 / 2} P_{1,2}^{2} P_{0,1} P_{0,2}+\left(\frac{945}{8} \sqrt{5 / 2}+\frac{25}{24} \sqrt{7 / 2}\right) P_{0,1}^{2} P_{0,2}-\frac{25}{6} \sqrt{\frac{7}{2}} P_{1,2} P_{0,1}^{2} P_{0,2}-\frac{25}{72} \sqrt{72} B^{2} P_{0,2}\right\}
$$





\begin{tabular}{|c|c|}
\hline & Capítulo \\
\hline 3 \\
\hline
\end{tabular}

\section{Exploração preliminar das assintóticas} para os produtos de Stratonovich de Berezin de funções fortemente oscilatórias

A transição da física clássica para a física quantica é realizada por meio da quantização levando em conta os efeitos causados pelo aparecimento da constante de Planck. Por sua vez o limite semi clássico é um fenômeno físico caracterizado pela dequantização, na qual, a constante de Planck também desempenha um papel importante. Matematicamente podemos representar tal situação física através do procedimento tomando o limite

$$
\hbar \rightarrow 0
$$

embora fisicamente $\hbar$ é uma constante fixada por natureza.

- Como devemos descrever a física básica durante o domínio de transição

$$
\text { quântica } \rightarrow \text { clássica? }
$$

Elucidar esse ponto continua sendo um delicado problema uma vez que envolve a investigação do processo de quantização e dequantização. Não iremos nos aprofundar 
nesse assunto de forma geral. O que queremos, nesse capítulo, é justamente um limite semi clássico, mas somente para sistemas $j$ - spin, que é o contexto dessa tese. Estudar esse limite, nesse contexto, é estudar o limite de $n=2 j \rightarrow \infty$ ( nesse caso $\hbar$ pode ser omitida). Em outras palavras, queremos explorar o que acontecerá quando, nos produtos de Stratonovich e de Berezin, $j \rightarrow \infty$.

Em [12] foi feita uma análise assintótica desses produtos e a dinâmica em primeira ordem é dada pelo parênteses de Poisson. Essa analise foi feita tomando $l$ fixo e $j$ muito grande. O que se concluiu foi que para inteiros não negativos $l_{1}, l_{2}$, o produto twisted padrão (Stratonovich padrão e de Berezin padrão) de esféricos harmônicos $Y_{l_{1}}^{m_{1}}$ e $Y_{l_{2}}^{m_{2}}$ satisfaz:

$$
\begin{gathered}
(i) \quad \lim _{n \rightarrow \infty}\left(Y_{l_{1}}^{m_{1}} \star_{1}^{n} Y_{l_{2}}^{m_{2}}-Y_{l_{2}}^{m_{2}} \star_{1}^{n} Y_{l_{1}}^{m_{1}}\right)=0 \\
\text { (ii) } \lim _{n \rightarrow \infty}\left(Y_{l_{1}}^{m_{1}} \star_{1}^{n} Y_{l_{2}}^{m_{2}}+Y_{l_{2}}^{m_{2}} \star_{1}^{n} Y_{l_{1}}^{m_{1}}\right)=2 Y_{l_{1}}^{m_{1}} Y_{l_{2}}^{m_{2}} ; \\
\text { (iii) } \lim _{n \rightarrow \infty}\left(n\left[Y_{l_{1}}^{m_{1}} \star_{1}^{n} Y_{l_{2}}^{m_{2}}-Y_{l_{2}}^{m_{2}} \star_{1}^{n} Y_{l_{1}}^{m_{1}}\right]\right)=2 i\left\{Y_{l_{1}}^{m_{1}}, Y_{l_{2}}^{m_{2}}\right\} ; \\
\text { (iv) } \lim _{n \rightarrow \infty}\left(Y_{l_{1}}^{m_{1}} \star_{1}^{n} Y_{l_{2}}^{m_{2}}+Y_{l_{2}}^{m_{2}} \star_{1}^{n} Y_{l_{1}}^{m_{1}}-2 Y_{l_{1}}^{m_{1}} Y_{l_{2}}^{m_{2}}\right)=0 ;
\end{gathered}
$$

A partir disso, definiu-se que uma correspondência de símbolos pode ser de vários tipos, incluindo os tipos Poisson ou anti-Poisson (que por sua vez inclui os tipos puro Poisson e anti-puro Poisson) dependendo de quais propriedades acima são satisfeitas.

Mas genericamente, correspondência de símbolos não são do tipo Poisson e nem anti-Poisson. Isso pode ser visto até mesmo no caso restrito da correspondência de símbolo de Stratonovich-Weyl, considerando uma sequência aleatória dada pelos números característicos $c_{l}^{n}=\varepsilon_{l}^{n}= \pm 1$, para $1 \leq l \leq n$ (maior detalhes ver [12]).

Porém, tanto a correspondência de Stratonovich padrão e a de Berezin padrão são do tipo Poisson.

A assintótica considerada em [12] pode ser chamada de limite semi clássico para funções fracamente oscilatórias ( $l$ fínito). O que dizer do limite semi clássico do produto twisted de funções fortemente oscilatórias? Em outras palavras, lembrando que $l \leq 2 j$, podemos ter $l \rightarrow \infty$ quando $j \rightarrow \infty$. Então, como estudar o limite semi clássico de $Y_{l_{1}}^{m_{1}} \star^{j} Y_{l_{2}}^{m_{2}}$ quando $j \rightarrow \infty$ e $l_{1}$ e $l_{2} \rightarrow \infty$ ?

No capítulo anterior, buscamos expressões explícitas para os trikernels de Stratonovich e de Berezin. Esperavamos, de início, obter fórmulas explícitas que nos permi- 
tissem encontrar expressões fechadas para $\mathbb{L}_{1}^{j}$ e $\mathbb{L}_{\vec{b}}^{j}$ no limite $j \rightarrow \infty$. Uma vez tendo estas, poderíamos usar as fórmulas integrais de $\star_{1} \mathrm{e} \star \vec{b}$ para estudar o limite semi clássico destes produtos quando as funções multiplicadas fossem fortemente oscilatórias de maneira similar ao tratamento que é dado ao produto de Weyl [13].

Porém, os cálculos apresentados no capítulo anterior frustraram nossas expectativas, ao menos por hora, pois as expressões para $\mathbb{L}_{1}^{j}$ e $\mathbb{L}_{\vec{b}}^{j}$ ficam mais e mais complicadas, conforme $j$ aumenta e não conseguimos, por meio destas, obter expressões assintóticas para $\mathbb{L}_{1}^{j}$ e $\mathbb{L}_{\vec{b}}^{j}$, quando $j \rightarrow \infty$.

Como alternativa, vamos nesse capítulo, fazer uma exploração preliminar assintótica dos produtos de Stratonovich e de Berezin para harmônicos esféricos, quando $l \rightarrow \infty$ e $j \rightarrow \infty$, mantendo $\frac{l}{j}$ constante e finalmente compará-las.

Observação: dizemos que as funções são fortemente oscilatórias quando $l \rightarrow \infty$.

\subsection{Exploração assintótica dos produtos twisted padrão.}

Vamos apresentar o produto twisted de harmônicos esféricos que originam os produtos de Stratonovich e de Berezin. Seremos breve nessa definição, sem causar nenhum prejuizo de entendimento. Para mais detalhes consultar [12].

Definição 3.1.1. O produto twisted padrão de harmônicos esféricos é dado por

$Y_{l_{1}}^{m_{1}} *_{1} Y_{l_{2}}^{m_{2}}=(-1)^{2 j+m} \sum_{l=0}^{2 j} \sqrt{(2 j+1)\left(2 l_{1}+1\right)\left(2 l_{2}+1\right)(2 l+1)} \cdot\left[\begin{array}{ccc}l_{1} & l_{2} & l \\ m_{1} & m_{2} & -m\end{array}\right][j] Y_{l}^{m}$,

que é justamente o produto de Stratonovich padrão.

O produto twisted induzido pela correspondência de Berezin padrão definida pelos números característicos $b_{l}^{n} \in \mathbb{R}^{+}$dados em 2.1.2 é obtido introduzindo os números característicos de Berezin á expressão acima:

$$
\begin{array}{r}
Y_{l_{1}}^{m_{1}} * \vec{b} Y_{l_{2}}^{m_{2}}=(-1)^{2 j+m} \sum_{l=0}^{2 j} \sqrt{(2 j+1)\left(2 l_{1}+1\right)\left(2 l_{2}+1\right)(2 l+1)}, \\
{\left[\begin{array}{ccc}
l_{1} & l_{2} & l \\
m_{1} & m_{2} & -m
\end{array}\right][j] \frac{b_{l}^{n}}{b_{l_{1}}^{n} b_{l_{2}}^{n}} Y_{l}^{m},}
\end{array}
$$

onde 


$$
\frac{b_{l}^{n}}{b_{l_{1}}^{n} b_{l_{2}}^{n}}=\sqrt{\frac{\left(\begin{array}{c}
n+l_{1}+1 \\
l_{1}
\end{array}\right)\left(\begin{array}{c}
n+l_{2}+1 \\
l_{2}
\end{array}\right)\left(\begin{array}{l}
n \\
l
\end{array}\right)}{\left(\begin{array}{l}
n \\
l_{1}
\end{array}\right)\left(\begin{array}{l}
n \\
l_{2}
\end{array}\right)\left(\begin{array}{c}
n+l+1 \\
l
\end{array}\right)}}
$$

e em ambas as expressões acimas definimos também,

$$
\left[\begin{array}{ccc}
l_{1} & l_{2} & l \\
m_{1} & m_{2} & -m
\end{array}\right][j]=\left\{\begin{array}{ccc}
l_{1} & l_{2} & l \\
j & j & j
\end{array}\right\} \cdot\left(\begin{array}{ccc}
l_{1} & l_{2} & l \\
-m_{1} & -m_{2} & m
\end{array}\right)
$$

de tal maneira que $\left|l_{1}-l_{2}\right| \leq l \leq l_{1}+l_{2}, l_{1}+l_{2}+l \in \mathbb{Z}$ e $m_{1}+m_{2}=m$.

O que faremos a partir dessa definição é tentar encontrar expressões assintóticas para os $3 j, 6 j$ símbolos e para $Y_{l}^{m}$. Colocá-las nos produtos acima e comparar seus resultados.

Vale enfatizar que neste tipo de assintótica a ser desenvolvida, sempre mantemos $l=\lambda j$ e $m=j \mu$, com $\lambda$ e $\mu$ constantes.

Começamos pelo artigo do Ponzano-Regge [17] onde foi dada a seguinte fórmula assintótica para o $6 j$-símbolo, quando todos os números são proporcionais a $j$ e $j \rightarrow \infty$ :

$$
\left\{\begin{array}{lll}
a & b & c \\
d & e & f
\end{array}\right\} \sim \frac{1}{\sqrt{12 \pi V}} \cos \left(\sum_{h, k=1}^{4} j_{h k} \theta_{h k}+\pi / 4\right),
$$

onde $\theta_{h k}=\theta_{k h}(k \neq h=1,2,3,4)$ são os ângulos entre as normais exteriores das duas faces que pertencem a $\operatorname{aresta}^{1} j_{h k}$. E V o volume do tetraedro de arestas $j_{j h}$ e vértices $P_{h}, h=1,2,3,4$.. Dado por:

$$
2^{3}(3 !)^{2} \mathbf{V}^{2}=\left|\begin{array}{ccccc}
0 & d^{2} & e^{2} & f^{2} & 1 \\
d^{2} & 0 & c^{2} & b^{2} & 1 \\
e^{2} & c^{2} & 0 & a^{2} & 1 \\
f^{2} & b^{2} & a^{2} & 0 & 1 \\
1 & 1 & 1 & 1 & 0
\end{array}\right|
$$

Seja $A_{h}$ a área da face oposta ao vértice $h$, então temos:

$$
A_{h} A_{k} \operatorname{sen}\left(\theta_{h k}\right)=\frac{3}{2} \mathbf{V} j_{h k}, \quad h \neq k=1,2,3,4 .
$$

E também tem-se a fórmula para o $3 j-$ smbolo:

\footnotetext{
${ }^{1}$ lembramos que adotamos a representação espacial do $6 j$-símbolo como sendo o tetraedro, detalhes ver Apendice B.
} 


$$
\begin{gathered}
\left(\begin{array}{ccc}
a & b & c \\
m_{a} & m_{b} & m_{c}
\end{array}\right) \sim(2 \pi \mathfrak{A})^{\frac{1}{2}}(-1)^{a+b-c} . \\
\sim \cos \left((a+1 / 2) A+(b+1 / 2) B+(c+1 / 2) C-m_{b} D+m_{a} E+\frac{\pi}{4}\right)
\end{gathered}
$$

onde $m_{a}=e-f, m_{b}=f-d, m_{c}=d-e$ são as terceiras componentes de $a, b, c$ ao longo da direção quando $P_{4} \rightarrow \infty$. $\mathfrak{A}$ é a área do triângulo sombreado que corresponde a projeção de $P_{1} P_{2} P_{3}$ no plano perpendicular ao eixo $z^{2}$. Os ângulos $A, B, C, D$ e $E$ são definidos da seguinte forma em termos de $m_{a}, m_{b}$ e $m_{c}$ por:

$$
\begin{gathered}
\cos (A)=\frac{2(a+1 / 2)^{2} m_{c}+m_{a}\left[(c+1 / 2)^{2}+(a+1 / 2)^{2}-(b+1 / 2)^{2}\right]}{\sqrt{\left[(a+1 / 2)^{2}+m_{a}^{2}\right]\left[4(c+1 / 2)(a+1 / 2)-\left((a+1 / 2)^{2}+(a+1 / 2)^{2}-(b+1 / 2)^{2}\right)^{2}\right]}} \\
\cos (D)=\frac{1}{2} \frac{(a+1 / 2)^{2}-(b+1 / 2)^{2}-(c+1 / 2)^{2}-2 m_{b} m_{c}}{\sqrt{\left[(b+1 / 2)^{2}-m_{b}^{2}\right]\left[(c+1 / 2)^{2}-m_{c}^{2}\right]}},
\end{gathered}
$$

$\cos (B), \cos (C), \cos (E)$ são deduzidos de ambas as fórmulas acima a partir de permutação ciclica de $a, b, c$ e ainda $D+E+f=2 \pi$.

Ambas as expressões acima para $3 j$ e $6 j$ símbolos são para $a, b, c, d, e, f, m_{a}, m_{b}, m_{c}$ grandes.

Para fazer a expansão assintótica desse produto, vamos denotar $l_{1}=j \lambda_{1}, l_{2}=j \lambda_{2}$, $l=j \lambda, m_{1}=j \mu_{1}, m_{2}=j \mu_{2}$ e $m=j \mu$ e adaptando as fórmulas de Ponzano Regge, teremos para $j>>0$ :

- $6 j-$ símbolo:

$\left\{\begin{array}{ccc}j \lambda_{1} & j \lambda_{2} & j \lambda \\ j & j & j\end{array}\right\} \sim \frac{1}{\sqrt{12 \pi \mathbf{V}}} \cos \left(j \lambda_{1} \theta_{12}+j \lambda_{2} \theta_{13}+j \lambda \theta_{14}+j \theta_{34}+j \theta_{24}+j \theta_{23}+\pi / 4\right)$

E para esse caso $\mathbf{V}=\frac{j^{3}}{12} \sqrt{\Delta-\lambda_{1}^{2} \lambda_{2}^{2} \lambda^{2}}$, onde $\Delta=\left(\lambda_{1}+\lambda_{2}+\lambda\right)\left(-\lambda_{1}+\lambda_{2}+\lambda\right)\left(\lambda_{1}-\lambda_{2}+\lambda\right)\left(\lambda_{1}+\lambda_{2}-\lambda\right)$

- $3 j-$ símbolo:

$$
\left(\begin{array}{ccc}
j \lambda_{1} & j \lambda_{2} & j \lambda \\
-j \mu_{1} & -j \mu_{2} & j \mu
\end{array}\right) \sim(2 \pi \mathfrak{A})^{-1 / 2}(-1)^{j \lambda_{1}+j \lambda_{2}-j \lambda+1 / 2}
$$

\footnotetext{
${ }^{2}$ pensamos que a componente $z$ está associada a direção de $P_{4}$ indo para infinito.
} 


$$
\cos \left(j \lambda_{1} \theta_{\lambda_{1}}+j \lambda_{2} \theta_{\lambda_{2}}+j \lambda_{3} \theta_{\lambda_{3}}+j \mu_{2} D-j \mu_{1} E+\pi / 4\right),
$$

onde a área nesse caso é dada por $\mathfrak{A}=\frac{j^{2}}{4} \sqrt{\widetilde{\Delta}}$, onde

$$
\begin{array}{r}
\widetilde{\Delta}=\left(\lambda_{1}^{2}-\mu_{1}^{2}\right)\left(-\lambda_{1}^{2}+\mu_{1}^{2}+\lambda_{2}^{2}-\mu_{2}^{2}+\lambda^{2}-\mu^{2}\right)+ \\
\left(\lambda_{2}^{2}-\mu_{2}^{2}\right)\left(\lambda_{1}^{2}-\mu_{1}^{2}-\lambda_{2}^{2}+\mu_{2}^{2}+\lambda^{2}-\mu^{2}\right)+ \\
\left(\lambda^{2}-\mu^{2}\right)\left(\lambda_{1}^{2}-\mu_{1}^{2}+\lambda_{2}^{2}-\mu_{2}^{2}-\lambda^{2}+\mu^{2}\right) .
\end{array}
$$

\subsubsection{Preparando o produto de Stratonovich padrão para a explo- ração assintótica}

Voltamos ao produto de Stratonovich 3.1.1 e substituimos as fórmulas fornecidas da seção anterior. Obtemos então:

$$
\begin{aligned}
& Y_{j \lambda_{1}}^{j \mu_{1}} *_{1} Y_{j \lambda_{2}}^{j \mu_{2}}= \sum_{j \lambda=j\left|\lambda_{1}-\lambda_{2}\right|}^{j \lambda_{\max }}(-1)^{2 j+j \mu} \sqrt{(2 j+1)\left(2 j \lambda_{1}+1\right)\left(2 j \lambda_{2}+1\right)(2 j \lambda+1)} . \\
& {\left[\begin{array}{ccc}
j \lambda_{1} & j \lambda_{2} & j \lambda \\
j \mu_{1} & j \mu_{2} & j \mu
\end{array}\right][j] . Y_{j \lambda}^{j \mu} } \\
&= \sum_{j \lambda=j\left|\lambda_{1}-\lambda_{2}\right|}^{j \lambda_{\max }}(-1)^{2 j+j \mu} \sqrt{(2 j+1)\left(2 j \lambda_{1}+1\right)\left(2 j \lambda_{2}+1\right)(2 j \lambda+1)} . \\
&\left\{\begin{array}{ccc}
j \lambda_{1} & j \lambda_{2} & j \lambda \\
j & j & j
\end{array}\right\} \cdot\left(\begin{array}{ccc}
j \lambda_{1} & j \lambda_{2} & j \lambda \\
-j \mu_{1} & -j \mu_{2} & j \mu
\end{array}\right) Y_{j \lambda}^{j \mu},
\end{aligned}
$$

onde $\lambda_{\max }=\min \left\{2, \lambda_{1}+\lambda_{2}\right\}$.

Como $j>>0$ podemos simplicar por:

$$
\begin{gathered}
Y_{j \lambda_{1}}^{j \mu_{1}} *_{1} Y_{j \lambda_{2}}^{j \mu_{2}} \sim \sum_{j \lambda=j\left|\lambda_{1}-\lambda_{2}\right|}^{j \lambda_{\max }}(-1)^{2 j+j \mu} \sqrt{(2 j)\left(2 j \lambda_{1}\right)\left(2 j \lambda_{2}\right)(2 j \lambda)} \cdot \frac{1}{\sqrt{12 \pi \mathbf{V}}} . \\
\cos \left(j \lambda_{1} \theta_{12}+j \lambda_{2} \theta_{13}+j \lambda \theta_{14}+j \theta_{34}+j \theta_{24}+j \theta_{23}+\pi / 4\right) . \\
(2 \pi \mathfrak{A})^{-1 / 2}(-1)^{j \lambda_{1}+j \lambda_{2}-j \lambda+1 / 2} \cos \left(j \lambda_{1} \theta_{\lambda_{1}}+j \lambda_{2} \theta_{\lambda_{2}}+j \lambda_{3} \theta_{\lambda_{3}}+j \mu_{2} D-j \mu_{1} E+\pi / 4\right) . Y_{j \lambda}^{j \mu}
\end{gathered}
$$


Temos uma fórmula, encontrada no livro do Varshalovich [21], para o harmônico esférico $Y_{l}^{m}(\vartheta, \phi)$ no caso: $l>>1$ e $l>>m \geq 0$, para $\epsilon \leq \vartheta \leq \pi-\epsilon(0<\epsilon<<1 / l)$ e $0 \leq \phi<2 \pi$ pode ser aproximado por:

$$
Y_{l}^{m}(\vartheta, \phi) \approx \frac{e^{i m \phi}}{\pi} \frac{\cos \left[(2 l+1) \frac{\vartheta}{2}+(2 m-1) \frac{\pi}{4}\right]}{\sqrt{\operatorname{sen} \vartheta}}+O\left(\frac{1}{l}\right) .
$$

O termo $O\left(\frac{1}{l}\right)=O\left(\vartheta, m_{3}\right)$ depende de $m$ e $\vartheta$, de qualquer maneira seria um termo de ordem superior em $1 / j$, quando considerado $l=j \lambda$.

Usando a expressão do livro de Varsalovich [21] para $l=j \lambda$ e $m=j \mu$ e agregando à expressão do produto:

$$
\begin{gathered}
Y_{j \lambda_{1}}^{j \mu_{1}} *_{1} Y_{j \lambda_{2}}^{j \mu_{2}} \sim \sum_{j \lambda=j\left|\lambda_{1}-\lambda_{2}\right|}^{j \lambda_{\max }}(-1)^{2 j+j \mu} \sqrt{(2 j)\left(2 j \lambda_{1}\right)\left(2 j \lambda_{2}\right)(2 j \lambda)} \cdot \frac{1}{\sqrt{12 \pi \mathbf{V}}} \cos \left(\Sigma_{1}\right) . \\
(2 \pi \mathfrak{A})^{-1 / 2}(-1)^{j \lambda_{1}+j \lambda_{2}-j \lambda+1 / 2} \cos \left(\Sigma_{2}\right) \cdot\left(\frac{e^{i j \mu \phi}}{\pi} \frac{\cos \left(\Sigma_{3}\right)}{\sqrt{\operatorname{sen\vartheta }}}+O\left(\frac{1}{j \lambda}\right)\right)
\end{gathered}
$$

onde

$$
\begin{gathered}
\Sigma_{1}=\left(j \lambda_{1} \theta_{12}+j \lambda_{2} \theta_{13}+j \lambda \theta_{14}+j \theta_{34}+j \theta_{24}+j \theta_{23}+\pi / 4\right) ; \\
\Sigma_{2}=\left(j \lambda_{1} \theta_{\lambda_{1}}+j \lambda_{2} \theta_{\lambda_{2}}+j \lambda \theta_{\lambda}+j \mu_{2} D-j \mu_{1} E+\pi / 4\right) ; \\
\Sigma_{3}=\left((2 j \lambda+1) \frac{\vartheta}{2}+(2 j \mu-1) \frac{\pi}{4}\right) .
\end{gathered}
$$

Agora substituindo $\mathbf{V}$ e $\mathfrak{A}$, temos:

$$
\begin{gathered}
Y_{j \lambda_{1}}^{j \mu_{1}} *_{1} Y_{j \lambda_{2}}^{j \mu_{2}} \sim \frac{(-1)^{j\left(2+\mu+\lambda_{1}+\lambda_{2}\right)+1 / 2} 4 \sqrt{2 \lambda_{1} \lambda_{2}}}{\pi} \\
\frac{1}{\sqrt{j}} \sum_{j \lambda=j\left|\lambda_{1}-\lambda_{2}\right|}^{j \lambda_{\max }} \frac{\sqrt{\lambda} e^{-i j \pi \lambda} \cos \left(\Sigma_{1}\right) \cos \left(\Sigma_{2}\right)}{\left[\Delta\left(\widetilde{\Delta}-\sqrt{\lambda_{1}^{2} \lambda_{2}^{2} \lambda^{2}}\right)\right]^{1 / 4}} \\
\left(\frac{e^{i j \mu \phi}}{\pi} \frac{\cos \left(\Sigma_{3}\right)}{\sqrt{\operatorname{sen\vartheta }}}+O\left(\frac{1}{j}\right)\right) .
\end{gathered}
$$

Como podemos ver esse somatório é bastante complicado para ser estudado. E como queremos explorar a expressão assintótica do produto, iremos aproximar esse somatório por uma integral: 


$$
\begin{gathered}
Y_{j \lambda_{1}}^{j \mu_{1}} *_{1} Y_{j \lambda_{2}}^{j \mu_{2}} \sim \frac{(-1)^{j\left(2+\mu+\lambda_{1}+\lambda_{2}\right)+1 / 2} 4 \sqrt{2 \lambda_{1} \lambda_{2}}}{\pi} \\
\frac{1}{\sqrt{j}} \int_{\lambda=\left|\lambda_{1}-\lambda_{2}\right|}^{\lambda_{\max }} \frac{\sqrt{\lambda} e^{-i j \pi \lambda} \cos \left(\Sigma_{1}\right) \cos \left(\Sigma_{2}\right)}{\left[\Delta\left(\widetilde{\Delta}-\sqrt{\lambda_{1}^{2} \lambda_{2}^{2} \lambda^{2}}\right)\right]^{1 / 4}} \\
\left(\frac{e^{i j \mu \phi}}{\pi} \frac{\cos \left(\Sigma_{3}\right)}{\left.\sqrt{\operatorname{sen\vartheta }}+O\left(\frac{1}{j}\right)\right) j d \lambda,}\right.
\end{gathered}
$$

onde $\lambda_{\max }=\min \left\{2, \lambda_{1}+\lambda_{2}\right\}$.

De agora em diante vamos nos concentrar nos termos de ordem mais baixa em $\frac{1}{j}$ e denotamos por:

$$
g_{1}=g_{1}(\lambda)=\frac{(-1)^{-j \lambda} \sqrt{\lambda}}{\left(\left(\Delta-\lambda_{1}^{2} \lambda_{2}^{2} \lambda^{2}\right) \widetilde{\Delta}\right)^{1 / 4}}=\frac{e^{-i j \pi \lambda} \sqrt{\lambda}}{\left[\widetilde{\Delta}\left(\Delta-\lambda_{1}^{2} \lambda_{2}^{2} \lambda^{2}\right)\right]^{1 / 4}}
$$

e

$$
g_{2}=g_{2}\left(\lambda_{1}, \lambda_{2}, \mu\right)=\frac{4(-1)^{\left.j\left(2+\mu+\lambda_{1}+\lambda_{2}\right)+1 / 2\right)} \sqrt{2 \lambda_{1} \lambda_{2}}}{\pi}
$$

Logo,

$$
\begin{gathered}
Y_{j \lambda_{1} *_{1}}^{j \mu_{1}} Y_{j \lambda_{2}}^{j \mu_{2}} \sim g_{2} \sqrt{j} \int_{\left|\lambda_{1}-\lambda_{2}\right|}^{\lambda_{\max }} g_{1} \cos \left(\Sigma_{1}\right) \cos \left(\Sigma_{2}\right)\left(\frac{e^{i j \mu \phi}}{\pi} \frac{\cos \left(\Sigma_{3}\right)}{\sqrt{\operatorname{sen\vartheta }}}+O\left(\frac{1}{j}\right)\right) d \lambda \\
=g_{2} \frac{e^{i j \mu \phi}}{\pi \sqrt{\operatorname{sen\vartheta }}} \sqrt{j} \int_{\left|\lambda_{1}-\lambda_{2}\right|}^{\lambda_{\max }} g_{1} \cos \left(\Sigma_{1}\right) \cos \left(\Sigma_{2}\right) \cos \left(\Sigma_{3}\right) d \lambda+O\left(\frac{1}{j}\right)
\end{gathered}
$$

Lembramos que $\Sigma_{1}, \Sigma_{2}$ e $\Sigma_{3}$ dependem de $\lambda$ e

$$
\cos \left(\Sigma_{s}\right)=\frac{e^{i \Sigma_{s}}+e^{-i \Sigma_{s}}}{2}, \quad s=1,2,3
$$

Faremos ainda

$$
\widetilde{g}_{2}=\frac{e^{i j \mu \phi}}{\pi \sqrt{\operatorname{sen\vartheta }}} g_{2}
$$

Com isso:

$$
Y_{j \lambda_{1}}^{j \mu_{1}} *_{1} Y_{j \lambda_{2}}^{j \mu_{2}} \sim
$$




$$
\begin{array}{r}
\sim \frac{\tilde{g}_{2}}{8} \sqrt{j} \int_{\left|\lambda_{1}-\lambda_{2}\right|}^{\lambda_{\max }} g_{1}\left(e^{i\left(\Sigma_{1}+\Sigma_{2}+\Sigma_{3}\right)}+e^{i\left(\Sigma_{1}+\Sigma_{2}-\Sigma_{3}\right)}+e^{i\left(\Sigma_{1}-\Sigma_{2}+\Sigma_{3}\right)}+\right. \\
e^{i\left(\Sigma_{1}-\Sigma_{2}-\Sigma_{3}\right)}+e^{i\left(-\Sigma_{1}+\Sigma_{2}+\Sigma_{3}\right)}+e^{i\left(-\Sigma_{1}+\Sigma_{2}-\Sigma_{3}\right)}+ \\
\left.e^{i\left(-\Sigma_{1}-\Sigma_{2}+\Sigma_{3}\right)}+e^{i\left(-\Sigma_{1}-\Sigma_{2}-\Sigma_{3}\right)}\right) d \lambda+O\left(\frac{1}{j}\right)
\end{array}
$$

Cada parcela dessa integral, denotaremos como um $P_{k}, k=1,2, \cdots, 12$. da seguinte maneira:

$$
\begin{array}{cc}
P_{1}=\int_{\left|\lambda_{1}-\lambda_{2}\right|}^{\lambda_{\max }} g_{1} e^{i\left(\Sigma_{1}+\Sigma_{2}+\Sigma_{3}\right)} d \lambda, & P_{2}=\int_{\left|\lambda_{1}-\lambda_{2}\right|}^{\lambda_{\max }} g_{1} e^{i\left(\Sigma_{1}+\Sigma_{2}-\Sigma_{3}\right)} d \lambda, \\
P_{3}=\int_{\left|\lambda_{1}-\lambda_{2}\right|}^{\lambda_{\max }} g_{1} e^{i\left(\Sigma_{1}-\Sigma_{2}+\Sigma_{3}\right)} d \lambda, & P_{4}=\int_{\left|\lambda_{1}-\lambda_{2}\right|}^{\lambda_{\max }} g_{1} e^{i\left(\Sigma_{1}-\Sigma_{2}-\Sigma_{3}\right)} d \lambda, \\
P_{5}=\int_{\left|\lambda_{1}-\lambda_{2}\right|}^{\lambda_{\max }} g_{1} e^{i\left(-\Sigma_{1}+\Sigma_{2}+\Sigma_{3}\right)} d \lambda, & P_{6}=\int_{\left|\lambda_{1}-\lambda_{2}\right|}^{\lambda_{\max }} g_{1} e^{i\left(-\Sigma_{1}+\Sigma_{2}-\Sigma_{3}\right)} d \lambda \\
P_{7}=\int_{\left|\lambda_{1}-\lambda_{2}\right|}^{\lambda_{\max }} g_{1} e^{i\left(-\Sigma_{1}-\Sigma_{2}+\Sigma_{3}\right)} d \lambda, & P_{8}=\int_{\left|\lambda_{1}-\lambda_{2}\right|}^{\lambda_{\max }} g_{1} e^{i\left(-\Sigma_{1}-\Sigma_{2}-\Sigma_{3}\right)} d \lambda,
\end{array}
$$

Observemos aqui o seguinte:

$$
P_{1}=\overline{P_{8}}, \quad P_{2}=\overline{P_{7}}, \quad P_{3}=\overline{P_{6}}, \quad P_{4}=\overline{P_{5}},
$$

Recapitulando: o que estamos explorando nesse capítulo é uma comparação entre os produtos de Stratonovich e de Berezin. O que fizemos até aqui é encontrar uma expressão assintótica para o produto de Stratonovich. O que faremos depois é encontrar uma expressão assintótica para o produto de Berezin e comparar esses dois produtos. Mas o que nos interessa é a comparação apenas na ordem mais baixa (ou "ordem zero"), pois o termo que possui $O(1 / j)$ já é mais complicado. Então em primeira ordem iremos desprezá-lo. Mais ainda, se conseguirmos mostrar a diferença desses produtos já na primeira ordem, então teremos algo relevante, sem ter que considerar os termos de ordem maior em $1 / j$.

\subsubsection{Preparando o produto de Berezin padrão para a exploração assintótica}

Vamos então, agora, fazer o mesmo procedimento feito para o produto twisted de Stratonovich, para o caso Berezin e obervar o que obtemos. 
Para o produto 3.1.2 vamos desenvolver o fator $\frac{b_{l}^{n}}{b_{l_{1}}^{n} b_{l_{2}}^{n}}$ para facilitar a análise:

$$
\begin{gathered}
\left(\frac{b_{l}^{n}}{b_{l_{1}}^{n} b_{l_{2}}^{n}}\right)^{2}=\frac{\left(\begin{array}{c}
n+l_{1}+1 \\
l_{1}
\end{array}\right)\left(\begin{array}{c}
n+l_{2}+1 \\
l_{2}
\end{array}\right)\left(\begin{array}{l}
n \\
l
\end{array}\right)}{\left(\begin{array}{l}
n \\
l_{1}
\end{array}\right)\left(\begin{array}{l}
n \\
l_{2}
\end{array}\right)\left(\begin{array}{c}
n+l+1 \\
l
\end{array}\right)} \\
=\frac{\left(n+l_{1}+1\right) !\left(n+l_{2}+1\right) ! n !}{l_{1} !(n+1) ! l_{2} !(n+1) ! l !(n-l) !} \frac{l_{1} !\left(n-l_{1}\right) ! l_{2} !\left(n-l_{2}\right) ! l !(n+1) !}{n ! n !(n+l+1) !} \\
\frac{\left(n+l_{1}+1\right) !\left(n+l_{2}+1\right) !\left(n-l_{1}\right) !\left(n-l_{2}\right) !}{(n+1) ! n !(n-l) !(n+l+1) !}
\end{gathered}
$$

Usaremos novamente o fato de que $n=2 j>>0$ e também há uma aproximação assintótica (fórmula de Stirling) para $n ! \approx \sqrt{2 \pi n}\left(\frac{n}{e}\right)^{n}$,

$$
\begin{gathered}
\left(\frac{b_{l}^{n}}{b_{l_{1}}^{n} b_{l_{2}}^{n}}\right)^{2}=\frac{\left(n+l_{1}\right) !\left(n+l_{2}\right) !\left(n-l_{1}\right) !\left(n-l_{2}\right) !}{(n !)^{2}(n-l) !(n+l) !} \\
\approx \frac{\sqrt{2 \pi\left(n+l_{1}\right)}\left(\frac{n+l_{1}}{e}\right)^{\left(n+l_{1}\right)} \sqrt{2 \pi\left(n+l_{2}\right)}\left(\frac{n+l_{2}}{e}\right)^{\left(n+l_{2}\right)} \sqrt{2 \pi\left(n-l_{1}\right)}\left(\frac{n-l_{1}}{e}\right)^{\left(n-l_{1}\right)} \sqrt{2 \pi\left(n-l_{2}\right)}\left(\frac{n-l_{2}}{e}\right)^{\left(n-l_{2}\right)}}{\left(\sqrt{2 \pi n}\left(\frac{n}{e}\right)^{n}\right)^{2} \sqrt{2 \pi(n-l)}\left(\frac{n-l}{e}\right)^{(n-l)} \sqrt{2 \pi(n+l)}\left(\frac{n+l}{e}\right)^{(n+l)}} \\
\approx \frac{4 \pi^{2} \sqrt{\left(n+l_{1}\right)\left(n-l_{1}\right)\left(n+l_{2}\right)\left(n-l_{2}\right)}\left(\frac{n+l_{1}}{e}\right)^{\left(n+l_{1}\right)}\left(\frac{n-l_{1}}{e}\right)^{\left(n-l_{1}\right)}\left(\frac{n+l_{2}}{e}\right)^{\left(n+l_{2}\right)}\left(\frac{n-l_{2}}{e}\right)^{\left(n-l_{2}\right)}}{{ }^{2 n} \sqrt{(n+l)(n-l)}\left(\frac{n+l}{e}\right)^{(n+l)}\left(\frac{n-l}{e}\right)^{(n-l)}} \\
\approx \sqrt{\frac{\left(n^{2}-l_{1}^{2}\right)\left(n^{2}-l_{2}^{2}\right)}{\left(n^{2}-l^{2}\right)}} \frac{\left(n+l_{1}\right)^{\left(n+l_{1}\right)}\left(n-l_{1}\right)^{\left(n-l_{1}\right)}\left(n+l_{2}\right)^{\left(n+l_{2}\right)}\left(n-l_{2}\right)^{\left(n-l_{2}\right)}}{n^{(2 n+1)}(n+l)^{(n+l)}(n-l)^{(n-l)}}
\end{gathered}
$$

Enfim, usaremos as condições: $l_{k}=j \lambda_{k}$ para $k=1,2, l=j \lambda$ e $n=2 j$,

$$
\begin{gathered}
\left(\frac{b_{j \lambda}^{2 j}}{b_{j \lambda_{1}}^{2 j} b_{j \lambda_{2}}^{2 j}}\right)=\frac{1}{2^{2 j} \sqrt{2}}\left(\frac{\left(4-\lambda_{1}^{2}\right)\left(4-\lambda_{2}^{2}\right)}{\left(4-\lambda^{2}\right)}\right)^{1 / 4} \\
{\left[\frac{\left(2+\lambda_{1}\right)^{\left(2+\lambda_{1}\right)}\left(2-\lambda_{1}\right)^{\left(2-\lambda_{1}\right)}\left(2+\lambda_{2}\right)^{\left(2+\lambda_{2}\right)}\left(2-\lambda_{2}\right)^{\left(2-\lambda_{2}\right)}}{(2+\lambda)^{(2+\lambda)}(2-\lambda)^{(2+\lambda)}}\right]^{j / 2}}
\end{gathered}
$$

Agora que temos uma fórmula aproximada para os $\left(\frac{b_{j \lambda}^{2 j}}{b_{j \lambda_{1}}^{2 j} b_{j \lambda_{2}}^{2 j}}\right)$, podemos tornar ao produto de Berezin 3.1.2 e fazer mais essa subtituição, além de todas as feitas para 
o produto de Stratonovich, que serão análogas para esse produto também. Obtemos então:

$$
\begin{gathered}
Y_{j \lambda_{1}}^{j \mu_{1}} * \vec{b} Y_{j \lambda_{2}}^{j \mu_{2}} \sim \frac{(-1)^{j\left(2+\mu+\lambda_{1}+\lambda_{2}\right)+1 / 2} 4 \sqrt{2 \lambda_{1} \lambda_{2}}}{\pi} \\
\frac{1}{\sqrt{j}} \sum_{j \lambda=j\left|\lambda_{1}-\lambda_{2}\right|}^{j \lambda_{\max }} \frac{\sqrt{\lambda} e^{-i j \pi \lambda} \cos \left(\Sigma_{1}\right) \cos \left(\Sigma_{2}\right)}{\left[\Delta\left(\widetilde{\Delta}-\sqrt{\lambda_{1}^{2} \lambda_{2}^{2} \lambda^{2}}\right)\right]^{1 / 4}} \\
\frac{1}{2^{2 j} \sqrt{2}}\left(\frac{\left(4-\lambda_{1}^{2}\right)\left(4-\lambda_{2}^{2}\right)}{\left(4-\lambda^{2}\right)}\right)^{1 / 4} \\
{\left[\frac{\left(2+\lambda_{1}\right)^{\left(2+\lambda_{1}\right)}\left(2-\lambda_{1}\right)^{\left(2-\lambda_{1}\right)}\left(2+\lambda_{2}\right)^{\left(2+\lambda_{2}\right)}\left(2-\lambda_{2}\right)^{\left(2-\lambda_{2}\right)}}{(2+\lambda)^{(2+\lambda)}(2-\lambda)^{(2+\lambda)}}\right]^{j / 2}} \\
\left(\frac{e^{i j \mu \phi}}{\pi} \frac{\cos \left(\Sigma_{3}\right)}{\sqrt{\operatorname{sen\vartheta }}}+O\left(\frac{1}{j}\right)\right) .
\end{gathered}
$$

Vamos denotar por $g_{3}=g_{3}\left(\lambda_{1}, \lambda_{2}\right)$ a seguinte parcela:

$$
\begin{array}{r}
g_{3}=\left[\left(2+\lambda_{1}\right)^{\left(2+\lambda_{1}\right)}\left(2-\lambda_{1}\right)^{\left(2-\lambda_{1}\right)}\left(2+\lambda_{2}\right)^{\left(2+\lambda_{2}\right)}\left(2-\lambda_{2}\right)^{\left(2-\lambda_{2}\right)}\right]^{j / 2} . \\
\frac{\left[\left(4-\lambda_{1}^{2}\right)\left(4-\lambda_{2}^{2}\right)\right]^{1 / 4}}{2^{2 j} \sqrt{2}}
\end{array}
$$

Simplificando esse somatório usando as funções $g_{1}, g_{2}$ e $g_{3}$, dadas por 3.1.3, 3.1.4 e 3.1.6, respectivamente, obtemos:

$Y_{j \lambda_{1}}^{j \mu_{1}} * \vec{b} Y_{j \lambda_{2}}^{j \mu_{2}} \approx \frac{1}{\sqrt{j}} \sum_{j \lambda=j\left|\lambda_{1}-\lambda_{2}\right|}^{j \lambda_{\max }} \frac{g_{1} g_{2} g_{3} \cos \left(\Sigma_{1}\right) \cos \left(\Sigma_{2}\right)}{\left(4-\lambda^{2}\right)^{1 / 4}\left[(2+\lambda)^{(2+\lambda)}(2-\lambda)^{(2-\lambda)}\right]^{j / 2}}\left(\frac{e^{i j \mu \phi}}{\pi} \frac{\cos \left(\Sigma_{3}\right)}{\sqrt{\operatorname{sen} \vartheta}}+O(1 / j)\right)$.

Novamente, aproximaremos esse somatório pela integral, considerando apenas os termos de ordem mais baixa em $1 / j$, e usaremos a mesma idéia de 3.1.8, teremos:

$$
\begin{gathered}
Y_{j \lambda_{1}}^{j \mu_{1}} * \vec{b} Y_{j \lambda_{2}}^{j \mu_{2}} \sim \frac{\widetilde{g}_{2} g_{3}}{8} \sqrt{j} \int_{\left|\lambda_{1}-\lambda_{2}\right|}^{\lambda_{\max }} \frac{g_{1}}{\left(4-\lambda^{2}\right)^{1 / 4}\left[(2+\lambda)^{(2+\lambda)}(2-\lambda)^{(2-\lambda)]^{j / 2}}\right.} \\
\left(e^{i\left(\Sigma_{1}+\Sigma_{2}+\Sigma_{3}\right)}+e^{i\left(\Sigma_{1}+\Sigma_{2}-\Sigma_{3}\right)}+e^{i\left(\Sigma_{1}-\Sigma_{2}+\Sigma_{3}\right)}+e^{i\left(\Sigma_{1}-\Sigma_{2}-\Sigma_{3}\right)}+\right. \\
\left.e^{i\left(-\Sigma_{1}+\Sigma_{2}+\Sigma_{3}\right)}+e^{i\left(-\Sigma_{1}+\Sigma_{2}-\Sigma_{3}\right)}+e^{i\left(-\Sigma_{1}-\Sigma_{2}+\Sigma_{3}\right)}+e^{i\left(-\Sigma_{1}-\Sigma_{2}-\Sigma_{3}\right)}\right) d \lambda
\end{gathered}
$$


Denotaremos cada parcela dessa integral por $Q_{k}$ para $k=1,2, \cdots, 8$, da seguinte maneira:

$$
\begin{aligned}
& Q_{1}=\int_{\left|\lambda_{1}-\lambda_{2}\right|}^{\lambda_{\max }} \frac{g_{1} e^{i\left(\Sigma_{1}+\Sigma_{2}+\Sigma_{3}\right)}}{\left(4-\lambda^{2}\right)^{1 / 4}\left[(2+\lambda)^{(2+\lambda)}(2-\lambda)^{(2-\lambda)}\right]^{j / 2}} d \lambda \\
& Q_{2}=\int_{\left|\lambda_{1}-\lambda_{2}\right|}^{\lambda_{\max }} \frac{g_{1} e^{i\left(\Sigma_{1}+\Sigma_{2}-\Sigma_{3}\right)}}{\left(4-\lambda^{2}\right)^{1 / 4}\left[(2+\lambda)^{(2+\lambda)}(2-\lambda)^{(2-\lambda)}\right] j / 2} d \lambda \\
& Q_{3}=\int_{\left|\lambda_{1}-\lambda_{2}\right|}^{\lambda_{\max }} \frac{g_{1} e^{i\left(\Sigma_{1}-\Sigma_{2}+\Sigma_{3}\right)}}{\left(4-\lambda^{2}\right)^{1 / 4}\left[(2+\lambda)^{(2+\lambda)}(2-\lambda)^{(2-\lambda)}\right]^{j / 2}} d \lambda ; \\
& Q_{4}=\int_{\left|\lambda_{1}-\lambda_{2}\right|}^{\lambda_{\max }} \frac{g_{1} e^{i\left(\Sigma_{1}-\Sigma_{2}-\Sigma_{3}\right)}}{\left(4-\lambda^{2}\right)^{1 / 4}\left[(2+\lambda)^{(2+\lambda)}(2-\lambda)^{(2-\lambda)}\right]^{j / 2}} d \lambda \\
& Q_{5}=\int_{\left|\lambda_{1}-\lambda_{2}\right|}^{\lambda_{\max }} \frac{g_{1} e^{i\left(-\Sigma_{1}+\Sigma_{2}+\Sigma_{3}\right)}}{\left(4-\lambda^{2}\right)^{1 / 4}\left[(2+\lambda)^{(2+\lambda)}(2-\lambda)^{(2-\lambda)}\right]^{j / 2}} d \lambda \\
& Q_{6}=\int_{\left|\lambda_{1}-\lambda_{2}\right|}^{\lambda_{\max }} \frac{g_{1} e^{i\left(-\Sigma_{1}+\Sigma_{2}-\Sigma_{3}\right)}}{\left(4-\lambda^{2}\right)^{1 / 4}\left[(2+\lambda)^{(2+\lambda)}(2-\lambda)^{(2-\lambda)}\right]^{j / 2}} d \lambda \\
& Q_{7}=\int_{\left|\lambda_{1}-\lambda_{2}\right|}^{\lambda_{\max }} \frac{g_{1} e^{i\left(-\Sigma_{1}-\Sigma_{2}+\Sigma_{3}\right)}}{\left(4-\lambda^{2}\right)^{1 / 4}\left[(2+\lambda)^{(2+\lambda)}(2-\lambda)^{(2-\lambda)}\right]^{j / 2}} d \lambda \\
& Q_{8}=\int_{\left|\lambda_{1}-\lambda_{2}\right|}^{\lambda_{\max }} \frac{g_{1} e^{i\left(-\Sigma_{1}-\Sigma_{2}-\Sigma_{3}\right)}}{\left(4-\lambda^{2}\right)^{1 / 4}\left[(2+\lambda)^{(2+\lambda)}(2-\lambda)^{(2-\lambda)}\right]^{j / 2}} d \lambda .
\end{aligned}
$$

Observemos aqui o seguinte:

$$
Q_{1}=\overline{Q_{8}}, \quad Q_{2}=\overline{Q_{7}}, \quad Q_{3}=\overline{Q_{6}}, \quad Q_{4}=\overline{Q_{5}} .
$$

Conclusão: se olharmos para a expressão assintótica de Stratonovich:

$$
\begin{gathered}
Y_{j \lambda_{1}}^{j \mu_{1}} *_{1} Y_{j \lambda_{2}}^{j \mu_{2}} \sim \frac{1}{\sqrt{j}} \sum_{j \lambda=j\left|\lambda_{1}-\lambda_{2}\right|}^{j \lambda_{\max }} g_{2} g_{1} \cos \left(\Sigma_{1}\right) \cos \left(\Sigma_{2}\right)\left(\frac{e^{-i j \mu \phi}}{\pi} \frac{\cos \left(\Sigma_{3}\right)}{\sqrt{\operatorname{sen} \vartheta}}\right) \\
\sim \frac{\widetilde{g}_{2}}{8} \sqrt{j} \int_{\left|\lambda_{1}-\lambda_{2}\right|}^{\lambda_{\max }} g_{1}\left(e^{i\left(\Sigma_{1}+\Sigma_{2}+\Sigma_{3}\right)}+e^{i\left(\Sigma_{1}+\Sigma_{2}-\Sigma_{3}\right)}+e^{i\left(\Sigma_{1}-\Sigma_{2}+\Sigma_{3}\right)}+e^{i\left(\Sigma_{1}-\Sigma_{2}-\Sigma_{3}\right)}+\right. \\
\left.e^{i\left(-\Sigma_{1}+\Sigma_{2}+\Sigma_{3}\right)}+e^{i\left(-\Sigma_{1}+\Sigma_{2}-\Sigma_{3}\right)}+e^{i\left(-\Sigma_{1}-\Sigma_{2}+\Sigma_{3}\right)}+e^{i\left(-\Sigma_{1}-\Sigma_{2}-\Sigma_{3}\right)}\right) d \lambda(3.1 .8)
\end{gathered}
$$


E para a expressão assintótica de Berezin:

$$
\begin{array}{r}
Y_{j \lambda_{1}}^{j \mu_{1}} * \vec{b} Y_{j \lambda_{2}}^{j \mu_{2}} \sim \frac{1}{\sqrt{j}} \sum_{j \lambda=j\left|\lambda_{1}-\lambda_{2}\right|}^{j \lambda_{\max }} \frac{g_{1} g_{2} g_{3} \cos \left(\Sigma_{1}\right) \cos \left(\Sigma_{2}\right)}{\left(\frac{e^{-i j \mu \phi}}{\pi} \frac{\cos \left(\Sigma_{3}\right)}{\sqrt{\operatorname{sen} \vartheta}}\right)} \\
\sim \frac{\widetilde{g}_{2} g_{3}}{8} \sqrt{j} \int_{\left|\lambda_{1}-\lambda_{2}\right|}^{\lambda_{\max }} \frac{g_{1}\left[(2+\lambda)^{(2+\lambda)}(2-\lambda)^{(2-\lambda)] j / 2}\right.}{\left(4-\lambda^{2}\right)^{1 / 4}\left[(2+\lambda)^{(2+\lambda)}(2-\lambda)^{(2-\lambda)}\right] j / 2} \\
\left(e^{i\left(\Sigma_{1}+\Sigma_{2}+\Sigma_{3}\right)}+e^{i\left(\Sigma_{1}+\Sigma_{2}-\Sigma_{3}\right)}+e^{i\left(\Sigma_{1}-\Sigma_{2}+\Sigma_{3}\right)}+e^{i\left(\Sigma_{1}-\Sigma_{2}-\Sigma_{3}\right)}+\right. \\
\left.e^{i\left(-\Sigma_{1}+\Sigma_{2}+\Sigma_{3}\right)}+e^{i\left(-\Sigma_{1}+\Sigma_{2}-\Sigma_{3}\right)}+e^{i\left(-\Sigma_{1}-\Sigma_{2}+\Sigma_{3}\right)}+e^{i\left(-\Sigma_{1}-\Sigma_{2}-\Sigma_{3}\right)}\right) d \lambda .
\end{array}
$$

Essas expressões 3.1.7 e 3.1.9 parecem claramente distintas no limite assintótico, $j \rightarrow \infty$ na ordem mais baixa em $1 / j$. Para termos certeza dessa distinção, vamos calcular um exemplo particular no final desse capítulo. Porém, antes, vamos fazer a mesma exploração do produto pontual na próxima secção para compararmos os três produtos finais.

\subsubsection{Expressão assintótica do produto pontual}

Embora o produto clássico de funções na esfera, o produto pontual e o colchetes de Poisson, são definidos para funcções gerais na esfera, é útil obter fórmulas para esses produtos clássicos decompostos na base ortonormal de esféricos harmôni$\left.\cos <Y_{l}^{m}, Y_{l^{\prime}}^{m^{\prime}}\right\rangle=\delta_{l l^{\prime}} \delta_{m m^{\prime}}$. Seguindo [12], apresentaremos como definição do produto pontual, o que vem a ser uma proposição em [12], para objetivo de sua exploração assintótica na tese.

Definição 3.1.2. O produto pontual de harmônicos esféricos se decompoem na base de harmônicos esféricos de acordo com a seguinte fórmula:

$$
Y_{l_{1}}^{m_{1}} \cdot Y_{l_{2}}^{m_{2}}=\sum_{l=\left|l_{1}-l_{2}\right|, l \equiv l_{1}+l 2(\bmod 2)}^{l_{1}+l_{2}} \sqrt{\frac{\left(2 l_{1}+1\right)\left(2 l_{2}+1\right)}{(2 l+1)}} C_{m_{1}, m_{2}, m}^{l_{1}, l_{2}, l} C_{0,0,0}^{l_{1}, l_{2}, l} Y_{l}^{m},
$$


onde o coeficiente de Clebsh-Gordan $C_{m_{1}, m_{2}, m}^{l_{1}, l_{2}, l}$ é dado por

$$
C_{m_{1}, m_{2}, m}^{l_{1}, l_{2}, l}=(-1)^{l_{1}-l_{2}+m} \sqrt{2 l+1}\left(\begin{array}{ccc}
l_{1} & l_{2} & l \\
m_{1} & m_{2} & -m
\end{array}\right) .
$$

A partir dessa definição vamos abrir esse somatório para encontrar uma expressão assintótica, da mesma maneira como foi feito para os trikernels de Stratonovich e de Berezin.

$$
\begin{aligned}
& Y_{l_{1}}^{m_{1}} . Y_{l_{2}}^{m_{2}}=\sum_{l=\left|l_{1}-l_{2}\right|, l \equiv l_{1}+l 2(\bmod 2)}^{l_{1}+l_{2}} \sqrt{\frac{\left(2 l_{1}+1\right)\left(2 l_{2}+1\right)}{(2 l+1)}}(-1)^{l_{1}-l_{2}+m} \sqrt{2 l+1}\left(\begin{array}{ccc}
l_{1} & l_{2} & l \\
m_{1} & m_{2} & -m
\end{array}\right) \\
& (-1)^{l_{1}-l_{2}} \sqrt{2 l+1}\left(\begin{array}{ccc}
l_{1} & l_{2} & l \\
0 & 0 & 0
\end{array}\right) \cdot Y_{l}^{m} \\
& =\sum \sqrt{\left(2 l_{1}+1\right)\left(2 l_{2}+1\right)(2 l+1)}(-1)^{2 l_{1}+2 l_{2}+m}\left(\begin{array}{ccc}
l_{1} & l_{2} & l \\
m_{1} & m_{2} & -m
\end{array}\right)\left(\begin{array}{ccc}
l_{1} & l_{2} & l \\
0 & 0 & 0
\end{array}\right) Y_{l}^{m}
\end{aligned}
$$

Nota-se que o produto pontual é independente de $j$, porém ao fazermos a assintótica $j \rightarrow \infty$, será "importado"para esse produto ao fazermos $l_{i}=j \lambda_{i}, m_{i}=j \mu_{i}$ para $i=1,2$ ,$l=j \lambda$ e $m=j \mu$. Assim continuando temos:

$$
\begin{aligned}
Y_{l_{1}}^{m_{1}} . Y_{l_{2}}^{m_{2}}= & \sum_{j \lambda=j\left|\lambda_{1}-\lambda_{2}\right|, j \lambda \equiv j \lambda_{1}+j \lambda_{2}(\bmod 2)}^{j\left(\lambda_{1}+\lambda_{2}\right)} \sqrt{\left(2 j \lambda_{1}+1\right)\left(2 j \lambda_{2}+1\right)(2 j \lambda+1)}(-1)^{2 j \lambda_{1}+2 j \lambda_{2}+j \mu} \\
& \left(\begin{array}{ccc}
j \lambda_{1} & j \lambda_{2} & j \lambda \\
j \mu_{1} & j \mu_{2} & -j \mu
\end{array}\right)\left(\begin{array}{ccc}
j \lambda_{1} & j \lambda_{2} & j \lambda \\
0 & 0 & 0
\end{array}\right) Y_{j \lambda}^{j \mu}
\end{aligned}
$$

Usando, mais uma vez, $j>>1$, as fórmulas assintóticas de Ponzano-Regge [17] e a aproximação pela integral, obtemos:

$$
\begin{aligned}
Y_{l_{1}}^{m_{1}} . Y_{l_{2}}^{m_{2}} \simeq & j^{3 / 2} \frac{\sqrt{2 \lambda_{1} \lambda_{2}}}{4 \pi} \int_{\left|\lambda_{1}-\lambda_{2}\right|}^{\lambda_{\max }} \frac{\sqrt{\lambda}}{\mathfrak{A}}(-1)^{1+j\left(7 \lambda_{1}-\lambda_{2}-\lambda+2 \mu\right) / 2}\left[1+(-1)^{j\left(\lambda_{1}+\lambda_{2}+\lambda\right)}\right] \\
& \left(e^{i \Sigma_{2}}+e^{-i \Sigma_{2}}\right)\left(\frac{e^{i j \mu \phi}}{2 \pi \sqrt{\operatorname{sen}(\vartheta)}}\left(e^{i \Sigma_{3}}+e^{-i \Sigma_{3}}\right)+O(1 / j)\right) j d \lambda .
\end{aligned}
$$

Relembrando que

$$
\Sigma_{3}=(2 j \lambda+1) \frac{\vartheta}{2}+(2 j \mu-1) \frac{\pi}{4}
$$


e

$$
\Sigma_{2}=j\left(\lambda_{1} \theta_{\lambda_{1}}+\lambda_{2} \theta_{\lambda_{2}}+\lambda \theta_{\lambda}+\mu_{2} D-\mu_{1} E\right)+\pi / 4
$$

Continuando a explorar a menor ordem em $1 / j$ :

$$
\begin{gathered}
Y_{j \lambda_{1}}^{j \mu_{1}} \cdot Y_{j \lambda_{2}}^{j \mu_{2}} \approx \frac{e^{i j \mu \phi} \sqrt{2 \lambda_{1} \lambda_{2}}}{2 \pi^{2} \sqrt{\operatorname{sen}(\vartheta)}}(-1)^{1+j\left(7 \lambda_{1}-\lambda_{2}+2 \mu\right) / 2} \\
j^{5 / 2} \int_{\left|\lambda_{1}-\lambda_{2}\right|}^{\lambda_{\max }}(-1)^{-j \lambda / 2} \sqrt{\frac{\lambda}{\widetilde{\Delta}}}\left[1+(-1)^{j\left(\lambda_{1}+\lambda_{2}+\lambda\right)}\right] \\
\left(e^{i\left(\Sigma_{2}+\Sigma_{3}\right)}+e^{i\left(\Sigma_{2}-\Sigma_{3}\right)}+e^{i\left(\Sigma_{3}-\Sigma_{2}\right)}+e^{-i\left(\Sigma_{2}+\Sigma_{3}\right)}\right) d \lambda,
\end{gathered}
$$

para simplificar e facilitar a notação vamos denotar:

$$
h_{1}=h_{1}\left(\lambda_{1}, \lambda_{2}, \mu\right)=\frac{e^{i j \mu \phi} \sqrt{2 \lambda_{1} \lambda_{2}}}{2 \pi^{2} \sqrt{\operatorname{sen}(\vartheta)}}(-1)^{1+j\left(7 \lambda_{1}-\lambda_{2}+2 \mu\right) / 2}
$$

e

$$
\widetilde{h_{1}}=\widetilde{h_{1}}\left(\lambda_{1}, \lambda_{2}, \mu\right)=\frac{e^{i j \mu \phi} \sqrt{2 \lambda_{1} \lambda_{2}}}{2 \pi^{2} \sqrt{\operatorname{sen}(\vartheta)}}(-1)^{1+j\left(8 \lambda_{1}+2 \mu\right) / 2} .
$$

Com isso,

$$
\begin{gathered}
Y_{j \lambda_{1}}^{j \mu_{1}} . Y_{j \lambda_{2}}^{j \mu_{2}} \approx h_{1} j^{5 / 2} \int_{\left|\lambda_{1}-\lambda_{2}\right|}^{\lambda_{\max }} g_{1}\left(e^{i\left(\Sigma_{2}+\Sigma_{3}\right)}+e^{i\left(\Sigma_{2}-\Sigma_{3}\right)}+e^{i\left(\Sigma_{3}-\Sigma_{2}\right)}+e^{-i\left(\Sigma_{2}+\Sigma_{3}\right)}\right) d \lambda \\
+{\widetilde{h_{1}}}^{5 / 2} \int_{\left|\lambda_{1}-\lambda_{2}\right|}^{\lambda_{\max }} \widetilde{g}_{1}\left(e^{i\left(\Sigma_{2}+\Sigma_{3}\right)}+e^{i\left(\Sigma_{2}-\Sigma_{3}\right)}+e^{i\left(\Sigma_{3}-\Sigma_{2}\right)}+e^{-i\left(\Sigma_{2}+\Sigma_{3}\right)}\right) d \lambda,
\end{gathered}
$$

onde,

$$
g_{1}(\lambda)=(-1)^{-j \lambda / 2} \sqrt{\frac{\lambda}{\widetilde{\Delta}}}
$$

$\mathrm{e}$

$$
\widetilde{g}_{1}(\lambda)=(-1)^{j \lambda / 2} \sqrt{\frac{\lambda}{\widetilde{\Delta}}}
$$

Chamamos cada parcela dessas integrais por $R_{j}$ e $\widetilde{R_{j}}$, para $j=1, \cdots, 4$, da seguinte forma:

$$
\begin{aligned}
& R_{1}=\int_{\left|\lambda_{1}-\lambda_{2}\right|}^{\lambda_{\max }}(-1)^{-j \lambda / 2} \sqrt{\frac{\lambda}{\widetilde{\Delta}}} e^{i\left(\Sigma_{2}+\Sigma_{3}\right)} d \lambda, \\
& R_{2}=\int_{\left|\lambda_{1}-\lambda_{2}\right|}^{\lambda_{\max }}(-1)^{-j \lambda / 2} \sqrt{\frac{\lambda}{\widetilde{\Delta}}} e^{i\left(\Sigma_{2}-\Sigma_{3}\right)} d \lambda,
\end{aligned}
$$




$$
\begin{aligned}
& R_{3}=\int_{\left|\lambda_{1}-\lambda_{2}\right|}^{\lambda_{\max }}(-1)^{-j \lambda / 2} \sqrt{\frac{\lambda}{\widetilde{\Delta}}} e^{i\left(\Sigma_{3}-\Sigma_{2}\right)} d \lambda, \\
& R_{4}=\int_{\left|\lambda_{1}-\lambda_{2}\right|}^{\lambda_{\max }}(-1)^{-j \lambda / 2} \sqrt{\frac{\lambda}{\widetilde{\Delta}}} e^{-i\left(\Sigma_{2}+\Sigma_{3}\right)} d \lambda, \\
& \widetilde{R_{1}}=\int_{\left|\lambda_{1}-\lambda_{2}\right|}^{\lambda_{\max }}(-1)^{j \lambda / 2} \sqrt{\frac{\lambda}{\widetilde{\Delta}}} e^{i\left(\Sigma_{2}+\Sigma_{3}\right)} d \lambda, \\
& \widetilde{R_{2}}=\int_{\left|\lambda_{1}-\lambda_{2}\right|}^{\lambda_{\max }}(-1)^{j \lambda / 2} \sqrt{\frac{\lambda}{\widetilde{\Delta}}} e^{i\left(\Sigma_{2}-\Sigma_{3}\right)} d \lambda, \\
& \widetilde{R_{3}}=\int_{\left|\lambda_{1}-\lambda_{2}\right|}^{\lambda_{\max }}(-1)^{j \lambda / 2} \sqrt{\frac{\lambda}{\widetilde{\Delta}}} e^{i\left(\Sigma_{3}-\Sigma_{2}\right)} d \lambda, \\
& \widetilde{R_{4}}=\int_{\left|\lambda_{1}-\lambda_{2}\right|}^{\lambda_{\max }}(-1)^{j \lambda / 2} \sqrt{\frac{\lambda}{\widetilde{\Delta}}} e^{-i\left(\Sigma_{2}+\Sigma_{3}\right)} d \lambda .
\end{aligned}
$$

Observem ainda,

$$
R_{1}=\overline{R_{4}}, \quad R_{2}=\overline{R_{3}}, \quad \widetilde{R_{1}}=\overline{\widetilde{R_{4}}}, \quad \widetilde{R_{2}}=\overline{\widetilde{R_{4}}}
$$

\subsubsection{Exemplo para $\lambda_{1}$ e $\lambda_{2}$ particulares}

Exemplo 3.1.3. Tomemos $\lambda_{1}=1 / 2$ e $\lambda_{2}=1 / 4$. Assim $1 / 4 \leq \lambda \leq 3 / 4$. Tomemos também $\vartheta=\pi / 2, m_{1}=0=m_{2}$. Obtemos com essas escolhas as seguintes integrais.

- Para Stratonovich

$$
\begin{aligned}
Y_{j \lambda_{1}}^{j \mu_{1}} *_{1} Y_{j \lambda_{2}}^{j \mu_{2}} & \sim g_{2} \sqrt{j} \int_{1 / 4}^{3 / 4} g_{1} \cos \left(\Sigma_{1}\right) \cos \left(\Sigma_{2}\right)\left(\frac{e^{-i j \mu \phi}}{\pi} \cos \left(\Sigma_{3}\right)+O\left(\frac{1}{j \lambda}\right) d \lambda\right) \\
& \sim \widetilde{g_{2}} \sqrt{j} \int_{1 / 4}^{3 / 4} g_{1} \cos \left(\Sigma_{1}\right) \cos \left(\Sigma_{2}\right) \cos \left(\Sigma_{3}\right) d \lambda+O(1 / j)
\end{aligned}
$$

- Para Berezin

$$
\begin{aligned}
Y_{j \lambda_{1}}^{j \mu_{1}} * \vec{b} Y_{j \lambda_{2}}^{j \mu_{2}} & \sim g_{2} g_{3} \sqrt{j} \int_{1 / 4}^{3 / 4} \frac{g_{1} \cos \left(\Sigma_{1}\right) \cos \left(\Sigma_{2}\right)}{\left(4-\lambda^{2}\right)^{1 / 4}\left[(2+\lambda)^{(2+\lambda)}(2-\lambda)^{(2-\lambda)}\right] j / 2} \cdot\left(\frac{e^{-i j \mu \phi}}{\pi} \cos \left(\Sigma_{3}\right)+O(1 / j)\right) d \lambda \\
& \sim \widetilde{g}_{2} g_{3} \sqrt{j} \int_{1 / 4}^{3 / 4} \frac{g_{1} \cos \left(\Sigma_{1}\right) \cos \left(\Sigma_{2}\right) \cos \left(\Sigma_{3}\right)}{\left(4-\lambda^{2}\right)^{1 / 4}\left[(2+\lambda)^{(2+\lambda)}(2-\lambda)^{(2-\lambda)}\right] j / 2} d \lambda O(1 / j) .
\end{aligned}
$$


- Para o produto pontual

$$
Y_{j \lambda_{1}}^{j \mu_{1}} \cdot Y_{j \lambda_{2}}^{j \mu_{2}} \approx h_{1} j^{5 / 2} \int_{\left|\lambda_{1}-\lambda_{2}\right|}^{\lambda_{\max }} g_{1}\left(e^{i\left(\Sigma_{2}+\Sigma_{3}\right)}+e^{i\left(\Sigma_{2}-\Sigma_{3}\right)}+e^{i\left(\Sigma_{3}-\Sigma_{2}\right)}+e^{-i\left(\Sigma_{2}+\Sigma_{3}\right)}\right) d \lambda
$$

Como queremos estudar o limite desses produto quando $j \rightarrow \infty$, a parcela das integrais que possuem o termo $O(1 / j)$ poderá ser desprezado, já que a ordem ficará pequena.

Como podem acompanhar os cálculos detalhados no apêndice C, 3.3 , 3.4 e 3.5, todos os três produtos irão para zero quando $j \rightarrow \infty$

$$
\begin{gathered}
Y_{j \lambda_{1}}^{j \mu_{1}} *_{1} Y_{j \lambda_{2}}^{j \mu_{2}} \approx 0 \\
Y_{j \lambda_{1}}^{j \mu_{1}} * \rightarrow{ }_{b} Y_{j \lambda_{2}}^{j \mu_{2}} \approx 0 \\
Y_{j \lambda_{1}}^{j \mu_{1}} Y_{j \lambda_{2}}^{j \mu_{2}}=0
\end{gathered}
$$

A questão a ser analisada aqui é a velocidade com que cada produto tende a zero quando $j$ tende ao infinito.

E assim podemos concluir que o produto pontual será zero, já que não haverá as contribuições pela função fase. E entre Stratonovich e Berezin, chegamos em, respectivamente:

$$
\left|Y_{j \lambda_{1}}^{j \mu_{1}} *_{1} Y_{j \lambda_{2}}^{j \mu_{2}}\right| \leq N_{0} e^{-0.83088 j}
$$

e

$$
\left|Y_{j \lambda_{1}}^{j \mu_{1}} \star \rightarrow{ }_{b} Y_{j \lambda_{2}}^{j \mu_{2}}\right| \leq M_{0} e^{-1.13 j}
$$

o que nos permite conjecturar que o produto de Berezin tende a zero mais rapidamente do que o produto de Stratonovich, mesmo que essa diferença seja pequena nestas contas deste exemplo particular.

\subsection{Conclusão}

Relembramos primeiramente que o que nos levou a fazer essa exploração foi a partir do estudo feito em [12], onde a exploração assintótica foi feita para os casos em que $l$ era finito e $n \rightarrow \infty$. Em [12] notaram que a parcela de ordem zero da expansão do produto de Stratonovich-Weyl padrão coincidia com o produto pontual e a parcela de ordem primeira dessa mesma expansão coincidia com o colchete de Poisson. Porém, 
ao fazer a assintótica para o produto de Berezin padrão a parcela de ordem zero, na expansão em $1 / n$, coincidia também com o produto pontual, mas a parcela de ordem primeira, dessa expansão, já não coincidia com o colchete de Poisson. Outro fato, mais adiante em [12] que merece destaque é que os números característicos de Berezin, $b_{l}^{l}$, vão para zero quando $l$ vai pra infinito e já os número característicos de Stratonovich padrão, $c_{l} \equiv c_{l}^{n}$, são todos iguais a 1 . Essa análise foi que nos fez suspeitar que ao estudarmos a expansão assintótica dos produtos de Stratonovich e de Berezin quando $l$ e $j$ tendem a infinito, seus comportamentos não sejam coincidentes.

Em particular, o importante é destacar que com o exemplo da seção acima conseguimos elaborar uma conjectura sobre o comportamento assintótico do produto de Stratonovich e do produto de Berezin (em comparação com o produto pontual) de harmônicos esféricos quando os l's tendem a infinito linearmente com $j$. Nossa conjectura é que nesta assintótica, ambos os produtos irão a zero em "ordem zero", coincidindo com o produto pontual neste limite assintótico, sendo que o produto de Berezin tende a zero mais rapidamente que o produto de Stratonovich. Porém, informações sobre termos de ordem mais alta deste tipo de expansão assintótica, ou informações mais precisas e detalhadas do que acontece com esses produtos de maneira mais genérica, quando $j \rightarrow \infty$, serão tiradas de estudos futuros. 


$$
\begin{gathered}
\mathcal{L}_{l_{1}, l_{2}, l_{3}}\left(n_{1} n_{2}, n_{0}\right)=\sqrt{\left(2 l_{1}+1\right)\left(2 l_{2}+1\right)\left(2 l_{3}+1\right)}\left(\left(\begin{array}{ccc}
l_{1} & l_{2} & l_{3} \\
0 & 0 & 0
\end{array}\right) P_{l_{1}}^{0}\left(z_{1}\right) P_{l_{2}}^{0}\left(z_{2}\right)\right. \\
+\sum_{m=1}^{m i n\left(l_{1}, l_{2}\right)}\left(\begin{array}{ccc}
l_{1} & l_{2} & l_{3} \\
m & -m & 0
\end{array}\right) \sqrt{\frac{\left(l_{1}-m\right) !\left(l_{2}-m\right) !}{\left(l_{1}+m\right) !\left(l_{2}+m\right) !}} P_{l_{1}}^{m}\left(z_{1}\right) P_{l_{2}}^{m}\left(z_{2}\right) \\
\left.(-1)^{m}\left[(A-i B)^{m}+(-1)^{l_{1}+l_{2}+l_{3}}(A+i B)^{m}\right]\right) .
\end{gathered}
$$

1) $\underline{\mathcal{L}_{0,0,0}\left(\mathbf{n}_{1}, \mathbf{n}_{2}, \mathbf{n}_{\mathbf{0}}\right) \text { : }}$

$$
\left(\begin{array}{lll}
0 & 0 & 0 \\
0 & 0 & 0
\end{array}\right) P_{0}^{0}\left(z_{1}\right) P_{0}^{0}(0)=1
$$

2) $\underline{\mathcal{L}_{0,1,1}\left(\mathbf{n}_{1}, \mathbf{n}_{2}, \mathbf{n}_{\mathbf{0}}\right):}$

$$
\begin{gathered}
\sqrt{(2.0+1)(2.1+1)(2.1+1)}\left(\begin{array}{ccc}
0 & 1 & 1 \\
0 & 0 & 0
\end{array}\right) P_{0}^{0}\left(z_{1}\right) P_{1}^{0}\left(z_{2}\right) \\
=\frac{-3}{\sqrt{3}} z_{2}=-\sqrt{3}<n_{0}, n_{2}>.
\end{gathered}
$$


As outras triplas $(1,0,1)$ e $(1,1,0)$ são cálculadas fazendo uma permutação nos índices, ou seja, $\mathcal{L}_{1,0,1}\left(n_{1}, n_{2}, n_{0}\right)=-\sqrt{3}<n_{0}, n_{1}>$ e $\mathcal{L}_{1,1,0}\left(n_{1}, n_{2}, n_{0}\right)=-\sqrt{3}<$ $n_{1}, n_{2}>$

3) $\mathcal{L}_{1,1,1}\left(\mathbf{n}_{1}, \mathbf{n}_{2}, \mathbf{n}_{\mathbf{0}}\right)$ :

$$
\begin{gathered}
\sqrt{(2.1+1)(2.1+1)(2.1+1)}\left(\left(\begin{array}{lll}
1 & 1 & 1 \\
0 & 0 & 0
\end{array}\right) P_{1}^{0}\left(z_{1}\right) P_{1}^{0}\left(z_{2}\right)+\frac{1}{2}\left(\begin{array}{ccc}
1 & 1 & 1 \\
1 & -1 & 0
\end{array}\right)(-1)(-2 i B)\right) \\
=\frac{1}{2 \sqrt{6}}(2 i B)=\frac{3}{\sqrt{2}} i B
\end{gathered}
$$

4) $\underline{\mathcal{L}_{1,1,2}\left(\mathbf{n}_{1}, \mathbf{n}_{2}, \mathbf{n}_{\mathbf{0}}\right) \text { : }}$

$$
\begin{gathered}
\sqrt{(2.1+1)(2.1+1)(2.2+1)}\left(\left(\begin{array}{lll}
1 & 1 & 2 \\
0 & 0 & 0
\end{array}\right) P_{1}^{0}\left(z_{1}\right) P_{1}^{0}\left(z_{2}\right)+\frac{1}{2}\left(\begin{array}{ccc}
1 & 1 & 2 \\
1 & -1 & 0
\end{array}\right)(-1)(2 A)\right) \\
=\sqrt{\frac{3}{2}}\left(z_{1}\left(3 z_{2}^{2}-1\right)+3 z_{2} A\right)=\frac{3 \sqrt{2}}{2 \sqrt{3}}\left(3<n_{0}, n_{1}><n_{0}, n_{2}>-<n_{1}, n_{2}>\right) .
\end{gathered}
$$

As outras triplas $(1,2,1)$ e $(2,1,1)$ são cálculadas fazendo uma permutação nos índices, ou seja, $\mathcal{L}_{1,2,1}\left(n_{1}, n_{2}, n_{0}\right)=\frac{3 \sqrt{2}}{2 \sqrt{3}}\left(3<n_{0}, n_{2}><n_{1}, n_{2}>-<n_{0}, n_{1}>\right)$ e $\mathcal{L}_{2,1,1}\left(n_{1}, n_{2}, n_{0}\right)=\frac{3 \sqrt{2}}{2 \sqrt{3}}\left(3<n_{0}, n_{1}><n_{1}, n_{2}>-<n_{0}, n_{2}>\right)$.

5) $\underline{\mathcal{L}_{1,2,2}\left(\mathbf{n}_{1}, \mathbf{n}_{2}, \mathbf{n}_{0}\right) \text { : }}$

$$
\begin{gathered}
\sqrt{(2.1+1)(2.2+1)(2.2+1)}\left(\left(\begin{array}{ccc}
1 & 2 & 2 \\
0 & 0 & 0
\end{array}\right) P_{1}^{0}\left(z_{1}\right) P_{2}^{0}\left(z_{2}\right)+\frac{3 z_{2}}{2 \sqrt{3}}\left(\begin{array}{ccc}
1 & 2 & 2 \\
1 & -1 & 0
\end{array}\right)(-1)(-2 i B)\right) \\
\left.=-\frac{15 \sqrt{2}}{2 \sqrt{5}} i B<n_{0}, n_{2}\right\rangle .
\end{gathered}
$$

As outras triplas $(2,1,2)$ e $(2,2,1)$ são cálculadas fazendo uma permutação nos índices, ou seja, $\mathcal{L}_{2,1,2}\left(n_{1}, n_{2}, n_{0}\right)=-\frac{15 \sqrt{2}}{2 \sqrt{5}} i B<n_{0}, n_{1}>$ e $\mathcal{L}_{2,2,1}\left(n_{1}, n_{2}, n_{0}\right)=-\frac{15 \sqrt{2}}{2 \sqrt{5}} i B<$ $n_{1}, n_{2}>$. 
6) $\mathcal{L}_{\mathbf{0 , 2 , 2}}\left(\mathbf{n}_{1}, \mathbf{n}_{\mathbf{2}}, \mathbf{n}_{\mathbf{0}}\right)$ :

$$
\begin{gathered}
\sqrt{(2.0+1)(2.2+1)(2.2+1)}\left(\left(\begin{array}{ccc}
0 & 2 & 2 \\
0 & 0 & 0
\end{array}\right) P_{0}^{0}\left(z_{1}\right) P_{2}^{0}\left(z_{2}\right)\right. \\
=5 \frac{1}{2 \sqrt{5}}\left(3 z_{1}^{2}-1\right)=\frac{\sqrt{5}}{2}\left(3<n_{0}, n_{2}>^{2}-1\right) .
\end{gathered}
$$

As outras triplas $(2,0,2)$ e $(2,2,0)$ são cálculadas fazendo uma permutação nos índices, ou seja, $\mathcal{L}_{2,0,2}\left(n_{1}, n_{2}, n_{0}\right)=\frac{\sqrt{5}}{2}\left(3<n_{0}, n_{1}>^{2}-1\right)$ e $\mathcal{L}_{2,2,0}\left(n_{1}, n_{2}, n_{0}\right)=\frac{\sqrt{5}}{2}(3<$ $\left.n_{1}, n_{2}>^{2}-1\right)$.

7) $\mathcal{L}_{2,2,2}\left(\mathbf{n}_{1}, \mathbf{n}_{2}, \mathbf{n}_{0}\right)$ :

$$
\begin{gathered}
\sqrt{(2.1+1)(2.2+1)(2.2+1)}\left(\left(\begin{array}{ccc}
2 & 2 & 2 \\
0 & 0 & 0
\end{array}\right) P_{2}^{0}\left(z_{1}\right) P_{2}^{0}\left(z_{2}\right)+\frac{9}{24}\left(\begin{array}{ccc}
2 & 2 & 2 \\
2 & -2 & 0
\end{array}\right) 2\left(A^{2}-B^{2}\right)\right. \\
\left.+\frac{9 z_{1} z_{2}}{6}\left(\begin{array}{ccc}
2 & 2 & 2 \\
1 & -1 & 0
\end{array}\right)(-1)(2 A)\right)=\frac{-5 \sqrt{2}}{4 \sqrt{7}}\left(\left(3 z_{1}^{2}-1\right)\left(3 z_{2}^{2}-1\right)-3\left(A^{2}-B^{2}\right)+6 z_{1} z_{2} A\right) \\
=\frac{-5 \sqrt{2}}{2 \sqrt{7}}\left(3 B^{2}-1+3<n_{0}, n_{1}><n_{0}, n_{2}><n_{1}, n_{2}>\right),
\end{gathered}
$$

usando a propriedade $2<n_{0}, n_{1}><n_{0}, n_{2}><n_{1}, n_{2}>=R+B^{2}-1$, obtemos:

$$
=\frac{-5 \sqrt{2}}{4 \sqrt{7}}\left(9 B^{2}+3 R-5\right)
$$

8) $\mathcal{L}_{\mathbf{0 , 3 , 3}}\left(\mathbf{n}_{\mathbf{1}}, \mathbf{n}_{\mathbf{2}}, \mathbf{n}_{\mathbf{0}}\right)$ :

$$
\begin{gathered}
\sqrt{(2.0+1)(2.3+1)(2.3+1)}\left(\left(\begin{array}{ccc}
0 & 3 & 3 \\
0 & 0 & 0
\end{array}\right) P_{0}^{0}\left(z_{1}\right) P_{3}^{0}\left(z_{2}\right)=\frac{-7}{2 \sqrt{7}} z_{2}\left(5 z_{2}^{2}-3\right)\right. \\
=\frac{-7}{2 \sqrt{7}}<n_{0}, n_{2}>\left(5<n_{0}, n_{2}>^{2}-3\right) .
\end{gathered}
$$

para as outras triplas basta fazer uma permutação nos índices.

9) $\underline{\mathcal{L}_{1,2,3}\left(\mathbf{n}_{1}, \mathbf{n}_{2}, \mathbf{n}_{\mathbf{0}}\right) \text { : }}$ 


$$
\begin{gathered}
\sqrt{(2.1+1)(2.2+1)(2.3+1)}\left(\left(\begin{array}{ccc}
1 & 2 & 3 \\
0 & 0 & 0
\end{array}\right) P_{1}^{0}\left(z_{1}\right) P_{2}^{0}\left(z_{2}\right)\right. \\
\left.-\sqrt{\frac{0 ! 1 !}{2 ! 3 !}} \frac{[2 A] P_{1}^{1}\left(z_{1}\right) P_{2}^{1}\left(z_{2}\right)}{\left(1-z_{1}^{2}\right)^{1 / 2}\left(1-z_{2}\right)^{1 / 2}}\left(\begin{array}{ccc}
1 & 2 & 3 \\
1 & -1 & 0
\end{array}\right)\right) \\
=\sqrt{105}\left\{\frac{-1}{2} \sqrt{\frac{3}{25}} z_{1}\left(3 z_{2}^{2}-1\right)+\frac{3 A z_{2}}{\sqrt{105}}\right\} \\
=\frac{3}{2}\left(2<n_{0}, n_{2}><n_{1}, n_{2}>+<n_{0}, n_{1}>-5<n_{0}, n_{1}><n_{0}, n_{2}>^{2}\right) .
\end{gathered}
$$

E para $\mathcal{L}_{3,1,2}\left(n_{1}, n_{2}, n_{0}\right), \mathcal{L}_{2,3,1}\left(n_{1}, n_{2}, n_{0}\right), \mathcal{L}_{2,1,3}\left(n_{1}, n_{2}, n_{0}\right), \mathcal{L}_{1,3,2}\left(n_{1}, n_{2}, n_{0}\right)$ e $\mathcal{L}_{3,2,1}\left(n_{1}, n_{2}, n_{0}\right)$ basta fazer a ciclicidade dos índices.

10) $\mathcal{L}_{\mathbf{2 , 2}, 3}\left(\mathbf{n}_{1}, \mathbf{n}_{2}, \mathbf{n}_{\mathbf{0}}\right)$ :

$$
\begin{gathered}
\sqrt{(2.2+1)(2.2+1)(2.3+1)}\left(\left(\begin{array}{ccc}
2 & 2 & 3 \\
0 & 0 & 0
\end{array}\right) P_{2}^{0}\left(z_{1}\right) P_{2}^{0}\left(z_{2}\right)+\frac{1 !}{3 !} \frac{[2 i B] P_{2}^{1}\left(z_{1}\right) P_{2}^{1}\left(z_{2}\right)}{\left(1-z_{1}^{2}\right)^{1 / 2}\left(1-z_{2}^{2}\right)^{1 / 2}}\left(\begin{array}{ccc}
2 & 2 & 3 \\
1 & -1 & 0
\end{array}\right)\right. \\
\left.-\frac{0 !}{4 !} \frac{[4 i A B] P_{2}^{2}\left(z_{1}\right) P_{2}^{2}\left(z_{2}\right)}{\left(1-z_{1}^{2}\right)\left(1-z_{2}^{2}\right)}\left(\begin{array}{ccc}
2 & 2 & 3 \\
2 & -2 & 0
\end{array}\right)\right) \\
=5 \sqrt{7}\left\{3 \sqrt{\frac{2}{35}} i B z_{1} z_{2}-\frac{3 i A B}{\sqrt{70}}\right\} \\
=15 \sqrt{\frac{2}{5}} i B\left(5<n_{0}, n_{1}><n_{0}, n_{2}>-<n_{1}, n_{2}>\right) .
\end{gathered}
$$

E por permutação dos índices obtemos $\mathcal{L}_{3,2,2}\left(n_{1}, n_{2}, n_{0}\right)$ e $\mathcal{L}_{2,3,2}\left(n_{1}, n_{2}, n_{0}\right)$.

11) $\underline{\mathcal{L}_{1,3,3}\left(\mathbf{n}_{1}, \mathbf{n}_{2}, \mathbf{n}_{\mathbf{0}}\right):}$

$$
\begin{aligned}
\sqrt{(2.1+1)(2.3+1)(2.3+1)} & \left.\left(\begin{array}{ccc}
1 & 3 & 3 \\
0 & 0 & 0
\end{array}\right) P_{1}^{0}\left(z_{1}\right) P_{3}^{0}\left(z_{2}\right)+\sqrt{\frac{1 ! 2 !}{2 ! 4 !}} \frac{[2 i B] P_{1}^{1}\left(z_{1}\right) P_{3}^{1}\left(z_{2}\right)}{\left(1-z_{1}^{2}\right)^{1 / 2}\left(1-z_{2}^{2}\right)^{1 / 2}}\left(\begin{array}{ccc}
1 & 3 & 3 \\
1 & -1 & 0
\end{array}\right)\right) \\
& =7 \sqrt{3}\left(-\frac{3}{4 \sqrt{21}} i B\left(1-5 z_{2}^{2}\right)\right) \\
& =-\frac{21}{4 \sqrt{7}} i B\left(1-<n_{0}, n_{2}>^{2}\right) .
\end{aligned}
$$


E para obter $\mathcal{L}_{3,3,1}\left(n_{1}, n_{2}, n_{0}\right)$ e $\mathcal{L}_{3,1,3}\left(n_{1}, n_{2}, n_{0}\right)$ basta fazermos uma permutação cíclica dos índices.

12) $\mathcal{L}_{\mathbf{2 , 3}, \mathbf{3}}\left(\mathbf{n}_{\mathbf{1}}, \mathbf{n}_{\mathbf{2}}, \mathbf{n}_{\mathbf{0}}\right)$ :

$$
\begin{gathered}
\sqrt{(2.2+1)(2.3+1)(2.3+1)}\left(\left(\begin{array}{ccc}
2 & 3 & 3 \\
0 & 0 & 0
\end{array}\right) P_{2}^{0}\left(z_{1}\right) P_{3}^{0}\left(z_{2}\right)\right. \\
-\sqrt{\frac{1 ! 2 !}{3 ! 4 !}} \frac{[2 A] P_{2}^{1}\left(z_{1}\right) P_{3}^{1}\left(z_{2}\right)}{\left(1-z_{1}^{2}\right)^{1 / 2}\left(1-z_{2}^{2}\right)^{1 / 2}}\left(\begin{array}{ccc}
2 & 3 & 3 \\
1 & -1 & 0
\end{array}\right) \\
\left.+\sqrt{\frac{0 ! 1 !}{4 ! 5 !}} \frac{P_{2}^{2}\left(z_{1}\right) P_{3}^{2}\left(z_{2}\right)}{\left(1-z_{1}^{2}\right)\left(1-z_{2}^{2}\right)}\left(\begin{array}{ccc}
2 & 3 & 3 \\
2 & -2 & 0
\end{array}\right) 2\left(A^{2}-B^{2}\right)\right) \\
=\frac{7}{4 \sqrt{21}}\left(2 z_{2}\left(5 z_{2}^{2}-3\right)\left(3 z_{1}^{2}-1\right)-3 A z_{1}\left(1-5 z_{2}^{2}\right)-15 z_{2}\left(A^{2}-B^{2}\right)\right) \\
=\frac{7}{4 \sqrt{21}}\left(-10<n_{0}, n_{2}>^{3}-15<n_{0}, n_{1}>^{2}<n_{0}, n_{2}>\right. \\
-15<n_{0}, n_{2}><n_{1}, n_{2}>^{2}+6<n_{0}, n_{2}>-3<n_{0}, n_{1}><n_{1}, n_{2}> \\
\left.+45<n_{0}, n_{1}><n_{0}, n_{2}>^{2}<n_{1}, n_{2}>+15 B^{2}<n_{0}, n_{2}>\right) .
\end{gathered}
$$

E por permutação cíclica dos índices obtemos $\mathcal{L}_{3,2,3}\left(n_{1}, n_{2}, n_{0}\right)$ e $\mathcal{L}_{3,3,2}\left(n_{1}, n_{2}, n_{0}\right)$.

13) $\mathcal{L}_{\mathbf{3 , 3}, \mathbf{3}}\left(\mathbf{n}_{\mathbf{1}}, \mathbf{n}_{\mathbf{2}}, \mathbf{n}_{\mathbf{0}}\right)$ :

$$
\begin{gathered}
\sqrt{(2.3+1)(2.3+1)(2.3+1)}\left(\left(\begin{array}{ccc}
3 & 3 & 3 \\
0 & 0 & 0
\end{array}\right) P_{3}^{0}\left(z_{1}\right) P_{3}^{0}\left(z_{2}\right)\right. \\
+\frac{2 !}{4 !} \frac{[2 i B] P_{3}^{1}\left(z_{1}\right) P_{3}^{1}\left(z_{2}\right)}{\left(1-z_{1}^{2}\right)^{1 / 2}\left(1-z_{2}^{2}\right)^{1 / 2}}\left(\begin{array}{ccc}
3 & 3 & 3 \\
1 & -1 & 0
\end{array}\right) \\
\left.\quad-\frac{1 !}{5 !} \frac{[4 i A B] P_{3}^{2}\left(z_{1}\right) P_{3}^{2}\left(z_{2}\right)}{\left(1-z_{1}^{2}\right)\left(1-z_{2}^{2}\right)}\left(\begin{array}{ccc}
3 & 3 & 3 \\
2 & -2 & 0
\end{array}\right)\right) \\
=\frac{7}{8 \sqrt{14}} i B\left(-3\left(1-5 z_{1}^{2}\right)\left(1-5 z_{2}^{2}\right)+60 A z_{1} z_{2}+15 A^{2}-5 B^{2}\right) \\
=\frac{35}{8 \sqrt{14}} i B\left(3\left(<n_{0}, n_{1}>^{2}+<n_{0}, n_{2}>^{2}+<n_{1}, n_{2}>^{2}\right)\right. \\
\left.-18<n_{0}, n_{1}><n_{0}, n_{2}><n_{1}, n_{2}>-B^{2}-3 / 5\right) .
\end{gathered}
$$


Onde aparece $<n_{0}, n_{1}><n_{0}, n_{2}><n_{1}, n_{2}>$, usamos a seguinte propriedade:

$$
1-B^{2}=R-2<n_{0}, n_{1}><n_{0}, n_{2}><n_{1}, n_{2}>,
$$

com a intensão de deixar as contas em função de produtos internos mais simples e determinante.

A soma em $l_{k}$ para $j=3 / 2$ foi feita da seguinte forma:

$$
\begin{aligned}
& L_{1}^{3 / 2}\left(n_{1}, n_{2}, n_{0}\right)=\frac{-2}{(4 \pi)^{2}}\left\{\left\{\begin{array}{ccc}
0 & 0 & 0 \\
3 / 2 & 3 / 2 & 3 / 2
\end{array}\right\} \mathcal{L}_{0,0,0}\left(n_{1}, n_{2}, n_{0}\right)+\left\{\begin{array}{ccc}
1 & 1 & 1 \\
3 / 2 & 3 / 2 & 3 / 2
\end{array}\right\} \mathcal{L}_{1,1,1}\left(n_{1}, n_{2}, n_{0}\right)\right. \\
& +\left\{\begin{array}{ccc}
0 & 1 & 1 \\
3 / 2 & 3 / 2 & 3 / 2
\end{array}\right\}\left(\mathcal{L}_{0,1,1}\left(n_{1}, n_{2}, n_{0}\right)+\mathcal{L}_{1,0,1}\left(n_{1}, n_{2}, n_{0}\right)+\mathcal{L}_{1,1,0}\left(n_{1}, n_{2}, n_{0}\right)\right) \\
& +\left\{\begin{array}{ccc}
1 & 1 & 2 \\
3 / 2 & 3 / 2 & 3 / 2
\end{array}\right\}\left(\mathcal{L}_{1,1,2}\left(n_{1}, n_{2}, n_{0}\right)+\mathcal{L}_{2,1,1}\left(n_{1}, n_{2}, n_{0}\right)+\mathcal{L}_{1,2,1}\left(n_{1}, n_{2}, n_{0}\right)\right) \\
& +\left\{\begin{array}{ccc}
1 & 2 & 2 \\
3 / 2 & 3 / 2 & 3 / 2
\end{array}\right\}\left(\mathcal{L}_{1,2,2}\left(n_{1}, n_{2}, n_{0}\right)+\mathcal{L}_{2,1,2}\left(n_{1}, n_{2}, n_{0}\right)+\mathcal{L}_{2,2,1}\left(n_{1}, n_{2}, n_{0}\right)\right) \\
& +\left\{\begin{array}{ccc}
1 & 2 & 3 \\
3 / 2 & 3 / 2 & 3 / 2
\end{array}\right\}\left(\mathcal{L}_{1,2,3}\left(n_{1}, n_{2}, n_{0}\right)+\mathcal{L}_{3,1,2}\left(n_{1}, n_{2}, n_{0}\right)+\mathcal{L}_{2,3,1}\left(n_{1}, n_{2}, n_{0}\right)\right. \\
& \left.+\mathcal{L}_{3,2,1}\left(n_{1}, n_{2}, n_{0}\right)+\mathcal{L}_{1,3,2}\left(n_{1}, n_{2}, n_{0}\right)+\mathcal{L}_{2,1,3}\left(n_{1}, n_{2}, n_{0}\right)\right) \\
& +\left\{\begin{array}{ccc}
1 & 3 & 3 \\
3 / 2 & 3 / 2 & 3 / 2
\end{array}\right\}\left(\mathcal{L}_{1,3,3}\left(n_{1}, n_{2}, n_{0}\right)+\mathcal{L}_{3,1,3}\left(n_{1}, n_{2}, n_{0}\right)+\mathcal{L}_{3,3,1}\left(n_{1}, n_{2}, n_{0}\right)\right) \\
& \left\{\begin{array}{ccc}
0 & 2 & 2 \\
3 / 2 & 3 / 2 & 3 / 2
\end{array}\right\}\left(\mathcal{L}_{0,2,2}\left(n_{1}, n_{2}, n_{0}\right)+\mathcal{L}_{2,0,2}\left(n_{1}, n_{2}, n_{0}\right)+\mathcal{L}_{2,2,0}\left(n_{1}, n_{2}, n_{0}\right)\right)
\end{aligned}
$$




$$
\begin{aligned}
& +\left\{\begin{array}{ccc}
0 & 3 & 3 \\
3 / 2 & 3 / 2 & 3 / 2
\end{array}\right\}\left(\mathcal{L}_{0,3,3}\left(n_{1}, n_{2}, n_{0}\right)+\mathcal{L}_{3,0,3}\left(n_{1}, n_{2}, n_{0}\right)+\mathcal{L}_{3,3,0}\left(n_{1}, n_{2}, n_{0}\right)\right) \\
& +\left\{\begin{array}{ccc}
2 & 2 & 3 \\
3 / 2 & 3 / 2 & 3 / 2
\end{array}\right\}\left(\mathcal{L}_{2,2,3}\left(n_{1}, n_{2}, n_{0}\right)+\mathcal{L}_{3,2,2}\left(n_{1}, n_{2}, n_{0}\right)+\mathcal{L}_{2,3,2}\left(n_{1}, n_{2}, n_{0}\right)\right) \\
& +\left\{\begin{array}{ccc}
2 & 3 & 3 \\
3 / 2 & 3 / 2 & 3 / 2
\end{array}\right\}\left(\mathcal{L}_{2,3,3}\left(n_{1}, n_{2}, n_{0}\right)+\mathcal{L}_{3,2,3}\left(n_{1}, n_{2}, n_{0}\right)+\mathcal{L}_{3,3,2}\left(n_{1}, n_{2}, n_{0}\right)\right) \\
& \left.+\left\{\begin{array}{ccc}
2 & 2 & 2 \\
3 / 2 & 3 / 2 & 3 / 2
\end{array}\right\} \mathcal{L}_{2,2,2}\left(n_{1}, n_{2}, n_{0}\right)+\left\{\begin{array}{ccc}
3 & 3 & 3 \\
3 / 2 & 3 / 2 & 3 / 2
\end{array}\right\} \mathcal{L}_{3,3,3}\left(n_{1}, n_{2}, n_{0}\right)\right]
\end{aligned}
$$

14) $\underline{\mathcal{L}_{\mathbf{0 , 4}, 4}\left(\mathbf{n}_{1}, \mathbf{n}_{2}, \mathbf{n}_{\mathbf{0}}\right) \text { : }}$

$$
\begin{gathered}
\sqrt{(2.0+1)(2.4+1)(2.4+1)}\left(\left(\begin{array}{ccc}
0 & 4 & 4 \\
0 & 0 & 0
\end{array}\right) P_{0}^{0}\left(z_{1}\right) P_{4}^{0}\left(z_{2}\right)\right. \\
=\frac{3}{8}\left(3-30 z_{2}^{2}+35 z_{2}^{4}\right)=\frac{3}{8}\left(3-30<n_{0}, n_{2}>^{2}+35<n_{0}, n_{2}>^{4}\right) .
\end{gathered}
$$

Por ciclicidade obtemos $\mathcal{L}_{4,0,4}\left(n_{1}, n_{2}, n_{0}\right)$ e $\mathcal{L}_{4,4,0}\left(n_{1}, n_{2}, n_{0}\right)$.

15) $\mathcal{L}_{\mathbf{1 , 3 , 4}}\left(\mathbf{n}_{1}, \mathbf{n}_{\mathbf{2}}, \mathbf{n}_{\mathbf{0}}\right)$ :

$$
\begin{gathered}
\sqrt{(2.1+1)(2.3+1)(2.4+1)}\left(\left(\begin{array}{ccc}
1 & 3 & 4 \\
0 & 0 & 0
\end{array}\right) P_{1}^{0}\left(z_{1}\right) P_{3}^{0}\left(z_{2}\right)+\right. \\
-\sqrt{\frac{0 ! 2 !}{2 ! 4 !}} \frac{[2 A] P_{1}^{1}\left(z_{1}\right) P_{3}^{1}\left(z_{2}\right)}{\left(1-z_{1}^{2}\right)^{1 / 2}\left(1-z_{2}^{2}\right)^{1 / 2}}\left(\begin{array}{ccc}
1 & 3 & 4 \\
1 & -1 & 0
\end{array}\right) \\
=\frac{\sqrt{27}}{12}\left(4 z_{1} z_{2}\left(5 z_{2}^{2}-3\right)+3 A\left(1-5 z_{2}^{2}\right)\right) \\
=\frac{\sqrt{27}}{12}\left(3<n_{1}, n_{2}>-15<n_{0}, n_{1}><n_{0}, n_{2}>+35<n_{0}, n_{2}>^{3}<n_{0}, n_{1}>\right.
\end{gathered}
$$




$$
\left.-15<n_{0}, n_{2}>^{2}<n_{1}, n_{2}>\right)
$$

Por ciclicidade obtemos $\mathcal{L}_{4,1,3}\left(n_{1}, n_{2}, n_{0}\right), \mathcal{L}_{3,4,1}\left(n_{1}, n_{2}, n_{0}\right), \mathcal{L}_{4,3,1}\left(n_{1}, n_{2}, n_{0}\right), \mathcal{L}_{1,4,3}\left(n_{1}, n_{2}, n_{0}\right)$ e $\mathcal{L}_{3,1,4}\left(n_{1}, n_{2}, n_{0}\right)$.

16) $\underline{\mathcal{L}_{1,4,4}\left(\mathbf{n}_{1}, \mathbf{n}_{2}, \mathbf{n}_{\mathbf{0}}\right):}$

$$
\begin{gathered}
\sqrt{(2.1+1)(2.4+1)(2.4+1)}\left(\left(\begin{array}{ccc}
1 & 4 & 4 \\
0 & 0 & 0
\end{array}\right) P_{1}^{0}\left(z_{1}\right) P_{4}^{0}\left(z_{2}\right)+\right. \\
+\sqrt{\frac{0 ! 3 !}{2 ! 5 !}} \frac{[2 i B] P_{1}^{1}\left(z_{1}\right) P_{4}^{1}\left(z_{2}\right)}{\left(1-z_{1}^{2}\right)^{1 / 2}\left(1-z_{2}^{2}\right)^{1 / 2}}\left(\begin{array}{ccc}
1 & 4 & 4 \\
1 & -1 & 0
\end{array}\right) \\
\left.=\frac{15 \sqrt{3}}{4 \sqrt{5}} i B\left(7 z_{2}^{3}-3 z_{2}\right)\right) \\
=\frac{15 \sqrt{3}}{4 \sqrt{5}} i B\left(7<n_{0}, n_{2}>^{3}-3<n_{0}, n_{2}>\right) .
\end{gathered}
$$

Por ciclicidade obtemos $\mathcal{L}_{4,1,4}\left(n_{1}, n_{2}, n_{0}\right)$ e $\mathcal{L}_{4,4,1}\left(n_{1}, n_{2}, n_{0}\right)$

17) $\mathcal{L}_{2,4,4}\left(\mathbf{n}_{1}, \mathbf{n}_{2}, \mathbf{n}_{\mathbf{0}}\right)$ :

$$
\begin{aligned}
& \sqrt{(2.2+1)(2.4+1)(2.4+1)}\left(\left(\begin{array}{ccc}
2 & 4 & 4 \\
0 & 0 & 0
\end{array}\right) P_{2}^{0}\left(z_{1}\right) P_{4}^{0}\left(z_{2}\right)+\right. \\
& -\sqrt{\frac{1 ! 3 !}{3 ! 5 !}} \frac{[2 A] P_{2}^{1}\left(z_{1}\right) P_{4}^{1}\left(z_{2}\right)}{\left(1-z_{1}^{2}\right)^{1 / 2}\left(1-z_{2}^{2}\right)^{1 / 2}}\left(\begin{array}{ccc}
2 & 4 & 4 \\
1 & -1 & 0
\end{array}\right) \\
& +\sqrt{\frac{0 ! 2 !}{4 ! 6 !}} \frac{\left[2\left(A^{2}-B^{2}\right)\right] P_{2}^{2}\left(z_{1}\right) P_{4}^{2}\left(z_{2}\right)}{\left(1-z_{1}^{2}\right)\left(1-z_{2}^{2}\right)}\left(\begin{array}{ccc}
2 & 4 & 4 \\
2 & -2 & 0
\end{array}\right) \\
& =\frac{15}{8 \sqrt{77}}\left(\left(1-3 z_{1}^{2}\right)\left(3-30 z_{2}^{2}+35 z_{2}^{4}\right)+6 A z_{1}\left(3 z_{2}-7 z_{2}^{3}\right)+9\left(7 z_{2}^{2}-1\right)\left(A^{2}-B^{2}\right)\right) \\
& =\frac{1}{8} \sqrt{\frac{5}{77}}\left(1-10<n_{0}, n_{2}>^{2}-3<n_{0}, n_{1}>^{2}+\frac{35}{3}<n_{0}, n_{2}>^{4}+21<n_{1}, n_{2}>^{2}<n_{0}, n_{2}>^{2}\right. \\
& +12<n_{0}, n_{1}><n_{0}, n_{2}><n_{1}, n_{2}>-21 B^{2}<n_{0}, n_{2}>^{2}+3 B^{2}
\end{aligned}
$$




$$
\left.+21<n_{0}, n_{1}>^{2}<n_{0}, n_{2}>^{2}-56<n_{0}, n_{1}><n_{0}, n_{2}>^{3}<n_{1}, n_{2}>-3<n_{1}, n_{2}>^{2}\right)
$$

Por ciclicidade obtemos $\mathcal{L}_{4,2,4}\left(n_{1}, n_{2}, n_{0}\right)$ e $\mathcal{L}_{4,4,2}\left(n_{1}, n_{2}, n_{0}\right)$.

18) $\mathcal{L}_{2,2,4}\left(\mathbf{n}_{1}, \mathbf{n}_{2}, \mathbf{n}_{\mathbf{0}}\right)$ :

$$
\begin{gathered}
\sqrt{(2.2+1)(2.2+1)(2.4+1)}\left(\left(\begin{array}{lll}
2 & 2 & 4 \\
0 & 0 & 0
\end{array}\right) P_{2}^{0}\left(z_{1}\right) P_{2}^{0}\left(z_{2}\right)+\right. \\
-\frac{1 !}{3 !} \frac{[2 A] P_{2}^{1}\left(z_{1}\right) P_{2}^{1}\left(z_{2}\right)}{\left(1-z_{1}^{2}\right)^{1 / 2}\left(1-z_{2}^{2}\right)^{1 / 2}}\left(\begin{array}{ccc}
2 & 2 & 4 \\
1 & -1 & 0
\end{array}\right) \\
+\frac{0 !}{4 !} \frac{\left[2\left(A^{2}-B^{2}\right)\right] P_{2}^{2}\left(z_{1}\right) P_{2}^{2}\left(z_{2}\right)}{\left(1-z_{1}^{2}\right)\left(1-z_{2}^{2}\right)}\left(\begin{array}{ccc}
2 & 4 & 4 \\
2 & -2 & 0
\end{array}\right) \\
=\frac{5}{8} \sqrt{\frac{18}{35}}\left(2\left(3 z_{1}^{2}-1\right)\left(3 z_{2}^{2}-1\right)-16 A z_{1} z_{2}+\left(A^{2}-B^{2}\right)\right) \\
=\frac{5}{8} \sqrt{\frac{18}{35}}\left(2-6<n_{0}, n_{1}>^{2}-6<n_{0}, n_{2}>^{2}+<n_{1}, n_{2}>^{2}\right. \\
\left.-18<n_{0}, n_{1}><n_{0}, n_{2}><n_{1}, n_{2}>+35<n_{0}, n_{1}>^{2}<n_{0}, n_{2}>^{2}-B^{2}\right) \\
\left.-8<n_{1}, n_{2}>^{2}-15<n_{0}, n_{2}>^{2}-15<n_{0}, n_{1}>^{2}\right) . \\
=\frac{5}{8} \sqrt{\frac{18}{35}}\left(11-10 B^{2}+35<n_{0}, n_{1}>^{2}<n_{0}, n_{2}>^{2}\right. \\
-8
\end{gathered}
$$

Por ciclicidade obtemos $\mathcal{L}_{4,2,2}\left(n_{1}, n_{2}, n_{0}\right)$ e $\mathcal{L}_{2,4,2}\left(n_{1}, n_{2}, n_{0}\right)$.

19) $\mathcal{L}_{\mathbf{2}, \mathbf{3}, \mathbf{4}}\left(\mathbf{n}_{\mathbf{1}}, \mathbf{n}_{\mathbf{2}}, \mathbf{n}_{\mathbf{0}}\right)$ :

$$
\begin{gathered}
\sqrt{(2.2+1)(2.3+1)(2.4+1)}\left(\left(\begin{array}{ccc}
2 & 3 & 4 \\
0 & 0 & 0
\end{array}\right) P_{2}^{0}\left(z_{1}\right) P_{3}^{0}\left(z_{2}\right)+\right. \\
+\sqrt{\frac{1 ! 2 !}{3 ! 4 !}} \frac{[2 i B] P_{2}^{1}\left(z_{1}\right) P_{3}^{1}\left(z_{2}\right)}{\left(1-z_{1}^{2}\right)^{1 / 2}\left(1-z_{2}^{2}\right)^{1 / 2}}\left(\begin{array}{ccc}
2 & 3 & 4 \\
1 & -1 & 0
\end{array}\right)
\end{gathered}
$$




$$
\begin{gathered}
-\sqrt{\frac{0 ! 1 !}{4 ! 5 !}} \frac{[4 i A B)] P_{2}^{2}\left(z_{1}\right) P_{3}^{2}\left(z_{2}\right)}{\left(1-z_{1}^{2}\right)\left(1-z_{2}^{2}\right)}\left(\begin{array}{ccc}
2 & 3 & 4 \\
2 & -2 & 0
\end{array}\right) \\
=\frac{5 \sqrt{9}}{4} i B\left(z_{1}\left(1-5 z_{2}^{2}\right)+2 A z_{2}\right) \\
=\frac{5 \sqrt{9}}{4} i B\left(<n_{0}, n_{1}>-7<n_{0}, n_{1}><n_{0}, n_{2}>^{2}+2<n_{0}, n_{2}><n_{1}, n_{2}>\right) .
\end{gathered}
$$

Por ciclicidade obtemos $\mathcal{L}_{4,2,3}\left(n_{1}, n_{2}, n_{0}\right), \mathcal{L}_{3,4,2}\left(n_{1}, n_{2}, n_{0}\right), \mathcal{L}_{4,3,2}\left(n_{1}, n_{2}, n_{0}\right), \mathcal{L}_{2,4,3}\left(n_{1}, n_{2}, n_{0}\right)$ e $\mathcal{L}_{3,2,4}\left(n_{1}, n_{2}, n_{0}\right)$.

20) $\mathcal{L}_{\mathbf{3 , 3}, 4}\left(\mathbf{n}_{1}, \mathbf{n}_{\mathbf{2}}, \mathbf{n}_{\mathbf{0}}\right)$ :

$$
\begin{gathered}
\sqrt{(2.3+1)(2.3+1)(2.4+1)}\left(\left(\begin{array}{lll}
3 & 3 & 4 \\
0 & 0 & 0
\end{array}\right) P_{3}^{0}\left(z_{1}\right) P_{3}^{0}\left(z_{2}\right)+\right. \\
-\frac{2 !}{4 !} \frac{[2 A] P_{3}^{1}\left(z_{1}\right) P_{3}^{1}\left(z_{2}\right)}{\left(1-z_{1}^{2}\right)^{1 / 2}\left(1-z_{2}^{2}\right)^{1 / 2}}\left(\begin{array}{ccc}
3 & 3 & 4 \\
1 & -1 & 0
\end{array}\right) \\
+\frac{1 !}{5 !} \frac{\left.\left[2\left(A^{2}-B^{2}\right)\right)\right] P_{3}^{2}\left(z_{1}\right) P_{3}^{2}\left(z_{2}\right)}{\left(1-z_{1}^{2}\right)\left(1-z_{2}^{2}\right)}\left(\begin{array}{ccc}
3 & 3 & 4 \\
2 & -2 & 0
\end{array}\right) \\
+\frac{0 !}{6 !} \frac{\left[2 A^{3}-6 A B^{2}\right] P_{3}^{3}\left(z_{1}\right) P_{3}^{3}\left(z_{2}\right)}{\left(1-z_{1}^{2}\right)^{3 / 2}\left(1-z_{2}^{2}\right)^{3 / 2}}\left(\begin{array}{ccc}
3 & 3 & 4 \\
3 & -3 & 0
\end{array}\right) \\
=\frac{21}{8 \sqrt{154}\left(-4 z_{1} z_{2}\left(5 z_{1}^{2}-3\right)\left(5 z_{2}^{2}-3\right)-A\left(1-5 z_{1}^{2}\right)\left(1-5 z_{2}^{2}\right)+70 z_{1} z_{2}\left(A^{2}-B^{2}\right)+5\left(A^{3}-3 A B^{2}\right)\right)} \\
=\frac{105}{8 \sqrt{154}\left(-2<n_{0}, n_{1}>^{3}<n_{0}, n_{2}>^{3}+11<n_{0}, n_{1}>^{3}<n_{0}, n_{2}>+11<n_{0}, n_{1}><n_{0}, n_{2}>^{3}\right.} \\
-30<n_{0}, n_{1}>^{2}<n_{0}, n_{2}>^{2}<n_{1}, n_{2}>-11 B^{2}<n_{0}, n_{1}><n_{0}, n_{2}> \\
-7<n_{0}, n_{1}><n_{0}, n_{2}>-\frac{1}{5}<n_{1}, n_{2}>+<n_{0}, n_{1}>^{2}<n_{1}, n_{2}>+<n_{0}, n_{2}>^{2}<n_{1}, n_{2}> \\
-30
\end{gathered}
$$




$$
\begin{gathered}
\left.+11<n_{0}, n_{1}><n_{0}, n_{2}><n_{1}, n_{2}>^{2}+<n_{1}, n_{2}>^{3}-3 B^{2}<n_{1}, n_{2}>\right) \\
=\frac{105}{8 \sqrt{154}}\left(-2<n_{0}, n_{1}>^{3}<n_{0}, n_{2}>^{3}-4<n_{0}, n_{1}>^{3}<n_{0}, n_{2}>-4<n_{0}, n_{1}><n_{0}, n_{2}>^{3}\right. \\
+8<n_{0}, n_{1}><n_{0}, n_{2}>-\frac{57}{10}<n_{1}, n_{2}>+\frac{13}{2}<n_{0}, n_{1}>^{2}<n_{1}, n_{2}>+\frac{13}{2}<n_{0}, n_{2}>^{2}<n_{1}, n_{2}> \\
-26 B^{2}<n_{0}, n_{1}><n_{0}, n_{2}>+\frac{13}{2}<n_{1}, n_{2}>^{3}-\frac{5}{2} B 2<n_{1}, n_{2}> \\
\left.-15<n_{1}, n_{2}>^{2}<n_{0}, n_{1}><n_{0}, n_{2}>\right) .
\end{gathered}
$$

Por ciclicidade obtemos $\mathcal{L}_{4,3,3}\left(n_{1}, n_{2}, n_{0}\right)$ e $\mathcal{L}_{3,4,3}\left(n_{1}, n_{2}, n_{0}\right)$.

21) $\mathcal{L}_{3,4,4}\left(\mathbf{n}_{1}, \mathbf{n}_{2}, \mathbf{n}_{\mathbf{0}}\right)$ :

$$
\begin{aligned}
& \sqrt{(2.3+1)(2.4+1)(2.4+1)}\left(\left(\begin{array}{ccc}
3 & 4 & 4 \\
0 & 0 & 0
\end{array}\right) P_{3}^{0}\left(z_{1}\right) P_{4}^{0}\left(z_{2}\right)+\right. \\
& +\sqrt{\frac{2 ! 3 !}{4 ! 5 !}} \frac{[2 i B] P_{3}^{1}\left(z_{1}\right) P_{4}^{1}\left(z_{2}\right)}{\left(1-z_{1}^{2}\right)^{1 / 2}\left(1-z_{2}^{2}\right)^{1 / 2}}\left(\begin{array}{ccc}
3 & 4 & 4 \\
1 & -1 & 0
\end{array}\right) \\
& -\sqrt{\frac{1 ! 2 !}{5 ! 6 !}} \frac{[4 i A B] P_{3}^{2}\left(z_{1}\right) P_{4}^{2}\left(z_{2}\right)}{\left(1-z_{1}^{2}\right)\left(1-z_{2}^{2}\right)}\left(\begin{array}{ccc}
3 & 4 & 4 \\
2 & -2 & 0
\end{array}\right) \\
& -\sqrt{\frac{0 ! 1 !}{6 ! 7 !}} \frac{\left[2 i B^{3}-6 i A^{2} B\right] P_{3}^{3}\left(z_{1}\right) P_{4}^{3}\left(z_{2}\right)}{\left(1-z_{1}^{2}\right)^{3 / 2}\left(1-z_{2}^{2}\right)^{3 / 2}}\left(\begin{array}{ccc}
3 & 4 & 4 \\
3 & -3 & 0
\end{array}\right) \\
& =\frac{1}{8} \sqrt{\frac{5}{154}} i B\left(-9\left(1-5 z_{1}^{2}\right)\left(7 z_{2}^{3}-3 z_{2}\right)+30 A z_{1}\left(7 z_{2}^{2}-1\right)+35 z_{2}\left(B^{2}-3 A^{2}\right)\right) \\
& =\frac{1}{8} \sqrt{\frac{5}{154}} i B\left(-21<n_{0}, n_{2}>^{3}+9<n_{0}, n_{2}>\right.
\end{aligned}
$$


$+140<n_{1}, n_{2}><n_{0}, n_{1}><n_{0}, n_{2}>^{2}-10<n_{0}, n_{1}><n_{1}, n_{2}>-35<n_{0}, n_{1}>^{2}<n_{0}, n_{2}>$

$$
\left.+\frac{35}{3} B^{2}<n_{0}, n_{2}>-35<n_{0}, n_{2}><n_{1}, n_{2}>^{2}\right) .
$$

Por ciclicidade obtemos $\mathcal{L}_{4,3,4}\left(n_{1}, n_{2}, n_{0}\right)$ e $\mathcal{L}_{4,4,3}\left(n_{1}, n_{2}, n_{0}\right)$.

22) $\mathcal{L}_{4,4,4}\left(\mathbf{n}_{1}, \mathbf{n}_{2}, \mathbf{n}_{\mathbf{0}}\right):$

$$
\begin{aligned}
& \sqrt{(2.4+1)(2.4+1)(2.4+1)}\left(\left(\begin{array}{ccc}
4 & 4 & 4 \\
0 & 0 & 0
\end{array}\right) P_{4}^{0}\left(z_{1}\right) P_{4}^{0}\left(z_{2}\right)+\right. \\
& +\sum_{m=1}^{4} \frac{(4-m) !}{(4+m) !} \frac{P_{4}^{m}\left(z_{1}\right) P_{4}^{m}\left(z_{2}\right)}{\left(1-z_{1}^{2}\right)^{m / 2}\left(1-z_{2}^{2}\right)^{m / 2}}\left(\begin{array}{ccc}
4 & 4 & 4 \\
m & -m & 0
\end{array}\right) \\
& \cdot(-1)^{m}\left[(A-i B)^{m}+(A+i B)^{m}\right] \\
& =\frac{5}{96 \sqrt{2002}}\left(\frac{81}{5}-22<n_{1}, n_{2}>^{2}+49<n_{1}, n_{2}>^{4}+22 B^{2}-294<n_{1}, n_{2}>^{2} B^{2}\right. \\
& +49 B^{4}-162<n_{0}, n_{1}>^{2}+154<n_{1}, n_{2}>^{2}<n_{0}, n_{1}>^{2}-154 B^{2}<n_{0}, n_{1}>^{2} \\
& +189<n_{0}, n_{1}>^{4}+368<n_{1}, n_{2}><n_{0}, n_{1}><n_{0}, n_{2}> \\
& -784<n_{1}, n_{2}>^{3}<n_{0}, n_{1}><n_{0}, n_{2}>+2352 B^{2}<n_{1}, n_{2}><n_{0}, n_{1}><n_{0}, n_{2}> \\
& -1064<n_{1}, n_{2}><n_{0}, n_{1}>^{3}<n_{0}, n_{2}>-162<n_{0}, n_{2}>^{2}+154<n_{1}, n_{2}>^{2}<n_{0}, n_{2}>^{2} \\
& -154 B^{2}<n_{0}, n_{2}>^{2}+1274<n_{0}, n_{1}>^{2}<n_{0}, n_{2}>^{2}+980<n_{1}, n_{2}>^{2}<n_{0}, n_{1}>^{2}<n_{0}, n_{2}>^{2}
\end{aligned}
$$




$$
\begin{gathered}
-980 B^{2}<n_{0}, n_{1}>^{2}<n_{0}, n_{2}>^{2}-980<n_{0}, n_{1}>^{4}<n_{0}, n_{2}>^{2} \\
-1064<n_{1}, n_{2}><n_{0}, n_{1}><n_{0}, n_{2}>^{3}+1960<n_{1}, n_{2}><n_{0}, n_{1}>^{3}<n_{0}, n_{2}>^{3} \\
\left.+189<n_{0}, n_{2}>^{4}-980<n_{0}, n_{1}>^{2}<n_{0}, n_{2}>^{4}\right) \\
=\frac{5}{96 \sqrt{2002}}\left(\frac{1611}{5}+1715 B^{4}-1950 B^{2}-426 R+1470 R B^{2}+105 R^{2}+42 Q\right),
\end{gathered}
$$

onde $Q=<n_{0}, n_{1}>^{4}+<n_{0}, n_{2}>^{4}+<n_{1}, n_{2}>^{4}$ e usamos novamente que $2<n_{1}, n_{2}><n_{0}, n_{1}><n_{0}, n_{2}>=B^{2}+R-1$.

Lista dos polinômios associados de Legendre, $P_{l}^{m}(z)$, usados em todos os cálculos dos $\underline{\mathcal{L}_{l_{1}, l_{2}, l_{3}}\left(n_{1}, n_{2}, n_{0}\right) \text { acima: }}$

$$
\begin{gathered}
P_{0}^{0}(z)=1, \quad P_{1}^{0}(z)=z, \quad P_{1}^{1}(z)=-\left(1-z^{2}\right)^{1 / 2}, \quad P_{2}^{0}(z)=\frac{1}{2}\left(3 z^{2}-1\right)^{1 / 2}, \\
P_{2}^{1}(z)=-3 z\left(1-z^{2}\right)^{1 / 2}, \quad P_{2}^{2}(z)=3\left(1-z^{2}\right), \quad P_{3}^{0}(z)=\frac{1}{2} z\left(5 z^{2}-3\right), \\
P_{3}^{1}(z)=\frac{3}{2}\left(1-5 z^{2}\right)\left(1-z^{2}\right)^{1 / 2}, \quad P_{3}^{2}(z)=15 z\left(1-z^{2}\right)^{1 / 2}, \quad P_{3}^{3}(z)=-15\left(1-z^{2}\right)^{3 / 2}, \\
P_{4}^{0}(z)=\frac{1}{8}\left(3-30 z^{2}+35 z^{4}\right)^{3 / 2}, \quad P_{4}^{1}(z)=-\frac{5}{2}\left(1-z^{2}\right)^{1 / 2}\left(7 z^{3}-3 z\right), \quad P_{4}^{2}(z)=\frac{15}{2}\left(1-z^{2}\right)\left(7 z^{2}-1\right), \\
P_{4}^{3}(z)=-105\left(1-z^{2}\right)^{3 / 2}, \quad P_{4}^{4}(z)=105\left(1-z^{2}\right)^{2} .
\end{gathered}
$$





\section{Apêndice B: Tetraedro}

A seguir seguem os dados e as fórmulas usadas no capitúlo 3, maior detalhes ver [17].

Considere um tetraedro de vértices 1,2, 3 e 4, tais que:

vértices $1,2,3$ cuja face (oposta ao vértice 4$)$ delimitada pelas arestas $(j, j \lambda, j)$;

vértices 1,2, 4 cuja face (oposta ao vértice 3 ) delimitada pelas arestas $\left(j, j \lambda_{2}, j\right)$;

vértices 1, 3, 4 cuja face (oposta ao vértice 2) delimitada pelas arestas $\left(j, \lambda_{1}, j\right)$;

vértices $2,3,4$ cuja face (oposta ao vértice 1 ) delimitada pelas arestas $\left(j \lambda_{2}, j \lambda_{1}, j \lambda\right)$.

Seja $A_{h}$ a área da face oposta ao vértice $h$.

O volume do tetraedro é dado por, [17]

$$
V^{2}=\frac{j^{6}}{144} \Delta
$$

onde $\Delta=\left(\lambda_{1}+\lambda_{2}+\lambda\right)\left(-\lambda_{1}+\lambda_{2}+\lambda\right)\left(\lambda_{1}-\lambda_{2}+\lambda\right)\left(\lambda_{1}+\lambda_{2}-\lambda\right)$

Lembrando que $\theta_{\lambda_{i}}$ para $i=1,2,3,4$, são os ângulos diedrais, ou seja, o ângulo formado pela intersecção das duas faces de aresta comum $j \lambda_{i}$.

$$
A_{h} A_{k} \operatorname{sen}\left(\theta_{\lambda_{h k}}\right)=\frac{3}{2} V j_{h k}
$$

onde $h, k$ são os vértices e $j_{h k}$ a aresta de vértices $h$ e $k$.

No caso do tetraedro em questão: $j_{12}=j_{13}=j_{14}=j, j_{24}=j \lambda_{2}, j_{23}=j \lambda$ e $j_{34}=j \lambda_{1}$. A partir dessas informações vamos calcular inicialmente a área das faces $A_{h}$ para $h=1,2,3,4$.

$$
A_{1}=\frac{j^{2}}{4} \sqrt{\Delta} ;
$$




$$
\begin{aligned}
& A_{2}=\frac{j^{2} \lambda_{1}}{4} \sqrt{\left(4-\lambda_{1}^{2}\right)} \\
& A_{3}=\frac{j^{2} \lambda_{2}}{4} \sqrt{\left(4-\lambda_{2}^{2}\right)} \\
& A_{4}=\frac{j^{2} \lambda}{4} \sqrt{\left(4-\lambda^{2}\right)}
\end{aligned}
$$

Agora o cálculo dos $\theta_{h k}$ :

$$
\begin{gathered}
\operatorname{sen}\left(\theta_{12}\right)=\frac{3 V j_{12}}{2 A_{1} A_{2}}=\frac{2 \sqrt{\left(\Delta-\lambda_{1}^{2} \lambda_{2}^{2} \lambda^{2}\right)}}{\sqrt{\Delta\left(4-\lambda_{1}^{2}\right)}} ; \\
\operatorname{sen}\left(\theta_{13}\right)=\frac{3 V j_{13}}{2 A_{1} A_{3}}=\frac{2 \sqrt{\left(\Delta-\lambda_{1}^{2} \lambda_{2}^{2} \lambda^{2}\right)}}{\sqrt{\Delta\left(4-\lambda_{2}^{2}\right)}} ; \\
\operatorname{sen}\left(\theta_{14}\right)=\frac{3 V j_{14}}{2 A_{1} A_{4}}=\frac{2 \sqrt{\left(\Delta-\lambda_{1}^{2} \lambda_{2}^{2} \lambda^{2}\right)}}{\sqrt{\Delta\left(4-\lambda^{2}\right)}} \\
\operatorname{sen}\left(\theta_{34}\right)=\frac{3 V j_{34}}{2 A_{3} A_{4}}=\frac{2 \sqrt{\left(\Delta-\lambda_{1}^{2} \lambda_{2}^{2} \lambda^{2}\right)}}{\lambda_{2} \lambda \sqrt{\left(4-\lambda_{2}^{2}\right)\left(4-\lambda^{2}\right)}} \\
\operatorname{sen}\left(\theta_{24}\right)=\frac{3 V j_{24}}{2 A_{2} A_{4}}=\frac{2 \sqrt{\left(\Delta-\lambda_{1}^{2} \lambda_{2}^{2} \lambda^{2}\right)}}{\lambda_{1} \lambda \sqrt{\left(4-\lambda_{1}^{2}\right)\left(4-\lambda^{2}\right)}} \\
\operatorname{sen}\left(\theta_{23}\right)=\frac{3 V j_{23}}{2 A_{2} A_{3}}=\frac{2 \sqrt{\left(\Delta-\lambda_{1}^{2} \lambda_{2}^{2} \lambda^{2}\right)}}{\lambda_{1} \lambda_{2} \sqrt{\left(4-\lambda_{1}^{2}\right)\left(4-\lambda_{2}^{2}\right)}}
\end{gathered}
$$

Agora o cálculo para os $\theta_{\lambda_{j}}, j=1,2,3$ e de $D, E$ para o $3 j-$ simbolo,

$$
\left(\begin{array}{ccc}
j \lambda_{1} & j \lambda_{2} & j \lambda \\
-j \mu_{1} & -j \mu_{2} & j m
\end{array}\right)
$$

segue:

$$
\begin{aligned}
& \cos \left(\theta_{\lambda_{1}}\right)=\frac{2 \lambda_{1}^{2} \mu-\mu_{1}\left(\lambda_{1}^{2}-\lambda_{2}^{2}+\lambda^{2}\right)}{\sqrt{\left(\lambda_{1}^{2}-\mu_{1}^{2}\right)\left(4 \lambda^{2} \lambda_{1}^{2}-\left(\lambda_{1}^{2}-\lambda_{2}^{2}+\lambda_{3}^{2}\right)^{2}\right)}} \\
& \cos \left(\theta_{\lambda_{2}}\right)=\frac{2 \lambda_{2}^{2} \mu_{1}-\mu_{2}\left(\lambda_{1}^{2}+\lambda_{2}^{2}-\lambda^{2}\right)}{\sqrt{\left(\lambda_{2}^{2}-\mu_{2}^{2}\right)\left(4 \lambda_{1}^{2} \lambda_{2}^{2}-\left(\lambda_{1}^{2}+\lambda_{2}^{2}-\lambda_{3}^{2}\right)^{2}\right)}}
\end{aligned}
$$




$$
\begin{gathered}
\cos \left(\theta_{\lambda}\right)=\frac{2 \lambda^{2} \mu_{2}+\mu\left(-\lambda_{1}^{2}+\lambda_{2}^{2}+\lambda^{2}\right)}{\sqrt{\left(\lambda_{3}^{2}-\mu_{3}^{2}\right)\left(4 \lambda_{2}^{2} \lambda^{2}-\left(-\lambda_{1}^{2}+\lambda_{2}^{2}+\lambda_{3}^{2}\right)^{2}\right)}} \\
\cos (D)=\frac{1}{2} \frac{\lambda_{1}^{2}-\lambda_{2}^{2}-\lambda^{2}+2 \mu_{2} \mu}{\sqrt{\left(\lambda_{2}^{2}-\mu_{2}^{2}\right)\left(\lambda^{2}-\mu^{2}\right)}} \\
\cos (E)=\frac{1}{2} \frac{-\lambda_{1}^{2}+\lambda_{2}^{2}-\lambda^{2}+2 \mu_{1} \mu}{\sqrt{\left(\lambda_{2}^{2}-\mu_{2}^{2}\right)\left(\lambda^{2}-\mu^{2}\right)}} \\
\cos (F)=\frac{1}{2} \frac{-\lambda_{1}^{2}-\lambda_{2}^{2}+\lambda^{2}+2 \mu_{1} \mu_{2}}{\sqrt{\left(\lambda_{1}^{2}-\mu_{1}^{2}\right)\left(\lambda_{2}^{2}-\mu_{2}^{2}\right)}}
\end{gathered}
$$

E obtendo a área $\mathfrak{A}$ que é calculada pela fórmula de Heron's, segundo o apendice $\mathrm{B}$ de [17], para o triangulo de vértices $a, b, c$ da seguinte maneira:

$$
\mathfrak{A}^{2}=\frac{1}{16}(a+b+c)(-a+b+c)(a-b+c)(a+b-c)=-\frac{1}{16}\left|\begin{array}{cccc}
0 & a^{2} & b^{2} & 1 \\
a^{2} & 0 & c^{2} & 1 \\
b^{2} & c^{2} & 0 & 1 \\
1 & 1 & 1 & 0
\end{array}\right|
$$

Logo, no caso onde $a=j \lambda_{1}, b=j \lambda_{2}$ e $c=j \lambda$, temos $\mathfrak{A}=\frac{j^{2}}{4} \sqrt{\widetilde{\Delta}}$, onde

$$
\begin{array}{r}
\widetilde{\Delta}=\left(\lambda_{1}^{2}-\mu_{1}^{2}\right)\left(-\lambda_{1}^{2}+\mu_{1}^{2}+\lambda_{2}^{2}-\mu_{2}^{2}+\lambda^{2}-\mu^{2}\right)+ \\
\left(\lambda_{2}^{2}-\mu_{2}^{2}\right)\left(\lambda_{1}^{2}-\mu_{1}^{2}-\lambda_{2}^{2}+\mu_{2}^{2}+\lambda^{2}-\mu^{2}\right)+ \\
\left(\lambda^{2}-\mu^{2}\right)\left(\lambda_{1}^{2}-\mu_{1}^{2}+\lambda_{2}^{2}-\mu_{2}^{2}-\lambda^{2}+\mu^{2}\right) .
\end{array}
$$



Nesse apêndice se encontram todos os passos da resolução do exemplo 3.1.4. No exemplo foi usado o método "Steepest descents". Para usar tal método foram necessárias algumas contas exaustivas para conseguir resolvê-lo e que iremos explicitá-las aqui.

\subsection{Cálculo da aproximação para o produto de Strato- novich}

Temos que a integral originária do produto de Stratonovich é a seguinte:

$$
\begin{gathered}
Y_{j \lambda_{1}}^{j \mu_{1}} *_{1} Y_{j \lambda_{2}}^{j \mu_{2}} \sim \\
\sim \frac{\widetilde{g}_{2}}{8 \pi} \int_{1 / 4}^{3 / 4} g_{1}\left(e^{i\left(\Sigma_{1}+\Sigma_{2}+\Sigma_{3}\right)}+e^{i\left(\Sigma_{1}+\Sigma_{2}-\Sigma_{3}\right)}+e^{i\left(\Sigma_{1}-\Sigma_{2}+\Sigma_{3}\right)}+e^{i\left(\Sigma_{1}-\Sigma_{2}-\Sigma_{3}\right)}+\right. \\
\left.e^{i\left(-\Sigma_{1}+\Sigma_{2}+\Sigma_{3}\right)}+e^{i\left(-\Sigma_{1}+\Sigma_{2}-\Sigma_{3}\right)}+e^{i\left(-\Sigma_{1}-\Sigma_{2}+\Sigma_{3}\right)}+e^{i\left(-\Sigma_{1}-\Sigma_{2}-\Sigma_{3}\right)}\right) d \lambda \\
=\frac{\widetilde{g}_{2}}{8 \pi}\left(P_{1}+P_{2}+P_{3}+P_{4}+P_{5}+P_{6}+P_{7}+P_{8}\right) d \lambda
\end{gathered}
$$

onde, no caso desse exemplo em que $\lambda_{1}=1 / 2$ e $\lambda_{2}=1 / 4$,

$$
\frac{\widetilde{g}_{2}}{8 \pi}=\frac{(-1)^{j \frac{7}{4}+\frac{1}{2}}}{2 \pi^{3}} .
$$


Iniciemos com a resolução de $P_{1}$, que é a primeira parcela dessa integral. Iremos sistematizar a resolução dessa primeira integral e as outras parcelas serão de resolução análoga. O método ${ }^{3}$ nos fornece uma aproximação para a integral do tipo $P_{1}(j)$, que em ordem mais baixa, é dada por:

$$
P_{1}(j)=\frac{\sqrt{2 \pi} g_{1}\left(z_{0}\right) e^{j i f\left(z_{0}\right)} e^{i \gamma\left[x_{0}\right]}}{\left|j f^{\prime \prime}\left(z_{0}\right)\right|^{1 / 2}},
$$

onde $z_{0}$ é o ponto crítico da função fase $f(\lambda)$ e $\gamma\left[x_{0}\right]$ faz o papel de " $\alpha$ "na descrição do método (ver 3.6). Para obtermos essa aproximação, vamos escrever a função fase na forma de $u(x, y)+i v(x, y)$. E aplicar o método.

Observação: aqui colocamos o "i"em evidência.

Vamos colocar a seguir uma tabela de valores que serão usados em todos os cálculos para encontrar a função fase, através das somas (ou subtrações) dos $\Sigma_{1}, \Sigma_{2}$ e $\Sigma_{3}$, lembrando que nesse exemplo de cálculo está sendo usado $\lambda_{1}=1 / 2$ e $\lambda_{2}=1 / 4$. Aqui também consideramos $\mu_{1}=\mu_{2}=0$ e $\vartheta=\pi / 2$.

E os $\theta_{r s}$ são,

$$
\theta_{12}=\operatorname{Arcsen}\left(h_{12}(\lambda)\right)
$$

onde $h_{12}(\lambda)=\frac{4}{\sqrt{15}} \sqrt{\frac{256 \lambda^{4}-156 \lambda^{2}+9}{256 \lambda^{4}-160 \lambda^{2}+9}}$

$$
\theta_{13}=\operatorname{Arcsen}\left(h_{13}(\lambda)\right)
$$

onde $h_{13}(\lambda)=\frac{8}{3 \sqrt{7}} \sqrt{\frac{256 \lambda^{4}-156 \lambda^{2}+9}{256 \lambda^{4}-160 \lambda^{2}+9}}$

$$
\theta_{14}=\operatorname{Arcsen}\left(h_{14}(\lambda)\right)
$$

onde $h_{14}(\lambda)=2 \sqrt{\frac{-256 \lambda^{4}+156 \lambda^{2}-9}{256 \lambda^{6}-1184 \lambda^{4}+649 \lambda^{2}-36}}$

$$
\theta_{34}=\operatorname{Arcsen}\left(h_{34}(\lambda)\right),
$$

onde $h_{34}(\lambda)=\frac{2}{3 \sqrt{7} \lambda} \sqrt{\frac{256 \lambda^{4}-156 \lambda^{2}+9}{\lambda^{2}-4}}$

$$
\theta_{24}=\operatorname{Arcsen}\left(h_{24}(\lambda)\right),
$$

onde $h_{24}(\lambda)=\frac{1}{2 \sqrt{15 \lambda}} \sqrt{\frac{256 \lambda^{4}-156 \lambda^{2}+9}{\lambda^{2}-4}}$

$$
\theta_{23}=\operatorname{Arcsen}\left(h_{23}(\lambda)\right),
$$

onde $h_{23}(\lambda)=\frac{8}{3 \sqrt{105}} \sqrt{-256 \lambda^{4}+156 \lambda^{2}-9}$.

\footnotetext{
${ }^{3}$ detalhamos o método na subseção 3.6
} 


$$
\theta_{\lambda_{1}}=\theta_{\lambda_{2}}=\theta_{\lambda}=\pi / 2
$$

Agora, enfim, começemos as contas. Inicialmente com $P_{1}$ :

$$
P_{1}(j)=\int_{1 / 4}^{3 / 4} g_{1} e^{i j f(\lambda)} d \lambda
$$

onde a $f(\lambda)$ é a função fase,

$$
f(\lambda)=\frac{1}{2} \theta_{12}+\frac{1}{4} \theta_{13}+\lambda \theta_{14}+\theta_{34}+\theta_{24}+\theta_{23}+\lambda \pi .
$$

Se plotarmos a função fase $f(\lambda)$ tal qual se apresenta acima, observaremos que o gráfico estará com "bicos", como pode-se ver na figura a seguir: 


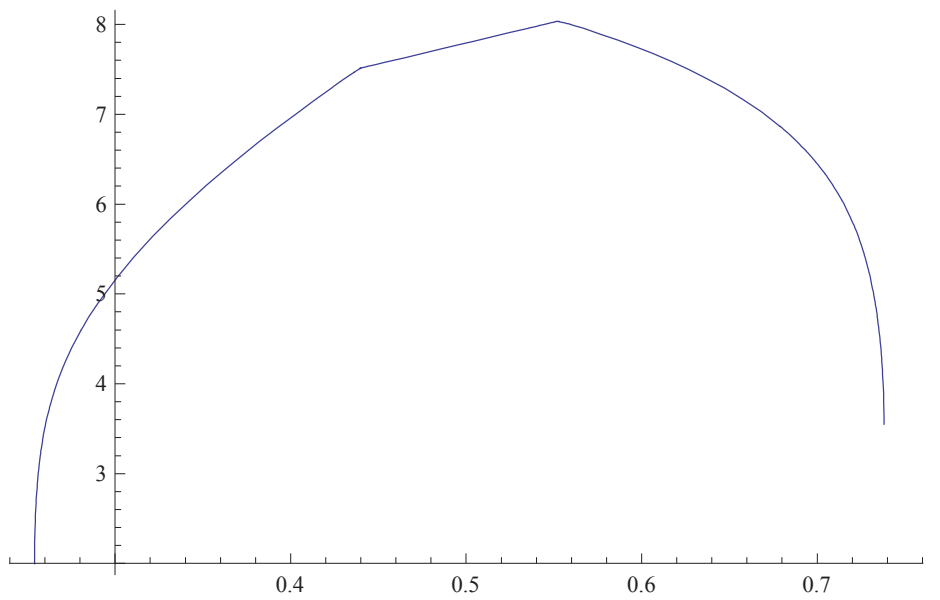


Para solucionar esse problema, redefiniremos a função $f(\lambda)$ da seguinte maneira:

$$
f(\lambda)=\left\{\begin{array}{l}
f_{1}(\lambda), \quad \text { se } \lambda \in[1 / 4, \sqrt{3} / 4], \\
f_{2}(\lambda), \quad \text { se } \lambda \in[\sqrt{3} / 4, \sqrt{6 / 31}], \\
f_{3}(\lambda), \quad \text { se } \lambda \in\left[\sqrt{6 / 31}, \frac{1}{8} \sqrt{39 / 2}\right], \\
f_{4}(\lambda), \quad \text { se } \lambda \in\left[\frac{1}{8} \sqrt{39 / 2}, \sqrt{5} / 4\right] \\
f_{5}(\lambda), \quad \text { se } \lambda \in[\sqrt{5} / 4,3 / 4]
\end{array}\right.
$$

onde

$$
\begin{gathered}
f_{1}(\lambda)=\frac{1}{2} \theta_{12}+\frac{1}{4} \theta_{13}+\lambda \theta_{14}+\theta_{34}+\theta_{24}+\theta_{23}+\frac{1}{2} \theta_{\lambda_{1}}+\frac{1}{4} \theta_{\lambda_{2}}+\lambda \theta_{\lambda}+\lambda \pi / 2, \\
f_{2}(\lambda)=\frac{1}{2}\left(\pi-\theta_{12}\right)+\frac{1}{4} \theta_{13}+\lambda \theta_{14}+\theta_{34}+\theta_{24}+\theta_{23}+\frac{1}{2} \theta_{\lambda_{1}}+\frac{1}{4} \theta_{\lambda_{2}}+\lambda \theta_{\lambda}+\lambda \pi / 2 \\
f_{3}(\lambda)=\frac{1}{2}\left(\pi-\theta_{12}\right)+\frac{1}{4} \theta_{13}+\lambda \theta_{14}+\left(\pi-\theta_{34}\right)+\theta_{24}+\theta_{23}+\frac{1}{2} \theta_{\lambda_{1}}+\frac{1}{4} \theta_{\lambda_{2}}+\lambda \theta_{\lambda}+\lambda \pi / 2 \\
f_{4}(\lambda)=\frac{1}{2}\left(\pi-\theta_{12}\right)+\frac{1}{4} \theta_{13}+\lambda \theta_{14}+\left(\pi-\theta_{34}\right)+\theta_{24}+\left(\pi-\theta_{23}\right)+\frac{1}{2} \theta_{\lambda_{1}}+\frac{1}{4} \theta_{\lambda_{2}}+\lambda \theta_{\lambda}+\lambda \pi / 2 \\
f_{5}(\lambda)=\frac{1}{2}\left(\pi-\theta_{12}\right)+\frac{1}{4} \theta_{13}+\lambda\left(\pi-\theta_{14}\right)+\left(\pi-\theta_{34}\right)+\theta_{24}+\left(\pi-\theta_{23}\right)+\frac{1}{2} \theta_{\lambda_{1}}+\frac{1}{4} \theta_{\lambda_{2}}+\lambda \theta_{\lambda}+\lambda \pi / 2 \\
\text { E fazendo isso o gráfico da função fase fica suave e claramente se vê um ponto de } \\
\text { inflexão em torno do número 0.5. Como podemos ver na figura a seguir: }
\end{gathered}
$$




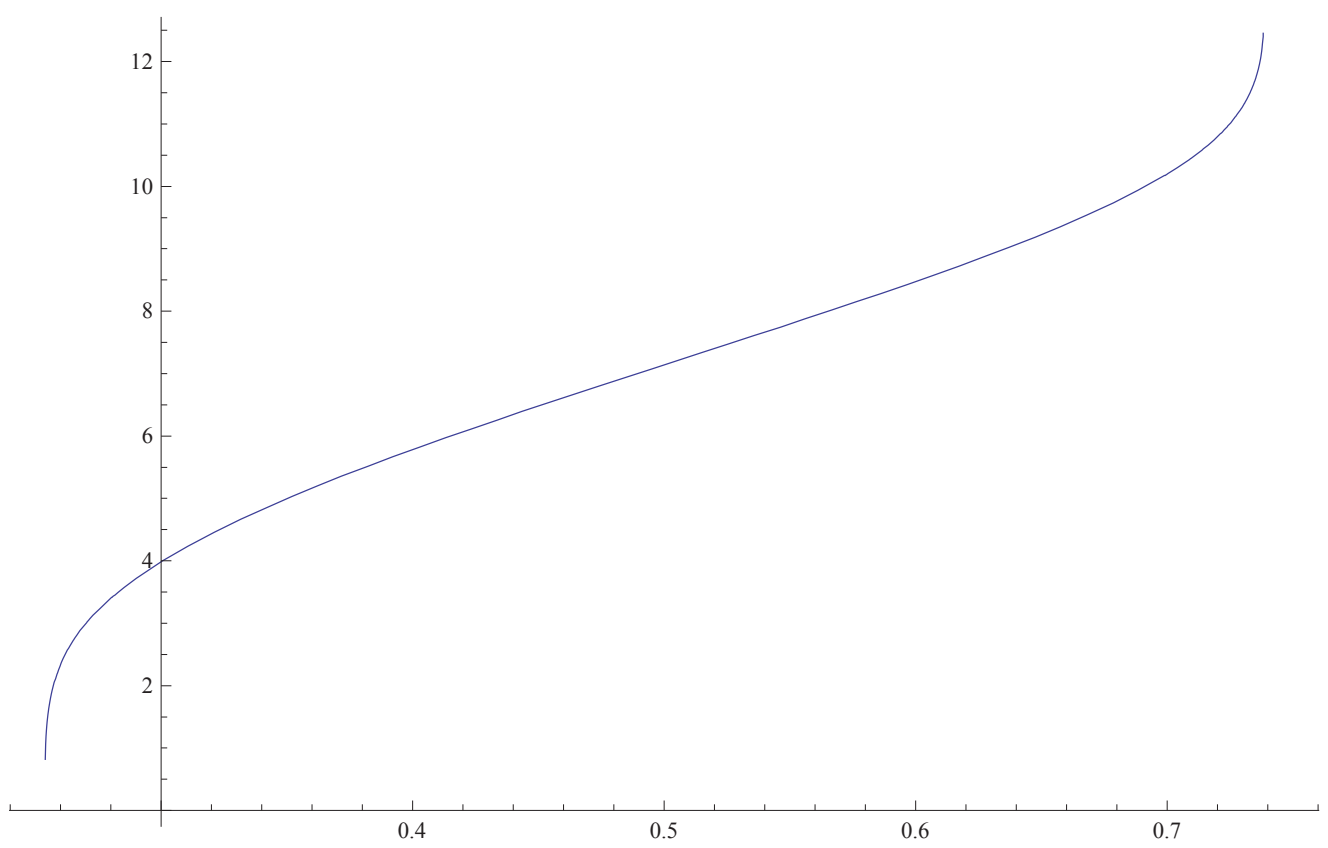


Para encontrar esse número, usaremos o mathematica Wolfram ${ }^{4}$, e através de aproximações para o encontro dessa raiz, encontramos $\widetilde{\lambda}_{1}=0.509677$ (ponto de inflexão) que se encontra no intervalo $\left[\sqrt{6 / 31}, \frac{1}{8} \sqrt{39 / 2}\right]$.

Fazendo a expansão em série de Taylor da $f_{3}(\lambda)$ (usamos a $f_{3}$ pois o ponto de inflexão está em seu domínio) em torno desse ponto e truncando até o grau 7, obtemos:

- Truncando em ordem 7 :

$$
\begin{aligned}
& \operatorname{pol}_{7}(\lambda)=7.26704+13.0451(-0.509677+\lambda)+37.0173(-0.509677+\lambda)^{3}+6.84793(-0.509677+\lambda)^{4} \\
& \quad+327.931(-0.509677+\lambda)^{5}+229.966(-0.509677+\lambda)^{6}+3754.07(-0.509677+l 3)^{7}
\end{aligned}
$$

O próximo passo é encontrar os candidatos a ponto de sela desse polinômio e trabalharemos com aqueles que deixam a parte imaginária de $\operatorname{pol}_{7}(\lambda)$ (ao fazer $\left.\lambda=x+i y\right)$ postiva.

Pontos de sela para $\mathrm{pol}_{7}$ :

$$
\begin{aligned}
& \lambda_{0}=0.291197+i 0.185923, \\
& \lambda_{1}=0.500299+i 0.287617, \\
& \lambda_{2}=0.711282+i 0.179422 .
\end{aligned}
$$

Agora, trocaremos $\lambda=x+i y$ para obter a extensão analítica

$$
f_{3}(\lambda) \sim \operatorname{pol}_{7}(x+i y)=u_{7}(x, y)+i v_{7}(x, y)
$$

onde

$$
\begin{gathered}
u_{7}(x, y)=u 7=-44.60853+562.10543 x-2958.75168 x^{2}+9132.44734 x^{3}-17329.02036 x^{4} \\
+20103.82749 x^{5}-13163.5797 x^{6}+3754.07097 x^{7}+2958.75168 y^{2}-27397.34202 x y^{2}+ \\
103974.12218 x^{2} y^{2}-201038.2748983257^{6} x^{3} y^{2}+197453.69555 x^{4} y^{2}-78835.49033 x^{5} y^{2} \\
-17329.02036 y^{4}+100519.13745 x y^{4}-197453.69555 x^{2} y^{4}+131392.48388 x^{3} y^{4} \\
+13163.5797 y^{6}-26278.49678 x y^{6}
\end{gathered}
$$

$\mathrm{e}$

$$
v_{7}(x, y)=562.10544 y-5917.50336 x y+27397.34202 x^{2} y-69316.08145 x^{3} y
$$

\footnotetext{
${ }^{4}$ olhar Apêndice D onde estão algumas das contas feitas no programa
} 


$$
\begin{gathered}
+100519.13745 x^{4} y-78981.47822 x^{5} y+26278.49678 x^{6} y-9132.44734 y^{3} \\
+69316.08145 x y^{3}-201038.2749 x^{2} y^{3}+263271.59406 x^{3} y^{3}-131392.48388 x^{4} y^{3} \\
+20103.82749 y^{5}-78981.47822 x y^{5}+78835.49033 x^{2} y^{5}-3754.07097 y^{7}
\end{gathered}
$$

Antes de finalizarmos as contas para essa parcela, vamos encontrar o valor de $\gamma\left[x_{0}\right]$ da fórmula usada no método de "steepest descents". A idéia é considerarmos a função $u_{7}(x, y)$ que é a parte imaginária da função fase (lembremos que $i$ está em evidência) e tomarmos a derivada em relação a $x$ igual a zero, $D_{x}[u 7]=0$. Para encontrarmos o valor de $\gamma\left[x_{0}\right]$, fazemos $x=x_{0}$, onde $x_{0}$ é a parte real do ponto de sela escolhido, em $D_{x}\left[u_{7}\right]=0$ e sabendo o valor de $y_{0}$, conseguimos obter o valor de $y^{\prime}\left[x_{0}\right]$, que denotamos por $\gamma\left[x_{0}\right]$. Fazendo essas contas, obtemos os resultados.

Os valores de $\gamma\left[x_{k}\right]=\gamma_{k}$ da fórmula de "steepest descents":

$$
\begin{gathered}
\gamma_{0} \approx 1.03 \\
\gamma_{1} \approx 24.2 \\
\gamma_{2} \approx 16 .
\end{gathered}
$$

Com todas as informações resta agora, analisarmos as contribuições de cada ponto de sela na expressão dada por 3.3.1 tanto para o polinômio de grau 7 como para a função fase original.

- $\operatorname{pol}_{7}(\lambda)$ em $\lambda_{0}$ :

$$
\begin{gathered}
P_{1}(j) \approx i e^{i j 3 \pi / 8} \frac{\sqrt{2 \pi}}{\sqrt{j} 2.40194}(2.22622-i 1.0929) e^{-i j \pi(-0.291197-i 0.185923)} e^{i j(5.22423+i 2.44792)} e^{-i 1.03} \\
\approx i \frac{1.044}{\sqrt{j}}(2.22622-i 1.0929) e^{j(-0.5841+i 0.9148)} e^{i j(6.40233+i 2.44792)} e^{-i 1.03} \\
\approx i \frac{1.044}{\sqrt{j}} e^{-i 1.03}(2.22622-i 1.0929) e^{j(-3.03202)} e^{i j 7.31713}
\end{gathered}
$$

- $\operatorname{pol}_{7}(\lambda)$ em $\lambda_{1}$ :

$$
\begin{gathered}
P_{1}(j) \approx i e^{i j 3 \pi / 8} \frac{\sqrt{2 \pi}}{\sqrt{j} 2.78294}(1.97798-i 0.060729) e^{-i j \pi(-0.500299-i 0.287617)} e^{i j(7.18304+i 2.89576)} e^{i 24.2} \\
\approx i \frac{0.9}{\sqrt{j}}(1.97798-i 0.060729) e^{j(-0.903575+i 1.57174)} e^{i j(8.36114+i 2.89576)} e^{i 24.2}
\end{gathered}
$$




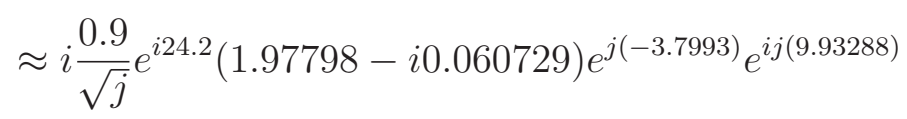

- $\operatorname{pol}_{7}(\lambda)$ em $\lambda_{2}$ :

$$
\begin{gathered}
P_{1}(j) \approx i e^{i j 3 \pi / 8} \frac{\sqrt{2 \pi}}{\sqrt{j} 3.06983}(2.11392+i 1.09795) e^{-i j \pi(-0.711282-i 0.179422)} e^{i j(9.14521+i 2.27478)} e^{i 16} \\
\approx i \frac{0.817}{\sqrt{j}}(2.11392+i 1.09795) e^{j(-0.56367+i 2.23456)} e^{i j(10.3233+i 2.27478)} e^{i 16} \\
\approx i \frac{0.817}{\sqrt{j}} e^{i 16}(2.11392+i 1.09795) e^{j(-2.83845)} e^{i j(12.55789)}
\end{gathered}
$$

Façamos agora para a próxima parcela $P_{2}$, e repitiremos a "receita"usada para encontrar $P_{1}$ :

$$
P_{2}(j)=\int_{1 / 4}^{3 / 4} g_{1} e^{i j f(\lambda)} d \lambda,
$$

onde a $f(\lambda)$ é a função fase,

$$
f(\lambda)=\frac{1}{2} \theta_{12}+\frac{1}{4} \theta_{13}+\lambda \theta_{14}+\theta_{34}+\theta_{24}+\theta_{23}
$$

Reescrevendo essa função fase usando a mesma idéia para a função fase da $P_{1}$, coincidentemente, encontramos o mesmo ponto de inflexão no mesmo intervalo. Porém a $f_{3}(\lambda)$ agora tem uma sutil diferença, não temos a parcela $\lambda \pi$.

$$
f_{3}(\lambda)=\frac{1}{2}\left(\pi-\theta_{12}\right)+\frac{1}{4} \theta_{13}+\lambda \theta_{14}+\pi-\theta_{34}+\theta_{24}+\theta_{23} .
$$

Tomamos seu polinômio de Taylor truncado em ordem 7:

- $\operatorname{pol}_{7}(\lambda)$ :

$$
\begin{gathered}
\operatorname{pol}_{7}(\lambda)=-44.6085+558.964 \lambda-2958.75 \lambda^{2}+9132.45 \lambda^{3}-17329 . \lambda^{4} \\
+20103.8 \lambda^{5}-13163.6 \lambda^{6}+3754.07 \lambda^{7}
\end{gathered}
$$

com os seguintes pontos de sela que nos interessam (lembrando que foram escolhidos justamente os que mantem a parte imaginária positiva);

$$
\begin{aligned}
& \lambda_{0}=0.303903+i 0.18464, \\
& \lambda_{1}=0.500573+i 0.27115,
\end{aligned}
$$




$$
\lambda_{2}=0.698302+i 0.177222
$$

Escrevendo $f_{3}(\lambda) \approx \operatorname{pol}_{7}(\lambda)=u_{7}(x, y)+i v_{7}(x, y)$, fazendo $\lambda=x+i y$, com

$$
\begin{gathered}
u_{7}(x, y)=-44.60853+558.96385 x-2958.75168 x^{2}+9132.44734 x^{3}-17329.02036 x^{4} \\
+20103.827489 x^{5}-13163.5797 x^{6}+3754.07097 x^{7}+2958.75168 y^{2}-27397.34202 x y^{2} \\
+103974.12218 x^{2} y^{2}-201038.2749 x^{3} y^{2}+197453.69555 x^{4} y^{2}-78835.49033 x^{5} y^{2} \\
-17329.020363 y^{4}+100519.13745 x y^{4}-197453.69555 x^{2} y^{4}+131392.48388 x^{3} y^{4} \\
+13163.5797 y^{6}-26278.49678 x\left(y^{6}\right)
\end{gathered}
$$

$$
v_{7}(x, y)=558.96385 y-5917.50336 x y+27397.34202 x^{2} y-69316.08145 x^{3} y
$$$$
+100519.13745 x^{4} y-78981.47822 x^{5} y+26278.49678 x^{6} y-9132.44734 y^{3}+69316.08145 x y^{3}
$$$$
-201038.2749 x^{2} y^{3}+263271.5941 x^{3} y^{3}-131392.48388 x^{4} y^{3}+20103.82749 y^{5}
$$

$$
-78981.47822 x y^{5}+78835.49033 x^{2} y^{5}-3754.07097 y^{7}
$$

Os valores de $\gamma\left[x_{k}\right]$, para $k=0,1,2$, da fórmula usada no método de "steepest descents", para essa parcela são dados por:

$$
\begin{gathered}
\gamma_{0} \approx 0.167, \pi \\
\gamma_{1} \approx 0.3, \\
\gamma_{2} \approx 0.14 .
\end{gathered}
$$

Fazendo os mesmos cálculos, obtemos a seguinte aproximação para essa integral, usando as contribuições dadas pelo polinômio de Taylor truncado de ordem 7 e usando 3.3.1:

- $\operatorname{pol}_{7}(\lambda)$ em $\lambda_{0}$ :

$$
\begin{aligned}
P_{2}(j) & \approx i e^{i j \frac{3 \pi}{8}} \frac{\sqrt{2 \pi}}{\sqrt{j} 14.7828}(2.27491-i 1.02783) e^{-i j \pi(-0.303903-i 0.18464)} e^{i j(4.29024+i 1.86602)} e^{i 1.67} \\
& \approx i \frac{0.16956}{\sqrt{j}}(2.27491-i 1.02783) e^{j(-0.5801+i 0.954739)} e^{i j(5.46834+i 0.250784)} e^{i 1.67}
\end{aligned}
$$




$$
\approx i \frac{0.16956}{\sqrt{j}} e^{i 1.67}(2.27491-i 1.02783) e^{j(-0.83088)} e^{i j(6.88342)}
$$

- $\operatorname{pol}_{7}(\lambda)$ em $\lambda_{1}$ :

$$
\begin{gathered}
P_{2}(j) \approx i e^{i j \frac{3 \pi}{8}} \frac{\sqrt{2 \pi}}{\sqrt{j} 12.7689}(2.04074-0.0546291 i) e^{-i j \pi(-0.500573-0.27115 i)} e^{i j(5.61094+i 2.01674)} e^{i \gamma_{0}} \\
\approx i \frac{0.196}{\sqrt{j}}(2.04074-0.0546291 i) e^{j(-0.851843+i 1.572597)} e^{i j(6.78904+i 2.01674)} e^{i 0.3} \\
\approx i \frac{0.196}{\sqrt{j}} e^{i 0.3}(2.04074-0.0546291 i) e^{j(-2.868583)} e^{i j(8.36201)}
\end{gathered}
$$

- $\operatorname{pol}_{7}(\lambda)$ em $\lambda_{2}$ :

$$
\begin{gathered}
P_{2}(j) \approx i e^{i j \frac{3 \pi}{8}} \frac{\sqrt{2 \pi}}{\sqrt{j} 14.5245}(2.18382+i 1.03107) e^{-i j \pi(-0.698302-i 0.177222)} e^{i j(6.93019+i 1.71464)} e^{i 0.14} \\
\approx i \frac{0.1726}{\sqrt{j}}(2.18382+i 1.03107) e^{j(-0.55676+i 2.19378)} e^{i j(6.93019+i 1.71464)} e^{i 0.14} \\
\approx i \frac{0.1726}{\sqrt{j}} e^{i 0.14}(2.18382+i 1.03107) e^{j(-2.2714)} e^{i j(9.12397)}
\end{gathered}
$$

Procedendo para $P_{3}$ :

$$
P_{3}(j)=e^{-3 \pi / 8} \int_{1 / 4}^{3 / 4} g_{1} e^{i j f(\lambda)} d \lambda
$$

onde a $f(\lambda)$ é a função fase,

$$
f(\lambda)=\frac{1}{2} \theta_{12}+\frac{1}{4} \theta_{13}+\lambda \theta_{14}+\theta_{34}+\theta_{24}+\theta_{23} .
$$

Tomamos seu polinômio de Taylor, truncado em ordem 7:

$\operatorname{pol}_{7}(\lambda)=-44.6085+558.964 \lambda-2958.75 \lambda^{2}+9132.45 \lambda^{3}-17329 \lambda^{4}+20103.8 \lambda^{5}-13163.6 \lambda^{6}+3754.07 \lambda^{7}$, com os seguinte pontos de sela que nos interessam:

$$
\begin{aligned}
& \lambda_{0}=0.303903+i 0.18464, \\
& \lambda_{1}=0.500573+i 0.27115, \\
& \lambda_{2}=0.698302+i 0.177222 .
\end{aligned}
$$


Observamos que a função fase é a mesma da $P_{2}$ a menos de uma constante $\frac{-j 3 \pi}{8}$.

Escrevendo $f(\lambda) \approx \operatorname{pol}_{7}(\lambda)=u_{7}(x, y)+i v_{7}(x, y)$, fazendo $\lambda=x+i y$, onde

$$
\begin{gathered}
u_{7}(x, y)=-44.60853+558.96384 x-2958.75168 x^{2}+9132.44734 x^{3}-17329.02036 x^{4} \\
+20103.82749 x^{5}-13163.5797 x^{6}+3754.07097 x^{7}+2958.75168 y^{2} \\
-27397.34202 x y^{2}+103974.1222 x^{2} y^{2}-201038.2749 x^{3} y^{2}+197453.695545 x^{4} y^{2} \\
-78835.49033 x^{5} y^{2}-17329.020363 y^{4}+100519.13745 x y^{4}-197453.69555 x^{2} y^{4} \\
+131392.48388 x^{3} y^{4}+13163.5797 y^{6}-26278.496775 x y^{6}
\end{gathered}
$$

e

$$
\begin{gathered}
v_{7}(x, y)=558.96385 y-5917.50336 x y+27397.34202 x^{2} y-69316.08145 x^{3} y \\
+100519.13745 x^{4} y-78981.47822 x^{5} y+26278.49678 x^{6} y-9132.44734 y^{3} \\
+69316.08145 x y^{3}-201038.2749 x^{2} y^{3}+263271.59406 x^{3} y^{3}-131392.48388 x^{4} y^{3} \\
+20103.82749 y^{5}-78981.47822 x y^{5}+78835.490323 x^{2} y^{5}-3754.070968 y^{7}
\end{gathered}
$$

Calculando os $\gamma\left[x_{k}\right]=\gamma_{k}$ da fórmula do método:

$$
\begin{aligned}
& \gamma_{0} \approx 3.33 \\
& \gamma_{1} \approx 1.89 \\
& \gamma_{2} \approx 0.56 .
\end{aligned}
$$

E as contribuições para cada caso:

- $\operatorname{pol}_{7}(\lambda)$ em $\lambda_{0}$ :

$$
\begin{gathered}
P_{3}(j) \approx e^{\frac{-i j 3 \pi}{8}} \frac{\sqrt{2 \pi}}{\sqrt{j} 14.7828}(2.27491-i 1.02783) e^{-i j \pi(-0.303903-i 0.18464)} e^{i j(4.29024+i 1.86602)} e^{i 3.33} \\
\approx \frac{0.169564}{\sqrt{j}}(2.27491-i 1.02783) e^{j(-0.58+i 0.95474)} e^{i j(3.11214+i 1.86602)} e^{i 3.33} \\
\approx \frac{0.169564}{\sqrt{j}} e^{i 3.33}(2.27491-i 1.02783) e^{j(-2.44602)} e^{i j(4.06688)}
\end{gathered}
$$

- $\operatorname{pol}_{7}(\lambda)$ em $\lambda_{1}$ :

$P_{3}(j) \approx e^{\frac{-i j 3 \pi}{8}} \frac{\sqrt{2 \pi}}{\sqrt{j} 12.7689}(2.04074-i 0.0546291) e^{-i j \pi(-0.500573-i 0.27115)} e^{i j(5.61094+i 2.01674)} e^{i 1.89}$ 


$$
\begin{gathered}
\approx \frac{0.2776}{\sqrt{j}}(2.04074-i 0.0546291) e^{j(-0.85184+i 1.572597)} e^{i j(4.43284+i 2.01674)} e^{i 1.89} \\
\approx \frac{0.2776}{\sqrt{j}} e^{i 1.89}(2.04074-i 0.0546291) e^{j(-2.86858)} e^{i j(6.01)}
\end{gathered}
$$

- $\operatorname{pol}_{7}(\lambda)$ em $\lambda_{2}$ :

$$
\begin{gathered}
P_{3}(j) \approx e^{\frac{-i j 3 \pi}{8}} \frac{\sqrt{2 \pi}}{\sqrt{j} 14.5245}(2.18382+i 1.03107) e^{-i j \pi(-0.698302-i 0.177222)} e^{i j(6.93019+i 1.71464)} e^{i 0.56} \\
\approx \frac{0.12204}{\sqrt{j}}(2.18382+i 1.03107) e^{j(-0.55676+i 2.19378)} e^{i j(5.75209+i 1.71464)} e^{i 0.56} \\
\approx \frac{0.12204}{\sqrt{j}} e^{i 0.56}(2.18382+i 1.03107) e^{j(-2.2714)} e^{i j(7.94587)}
\end{gathered}
$$

E por ultimo calculamos a aproximação para $P_{4}$ :

$$
P_{4}(j)=\int_{1 / 4}^{3 / 4} g_{1} e^{i j f(\lambda)} d \lambda,
$$

onde a $f(\lambda)$ é a função fase,

$$
f(\lambda)=\frac{1}{2} \theta_{12}+\frac{1}{4} \theta_{13}+\lambda \theta_{14}+\theta_{34}+\theta_{24}+\theta_{23}-\lambda \pi .
$$

Tomamos seu polinômio de Taylor truncado em ordem 7: $\operatorname{pol}_{7}(\lambda)=-44.6085+$ $555.822 \lambda-2958.75 \lambda^{2}+9132.45 \lambda^{3}-17329 \lambda^{4}+20103.8 \lambda^{5}-13163.6 \lambda^{6}+3754.07 \lambda^{7}$.

Os pontos de sela para essa função fase são:

$$
\begin{aligned}
& \lambda_{0}=0.320627+i 0.184734 \\
& \lambda_{1}=0.501289+i 0.247177 \\
& \lambda_{2}=0.680861+i 0.175808
\end{aligned}
$$

Fazendo $\lambda=x+i y$ para abrir $f(\lambda)=u_{7}(x, y)+y_{7}(x, y)$, obtemos

$$
\begin{gathered}
u_{7}(x, y)=-44.60853+555.8223 x-2958.75168 x^{2}+9132.44734 x^{3} \\
-17329.02036 x^{4}+20103.8275 x^{5}-13163.5797 x^{6}+3754.07097 x^{7}+2958.75168 y^{2} \\
-27397.34202 x y^{2}+103974.12218 x^{2} y^{2}-201038.2749 x^{3} y^{2}+197453.69555 x^{4} y^{2} \\
-78835.49033 x^{5} y^{2}-17329.02036 y^{4}+100519.13745 x y^{4}-197453.69554 x^{2} y^{4}
\end{gathered}
$$




$$
+131392.48388 x^{3} y^{4}+13163.5797 y^{6}-26278.49678 x y^{6} .
$$

e

$$
v_{7}(x, y)=555.82225 y-5917.50336 x y+27397.34202 x^{2} y-69316.08145 x^{3} y
$$$$
+100519.13744 x^{4} y-78981.47822 x^{5} y+26278.49678 x^{6} y-9132.44734 y^{3}+69316.08145 x y^{3}
$$$$
-201038.2749 x^{2} y^{3}+263271.59406 x^{3} y^{3}-131392.48388 x^{4} y^{3}+20103.82749 y^{5}
$$

$$
-78981.47822 x y^{5}+78835.49033 x^{2} y^{5}-3754.070967 y^{7} .
$$

Calculamos os $\gamma\left[x_{k}\right]=\gamma_{k}$ da fórmula do método.

$$
\begin{gathered}
\gamma_{0} \approx 0.167 \\
\gamma_{1} \approx-0.1 \\
\gamma_{2} \approx-0.0125
\end{gathered}
$$

Agora olhemos as contribuições de cada ponto:

- $\operatorname{pol}_{7}(\lambda)$ em $\lambda_{0}$ :

$$
\begin{gathered}
P_{4}(j) \approx e^{\frac{-i j 3 \pi}{8}} \frac{\sqrt{2 \pi}}{\sqrt{j} 1.88461}(2.31964-i 0.933097) e^{-i j \pi(-0.320627-i 0.184734)} e^{i j(3.3106+i 1.28643)} e^{i 1.67} \\
\approx \frac{1.33}{\sqrt{j}}(2.31964-i 0.933097) e^{j(-0.58036+i 1.01)} e^{i j(2.1325+i 1.28643)} e^{i 1.67} \\
\approx \frac{1.33}{\sqrt{j}} e^{i 1.67}(2.31964-i 0.933097) e^{j(-1.86679)} e^{i j(3.1425)}
\end{gathered}
$$

- $\operatorname{pol}_{7}(\lambda)$ em $\lambda_{1}$ :

$$
\begin{gathered}
P_{4}(j) \approx e^{\frac{-i j 3 \pi}{8}} \frac{\sqrt{2 \pi}}{\sqrt{j} 2.05229}(2.13618-i 0.0443922) e^{-i j \pi(-0.501289-i 0.247177)} e^{i j(4.03742+i 1.1997)} e^{-i 0.1} \\
\approx \frac{1.22}{\sqrt{j}}(2.13618-i 0.0443922) e^{j(-0.77653+i 1.57485)} e^{i j(2.85932+i 1.1997)} e^{-i 0.1} \\
\approx \frac{1.22}{\sqrt{j}} e^{-i 0.1}(2.13618-i 0.0443922) e^{j(-1.97623)} e^{i j(4.43417)}
\end{gathered}
$$

- $\operatorname{pol}_{7}(\lambda)$ em $\lambda_{2}$ :

$P_{4}(j) \approx e^{\frac{-i j 3 \pi}{8}} \frac{\sqrt{2 \pi}}{\sqrt{j}}(2.25699+i 0.928502) e^{-i j \pi(-0.680861-i 0.175808)} e^{i j(4.76223+i 1.16051)} e^{-i 0.0125}$ 


$$
\begin{gathered}
\approx \frac{1.13}{\sqrt{j}}(2.25699+i 0.928502) e^{j(-0.552317+i 2.138988)} e^{i j(3.58413+i 1.16051)} e^{-i 0.0125} \\
\approx \frac{1.13}{\sqrt{j}} e^{-i 0.0125}(2.25699+i 0.928502) e^{j(-1.712827)} e^{i j(5.72312)}
\end{gathered}
$$

Lembramos que estamos calculando as parcelas da integral inicial,

$$
Y_{j \lambda_{1} *_{1}}^{j \mu_{1}} Y_{j \lambda_{2}}^{j \mu_{2}} \approx \frac{(-1)^{j \frac{7}{4}+\frac{1}{2}}}{2 \pi^{3}} \sqrt{j}\left(P_{1}+P_{2}+P_{3}+P_{4}+P_{5}+P_{6}+P_{7}+P_{8}\right) .
$$

Calculamos até agora $P_{k}$ para $k=1,2,3,4$. Os restantes, lembremos que basta tomarmos o conjugado de cada um deses já calculados e assim obtemos:

$$
\begin{aligned}
& Y_{j \lambda_{1}}^{j \mu_{1}} *_{1} Y_{j \lambda_{2}}^{j \mu_{2}} \approx \frac{i(-1)^{j \frac{7}{4}}}{2 \pi^{3}} \sqrt{j} \sum_{k=1}^{8} P_{k} \\
& \approx \frac{i(-1)^{j \frac{7}{4}}}{2 \pi^{3}} \sqrt{j}\left(i \frac{1.044}{\sqrt{j}} e^{-i 1.03}(2.22622-i 1.0929) e^{j(-3.03202)} e^{i j 7.31713}\right. \\
& +i \frac{0.9}{\sqrt{j}} e^{i 24.2}(1.97798-i 0.060729) e^{j(-3.7993)} e^{i j(9.93288)} \\
& +i \frac{0.817}{\sqrt{j}} e^{i 16}(2.11392+i 1.09795) e^{j(-2.83845)} e^{i j(12.55789)} \\
& +i \frac{0.16956}{\sqrt{j}} e^{i 1.67}(2.27491-i 1.02783) e^{j(-0.83088)} e^{i j(6.88342)} \\
& +i \frac{0.196}{\sqrt{j}} e^{i 0.3}(2.04074-0.0546291 i) e^{j(-2.868583)} e^{i j(8.36201)} \\
& +i \frac{0.1726}{\sqrt{j}} e^{i 0.14}(2.18382+i 1.03107) e^{j(-2.2714)} e^{i j(9.12397)} \\
& +\frac{0.169564}{\sqrt{j}} e^{i 3.33}(2.27491-i 1.02783) e^{j(-2.44602)} e^{i j(4.06688)} \\
& +\frac{0.2776}{\sqrt{j}} e^{i 1.89}(2.04074-i 0.0546291) e^{j(-2.86858)} e^{i j(6.01)} \\
& +\frac{0.12204}{\sqrt{j}} e^{i 0.56}(2.18382+i 1.03107) e^{j(-2.2714)} e^{i j(7.94587)} \\
& +\frac{1.33}{\sqrt{j}} e^{i 1.67}(2.31964-i 0.933097) e^{j(-1.86679)} e^{i j(3.1425)} \\
& +\frac{1.22}{\sqrt{j}} e^{-i 0.1}(2.13618-i 0.0443922) e^{j(-1.97623)} e^{i j(4.43417)}
\end{aligned}
$$




$$
\begin{aligned}
& +\frac{1.13}{\sqrt{j}} e^{-i 0.0125}(2.25699+i 0.928502) e^{j(-1.712827)} e^{i j(5.72312)} \\
& -i \frac{1.044}{\sqrt{j}} e^{i 1.03}(2.22622+i 1.0929) e^{j(-3.03202)} e^{-i j 7.31713} \\
& -i \frac{0.9}{\sqrt{j}} e^{-i 24.2}(1.97798+i 0.060729) e^{j(-3.7993)} e^{-i j(9.93288)} \\
& -i \frac{0.817}{\sqrt{j}} e^{-i 16}(2.11392-i 1.09795) e^{j(-2.83845)} e^{-i j(12.55789)} \\
& -i \frac{0.16956}{\sqrt{j}} e^{-i 1.67}(2.27491+i 1.02783) e^{j(-0.83088)} e^{-i j(6.88342)} \\
& -i \frac{0.196}{\sqrt{j}} e^{-i 0.3}(2.04074+0.0546291 i) e^{j(-2.868583)} e^{-i j(8.36201)} \\
& -i \frac{0.1726}{\sqrt{j}} e^{-i 0.14}(2.18382-i 1.03107) e^{j(-2.2714)} e^{-i j(9.12397)} \\
& -\frac{0.169564}{\sqrt{j}} e^{-i 3.33}(2.27491+i 1.02783) e^{j(-2.44602)} e^{-i j(4.06688)} \\
& -\frac{0.2776}{\sqrt{j}} e^{-i 1.89}(2.04074+i 0.0546291) e^{j(-2.86858)} e^{-i j(6.01)} \\
& -\frac{0.12204}{\sqrt{j}} e^{-i 0.56}(2.18382-i 1.03107) e^{j(-2.2714)} e^{-i j(7.94587)} \\
& -\frac{1.33}{\sqrt{j}} e^{-i 1.67}(2.31964+i 0.933097) e^{j(-1.86679)} e^{-i j(3.1425)} \\
& -\frac{1.22}{\sqrt{j}} e^{i 0.1}(2.13618+i 0.0443922) e^{j(-1.97623)} e^{-i j(4.43417)} \\
& \left.-\frac{1.13}{\sqrt{j}} e^{i 0.0125}(2.25699-i 0.928502) e^{j(-1.712827)} e^{-i j(5.72312)}\right) \\
& \approx \frac{(-1)^{j \frac{7}{4}}}{2 \pi^{3}}\left(-1.044 e^{-i 1.03}(2.22622-i 1.0929) e^{j(-3.03202)} e^{i j 7.31713}\right. \\
& -0.9 e^{i 24.2}(1.97798-i 0.060729) e^{j(-3.7993)} e^{i j(9.93288)} \\
& -0.817 e^{i 16}(2.11392+i 1.09795) e^{j(-2.83845)} e^{i j(12.55789)} \\
& -0.16956 e^{i 1.67}(2.27491-i 1.02783) e^{j(-0.83088)} e^{i j(6.88342)} \\
& -0.196 e^{i 0.3}(2.04074-0.0546291 i) e^{j(-2.868583)} e^{i j(8.36201)} \\
& -0.1726 e^{i 0.14}(2.18382+i 1.03107) e^{j(-2.2714)} e^{i j(9.12397)} \\
& +i 0.169564 e^{i 3.33}(2.27491-i 1.02783) e^{j(-2.44602)} e^{i j(4.06688)}
\end{aligned}
$$




$$
\begin{aligned}
+ & i 0.2776 e^{i 1.89}(2.04074-i 0.0546291) e^{j(-2.86858)} e^{i j(6.01)} \\
+ & i 0.12204 e^{i 0.56}(2.18382+i 1.03107) e^{j(-2.2714)} e^{i j(7.94587)} \\
& +i 1.33 e^{i 1.67}(2.31964-i 0.933097) e^{j(-1.86679)} e^{i j(3.1425)} \\
+ & i 1.22 e^{-i 0.1}(2.13618-i 0.0443922) e^{j(-1.97623)} e^{i j(4.43417)} \\
+ & i 1.13 e^{-i 0.0125}(2.25699+i 0.928502) e^{j(-1.712827)} e^{i j(5.72312)} \\
& +1.044 e^{i 1.03}(2.22622+i 1.0929) e^{j(-3.03202)} e^{-i j 7.31713} \\
& +0.9 e^{-i 24.2}(1.97798+i 0.060729) e^{j(-3.7993)} e^{-i j(9.93288)} \\
+ & 0.817 e^{-i 16}(2.11392-i 1.09795) e^{j(-2.83845)} e^{-i j(12.55789)} \\
+ & 0.16956 e^{-i 1.67}(2.27491+i 1.02783) e^{j(-0.83088)} e^{-i j(6.88342)} \\
+ & 0.196 e^{-i 0.3}(2.04074+0.0546291 i) e^{j(-2.868583)} e^{-i j(8.36201)} \\
+ & 0.1726 e^{-i 0.14}(2.18382-i 1.03107) e^{j(-2.2714)} e^{-i j(9.12397)} \\
- & i 0.16957 e^{-i 3.33}(2.27491+i 1.02783) e^{j(-2.44602)} e^{-i j(4.06688)} \\
- & i 0.2776 e^{-i 1.89}(2.04074+i 0.0546291) e^{j(-2.86858)} e^{-i j(6.01)} \\
- & i 0.12204 e^{-i 0.56}(2.18382-i 1.03107) e^{j(-2.2714)} e^{-i j(7.94587)} \\
- & i 1.33 e^{-i 1.67}(2.31964+i 0.933097) e^{j(-1.86679)} e^{-i j(3.1425)} \\
- & i 1.22 e^{i 0.1}(2.13618+i 0.0443922) e^{j(-1.97623)} e^{-i j(4.43417)} \\
- & \left.i 1.13 e^{i 0.0125}(2.25699-i 0.928502) e^{j(-1.712827)} e^{-i j(5.72312)}\right)
\end{aligned}
$$

Vamos denotar em cada parcela por $a_{k}$ os termos em que não temos $j$, da seguinte maneira:

$$
\begin{gathered}
a_{1}=-1.044 e^{-i 1.03}(2.22622-i 1.0929), \quad a_{2}=-0.9 e^{i 24.2}(1.97798-i 0.060729), \\
a_{3}=-0.817 e^{i 16}(2.11392+i 1.09795), \quad a_{4}=-0.16956 e^{i 1.67}(2.27491-i 1.02783), \\
a_{5}=-0.196 e^{i 0.3}(2.04074-0.0546291 i), \quad a_{6}=-0.1726 e^{i 0.14}(2.18382+i 1.03107), \\
a_{7}=i 0.169564 e^{i 3.33}(2.27491-i 1.02783), \quad a_{8}=i 0.2776 e^{i 1.89}(2.04074-i 0.0546291) \\
a_{9}=i 0.12204 e^{i 0.56}(2.18382+i 1.03107), \quad a_{10}=i 1.33 e^{i 1.67}(2.31964-i 0.933097), \\
a_{11}=i 1.22 e^{-i 0.1}(2.13618-i 0.0443922), \quad a_{12}=i 1.13 e^{-i 0.0125}(2.25699+i 0.928502),
\end{gathered}
$$




$$
\begin{gathered}
a_{13}=1.044 e^{i 1.03}(2.22622+i 1.0929), \quad a_{14}=0.9 e^{-i 24.2}(1.97798+i 0.060729), \\
a_{15}=0.817 e^{-i 16}(2.11392-i 1.09795), \quad a_{16}=0.16956 e^{-i 1.67}(2.27491+i 1.02783), \\
a_{17}=0.196 e^{-i 0.3}(2.04074+i 0.0546291), \quad a_{18}=0.1726 e^{-i 0.14}(2.18382-i 1.03107), \\
a_{19}=-i 0.16957 e^{-i 3.33}(2.27491+i 1.02783), \quad a_{20}=-i 0.2776 e^{-i 1.89}(2.04074+i 0.0546291) \\
a_{21}=-i 0.12204 e^{-i 0.56}(2.18382-i 1.03107), q u a d a_{22}=-i 1.33 e^{-i 1.67}(2.31964+i 0.933097), \\
a_{23}=-i 1.22 e^{i 0.1}(2.13618+i 0.0443922), \quad a_{24}=-i 1.13 e^{i 0.0125}(2.25699-i 0.928502) .
\end{gathered}
$$

Ficando a soma:

$$
\begin{gathered}
\approx \frac{(-1)^{j \frac{7}{4}}}{2 \pi^{3}}\left(a_{1} e^{j(-3.03202)} e^{i j 7.31713}+a_{2} e^{j(-3.7993)} e^{i j(9.93288)}+a_{3} e^{j(-2.83845)} e^{i j(12.55789)}\right. \\
\quad+a_{4} e^{-j 0.83088} e^{i j 6.88342}+a_{5} e^{-j 2.868583} e^{i j 8.36201}+a_{6} e^{-j 2.2714} e^{i j 9.12397} \\
\quad+a_{7} e^{-j 2.44602} e^{i j 4.06688}+a_{8} e^{-j 2.86858} e^{i j 6.01}+a_{9} e^{-j 2.2714} e^{i j 7.94587} \\
\quad+a_{10} e^{-j 1.86679} e^{i j 3.1425}+a_{11} e^{-j 1.97623} e^{i j 4.43417}+a_{12} e^{-j 1.712827} e^{i j 5.72312} \\
+a_{13} e^{-j 3.03202} e^{-i j 7.31713}+a_{14} e^{-j 3.7993} e^{-i j 9.93288}+a_{15} e^{-j 2.83845} e^{-i j 12.55789} \\
+a_{16} e^{-j 0.83088} e^{-i j 6.88342}+a_{17} e^{-j 2.868583} e^{-i j 8.36201}+a_{18} e^{-j 2.2714} e^{-i j 9.12397} \\
+a_{19} e^{-j 2.44602} e^{-i j 4.06688}+a_{20} e^{-j 2.86858} e^{-i j 6.01}+a_{21} e^{-j 2.2714} e^{-i j 7.94587} \\
\left.+a_{22} e^{-j 1.86679} e^{-i j 3.1425}+a_{23} e^{-j 1.97623} e^{-i j 4.43417}+a_{24} e^{-j 1.712827} e^{-i j 5.72312}\right) .
\end{gathered}
$$

Vamos incorporar $\frac{1}{2 \pi^{3}}$ aos $a_{j}{ }^{\prime}$ s e fazer $(-1)^{j 7 / 4}=e^{i j 7 \pi / 4} \approx e^{i j 5.5}$ e distribuir para todas as parecelas, ficando então;

$$
\begin{aligned}
Y_{j \lambda_{1}}^{j \mu_{1}} *_{1} & Y_{j \lambda_{2}}^{j \mu_{2}} \approx\left(a_{1} e^{-j 3.03202} e^{i j 12.82}+a_{2} e^{-j 3.7993} e^{i j 15.43}+a_{3} e^{-j 2.83845} e^{i j 18.06}\right. \\
& +a_{4} e^{-j 0.83088} e^{i j 12.38}+a_{5} e^{-j 2.868583} e^{i j 13.86}+a_{6} e^{-j 2.2714} e^{i j 14.62} \\
& +a_{7} e^{-j 2.44602} e^{i j 9.57}+a_{8} e^{-j 2.86858} e^{i j 11.51}+a_{9} e^{-j 2.2714} e^{i j 13.44} \\
& +a_{10} e^{-j 1.86679} e^{i j 8.64}+a_{11} e^{-j 1.97623} e^{i j 9.93}+a_{12} e^{-j 1.712827} e^{i j 11.22} \\
+ & a_{13} e^{-j 3.03202} e^{-i j 1.82}+a_{14} e^{-j 3.7993} e^{-i j 4.43}+a_{15} e^{-j 2.83845} e^{-i j 7.06} \\
+ & a_{16} e^{-j 0.83088} e^{-i j 1.38}+a_{17} e^{-j 2.868583} e^{-i j 2.86}+a_{18} e^{-j 2.2714} e^{-i j 3.62} \\
+ & a_{19} e^{-j 2.44602} e^{i j 1.43}+a_{20} e^{-j 2.86858} e^{-i j 0.51}+a_{21} e^{-j 2.2714} e^{-i j 2.46}
\end{aligned}
$$




$$
\left.+a_{22} e^{-j 1.86679} e^{i j 2.36}+a_{23} e^{-j 1.97623} e^{i j 1.07}+a_{24} e^{-j 1.712827} e^{-i j 0.22}\right)
$$

Observamos que as 24 parcelas irão para zero quando $j \rightarrow \infty$, pois $\left|e^{i j \omega}\right|$ é limitada e $e^{-j \xi}$ vai a zero quando $j$ tende a infinto. E assim conluímos que:

$$
Y_{j \lambda_{1}}^{j \mu_{1}} *_{1} Y_{j \lambda_{2}}^{j \mu_{2}} \rightarrow 0
$$

Analisemos com que "velocidade"esse produto irá a zero, tomando o módulo e observamos:

$Y_{j \lambda_{1}}^{j \mu_{1}} *_{1} Y_{j \lambda_{2}}^{j \mu_{2}} \leq\left|a_{1}\right| e^{-j 3.03202}+\left|a_{2}\right| e^{-j 3.7993}+\left|a_{3}\right| e^{-j 2.83845}+\left|a_{4}\right| e^{-j 0.83088}+\left|a_{5}\right| e^{-j 2.868583}+\left|a_{6}\right| e^{-j 2.2714}$

$+\left|a_{7}\right| e^{-j 2.44602}+\left|a_{8}\right| e^{-j 2.86858}+\left|a_{9}\right| e^{-j 2.2714}+\left|a_{10}\right| e^{-j 1.86679}+\left|a_{11}\right| e^{-j 1.97623}+\left|a_{12}\right| e^{-j 1.712827}$

$+\left|a_{13}\right| e^{-j 3.03202}+a_{14} e^{-j 3.7993}+\left|a_{15}\right| e^{-j 2.83845}+\left|a_{16}\right| e^{-j 0.83088}+\left|a_{17}\right| e^{-j 2.868583}+\left|a_{18}\right| e^{-j 2.2714}$

$+\left|a_{19}\right| e^{-j 2.44602}+\left|a_{20}\right| e^{-j 2.86858}+\left|a_{21}\right| e^{-j 2.2714}+\left|a_{22}\right| e^{-j 1.86679}+\left|a_{23}\right| e^{-j 1.97623}+\left|a_{24}\right| e^{-j 1.712827}$

Observamos que a parcela de maior potência é a que dominará todas as outras, ou seja,

$$
Y_{j \lambda_{1} *_{1}}^{j \mu_{1}} Y_{j \lambda_{2}}^{j \mu_{2}} \leq N_{0} e^{-0.83088 j}
$$

$\operatorname{com} N_{0} \in \mathbb{R}$.

\subsection{Cálculo da aproximação para o produto de Berezin}

Calculemos agora, ainda no exemplo, o produto de Berezin para $\lambda_{1}=1 / 2$ e $\lambda_{2}=$ $1 / 4$

$$
Y_{j \lambda_{1}}^{j \mu_{1}}{ }_{b} Y_{j \lambda_{2}}^{j \mu_{2}} \sim \frac{\widetilde{g}_{2} g_{3}}{8} \sqrt{j} \int_{1 / 4}^{3 / 4} \frac{g_{1}}{\left(4-\lambda^{2}\right)^{1 / 4}\left[(2+\lambda)^{2+\lambda}(2-\lambda)^{2-\lambda}\right]^{j / 2}} e^{i j f(\lambda)} d \lambda,
$$

onde,

$$
\frac{\widetilde{g}_{2} g_{3}}{8} \approx i \frac{e^{i j 11 \pi / 4} 0.34685}{\pi^{2}} \frac{17.3^{j}}{2^{2 j}}
$$

Inciemos com a aproximação para $Q_{1}$ :

$$
Q_{1}(j)=i e^{i j 3 \pi / 8} \int_{1 / 4}^{3 / 4} \frac{g_{1}}{\left(4-\lambda^{2}\right)^{1 / 4}\left[(2+\lambda)^{2+\lambda}(2-\lambda)^{2-\lambda}\right] j / 2} e^{i j f(\lambda)} d \lambda
$$


onde a $f(\lambda)$ é a função fase,

$$
f(\lambda)=\frac{1}{2} \theta_{12}+\frac{1}{4} \theta_{13}+\lambda \theta_{14}+\theta_{34}+\theta_{24}+\theta_{23}-\lambda \pi .
$$

Tomamos seu polinômio de Taylor truncado em ordem 7:

$$
\begin{gathered}
\operatorname{pol}_{7}(\lambda)=-44.6085+562.105 \lambda-2958.75 \lambda^{2} \\
+9132.45 \lambda^{3}-17329 \lambda^{4}+20103.8 \lambda^{5}-13163.6 \lambda^{6}+3754.07 \lambda^{7}
\end{gathered}
$$

Os pontos de sela para essa função fase são:

$$
\begin{aligned}
& \lambda_{0}=0.291197+i 0.185923 \\
& \lambda_{1}=0.500299+i 0.287617 \\
& \lambda_{2}=0.711282+i 0.179422
\end{aligned}
$$

Fazendo $\lambda=x+i y$ para abrir $f(\lambda)=u_{7}(x, y)+y_{7}(x, y)$, obtemos

$$
\begin{gathered}
u_{7}(x, y)=-44.6085+562.105 x-2958.75 x^{2}+9132.45 x^{3}-17329 x^{4}+20103.8 x^{5} \\
-13163.6 x^{6}+ \\
+3754.07 x^{7}+2958.75 y^{2}-27397.3 x y^{2}+103974 x^{2} y^{2}-201038 x^{3} y^{2} \\
+197454 x^{4} y^{2}-78835.5 x^{5} y^{2}-17329 . y^{4}+100519 . x y^{4} \\
-197454 x^{2} y^{4}+131392 x^{3} y^{4}+13163.6 y^{6}-26278.5 x y^{6}
\end{gathered}
$$

e

$$
\begin{gathered}
v_{7}(x, y)=558.964 y-5917.5 x y+27397.3 x^{2} y-69316.1 x^{3} y+100519 x^{4} y \\
-78981.5 x^{5} y+26278.5 x^{6} y-9132.45 y^{3}+69316.1 x y^{3}-201038 . x^{2} y^{3}+263272 . x^{3} y^{3} \\
-131392 . x^{4} y^{3}+20103.8 y^{5}-78981.5 x y^{5}+78835.5 x^{2} y^{5}-3754.07 y^{7}
\end{gathered}
$$

Calculamos os $\gamma\left[x_{k}\right]=\gamma_{k}$ da fórmula do método.

$$
\begin{gathered}
\gamma_{0} \approx 1.03 \\
\gamma_{1} \approx 24.2 \\
\gamma_{2} \approx 16 .
\end{gathered}
$$

Agora olhemos as contribuições de cada ponto: 
- $\operatorname{pol}_{7}(\lambda)$ em $\lambda_{0}$ :

$$
\begin{gathered}
Q_{1}(j) \approx i e^{\frac{i j 3 \pi}{8}} \frac{\sqrt{2 \pi}}{\sqrt{j} 2.40194}(1.58428-i 0.764329) e^{-i j \pi(-0.291197-i 0.185923)} \\
(16.3795+i 0.89136)^{-j / 2} e^{i j(5.22423+i 2.44792)} e^{i 1.03} \\
\approx i \frac{1.044}{\sqrt{j}} e^{i 1.03}(1.58428-i 0.764329) e^{i j 7.3171} e^{-j 3.032}(16.3795+i 0.89136)^{-j / 2}
\end{gathered}
$$

- $\operatorname{pol}_{7}(\lambda)$ em $\lambda_{1}$ :

$$
\begin{gathered}
Q_{1}(j) \approx i e^{\frac{i j 3 \pi}{8}} \frac{\sqrt{2 \pi}}{\sqrt{j} 2.78294}(1.41326-i 0.0168961) e^{-i j \pi(-0.500299-i 0.287617)} \\
(17.1925+i 2.52637)^{-j / 2} e^{i j(7.18304+i 2.89576)} e^{i 24.2} \\
\approx i \frac{0.901}{\sqrt{j}} e^{i 24.2}(1.41326-i 0.0168961) e^{i j 9.27594} e^{-j 3.79936}(17.1925+i 2.52637)^{-j / 2}
\end{gathered}
$$

- $\operatorname{pol}_{7}(\lambda)$ em $\lambda_{2}$ :

$$
\begin{gathered}
Q_{1}(j) \approx i e^{\frac{i j 3 \pi}{8}} \frac{\sqrt{2 \pi}}{\sqrt{j} 3.06983}(1.5269+i 0.828414) \\
e^{-i j \pi(-0.711282-i 0.179422)}(20.1644+i 2.69787)^{-j / 2} e^{i j(9.14521+i 2.27478)} e^{i 16} \\
\approx i \frac{0.81654}{\sqrt{j}}(1.5269+i 0.828414) e^{j(-0.56367+i 2.23456)}(20.1644+i 2.69787)^{-j / 2} e^{i j(10.32331+i 2.27478)} e^{i 16} \\
\approx i \frac{0.81654}{\sqrt{j}} e^{i 16}(1.5269+i 0.828414)(20.1644+i 2.69787)^{-j / 2} e^{-j 2.83845} e^{i j 12.5579}
\end{gathered}
$$

Próxima parcela $Q_{2}$, e repitiremos a "receita"usada para encontrar $Q_{1}$ :

$$
Q_{2}(j)=i e^{i j 3 \pi / 8} \int_{1 / 4}^{3 / 4} \frac{g_{1}}{\left(4-\lambda^{2}\right)^{1 / 4}\left[(2+\lambda)^{2+\lambda}(2-\lambda)^{2-\lambda}\right]^{j / 2}} e^{i j f(\lambda)} d \lambda,
$$

onde a $f(\lambda)$ é a função fase,

$$
f(\lambda)=\frac{1}{2} \theta_{12}+\frac{1}{4} \theta_{13}+\lambda \theta_{14}+\theta_{34}+\theta_{24}+\theta_{23},
$$

Encontramos o mesmo ponto de inflexão no mesmo intervalo. Porém a $f_{3}(\lambda)$ agora tem uma sutil diferença, não temos a parcela $\lambda \pi$. Assim,

$$
f_{3}(\lambda)=\frac{1}{2}\left(\pi-\theta_{12}\right)+\frac{1}{4} \theta_{13}+\lambda \theta_{14}+\pi-\theta_{34}+\theta_{24}+\theta_{23} .
$$


Tomamos seu polinômio de Taylor truncado em ordem 7:

- $\operatorname{pol}_{7}(\lambda)$ :

$$
\begin{gathered}
\operatorname{pol}_{7}(\lambda)=-44.6085+558.964 \lambda-2958.75 \lambda^{2}+9132.45 \lambda^{3}-17329 . \lambda^{4} \\
+20103.8 \lambda^{5}-13163.6 \lambda^{6}+3754.07 \lambda^{7}
\end{gathered}
$$

com os seguintes pontos de sela que nos interessam (lembrando que foram escolhidos justamente os que mantem a parte imaginária positiva);

$$
\begin{gathered}
\lambda_{0}=0.303903+i 0.18464, \\
\lambda_{1}=0.500573+i 0.27115, \\
\lambda_{2}=0.698302+i 0.177222 .
\end{gathered}
$$

Escrevendo $f(\lambda) \approx \operatorname{pol}_{7}(\lambda)=u_{7}(x, y)+i v_{7}(x, y)$, fazendo $\lambda=x+i y$, com

$$
\begin{gathered}
u_{7}(x, y)=-44.60853+558.96385 x-2958.75168 x^{2}+9132.44734 x^{3}-17329.02036 x^{4} \\
+20103.827489 x^{5}-13163.5797 x^{6}+3754.07097 x^{7}+2958.75168 y^{2}-27397.34202 x y^{2} \\
+103974.12218 x^{2} y^{2}-201038.2749 x^{3} y^{2}+197453.69555 x^{4} y^{2}-78835.49033 x^{5} y^{2} \\
-17329.020363 y^{4}+100519.13745 x y^{4}-197453.69555 x^{2} y^{4}+131392.48388 x^{3} y^{4} \\
+13163.5797 y^{6}-26278.49678 x y^{6} \\
v_{7}(x, y)=558.96385 y-5917.50336 x y+27397.34202 x^{2} y-69316.08145 x^{3} y \\
+100519.13745 x^{4} y-78981.47822 x^{5} y+26278.49678 x^{6} y-9132.44734 y^{3}+69316.08145 x y^{3} \\
-201038.2749 x^{2} y^{3}+263271.5941 x^{3} y^{3}-131392.48388 x^{4} y^{3}+20103.82749 y^{5} \\
-78981.47822 x y^{5}+78835.49033 x^{2} y^{5}-3754.07097 y^{7}
\end{gathered}
$$

Os valores de $\gamma\left[x_{k}\right]=\gamma_{k}$, para $k=0,1,2$, da fórmula usada no método de "steepest descents", para essa parcela são dados por:

$$
\gamma_{0} \approx 0.167, \pi
$$




$$
\begin{gathered}
\gamma_{1} \approx 0.3 \\
\gamma_{2} \approx 0.14 .
\end{gathered}
$$

Fazendo os mesmos cálculos, obtemos a seguinte aproximação para essa integral, usando as contribuições dadas pelo polinômio de Taylor truncado de ordem 7 e usando 3.3.1:

- $\operatorname{pol}_{7}(\lambda)$ em $\lambda_{0}$ :

$$
\begin{gathered}
Q_{2}(j) \approx i e^{i j \frac{3 \pi}{8}} \frac{\sqrt{2 \pi}}{\sqrt{j} 2.16298} e^{i j(4.29024+i 1.86602)}(1.6195-i 0.717875) \\
e^{-i j \pi(-0.303903-i 0.18464)}(16.4436+i 0.928146)^{-j / 2} e^{i 1.67} \\
\approx i \frac{1.15887}{\sqrt{j}} e^{i j(5.46834+i 1.86602)}(1.6195-i 0.717875) e^{j(-0.58+i 0.954739)}(16.4436+i 0.928146)^{-j / 2} e^{i 1.67} \\
\approx i \frac{1.15887}{\sqrt{j}} e^{i 1.67}(1.6195-i 0.717875) e^{i j 6.423079} e^{-j 2.44602}(16.4436+i 0.928146)^{-j / 2} \\
\bullet p o l_{7}(\lambda) \mathrm{em} \lambda_{1}: \\
Q_{2}(j) \approx i e^{i j \frac{3 \pi}{8}} \frac{\sqrt{2 \pi}}{\sqrt{j} 12.7689}(1.45899+i 0.0131905) \\
\approx i \frac{0.196}{\sqrt{j}}(1.45899+i 0.0131905) e^{j(0.85184+i 1.5726)}(17.2989-i 2.39797)^{-j / 2} e^{i j(6.78904+i 2.01674)} e^{i 0.3} \\
\approx i \frac{0.196}{\sqrt{j}} e^{i 0.3}(1.45899+i 0.0131905)(17.2989-i 2.39797)^{-j / 2} e^{-j 1.1649} e^{i j 8.36164}
\end{gathered}
$$

- $\operatorname{pol}_{7}(\lambda)$ em $\lambda_{2}$ :

$$
\begin{gathered}
Q_{2}(j) \approx i e^{i j \frac{3 \pi}{8}} \frac{\sqrt{2 \pi}}{\sqrt{j} 14.5245}(1.57736+i 0.778645) \\
e^{-i j \pi(-0.698302-i 0.177222)}(19.9945+i 2.589)^{-j / 2} e^{i j(6.93019+i 1.71464)} e^{i 0.14} \\
\approx i \frac{0.172579}{\sqrt{j}}(1.57736+i 0.778645) e^{j(-0.55676-i 0.177222)}(19.9945+i 2.589)^{-j / 2} e^{i j(8.10829+i 1.71464)} e^{i 0.14} \\
\approx i e^{i j \frac{3 \pi}{8}} \frac{0.172579}{\sqrt{j}} e^{i 0.14}(1.57736+i 0.778645)(19.9945+i 2.589)^{-j / 2} e^{-j 2.2714} e^{i j 10.30207}
\end{gathered}
$$


Calculando para $Q_{3}$ :

$$
Q_{3}(j)=e^{-3 \pi / 8} \int_{1 / 4}^{3 / 4} \frac{g_{1}}{\left(4-\lambda^{2}\right)^{1 / 4}\left[(2+\lambda)^{2+\lambda}(2-\lambda)^{2-\lambda}\right]^{j / 2}} e^{i j f(\lambda)} d \lambda,
$$

onde a $f(\lambda)$ é a função fase,

$$
f(\lambda)=\frac{1}{2} \theta_{12}+\frac{1}{4} \theta_{13}+\lambda \theta_{14}+\theta_{34}+\theta_{24}+\theta_{23}
$$

Tomamos seu polinômio de Taylor, truncado em ordem 7:

$\operatorname{pol}_{7}(\lambda)=-44.6085+558.964 \lambda-2958.75 \lambda^{2}+9132.45 \lambda^{3}-17329 \lambda^{4}+20103.8 \lambda^{5}-13163.6 \lambda^{6}+3754.07 \lambda^{7}$, com os seguinte pontos de sela que nos interessam:

$$
\begin{aligned}
& \lambda_{0}=0.303903+i 0.18464, \\
& \lambda_{1}=0.500573+i 0.27115, \\
& \lambda_{2}=0.698302+i 0.177222 .
\end{aligned}
$$

Observamos que a função fase é a mesma da $Q_{2}$ a menos de uma constante $\frac{-j 3 \pi}{8}$.

Escrevendo $f(\lambda) \approx \operatorname{pol}_{7}(\lambda)=u_{7}(x, y)+i v_{7}(x, y)$, fazendo $\lambda=x+i y$, onde

$$
\begin{gathered}
u_{7}(x, y)=-44.60853+558.96384 x-2958.75168 x^{2}+9132.44734 x^{3}-17329.02036 x^{4} \\
+20103.82749 x^{5}-13163.5797 x^{6}+3754.07097 x^{7}+2958.75168 y^{2} \\
-27397.34202 x y^{2}+103974.1222 x^{2} y^{2}-201038.2749 x^{3} y^{2}+197453.695545 x^{4} y^{2} \\
-78835.49033 x^{5} y^{2}-17329.020363 y^{4}+100519.13745 x y^{4}-197453.69555 x^{2} y^{4} \\
+131392.48388 x^{3} y^{4}+13163.5797 y^{6}-26278.496775 x y^{6}
\end{gathered}
$$

e

$$
\begin{gathered}
v_{7}(x, y)=558.96385 y-5917.50336 x y+27397.34202 x^{2} y-69316.08145 x^{3} y \\
+100519.13745 x^{4} y-78981.47822 x^{5} y+26278.49678 x^{6} y-9132.44734 y^{3} \\
+69316.08145 x y^{3}-201038.2749 x^{2} y^{3}+263271.59406 x^{3} y^{3}-131392.48388 x^{4} y^{3} \\
+20103.82749 y^{5}-78981.47822 x y^{5}+78835.490323 x^{2} y^{5}-3754.070968 y^{7} .
\end{gathered}
$$

Calculando os $\gamma\left[x_{k}\right]=\gamma_{k}$ da fórmula do método: 


$$
\begin{aligned}
& \gamma_{0} \approx 3.33, \\
& \gamma_{1} \approx 1.89, \\
& \gamma_{2} \approx 0.56 .
\end{aligned}
$$

E as contribuições para cada caso:

- $\operatorname{pol}_{7}(\lambda)$ em $\lambda_{0}$ :

$$
\begin{aligned}
& Q_{3}(j) \approx e^{\frac{-i j 3 \pi}{8}} \frac{\sqrt{2 \pi}}{\sqrt{j} 14.7828}(1.6195-i 0.717875) \\
& e^{-i j \pi(-0.303903-i 0.18464)}(16.4436+i 0.928146)^{-j / 2} e^{i j(4.29024+i 1.86602)} e^{i 3.33} \\
& \approx \frac{0.169564}{\sqrt{j}}(1.6195-i 0.717875) e^{j(-0.58+i 0.954739)}(16.4436+i 0.928146)^{-j / 2} e^{i j(3.11214+i 1.86602)} e^{i 3.33} \\
& \approx \frac{0.169564}{\sqrt{j}} e^{i 3.33}(1.6195-i 0.717875)(16.4436+i 0.928146)^{-j / 2} e^{-j 2.44602} e^{i j 4.066879} \\
& \text { - } \operatorname{pol}_{7}(\lambda) \text { em } \lambda_{1} \text { : } \\
& \begin{array}{c}
Q_{3}(j) \approx e^{\frac{-i j 3 \pi}{8}} \frac{\sqrt{2 \pi}}{\sqrt{j} 12.7689}(1.45899-i 0.0131905) \\
e^{-i j \pi(-0.500573-i 0.27115)}(17.2989+i 2.39797)^{-j / 2} e^{i j(5.61094+i 2.01674)} e^{i 1.89} \\
\approx \frac{0.196307}{\sqrt{j}}(1.45899-i 0.0131905) e^{j(-0.851937+i 1.572586)}(17.2989+i 2.39797)^{-j / 2} e^{i j(6.78904+i 2.01674)} e^{i 1.89} \\
\approx \frac{0.196307}{\sqrt{j}} e^{i 1.89}(1.45899-i 0.0131905)(17.2989+i 2.39797)^{-j / 2} e^{-j 2.868677} e^{i j 8.361626} \\
\bullet \operatorname{pol}_{7}(\lambda) \mathrm{em} \lambda_{2}:
\end{array} \\
& Q_{3}(j) \approx e^{\frac{-i j 3 \pi}{8}} \frac{\sqrt{2 \pi}}{\sqrt{j} 14.5245}(1.57736+i 0.778645) \\
& e^{-i j \pi(-0.698302-i 0.177222)}(19.9945+i 2.589)^{-j / 2} e^{i j(6.93019+i 1.71464)} e^{i 0.56} \\
& \approx \frac{0.12204}{\sqrt{j}}(1.57736+i 0.778645) e^{j(-0.556759+i 2.19378)}(19.9945+i 2.589)^{-j / 2} e^{i j(6.93019+i 1.71464)} e^{i 0.56} \\
& \approx \frac{0.12204}{\sqrt{j}} e^{i 0.56}(1.57736+i 0.778645)(19.9945+i 2.589)^{-j / 2} e^{-j 2.2714} e^{i j 9.12397}
\end{aligned}
$$

E por ultimo calculamos a aproximação para $Q_{4}$ : 


$$
Q_{4}(j)=e^{\frac{-i j 3 \pi}{8}} \int_{1 / 4}^{3 / 4} \frac{g_{1}}{\left(4-\lambda^{2}\right)^{1 / 4}\left[(2+\lambda)^{2+\lambda}(2-\lambda)^{2-\lambda}\right]^{j / 2}} e^{i j f(\lambda)} d \lambda,
$$

onde a $f(\lambda)$ é a função fase,

$$
f(\lambda)=\frac{1}{2} \theta_{12}+\frac{1}{4} \theta_{13}+\lambda \theta_{14}+\theta_{34}+\theta_{24}+\theta_{23}-\lambda \pi .
$$

Tomamos seu polinômio de Taylor truncado em ordem 7: $\operatorname{pol}_{7}(\lambda)=-44.6085+$ $555.822 \lambda-2958.75 \lambda^{2}+9132.45 \lambda^{3}-17329 \lambda^{4}+20103.8 \lambda^{5}-13163.6 \lambda^{6}+3754.07 \lambda^{7}$.

Os pontos de sela para essa função fase são:

$$
\begin{aligned}
& \lambda_{0}=0.320627+i 0.184734 \\
& \lambda_{1}=0.501289+i 0.247177 \\
& \lambda_{2}=0.680861+i 0.175808
\end{aligned}
$$

Fazendo $\lambda=x+i y$ para abrir $f(\lambda)=u_{7}(x, y)+y_{7}(x, y)$, obtemos

$$
u_{7}(x, y)=-44.60853+555.8223 x-2958.75168 x^{2}+9132.44734 x^{3}
$$

$-17329.02036 x^{4}+20103.8275 x^{5}-13163.5797 x^{6}+3754.07097 x^{7}+2958.75168 y^{2}$

$-27397.34202 x y^{2}+103974.12218 x^{2} y^{2}-201038.2749 x^{3} y^{2}+197453.69555 x^{4} y^{2}$

$-78835.49033 x^{5} y^{2}-17329.02036 y^{4}+100519.13745 x y^{4}-197453.69554 x^{2} y^{4}$

$$
+131392.48388 x^{3} y^{4}+13163.5797 y^{6}-26278.49678 x y^{6} .
$$

e

$$
v_{7}(x, y)=555.82225 y-5917.50336 x y+27397.34202 x^{2} y-69316.08145 x^{3} y
$$

$+100519.13744 x^{4} y-78981.47822 x^{5} y+26278.49678 x^{6} y-9132.44734 y^{3}+69316.08145 x y^{3}$

$-201038.2749 x^{2} y^{3}+263271.59406 x^{3} y^{3}-131392.48388 x^{4} y^{3}+20103.82749 y^{5}$

$$
-78981.47822 x y^{5}+78835.49033 x^{2} y^{5}-3754.070967 y^{7} .
$$

Calculamos os $\gamma\left[x_{k}\right]$ da fórmula do método.

$$
\begin{aligned}
& \gamma[0.303903] \approx 0.167 \\
& \gamma[0.500573] \approx-0.1
\end{aligned}
$$




$$
\gamma[0.69302] \approx-0.0125
$$

Agora olhemos as contribuições de cada ponto:

- $\operatorname{pol}_{7}(\lambda)$ em $\lambda_{0}$ :

$$
\begin{gathered}
Q_{4}(j) \approx e^{\frac{-i j 3 \pi}{8}} \frac{\sqrt{2 \pi}}{\sqrt{j} 12.6322}(1.65211-i 0.650164) \\
e^{-i j \pi(-0.320627-i 0.184734)}(16.5263+i 0.985628)^{-j / 2} e^{i j(3.3106+i 1.28643)} e^{i 1.67} \\
\approx \frac{0.1984}{\sqrt{j}}(1.65211-i 0.650164) e^{j(-0.5804+i 1.007)}(16.5263+i 0.985628)^{-j / 2} e^{i j(2.1325+i 1.28643)} e^{i 1.67} \\
\approx \frac{0.1984}{\sqrt{j}} e^{i 1.67}(1.65211-i 0.650164)(16.5263+i 0.985628)^{-j / 2} e^{-j 1.86683} e^{i j(3.1395)}
\end{gathered}
$$

- $\operatorname{pol}_{7}(\lambda)$ em $\lambda_{1}$ :

$$
\begin{gathered}
Q_{4}(j) \approx e^{\frac{-i j 3 \pi}{8}} \frac{\sqrt{2 \pi}}{\sqrt{j} 10.1603}(1.52852-i 0.00693866) \\
\quad e^{-i j \pi(-0.501289-i 0.247177)}(17.4468+i 2.20811)^{-j / 2} e^{i j(4.03742+i 1.1997)} e^{-i 0.1} \\
\approx \frac{0.24671}{\sqrt{j}}(1.52852-i 0.00693866) e^{j(-0.77653+i 1.57485)}(17.4468+i 2.20811)^{-j / 2} e^{i j(2.85932+i 1.1997)} e^{-i 0.1} \\
\approx \frac{0.24671}{\sqrt{j}} e^{-i 0.1}(1.52852-i 0.00693866)(17.4468+i 2.20811)^{-j / 2} e^{-j 1.97623} e^{i j 4.43417} \\
\bullet p_{7}(\lambda) \mathrm{em} \lambda_{2}: \\
\quad Q_{4}(j) \approx e^{\frac{-i j 3 \pi}{8}} \frac{\sqrt{2 \pi}}{\sqrt{j} 12.2753}(1.6298+i 0.702632) \\
\approx \frac{0.2042}{\sqrt{j}}(1.6298+i 0.702632) e^{j(-0.5523+i 2.138988)}(19.7645+i 2.46914)^{-j / 2} e^{i j(3.58413+i 1.16051)} e^{-i 0.0125} \\
\approx \frac{0.2042}{\sqrt{j}} e^{-i 0.0125}(1.6298+i 0.702632)(19.7645+i 2.46914)^{-j / 2} e^{-j 1.71281} e^{i j 5.72312}
\end{gathered}
$$

Voltando à integral de Berezin e somando todas as contribuições temos:

$$
Y_{j \lambda_{1}}^{j \mu_{1}} \star \vec{b} Y_{j \lambda_{2}}^{j \mu_{2}} \approx
$$

$i 0.34685 \frac{e^{\frac{i j 11 \pi}{4}}}{\pi^{2}} \frac{(17.3)^{j}}{2^{2 j}} \sqrt{j}\left(i \frac{1.044}{\sqrt{j}} e^{i 1.03}(1.58428-i 0.764329) e^{i j 7.3171} e^{-j 3.032}(16.3795+i 0.89136)^{-j / 2}\right.$ 


$$
\begin{aligned}
& +i \frac{0.901}{\sqrt{j}} e^{i 24.2}(1.41326-i 0.0168961) e^{i j 9.27594} e^{-j 3.79936}(17.1925+i 2.52637)^{-j / 2} \\
& i \frac{0.81654}{\sqrt{j}} e^{i 16}(1.5269+i 0.828414)(20.1644+i 2.69787)^{-j / 2} e^{-j 2.83845} e^{i j 12.5579} \\
& +i \frac{1.15887}{\sqrt{j}} e^{i 1.67}(1.6195-i 0.717875) e^{i j 6.423079} e^{-j 2.44602}(16.4436+i 0.928146)^{-j / 2} \\
& +i \frac{0.196}{\sqrt{j}} e^{i 0.3}(1.45899+i 0.0131905)(17.2989-i 2.39797)^{-j / 2} e^{-j 1.1649} e^{i j 8.36164} \\
& +i e^{i j \frac{3 \pi}{8}} \frac{0.172579}{\sqrt{j}} e^{i 0.14}(1.57736+i 0.778645)(19.9945+i 2.589)^{-j / 2} e^{-j 2.2714} e^{i j 10.30207} \\
& +\frac{0.169564}{\sqrt{j}} e^{i 3.33}(1.6195-i 0.717875)(16.4436+i 0.928146)^{-j / 2} e^{-j 2.44602} e^{i j 4.066879} \\
& +\frac{0.196307}{\sqrt{j}} e^{i 1.89}(1.45899-i 0.0131905)(17.2989+i 2.39797)^{-j / 2} e^{-j 2.868677} e^{i j 8.361626} \\
& +\frac{0.12204}{\sqrt{j}} e^{i 0.56}(1.57736+i 0.778645)(19.9945+i 2.589)^{-j / 2} e^{-j 2.2714} e^{i j 9.12397} \\
& +\frac{0.1984}{\sqrt{j}} e^{i 1.67}(1.65211-i 0.650164)(16.5263+i 0.985628)^{-j / 2} e^{-j 1.86683} e^{i j(3.1395)} \\
& +\frac{0.24671}{\sqrt{j}} e^{-i 0.1}(1.52852-i 0.00693866)(17.4468+i 2.20811)^{-j / 2} e^{-j 1.97623} e^{i j 4.43417} \\
& +\frac{0.2042}{\sqrt{j}} e^{-i 0.0125}(1.6298+i 0.702632)(19.7645+i 2.46914)^{-j / 2} e^{-j 1.71281} e^{i j 5.72312} \\
& +\frac{0.1984}{\sqrt{j}} e^{-i 1.67}(1.65211+i 0.650164)(16.5263-i 0.985628)^{-j / 2} e^{-j 1.86683} e^{-i j(3.1395)} \\
& +\frac{0.24671}{\sqrt{j}} e^{i 0.1}(1.52852+i 0.00693866)(17.4468-i 2.20811)^{-j / 2} e^{-j 1.97623} e^{-i j 4.43417} \\
& +\frac{0.2042}{\sqrt{j}} e^{i 0.0125}(1.6298-i 0.702632)(19.7645-i 2.46914)^{-j / 2} e^{-j 1.71281} e^{-i j 5.72312} \\
& +\frac{0.169564}{\sqrt{j}} e^{-i 3.33}(1.6195+i 0.717875)(16.4436-i 0.928146)^{-j / 2} e^{-j 2.44602} e^{-i j 4.066879} \\
& +\frac{0.196307}{\sqrt{j}} e^{-i 1.89}(1.45899+i 0.0131905)(17.2989-i 2.39797)^{-j / 2} e^{-j 2.868677} e^{-i j 8.361626} \\
& +\frac{0.12204}{\sqrt{j}} e^{-i 0.56}(1.57736-i 0.778645)(19.9945-i 2.589)^{-j / 2} e^{-j 2.2714} e^{-i j 9.12397} \\
& -i \frac{1.15887}{\sqrt{j}} e^{-i 1.67}(1.6195+i 0.717875)(16.4436-i 0.928146)^{-j / 2} e^{-i j 6.423079} e^{-j 2.44602}
\end{aligned}
$$




$$
\begin{aligned}
& -i \frac{0.196}{\sqrt{j}} e^{-i 0.3}(1.45899-i 0.0131905)(17.2989+i 2.39797)^{-j / 2} e^{-j 1.1649} e^{-i j 8.36164} \\
& -i \frac{0.172579}{\sqrt{j}} e^{-i 0.14}(1.57736-i 0.778645)(19.9945-i 2.589)^{-j / 2} e^{-j 2.2714} e^{-i j 10.30207} \\
& -i \frac{1.044}{\sqrt{j}} e^{-i 1.03}(1.58428+i 0.764329)(16.3795-i 0.89136)^{-j / 2} e^{-i j 7.3171} e^{-j 3.032} \\
& -i \frac{0.901}{\sqrt{j}} e^{-i 24.2}(1.41326+i 0.0168961)(17.1925-i 2.52637)^{-j / 2} e^{-i j 9.27594} e^{-j 3.79936} \\
& -i \frac{0.81654}{\sqrt{j}} e^{-i 16}(1.5269-i 0.828414)(20.1644-i 2.69787)^{-j / 2} e^{-j 2.83845} e^{-i j 12.5579}
\end{aligned}
$$

Vamos denotar por $a_{k}$ para $k=1,2, \cdots, 24$, a parte das parcelas que não contem j, assim:

$$
\begin{gathered}
a_{1}=-1.044 e^{i 1.03}(1.58428-i 0.764329), \quad a_{2}=-0.901 e^{i 24.2}(1.41326-i 0.0168961), \\
a_{3}=-0.81654 e^{i 16}(1.5269+i 0.828414), \quad a_{4}=-1.15887 e^{i 1.67}(1.6195-i 0.717875), \\
a_{5}=-0.196 e^{i 0.3}(1.45899+i 0.0131905), \quad a_{6}=-0.172579 e^{i 0.14}(1.57736+i 0.778645), \\
a_{7}=i 0.169564 e^{i 3.33}(1.6195-i 0.717875), \quad a_{8}=i 0.196307 e^{i 1.89}(1.45899-i 0.0131905), \\
a_{9}=i 0.12204 e^{i 0.56}(1.57736+i 0.778645), \quad a_{10}=i 0.1984 e^{i 1.67}(1.65211-i 0.650164), \\
a_{11}=i 0.24671 e^{-i 0.1}(1.52852-i 0.00693866), \quad a_{12} i 0.2042 e^{-i 0.0125}(1.6298+i 0.702632), \\
a_{13}=i 0.1984 e^{-i 1.67}(1.65211+i 0.650164), \quad a_{14}=i 0.24671 e^{i 0.1}(1.52852+i 0.00693866), \\
a_{15}=i 0.2042 e^{i 0.0125}(1.6298-i 0.702632), \quad a_{16}=i 0.169564 e^{-i 3.33}(1.6195+i 0.717875), \\
a_{17}=i 0.196307 e^{-i 1.89}(1.45899+i 0.0131905), \quad a_{18}=i 0.12204 e^{-i 0.56}(1.57736-i 0.778645), \\
a_{19}=1.15887 e^{-i 1.67}(1.6195+i 0.717875), \quad a_{20}=0.196 e^{-i 0.3}(1.45899-i 0.0131905), \\
a_{21}=0.172579 e^{-i 0.14}(1.57736-i 0.778645), \quad a_{22}=1.044 e^{-i 1.03}(1.58428+i 0.764329), \\
a_{23}=0.901 e^{-i 24.2}(1.41326+i 0.0168961), \quad a_{24}=0.81654 e^{-i 16}(1.5269-i 0.828414) .
\end{gathered}
$$

Ficando a soma:

$$
\begin{gathered}
Y_{j \lambda_{1} \star \mu_{b}}^{j \mu_{1}} Y_{j \lambda_{2}}^{j \mu_{2}} \approx 0.34685 \frac{e^{\frac{i j 11 \pi}{4}}}{\pi^{2}} \frac{(17.3)^{j}}{2^{2 j}} \\
\left(a_{1} e^{i j 7.3171} e^{-j 3.032}(16.3795+i 0.89136)^{-j / 2}+a_{2} e^{i j 9.27594} e^{-j 3.79936}(17.1925+i 2.52637)^{-j / 2}\right. \\
+a_{3}(20.1644+i 2.69787)^{-j / 2} e^{-j 2.83845} e^{i j 12.5579}+a_{4} e^{i j 6.423079} e^{-j 2.44602}(16.4436+i 0.928146)^{-j / 2}
\end{gathered}
$$


$+a_{5}(17.2989-i 2.39797)^{-j / 2} e^{-j 1.1649} e^{i j 8.36164}+a_{6}(19.9945+i 2.589)^{-j / 2} e^{-j 2.2714} e^{i j 10.30207}$ $+a_{7}(16.4436+i 0.928146)^{-j / 2} e^{-j 2.44602} e^{i j 4.066879}+a_{8}(17.2989+i 2.39797)^{-j / 2} e^{-j 2.868677} e^{i j 8.361626}$ $+a_{9}(19.9945+i 2.589)^{-j / 2} e^{-j 2.2714} e^{i j 9.12397}+a_{10}(16.5263+i 0.985628)^{-j / 2} e^{-j 1.86683} e^{i j(3.1395)}$ $+a_{11}(17.4468+i 2.20811)^{-j / 2} e^{-j 1.97623} e^{i j 4.43417}+a_{12}(19.7645+i 2.46914)^{-j / 2} e^{-j 1.71281} e^{i j 5.72312}$ $+a_{13}(16.5263-i 0.985628)^{-j / 2} e^{-j 1.86683} e^{-i j(3.1395)}+a_{14}(17.4468-i 2.20811)^{-j / 2} e^{-j 1.97623} e^{-i j 4.43417}$ $+a_{15}(19.7645-i 2.46914)^{-j / 2} e^{-j 1.71281} e^{-i j 5.72312}+a_{16}(16.4436-i 0.928146)^{-j / 2} e^{-j 2.44602} e^{-i j 4.066879}$ $+a_{17}(17.2989-i 2.39797)^{-j / 2} e^{-j 2.868677} e^{-i j 8.361626}+a_{18}(19.9945-i 2.589)^{-j / 2} e^{-j 2.2714} e^{-i j 9.12397}$ $+a_{19}(16.4436-i 0.928146)^{-j / 2} e^{-i j 6.423079} e^{-j 2.44602}+a_{20}(17.2989+i 2.39797)^{-j / 2} e^{-j 1.1649} e^{-i j 8.36164}$ $+a_{21}(19.9945-i 2.589)^{-j / 2} e^{-j 2.2714} e^{-i j 10.30207}+a_{22}(16.3795-i 0.89136)^{-j / 2} e^{-i j 7.3171} e^{-j 3.032}$ $\left.+a_{23}(17.1925-i 2.52637)^{-j / 2} e^{-i j 9.27594} e^{-j 3.79936}+a_{24}(20.1644-i 2.69787)^{-j / 2} e^{-j 2.83845} e^{-i j 12.5579}\right)$. Fazendo

$$
\frac{17.3^{j}}{2^{2 j}}=\left(\frac{17.3}{4}\right)^{j}=4.325^{j}=e^{j \ln (4.325)}=e^{j 1.464}
$$

e deixando cada parcela em sua forma polar, obtemos:

$$
\begin{aligned}
& Y_{j \lambda_{1}}^{j \mu_{1}} \star \vec{b} Y_{j \lambda_{2}}^{j \mu_{2}} \approx 0.34685 \frac{e^{i j 8.6394}}{\pi^{2}} e^{j 1.464} \\
& \left(a_{1} e^{i j 7.3171} e^{-j 3.032}\left(16.4037 e^{i 0.0544}\right)^{-j / 2}+a_{2} e^{i j 9.27594} e^{-j 3.79936}\left(17.3771 e^{i 0.145902}\right)^{-j / 2}\right. \\
& +a_{3}\left(20.3441 e^{i 0.1330}\right)^{-j / 2} e^{-j 2.83845} e^{i j 12.5579}+a_{4} e^{i j 6.423079} e^{-j 2.44602}\left(16.4698 e^{i 0.0564}\right)^{-j / 2} \\
& +a_{5}\left(17.4643 e^{-i 0.1377}\right)^{-j / 2} e^{-j 1.1649} e^{i j 8.36164}+a_{6}\left(20.1614 e^{i 0.128769}\right)^{-j / 2} e^{-j 2.2714} e^{i j 10.30207} \\
& +a_{7}\left(16.4698 e^{i 0.0564}\right)^{-j / 2} e^{-j 2.44602} e^{i j 4.066879}+a_{8}\left(17.4643 e^{i 0.1377}\right)^{-j / 2} e^{-j 2.868677} e^{i j 8.361626} \\
& +a_{9}\left(20.1614 e^{i 0.129}\right)^{-j / 2} e^{-j 2.2714} e^{i j 9.12397}+a_{10}\left(16.5557 e^{i 0.06}\right)^{-j / 2} e^{-j 1.86683} e^{i j(3.1395)} \\
& +a_{11}\left(17.586 e^{i 0.126}\right)^{-j / 2} e^{-j 1.97623} e^{i j 4.43417}+a_{12}\left(19.9181 e^{i 0.1243}\right)^{-j / 2} e^{-j 1.71281} e^{i j 5.72312} \\
& +a_{13}\left(16.5557 e^{-i 0.06}\right)^{-j / 2} e^{-j 1.86683} e^{-i j(3.1395)}+a_{14}\left(17.586 e^{-i 0.126}\right)^{-j / 2} e^{-j 1.97623} e^{-i j 4.43417} \\
& +a_{15}\left(19.9181 e^{-i 0.1243}\right)^{-j / 2} e^{-j 1.71281} e^{-i j 5.72312}+a_{16}\left(16.4698 e^{-i 0.0564}\right)^{-j / 2} e^{-j 2.44602} e^{-i j 4.066879} \\
& +a_{17}\left(17.4643 e^{-i 0.136454}\right)^{-j / 2} e^{-j 2.868677} e^{-i j 8.361626}+a_{18}\left(20.1614 e^{-i 0.129}\right)^{-j / 2} e^{-j 2.2714} e^{-i j 9.12397} \\
& +a_{19}\left(16.4698 e^{-i 0.928}\right)^{-j / 2} e^{-i j 6.423079} e^{-j 2.44602}+a_{20}\left(17.4643 e^{i 0.137742}\right)^{-j / 2} e^{-j 1.1649} e^{-i j 8.36164} \\
& +a_{21}\left(20.1614 e^{-i 0.129}\right)^{-j / 2} e^{-j 2.2714} e^{-i j 10.30207}+a_{22}\left(16.4037 e^{-i 0.0544}\right)^{-j / 2} e^{-i j 7.3171} e^{-j 3.032}
\end{aligned}
$$


$\left.+a_{23}\left(17.3771 e^{-i 0.146}\right)^{-j / 2} e^{-i j 9.27594} e^{-j 3.79936}+a_{24}\left(20.3441 e^{-i 0.133}\right)^{-j / 2} e^{-j 2.83845} e^{-i j 12.5579}\right)$.

Arrumando as parcelas, obtemos:

$$
\begin{gathered}
Y_{j \lambda_{1}}^{j \mu_{1}} \star \vec{b} Y_{j \lambda_{2}}^{j \mu_{2}} \approx 0.34685 \frac{e^{i j 8.6394}}{\pi^{2}} \\
+a_{1} 16.4037^{-j / 2} e^{i j 7.3171} e^{-j 1.568} e^{-j i 0.03}+a_{2} 17.3771^{-j / 2} e^{i j 9.27594} e^{-j 2.3324} e^{-i j 0.07} \\
+a_{3} 20.3441^{-j / 2} e^{-i j 0.07} e^{-j 1.37445} e^{i j 12.5579}+a_{4} 16.4698^{-j / 2} e^{i j 6.423079} e^{-j 0.982} e^{-i j 0.03} \\
+a_{5} 17.4643^{-j / 2} e^{-i j 0.07} e^{j 0.2991} e^{i j 8.36164}+a_{6} 20.1614^{-j / 2} e^{-j i 0.06} e^{-j 0.807} e^{i j 10.30207} \\
+a_{7} 16.4698^{-j / 2} e^{-j i 0.03} e^{-j 0.982} e^{i j 4.066879}+a_{8} 17.4643^{-j / 2} e^{-j i 0.07} e^{-j 1.405} e^{i j 8.361626} \\
+a_{9} 20.1614^{-j / 2} e^{-j i 0.065} e^{-j 0.8044} e^{i j 9.12397}+a_{10} 16.5557^{-j / 2} e^{-j i 0.03} e^{-j 0.4028} e^{i j(3.1395)} \\
+a_{11} 17.586^{-j / 2} e^{-j i 0.06} e^{-j 0.51223} e^{i j 4.43417}+a_{12} 19.9181^{-j / 2} e^{-j i 0.062} e^{-j 0.2488} e^{i j 5.72312} \\
+a_{13} 16.5557^{-j / 2} e^{i j 0.03} e^{-j 0.4028} e^{-i j(3.1395)}+a_{14} 17.586^{-j / 2} e^{i j 0.06} e^{-j 0.51223} e^{-i j 4.43417} \\
+a_{15} 19.9181^{-j / 2} e^{i j 0.06} e^{-j 0.2488} e^{-i j 5.72312}+a_{16} 16.4698^{-j / 2} e^{i j 0.03} e^{-j 0.982} e^{-i j 4.066879} \\
+a_{17} 17.4643^{-j / 2} e^{i j 0.07} e^{-j 1.4047} e^{-i j 8.361626}+a_{18} 20.1614^{-j / 2} e^{i j 0.065} e^{-j 0.8074} e^{-i j 9.12397} \\
+a_{19} 16.4698^{-j / 2} e^{i j 0.464} e^{-i j 6.423079} e^{-j 0.982}+a_{20} 17.4643^{-j / 2} e^{-i j 0.07} e^{j 0.2991} e^{-i j 8.36164} \\
+a_{21} 20.1614^{-j / 2} e^{i j 0.065} e^{-j 0.8074} e^{-i j 10.30207}+a_{22} 16.4037^{-j / 2} e^{i j 0.03} e^{-i j 7.3171} e^{-j 1.568} \\
\left.+a_{23} 17.3771^{-j / 2} e^{i j 0.07} e^{-i j 9.27594} e^{-j 2.3354}+a_{24} 20.3441^{-j / 2} e^{i j 0.07} e^{-j 1.37445} e^{-i j 12.5579}\right)
\end{gathered}
$$

E assim, finalmente, absorvendo as constantes em $a_{j}^{\prime} s$ e somando a potência de $e^{i j 8.6394}$ as respectivas potências dentro do parenteses, obtemos:

$$
\begin{aligned}
Y_{j \lambda_{1}}^{j \mu_{1}}{ }_{b} & Y_{j \lambda_{2}}^{j \mu_{2}} \approx\left(a_{1} e^{i j 15.93} e^{-j 2.97}+a_{2} e^{i j 17.85} e^{-j 3.76}+a_{3} e^{i j 21.13} e^{-j 2.88}+a_{4} e^{i j 15.03} e^{-j 2.38}\right. \\
& +a_{5} e^{i j 16.93} e^{-j 1.13}+a_{6} e^{i j 18.88} e^{-j 2.31}+a_{7} e^{i j 12.68} e^{-j 2.38}+a_{8} e^{i j 16.93} e^{-j 2.84} \\
& +a_{9} e^{i j 17.7} e^{-j 2.31}+a_{10} e^{i j 11.75} e^{-j 1.81}+a_{11} e^{i j 13.01} e^{-j 1.95}+a_{12} e^{i j 14.3} e^{-j 1.74} \\
& +a_{13} e^{i j 5.53} e^{-j 1.81}+a_{14} e^{i j 4.27} e^{-j 1.95}+a_{15} e^{i j 2.98} e^{-j 1.74}+a_{16} e^{i j 4.6} e^{-j 2.38} \\
& +a_{17} e^{i j 0.35} e^{-j 2.83}+a_{18} e^{-i j 0.42} e^{-j 2.31}+a_{19} e^{i j 2.68} e^{-j 2.38}+a_{20} e^{i j 0.21} e^{-j 1.13} \\
+ & \left.a_{21} e^{-i j 1.6} e^{-j 2.31}+a_{22} e^{i j 8.42} e^{-j 2.97}+a_{23} e^{-i j 0.57} e^{-j 3.76}+a_{24} e^{-i j 3.85} e^{-j 2.88}\right)
\end{aligned}
$$

Analisando novamente cada parcela, assim como fizemos para o produto de Stratonovich, observemos que o fator $e^{ \pm i j *}$ tem módulo igual a 1, logo, limitada. E os fatores 
que possuem $-j$ tenderão a zero quando $j$ tender a infinito. Portanto ,

$$
Y_{j \lambda_{1}}^{j \mu_{1}}{ }_{b} Y_{j \lambda_{2}}^{j \mu_{2}} \rightarrow 0
$$

Para a análise da "velocidade", tomemos o módulo,

$$
\begin{gathered}
\left|Y_{j \lambda_{1}}^{j \mu_{1}} \star_{b} Y_{j \lambda_{2}}^{j \mu_{2}}\right| \leq\left|a_{1}\right| e^{-j 2.97}+\left|a_{2}\right| e^{-j 3.76}+\left|a_{3}\right| e^{-j 2.88}+\left|a_{4}\right| e^{-j 2.38} \\
+\left|a_{5}\right| e^{-j 1.13}+\left|a_{6}\right| e^{-j 2.31}+\left|a_{7}\right| e^{-j 2.38}+\left|a_{8}\right| e^{-j 2.84}+\left|a_{9}\right| e^{-j 2.31}+\left|a_{10}\right| e^{-j 1.81}+\left|a_{11}\right| e^{-j 1.95}+\left|a_{12}\right| e^{-j 1.74} \\
+\left|a_{13}\right| e^{-j 1.81}+\left|a_{14}\right| e^{-j 1.95}+\left|a_{15}\right| e^{-j 1.74}+\left|a_{16}\right| e^{-j 2.38}+\left|a_{17}\right| e^{-j 2.83}+\left|a_{18}\right| e^{-j 2.31}+\left|a_{19}\right| e^{-j 2.38}+\left|a_{20}\right| e^{-j 1.13} \\
+\left|a_{21}\right| e^{-j 2.31}+\left|a_{22}\right| e^{-j 2.97}+\left|a_{23}\right| e^{-j 3.76}+\left|a_{24}\right| e^{-j 2.88} .
\end{gathered}
$$

Observamos que a parcela de maior potência é a que dominará todas as outras, ou seja,

$$
\left|Y_{j \lambda_{1}}^{j \mu_{1}} \star \vec{b} Y_{j \lambda_{2}}^{j \mu_{2}}\right| \leq M_{0} e^{-1.13 j}
$$

$\operatorname{com} M_{0} \in \mathbb{R}$.

\subsection{Cálculo da aproximação para o produto pontual}

$$
\begin{gathered}
Y_{j \lambda_{1}}^{j \mu_{1}} . Y_{j \lambda_{2}}^{j \mu_{2}} \approx h_{1} j^{5 / 2} \int_{\left|\lambda_{1}-\lambda_{2}\right|}^{\lambda_{\max }} g_{1}\left(e^{i\left(\Sigma_{2}+\Sigma_{3}\right)}+e^{i\left(\Sigma_{2}-\Sigma_{3}\right)}+e^{i\left(\Sigma_{3}-\Sigma_{2}\right)}+e^{-i\left(\Sigma_{2}+\Sigma_{3}\right)}\right) d \lambda \\
\quad+\widetilde{h}_{1} j^{5 / 2} \int_{\left|\lambda_{1}-\lambda_{2}\right|}^{\lambda_{\max }} \widetilde{g}_{1}\left(e^{i\left(\Sigma_{2}+\Sigma_{3}\right)}+e^{i\left(\Sigma_{2}-\Sigma_{3}\right)}+e^{i\left(\Sigma_{3}-\Sigma_{2}\right)}+e^{-i\left(\Sigma_{2}+\Sigma_{3}\right)}\right) d \lambda .
\end{gathered}
$$

Resolveremos cada parcela dessas integrais $R_{i}$ e $\widetilde{R}_{i}$ com a mesma idéia usada para Stratonovich e Berezin.

Comecemos com $R_{1}$ :

$$
R_{1}=e^{i j 3 \pi / 8} \int_{1 / 4}^{3 / 4} g_{1} e^{i j f(\lambda)} d \lambda,
$$

lembrando que $g_{1}=(-1)^{-j \lambda / 2} \sqrt{\frac{\lambda}{\widetilde{\Delta}}}$ e onde

$$
f(\lambda)=\lambda \pi
$$


Para todas as outras parcelas da integral do produto pontual, $R_{2}, R_{3}, R_{4}, \widetilde{R}_{2}, \widetilde{R}_{3}, \widetilde{R}_{4}$, teremos ou a função fase como um fator linear ou como uma constante, em ambas sua derivada será nula e para esse caso, essa integral não fornece uma contribuição. E dai, concluímos que cada parcela será nula. Conluindo ainda que a integral toda será zero. Portanto, nessa aproximação o produto pontual será zero:

$$
Y_{j \lambda_{1}}^{j \mu_{1}} \cdot Y_{j \lambda_{2}}^{j \mu_{2}}=0
$$

\subsection{O método de "Steepest descents"}

Faremos um breve resumo do que esse método faz. Para maiores detalhes ver [1].

Analisando problemas em física matemática, geralmente encontramos o problema de analisar o comportamento de uma função para valores muito grandes, ou seja, o comportamento assintótico da função. Exemplos específicos são fornecidos como a função gamma e as funções de Bessel. O método de "steepest descents"é um método que determina tal comportamento assintótico quando a função pode ser expressada na forma integral:

$$
I(j)=\int_{C} g(z) e^{j \widetilde{f}(z)} d z
$$

Para nosso caso, vamos tomar $j$ real. O contorno de integração $C$ é então escolhido de maneira que a parte real de $f(z)$ aproximade menos infinito em ambos os limites e que o integrando irá se anular nesses limites ou é escolhido como um contorno fechado. Mais ainda, é assumido que $g(z)$ no integrando é dominado pela exponencial na região de interesse.

Se o parametro $j$ é grande e positivo, o valor do integrando será grande quando a parte real de $f(z)$ é grande e será pequeno se a parte real de $f(z)$ for pequena ou negativa. Em particular, se for permitido que $j$ cresça indefinitivamente (levando a dependência assintótica) toda a contribuição do integrando para a integral virá da região onde a parte real de $f(z)$ toma seu valor máximo positivo. Longe desse máximo positivo o integrando será em comparação, insignificantemente pequeno. Isso é visto expressando $f(z)$ como

$$
f(z)=u(x, y)+i v(x, y) .
$$

Daí a integral é escrita como

$$
I(j)=\int_{C} g(z) e^{j u(x, y)} e^{i j v(x, y)} d z .
$$


Se agora, ainda, impusermos que a parte imaginária do expoente $i v(x, y)$ seja constante na região na qual a parte real toma seu máximo valor, isto é, $v(x, y)=v\left(x_{0}, y_{0}\right)=v_{0}$, podemos aproxximar a integral por

$$
I(j) \approx e^{i j v_{0}} \int_{C} g(z) e^{j u(x, y)} d z .
$$

Longe do máximo da parte real, a parte imaginária pode ser permitida oscilatória como se desejar, para o integrando é insignificantemente pequeno e variando o fator fase é portanto irrelevante.

A parte real de $j \widetilde{f}(z)$ é um máximo para um dado $j$ quando a parte real de $\widetilde{f}(z)$, $u(x, y)$, é um máximo. Isto implica que

$$
\frac{\partial u}{\partial x}=\frac{\partial u}{\partial y}=0
$$

e portanto, usando as condições de Cauchy-Riemann, obtemos

$$
\frac{d f(z)}{d z}=0
$$

Vamos continuar para encontrar tais zeros da derivada.

É essencial notar que o valor máximo de $u(x, y)$ é o máximo apenas ao longo de um dado contorno. No plano infinito nem a parte real nem a parte imaginária da nossa função analítica possue um máximo absoluto. Isso pode ser visto relembrando para ambas $u$ e $v$ satisfazem a equação de Laplace,

$$
\frac{\partial^{2} u}{\partial x^{2}}
$$

Disso, se a segunda derivada com respeito a $x$ é positiva, a segunda derivada com respeito a $y$ deve ser negativa, e portanto nem $u$ e nem $v$ podem ter máximos ou mínimos absolutos. Desde que a função $f(z)$ seja tomada analítica, pontos síngulares são claramente excluidos. O anulamento da derivada de $f(z)$ implica que temos um ponto de sela, um valor estacionário, o qual pode ser um máximo de $u(x, y)$ para um contorno e um mínimo para outro.

Nosso problema então é escolher o contorno de integração para satisfazer duas condições:

1. O contorno deve ser escolhido tal que $u(x, y)$ tenha um máximo no ponto de sela. 
2. O contorno deve passar pelo ponto de sela de tal maneira que a parte imaginária $v(x, y)$, seja uma constante.

Essa segunda condição é que leva ao caminho de "steepest descent"e fornece ao método seu nome. Sabemos que as curvas correspondentes a $u=$ constante e $v=$ constante formam um sistema ortogonal. Isto significa que uma curva $v=c_{i}$, contante, é em toda a parte tangente ao gradiente de $u, \nabla u$. Por isso a curva $v=$ contante é a curva que fornece a linha de "steepest descent"a partir do ponto de sela.

No ponto de sela a função $f(z)$ pode ser expandida em série de Taylor e obter

$$
\widetilde{f}(z)=\widetilde{f}\left(z_{0}\right)+\frac{1}{2}\left(z-z_{0}\right)^{2} \widetilde{f}^{\prime \prime}\left(z_{0}\right)+\cdots
$$

A primeira derivada não está, desde que, obviamente, a expressão $d \widetilde{f}(z) / d z=0$. O primeiro termo de correção, $\frac{1}{2}\left(z-z_{0}\right)^{2} \widetilde{f}^{\prime \prime}\left(z_{0}\right)$, é real e negativo. Isto é, é real pois especificamos que a parte imaginária deve ser constante ao londo do nosso contorno e negativo pois estamos movendo do ponto de sela para baixo ou pela "passagem de montanha". Então, assumindo que $f^{\prime \prime}\left(z_{0}\right) \neq 0$, temos

$$
\widetilde{f}(z)-\widetilde{f}\left(z_{0}\right) \approx \frac{1}{2}\left(z-z_{0}\right)^{2} \widetilde{f}^{\prime \prime}\left(z_{0}\right)=-\frac{1}{2 j} t^{2},
$$

que serve para definir uma nova variável $t$. Se $\left(z-z_{0}\right)$ é escrita em forma polar

$$
\left(z-z_{0}\right)=\delta e^{i \alpha}
$$

(com a fase $\alpha$ mantida constante), temos,

$$
t^{2}=-j \widetilde{f}^{\prime \prime}\left(z_{0}\right) \delta^{2} e^{2 i \alpha}
$$

Como $t$ é real, será escrito como $t= \pm \delta\left|j \widetilde{f}^{\prime \prime}\left(z_{0}\right)\right|^{1 / 2}$. Voltando na inegral inicial dessa seção e fazendo as subtituições das funções que analisamos, obtemos:

$$
I(j) \approx g\left(z_{0}\right) e^{j \widetilde{f}\left(z_{0}\right)} \int_{-\infty}^{+\infty} e^{-t^{2} / 2} \frac{d z}{d t} d t
$$

Temos

$$
\frac{d z}{d t}=\left(\frac{d t}{d z}\right)^{-1}=\left(\frac{d t}{d \delta} \frac{d \delta}{d z}\right)^{-1}=\left|j \tilde{f}^{\prime \prime}\left(z_{0}\right)\right|^{-1 / 2} e^{i \alpha}
$$


conlcuimos,

$$
I(j) \approx \frac{g\left(z_{0}\right) e^{j \widetilde{f}\left(z_{0}\right)} e^{i \alpha}}{\left|j \widetilde{f}^{\prime \prime}\left(z_{0}\right)\right|^{1 / 2}} \int_{-\infty}^{+\infty} e^{-t^{2} / 2} d t .
$$

Deve ser observado que os limites de integração são tomados de menos infinto a infinito. Isso é permitido pois o integrando é essencialmente zero quando $t$ se afasta sensívelmente da origem. Note que a integral restante é apenas uma integral Gaussiana igual a $\sqrt{2 \pi}$. E finalmente, obtemos:

$$
I(j) \approx \frac{\sqrt{2 \pi} g\left(z_{0}\right) e^{j \widetilde{f}\left(z_{0}\right)} e^{i \alpha}}{\left|j \widetilde{f}^{\prime \prime}\left(z_{0}\right)\right|^{1 / 2}} .
$$

A fase $\alpha$ introduzida na equação $\left(z-z_{0}\right)=\delta e^{i \alpha}$ como a fase do contorno quando passa pelo ponto de sela. É escolhido de maneira que as duas condições ( $\alpha=$ constant; $\operatorname{Real}[f(z)]=$ maxima ) sejam satisfeitas. Pode acontecer, as vezes, do contorno passar por dois ou mais pontos de sela em sucessão. Se isso acontecer, precisamos apenas adicionar a contribuição dada por a partir de cada ponto de sela em ordem a obter uma aproximação para a integral total.

Um cuidado importante: assumimos que a única contribuição importante para a integral vem de uma vizinhança do ponto de sela $z=z_{0}$, isto é,

$$
\operatorname{Real}[f(z)]=u(x, y) \ll u\left(x_{0}, y_{0}\right)
$$

sobre todo o contorno a partir de $z_{0}=x_{0}+i y_{0}$. 


\section{Apêndice D: Software Mathematica}

Nesse apêndice segue apenas uma das várias contas que fizemos usando o software Mathematica, para que o leitor tenha idéia dos comandos e o que foi feito usando o programa. Frizamos que esse é apenas um pedaço de tudo que tivemos que fazer usando o software. 

$\ln [35]:=$

Quit

$\ln [1]:=$

$11=1 / 2$

$12=1 / 4$

vartheta $=\mathrm{Pi} / 2$

Out[1] $=\frac{1}{2}$

Out[2] $=\frac{1}{4}$

Out[3] $=\frac{\pi}{2}$

$\ln [4]:=m 1=0$

$\mathrm{m} 2=0$

Out $[4]=0$

Out[5]= 0

$\operatorname{In}[6]:=\operatorname{Delta}=\operatorname{Expand}[(11+12+13) *(-11+12+13) *(11-12+13) *(11+12-13)]$

Out $[6]=-\frac{9}{256}+\frac{513^{2}}{8}-13^{4}$

$\ln [7]:=$

Delta123 $=\operatorname{Expand}\left[\left(\operatorname{Delta}-11^{\wedge} 2 * 12^{\wedge} 2 * 13^{\wedge} 2\right)\right]$

Out[7] $=-\frac{9}{256}+\frac{3913^{2}}{64}-13^{4}$

$\ln [8]:=$

teta12 $=\operatorname{ArcSin}\left[\operatorname{Factor}\left[2 *\left(\operatorname{Sqrt}\left[(\operatorname{Delta123}) /\left(\operatorname{Delta} *\left(4-11^{\wedge} 2\right)\right)\right]\right)\right]\right]$

$\operatorname{Out}[8]=\operatorname{ArcSin}\left[\frac{4 \sqrt{\frac{9-15613^{2}+25613^{4}}{9-16013^{2}+25613^{4}}}}{\sqrt{15}}\right]$

$\ln [9]:=$

Solve $\left[\frac{4 \sqrt{\frac{9-15613^{2}+25613^{4}}{9-16013^{2}+25613^{4}}}}{\sqrt{15}}=1,13\right]$

Out[9] $=\left\{\left\{13 \rightarrow-\frac{\sqrt{3}}{4}\right\},\left\{13 \rightarrow \frac{\sqrt{3}}{4}\right\}\right\}$ 
$\ln [10]:=$

Plot [teta12, $\{13,1 / 4,3 / 4\}]$

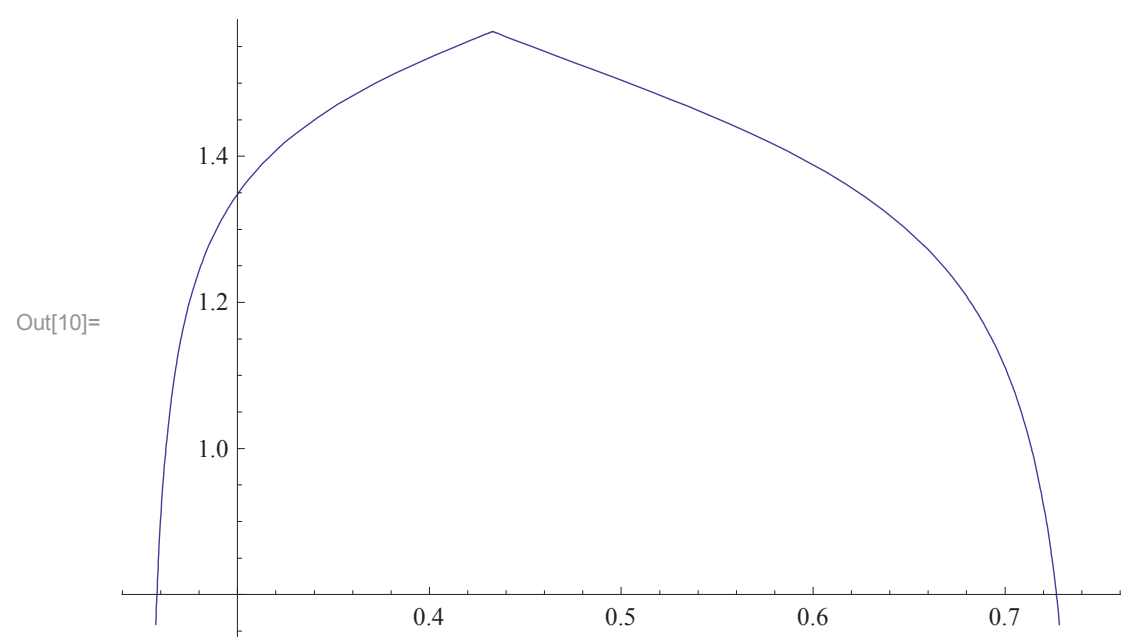

$\ln [11]:=$

Plot $[$ Piecewise $[\{\{$ teta12, $13 \leq \operatorname{Sqrt}[3] / 4\},\{$ Pi-teta12, $13 \geq \operatorname{Sqrt}[3] / 4\}\}]$, $\{13,1 / 4,3 / 4\}]$

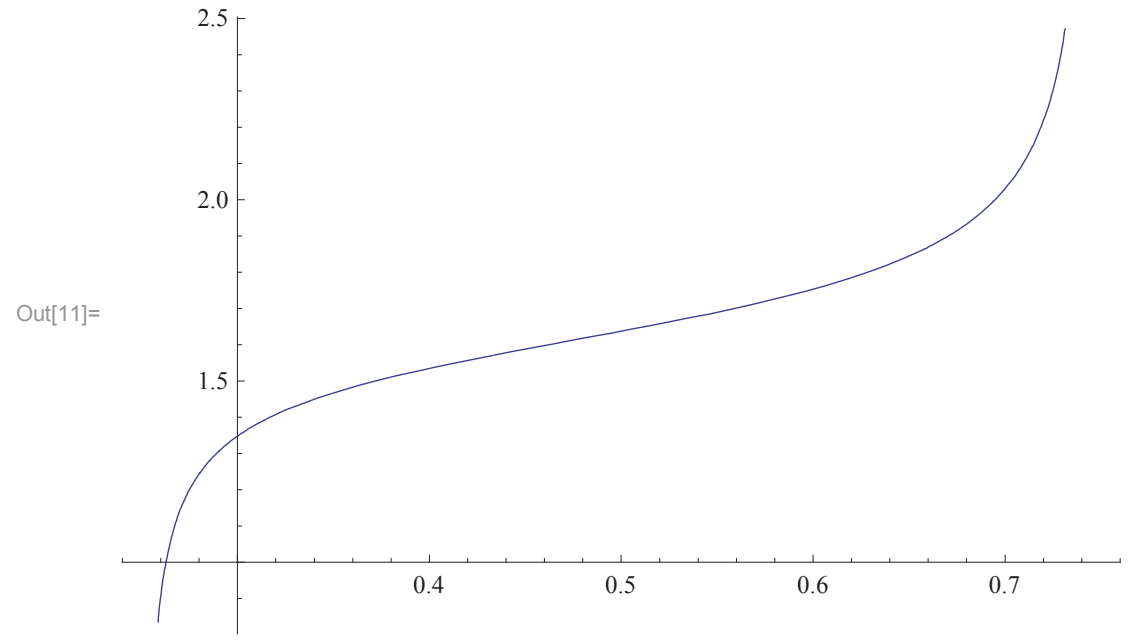

$\ln [12]:=$

$\operatorname{teta13}=\operatorname{ArcSin}\left[\operatorname{Factor}\left[2 *\left(\operatorname{Sqrt}\left[(\operatorname{Delta123}) /\left(\operatorname{Delta} *\left(4-12^{\wedge} 2\right)\right)\right]\right)\right]\right]$

$\operatorname{Out[12]}=\operatorname{Arcsin}\left[\frac{8 \sqrt{\frac{9-15613^{2}+25613^{4}}{9-16013^{2}+25613^{4}}}}{3 \sqrt{7}}\right]$ 
$\ln [13]:=$

Plot $[$ teta13, $\{13,1 / 4,3 / 4\}]$

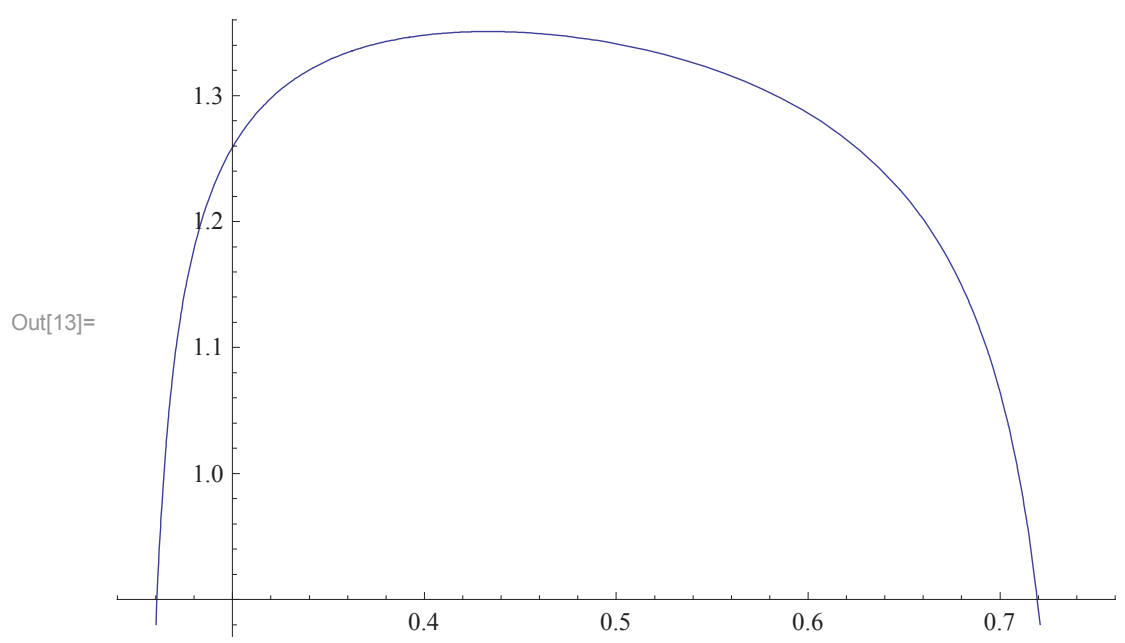

$\ln [14]:=$

teta14 $=\operatorname{ArcSin}\left[\operatorname{Factor}\left[2 *\left(\operatorname{Sqrt}\left[(\operatorname{Delta123}) /\left(\operatorname{Delta} *\left(4-13^{\wedge} 2\right)\right)\right]\right)\right]\right]$

Out[14] $=\operatorname{ArcSin}\left[2 \sqrt{\frac{-9+15613^{2}-25613^{4}}{-36+64913^{2}-118413^{4}+25613^{6}}}\right]$

$\ln [15]:=$

Solve $\left[2 \sqrt{ }\left(\left(-9+15613^{2}-25613^{4}\right) /\left(-36+64913^{2}-118413^{4}+25613^{6}\right)\right)=1,13\right]$

$\operatorname{Out}[15]=\left\{\{13 \rightarrow 0\},\left\{13 \rightarrow-\frac{\sqrt{5}}{4}\right\},\left\{13 \rightarrow \frac{\sqrt{5}}{4}\right\}\right\}$

$\ln [16]:=\operatorname{Plot}[\operatorname{teta14},\{13,1 / 4,3 / 4\}]$

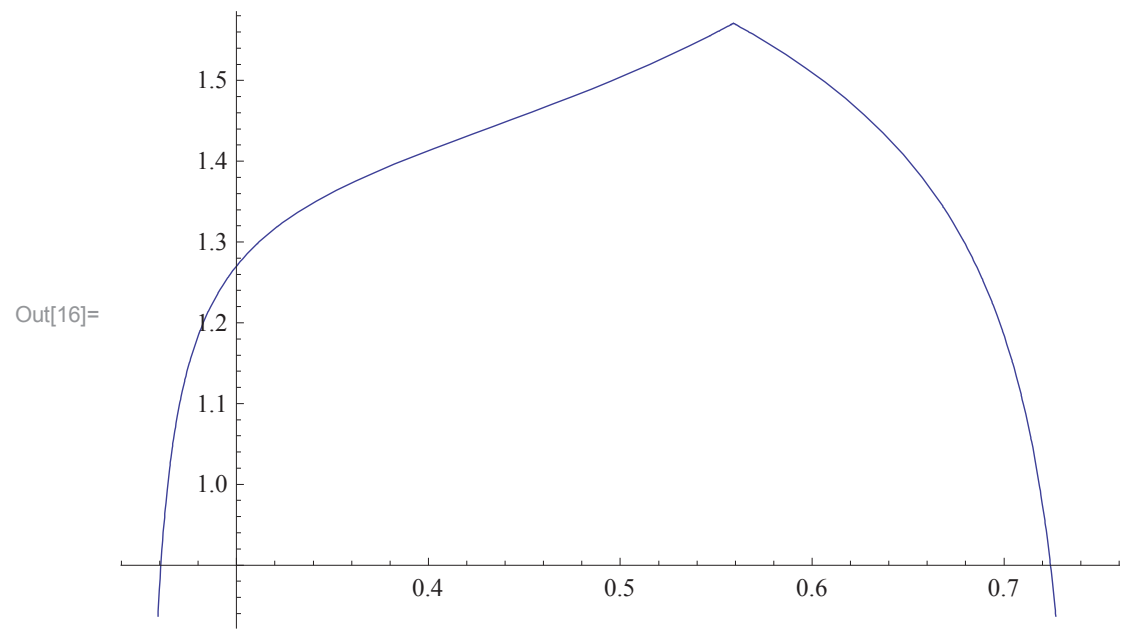


$\operatorname{In}[17]:=\operatorname{Plot}[\mathrm{Piecewise}[\{\{$ teta14, $13 \leq \operatorname{Sqrt}[5] / 4\},\{\mathrm{Pi}-\operatorname{teta14}, 13 \geq \operatorname{Sqrt}[5] / 4\}\}]$, $\{13,1 / 4,3 / 4\}]$

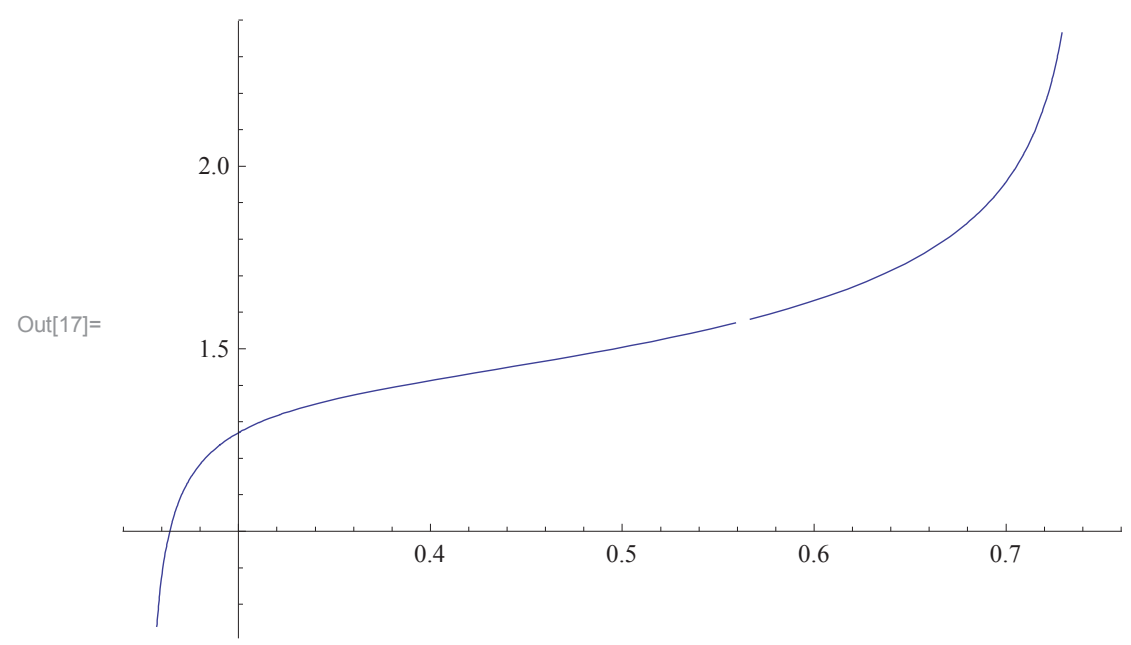

$\ln [18]:=$

tetalambda1 $=\operatorname{ArcCos}\left[\left(2 * 11^{\wedge} 2 *(\mathrm{~m} 1+\mathrm{m} 2)-\mathrm{m} 1 *\left(11^{\wedge} 2-12^{\wedge} 2+13^{\wedge} 2\right)\right) /\right.$

$\left.\left(\operatorname{Sqrt}\left[\left(\left(11^{\wedge} 2-\mathrm{ml}^{\wedge} 2\right) *\left(4 * 11^{\wedge} 2 * 13^{\wedge} 2-\left(11^{\wedge} 2-12^{\wedge} 2+13^{\wedge} 2\right)^{\wedge} 2\right)\right)\right]\right)\right]$

Out[18] $=\frac{\pi}{2}$

$\operatorname{In}[19]:=\operatorname{tetalambda} 2=\operatorname{ArcCos}\left[\left(2 * 12^{\wedge} 2 * \mathrm{~m} 1-\mathrm{m} 2 *\left(11^{\wedge} 2+12^{\wedge} 2-13^{\wedge} 2\right)\right) /\right.$

$\left.\left(\operatorname{Sqrt}\left[\left(\left(12^{\wedge} 2-\mathrm{m} 2^{\wedge} 2\right) *\left(4 * 11^{\wedge} 2 * 12^{\wedge} 2-\left(11^{\wedge} 2+12^{\wedge} 2-13^{\wedge} 2\right)^{\wedge} 2\right)\right)\right]\right)\right]$

Out[19] $=\frac{\pi}{2}$

$\ln [20]:=$

tetalambda $3=\operatorname{ArcCos}\left[\left(2 * 13^{\wedge} 2 * m 2+(m 1+m 2) *\left(-11^{\wedge} 2+12^{\wedge} 2+13^{\wedge} 2\right)\right) /\right.$

$\left.\left(\operatorname{Sqrt}\left[\left(\left(13^{\wedge} 2-(\mathrm{m} 1+\mathrm{m} 2)^{\wedge} 2\right) *\left(4 * 12^{\wedge} 2 * 13^{\wedge} 2-\left(-11^{\wedge} 2+12^{\wedge} 2+13^{\wedge} 2\right)^{\wedge} 2\right)\right)\right]\right)\right]$

Out[20] $=\frac{\pi}{2}$

$\ln [21]:=$

teta $34=$

$\operatorname{ArcSin}\left[\right.$ Factor $\left.\left[(2 * \operatorname{Sqrt}[\operatorname{Delta123}]) /\left(12 * 13 * \operatorname{Sqrt}\left[\left(4-12^{\wedge} 2\right) *\left(4-13^{\wedge} 2\right)\right]\right)\right]\right]$

$\operatorname{Out}[21]=\operatorname{ArcSin}\left[\frac{2 \sqrt{-9+15613^{2}-25613^{4}}}{3 \sqrt{7} 13 \sqrt{4-13^{2}}}\right]$

$\ln [22]:=$

Solve $\left[\frac{2 \sqrt{-9+15613^{2}-25613^{4}}}{3 \sqrt{7} 13 \sqrt{4-13^{2}}}=1,13\right]$

Out[22] $=\left\{\left\{13 \rightarrow \sqrt{\frac{6}{31}}\right\}\right\}$ 
$\ln [23]:=$

Plot $[$ teta34, $\{13,1 / 4,3 / 4\}]$

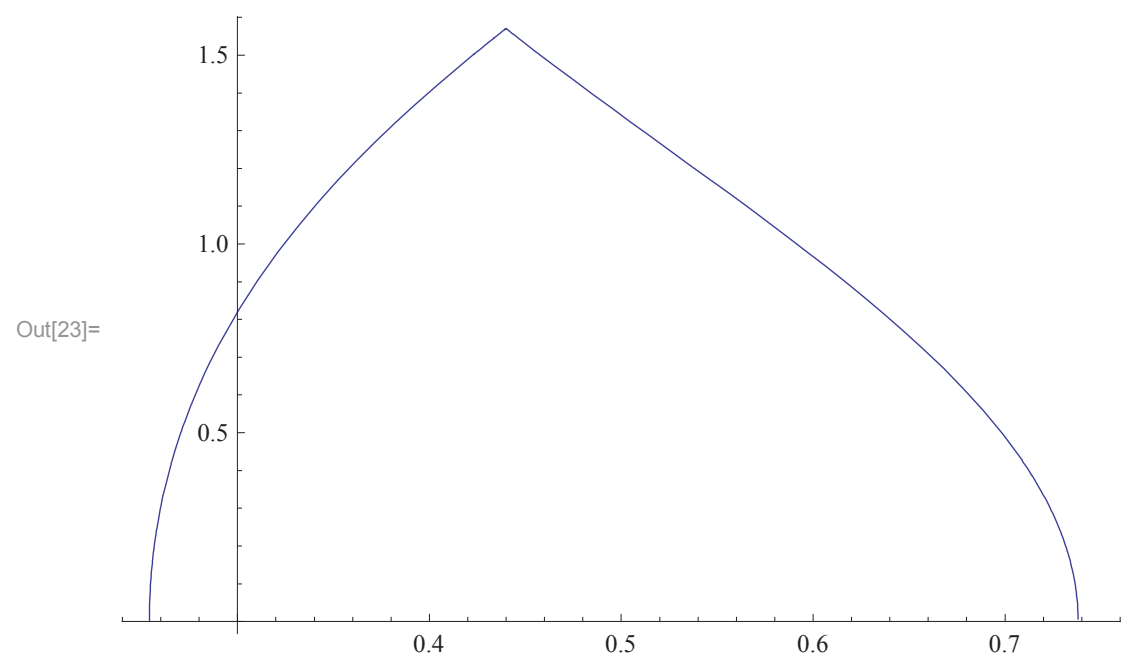

$\ln [24]:=$

Plot $[$ Piecewise $[\{\{$ teta34, $13 \leq \operatorname{Sqrt}[6 / 31]\},\{P i-\operatorname{teta} 34,13 \geq \operatorname{Sqrt}[6 / 31]\}\}]$, $\{13,1 / 4,3 / 4\}]$

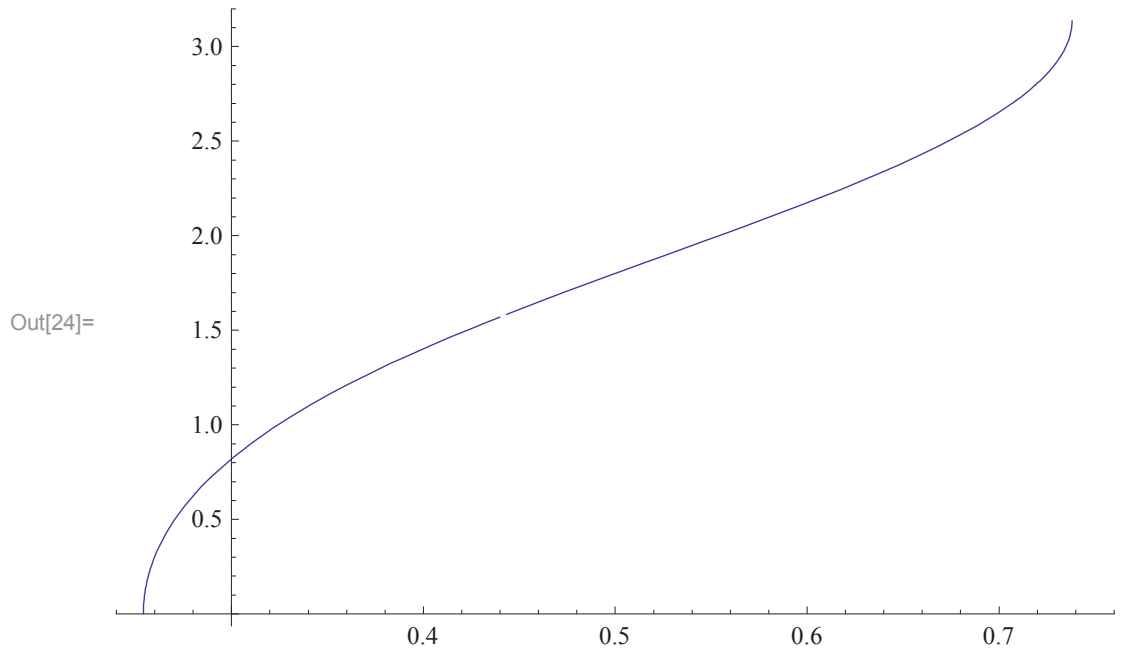

$\ln [25]:=$

teta24 =

$\operatorname{ArcSin}\left[\right.$ Factor $\left.\left[(2 * \operatorname{Sgrt}[\operatorname{Delta123}]) /\left(11 * 13 * \operatorname{Sgrt}\left[\left(4-11^{\wedge} 2\right) *\left(4-13^{\wedge} 2\right)\right]\right)\right]\right]$

Out[25] $=\operatorname{Arcsin}\left[\frac{\sqrt{-9+15613^{2}-25613^{4}}}{2 \sqrt{15} 13 \sqrt{4-13^{2}}}\right]$ 
6 | plotando_grafico_funcao_fase_colocar na tese_apendice_B.nb

$\ln [26]:=$

Plot [teta24, $\{13,1 / 4,3 / 4\}$ ]

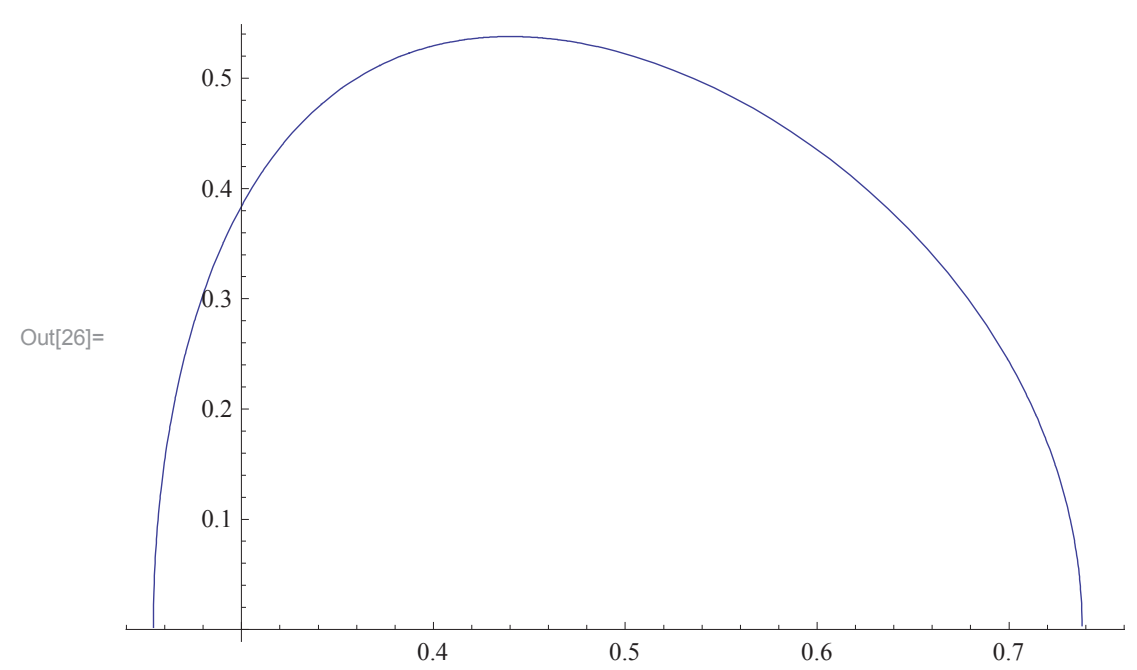

$\ln [27]:=$

teta23 =

$\operatorname{ArcSin}\left[\right.$ Factor $\left.\left[(2 * \operatorname{Sqrt}[\operatorname{Delta123}]) /\left(11 * 12 * \operatorname{Sqrt}\left[\left(4-11^{\wedge} 2\right) *\left(4-12^{\wedge} 2\right)\right]\right)\right]\right]$

Out[27]= $\operatorname{ArcSin}\left[\frac{8 \sqrt{-9+15613^{2}-25613^{4}}}{3 \sqrt{105}}\right]$

$\ln [28]:=$

Solve $\left[\frac{8 \sqrt{-9+15613^{2}-25613^{4}}}{3 \sqrt{105}}=1,13\right]$

Out[28] $=\left\{\left\{13 \rightarrow-\frac{\sqrt{\frac{39}{2}}}{8}\right\},\left\{13 \rightarrow \frac{\sqrt{\frac{39}{2}}}{8}\right\}\right\}$

$\ln [29]:=$

Plot [teta23, $\{13,1 / 4,3 / 4\}]$

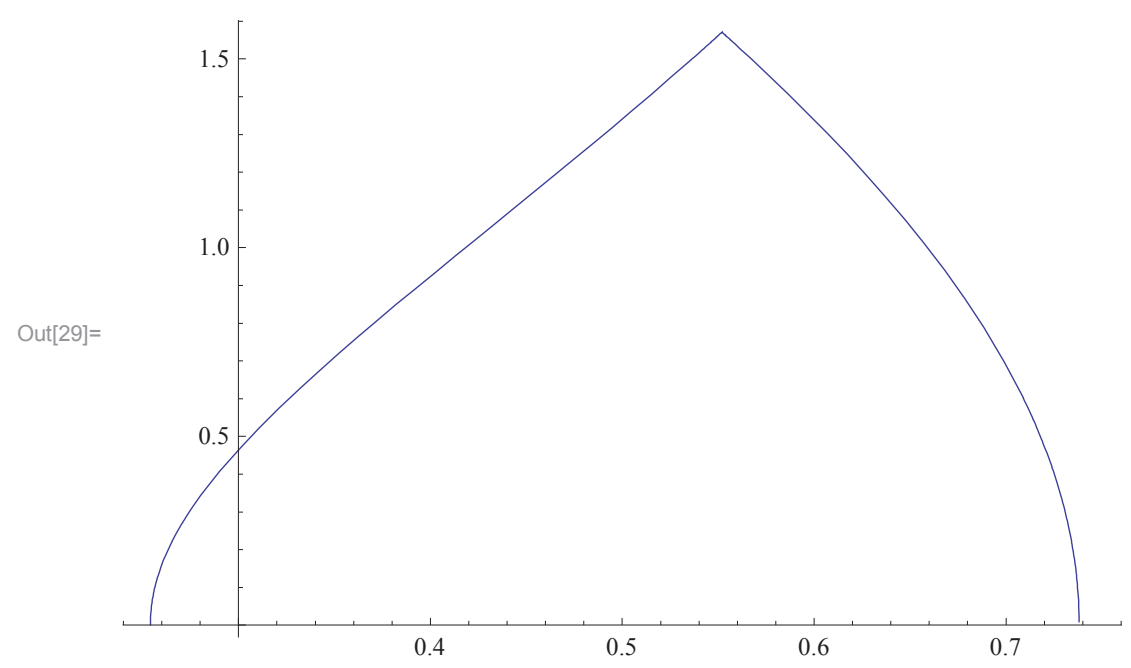


$\ln [40]:=$

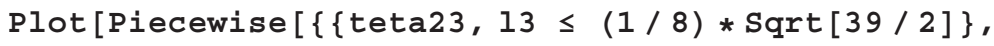

$\{\mathrm{Pi}-\operatorname{teta23}, 13 \geq(1 / 8) * \operatorname{Sqrt}[39 / 2]\}\}],\{13,1 / 4,3 / 4\}]$

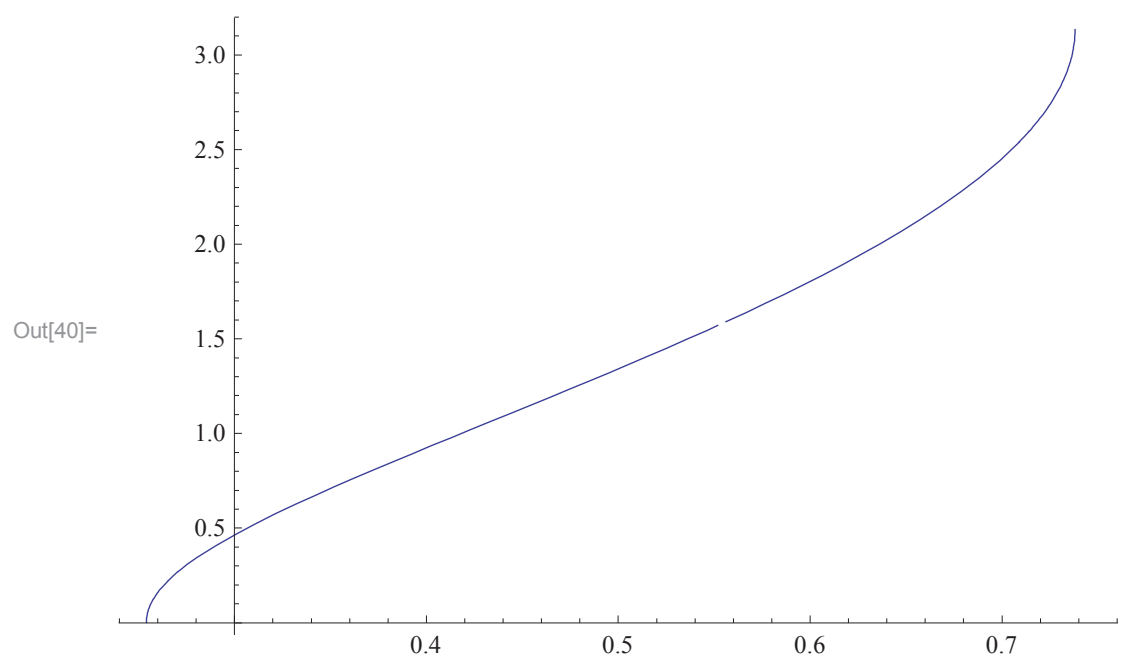

$\ln [41]:=$

anguloD $=\operatorname{ArcCos}\left[\left(11^{\wedge} 2-12^{\wedge} 2-13^{\wedge} 2+2 * \mathrm{~m} 2 *(\mathrm{~m} 1+\mathrm{m} 2)\right) /\right.$

$\left.\left(2 *\left(\operatorname{Sqrt}\left[\left(12^{\wedge} 2-\mathrm{m}^{\wedge} 2\right) *\left(13^{\wedge} 2-(\mathrm{m} 1+\mathrm{m} 2)^{\wedge} 2\right)\right]\right)\right)\right]$

$\operatorname{Out}[41]=\operatorname{ArcCos}\left[\frac{2\left(\frac{3}{16}-13^{2}\right)}{\sqrt{13^{2}}}\right]$

$\ln [42]:=$

Plot [anguloD, $\{13,1 / 4,3 / 4\}]$

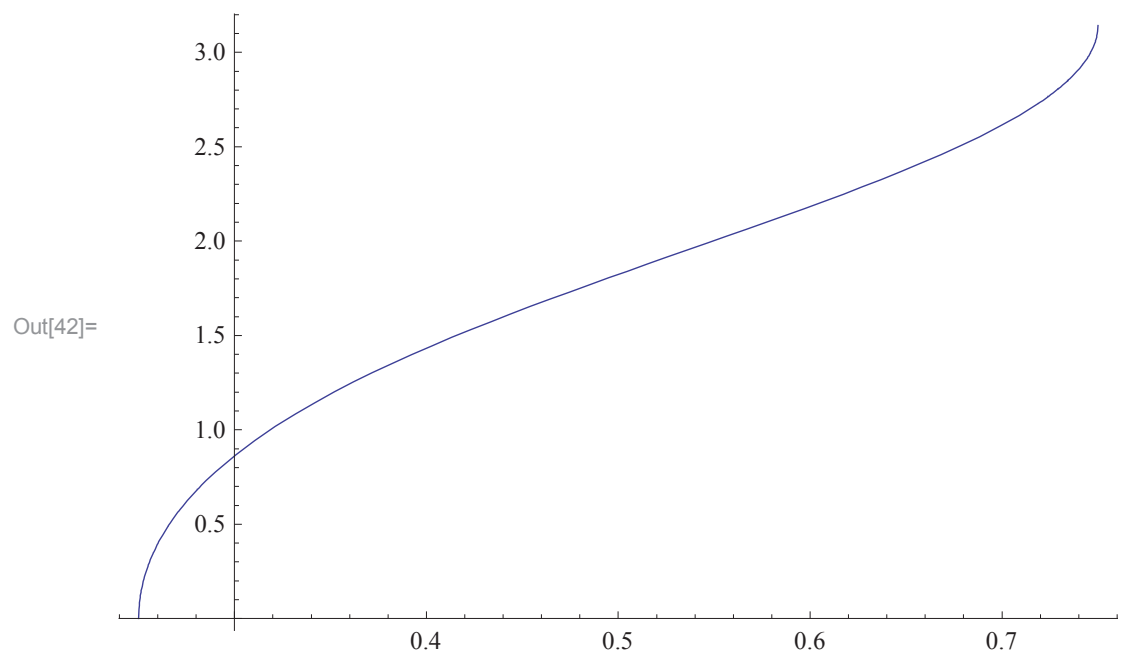

$\ln [43]:=$

anguloE $=\operatorname{ArcCos}\left[\left(-11^{\wedge} 2+12^{\wedge} 2-13^{\wedge} 2+2 * \mathrm{~m} 1 *(\mathrm{~m} 1+\mathrm{m} 2)\right) /\right.$

$\left.\left(2 *\left(\operatorname{Sqrt}\left[\left(11^{\wedge} 2-m 1^{\wedge} 2\right) *\left(13^{\wedge} 2-(m 1+m 2)^{\wedge} 2\right)\right]\right)\right)\right]$

$\operatorname{Out}[43]=\operatorname{ArcCos}\left[\frac{-\frac{3}{16}-13^{2}}{\sqrt{13^{2}}}\right]$ 
$\ln [44]:=$

Plot [anguloE, $\{13,1 / 4,3 / 4\}]$

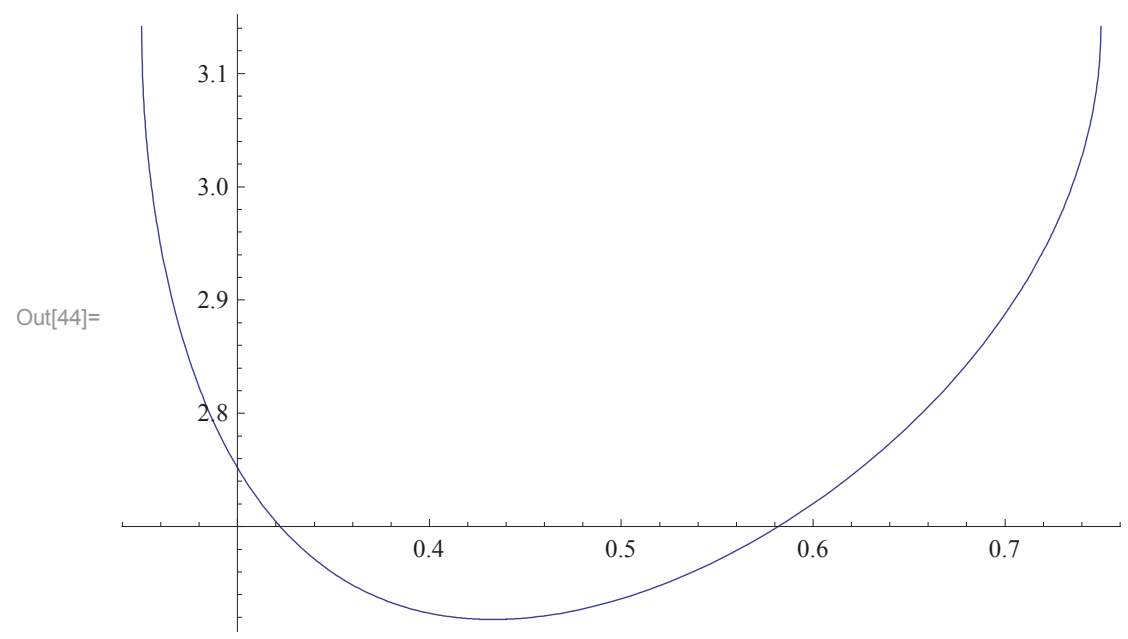

$\operatorname{In}[45]:=$ Deltatil $=$ Expand $\left[\left(11^{\wedge} 2-\mathrm{m}^{\wedge} 2\right) *\left(-11^{\wedge} 2+\mathrm{m} 1^{\wedge} 2+12^{\wedge} 2-\mathrm{m} 2{ }^{\wedge} 2+13^{\wedge} 2-(\mathrm{m} 1+\mathrm{m} 2)^{\wedge} 2\right)+\right.$ $\left(12^{\wedge} 2-m 2^{\wedge} 2\right) *\left(11^{\wedge} 2-m 1^{\wedge} 2-12^{\wedge} 2+m 2^{\wedge} 2+13^{\wedge} 2-(m 1+m 2)^{\wedge} 2\right)+$

$\left.\left(13^{\wedge} 2-(m 1+m 2)^{\wedge} 2\right) *\left(11^{\wedge} 2-m 1^{\wedge} 2+12^{\wedge} 2-m 2^{\wedge} 2-13^{\wedge} 2+(m 1+m 2)^{\wedge} 2\right)\right]$

Out $[45]=-\frac{9}{256}+\frac{513^{2}}{8}-13^{4}$

$\ln [46]:=$

Factor [Deltatil * Delta123]

Out[46] $]=\frac{(-3+413)(-1+413)(1+413)(3+413)\left(9-15613^{2}+25613^{4}\right)}{65536}$

$\ln [47]:=$

g1 $=\left((-1)^{\wedge}(j * 13) * \operatorname{Sqrt}[13]\right) /$

Factor $\left[\left(\left(\text { Delta }-11^{\wedge} 2 * 12^{\wedge} 2 * 13^{\wedge} 2\right) *(\text { Deltatil })\right)\right]^{\wedge}(1 / 4)$

$\operatorname{Out}[47]=\frac{16(-1)^{j 13} \sqrt{13}}{\left((-3+413)(-1+413)(1+413)(3+413)\left(9-15613^{2}+25613^{4}\right)\right)^{1 / 4}}$

$\ln [48]:=$ flambda $=$

$11 *$ teta $12+12 *$ teta $13+13 *$ teta $14+$ teta $34+$ teta $24+$ teta $23+11 *$ tetalambda $1+$ 12 * tetalambda $2+13$ * tetalambda $3+\mathrm{m} 2$ * anguloD $-\mathrm{m} 1$ * anguloE +13 * vartheta; 
Plot [flambda, $\{13,1 / 4, \operatorname{Sqrt}[6 / 31]\}]$

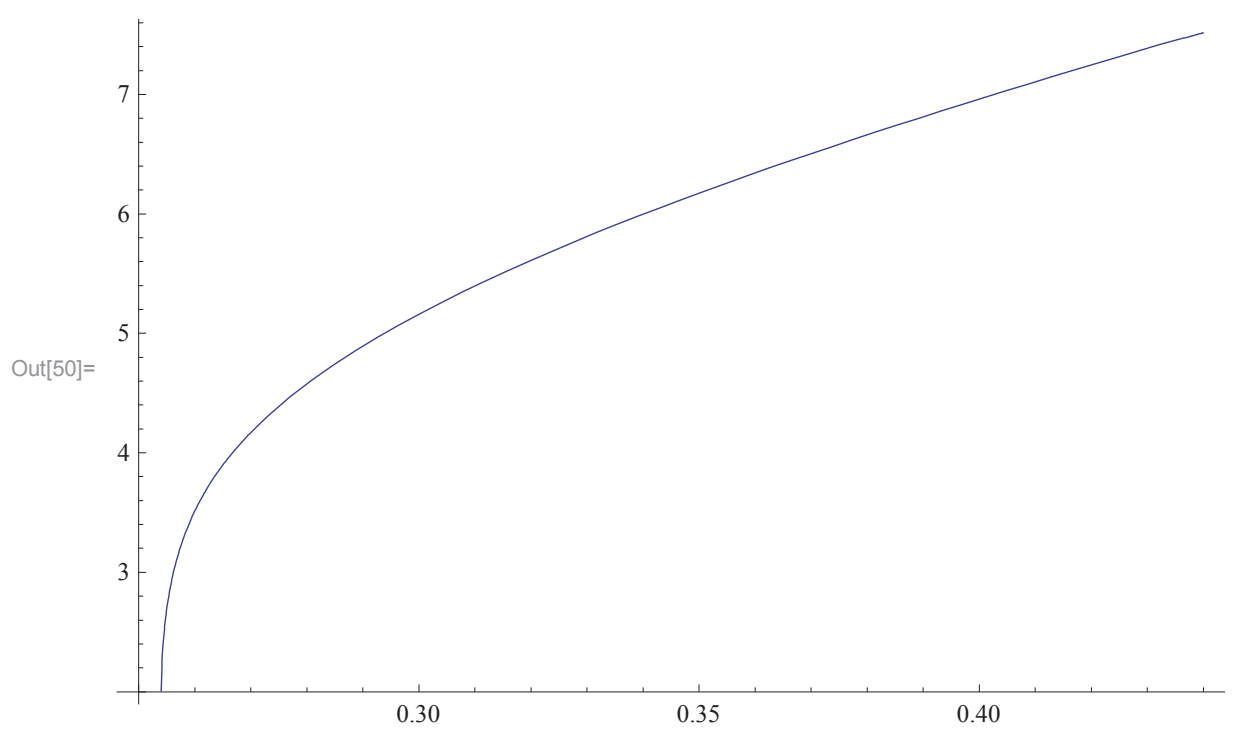

$\operatorname{In}[51]:=$

flambdall $=11 *(P i-$ teta 12$)+12 *$ teta $13+13 *$ teta $14+(P i-$ teta 34$)+$ teta $24+(P i-$ teta23 $)+11 *$ tetalambda $1+12 *$ tetalambda $2+$ 13 * tetalambda $3+\mathrm{m} 2$ * anguloD $-\mathrm{m} 1$ * anguloE +13 * vartheta ;

$\ln [52]:=$

flambda2 $2=11 *(P i-$ teta 12$)+12 *$ teta $13+13 *$ teta $14+$ $($ teta 34$)+$ teta $24+($ teta23 $)+11 *$ tetalambda $1+12$ *tetalambda $2+$ $13 *$ tetalambda $3+\mathrm{m} 2$ * anguloD $-\mathrm{m} 1$ * anguloE +13 * vartheta;

$\ln [53]:=$

flambda3 $3=11 *(P i-$ teta 12$)+12 *$ teta $13+13 *$ teta $14+$

$(\mathrm{Pi}-$ teta34) + teta2 4 + (teta23) + 11 *tetalambda $1+12$ *tetalambda $2+$

$13 *$ tetalambda $3+\mathrm{m} 2$ * anguloD $-\mathrm{m} 1$ * anguloE +13 * vartheta;

$\ln [54]:=$

flambda44 $=11 *(P i-$ teta12 $)+12 *$ teta13 $+13 *(P i-$ teta14 $)+$

$(\mathrm{Pi}-$ teta34 $)+$ teta $24+(\mathrm{Pi}-$ teta23 $)+11 *$ tetalambda $1+12 *$ tetalambda $2+$

13 * tetalambda $3+\mathrm{m} 2$ * anguloD $-\mathrm{m} 1$ * anguloE +13 * vartheta ; 


\section{$\operatorname{In}[55]:=$ Plot $[$ Piecewise [}

$\{\{f l a m b d a, 1 / 4 \leq 13 \leq \operatorname{Sqrt}[3] / 4\},\{f l a m b d a 22, \operatorname{Sqrt}[3] / 4 \leq 13 \leq \operatorname{Sqrt}[6 / 31]\}$, $\{f l a m b d a 33, \operatorname{Sqrt}[6 / 31] \leq 13 \leq(1 / 8) * \operatorname{Sqrt}[39 / 2]\}$, $\{f l a m b d a 11,(1 / 8) * \operatorname{Sqrt}[39 / 2] \leq 13 \leq \operatorname{Sqrt}[5] / 4\}$, $\{f l a m b d a 44, \operatorname{Sqrt}[5] / 4 \leq 13 \leq 3 / 4\}\}],\{13,1 / 4,3 / 4\}]$

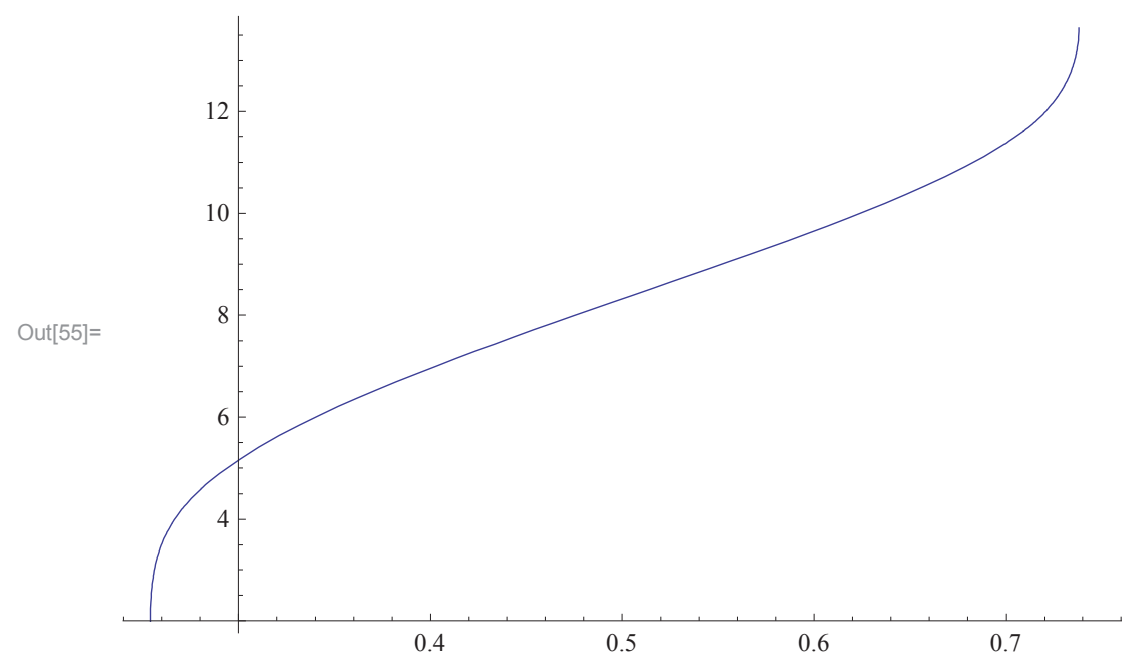




\section{Quit}

$11=1 / 2$

$12=1 / 4$

vartheta $=\mathrm{Pi} / 2$

$\frac{1}{2}$
$\frac{1}{4}$
$\frac{\pi}{2}$

$\mathrm{m} 1=0$

$\mathrm{m} 2=0$

0

0

Delta $=$ Expand $[(11+12+13) *(-11+12+13) *(11-12+13) *(11+12-13)]$

$-\frac{9}{256}+\frac{513^{2}}{8}-13^{4}$

Delta123 $=$ Expand $\left[\left(\right.\right.$ Delta $\left.\left.-11^{\wedge} 2 * 12^{\wedge} 2 * 13^{\wedge} 2\right)\right]$

$-\frac{9}{256}+\frac{3913^{2}}{64}-13^{4}$

h12 $=2$ * Factor $\left[\left(\operatorname{Sqrt}\left[(\operatorname{Delta123}) /\left(\operatorname{Delta} *\left(4-11^{\wedge} 2\right)\right)\right]\right)\right]$

teta12 = ArcSin [h12]

$\frac{4 \sqrt{\frac{9-15613^{2}+25613^{4}}{9-16013^{2}+25613^{4}}}}{\sqrt{15}}$

$\operatorname{ArcSin}\left[\frac{4 \sqrt{\frac{9-15613^{2}+25613^{4}}{9-16013^{2}+25613^{4}}}}{\sqrt{15}}\right]$ 


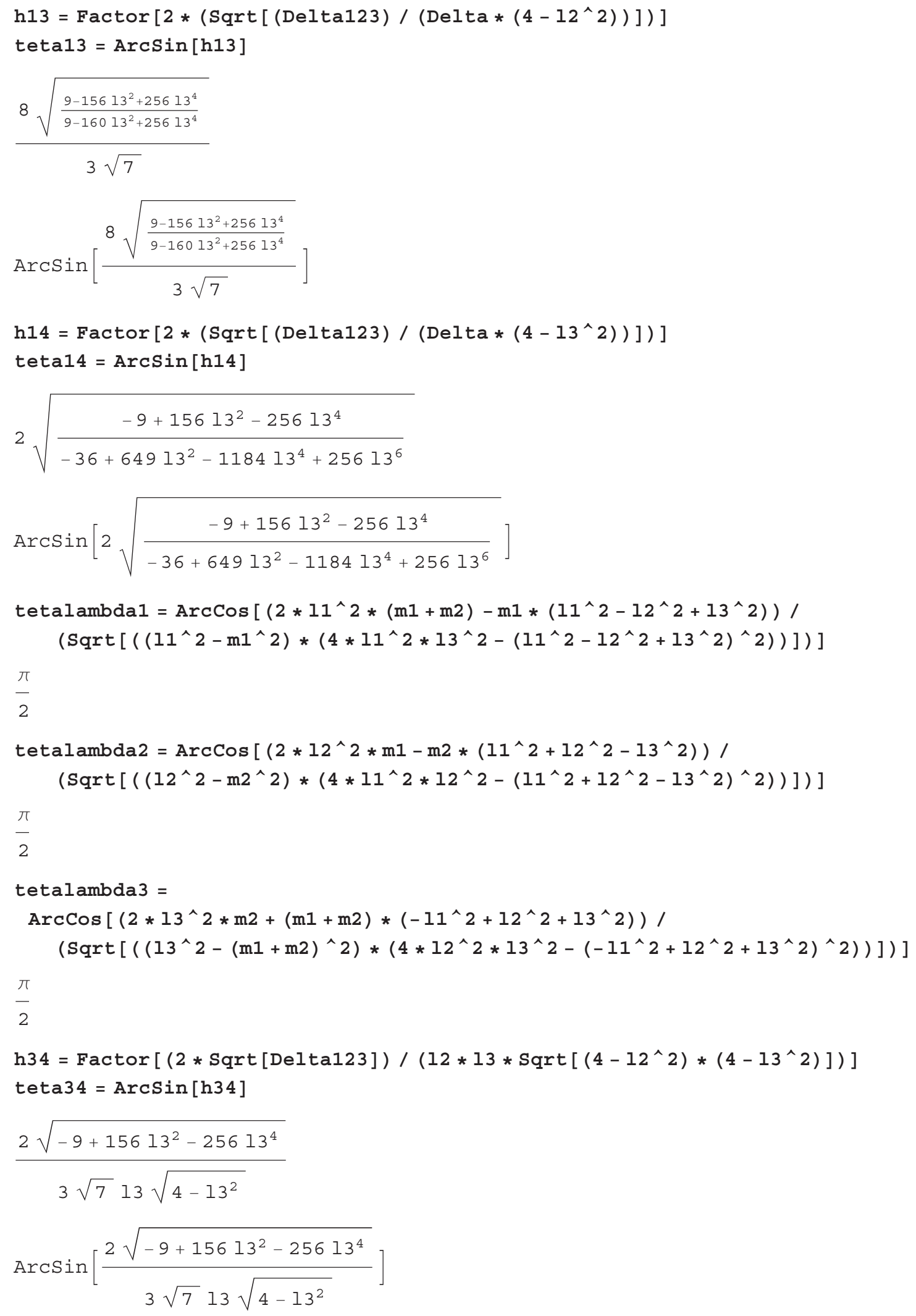




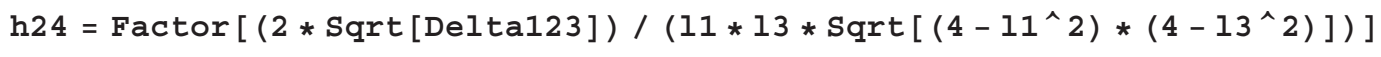




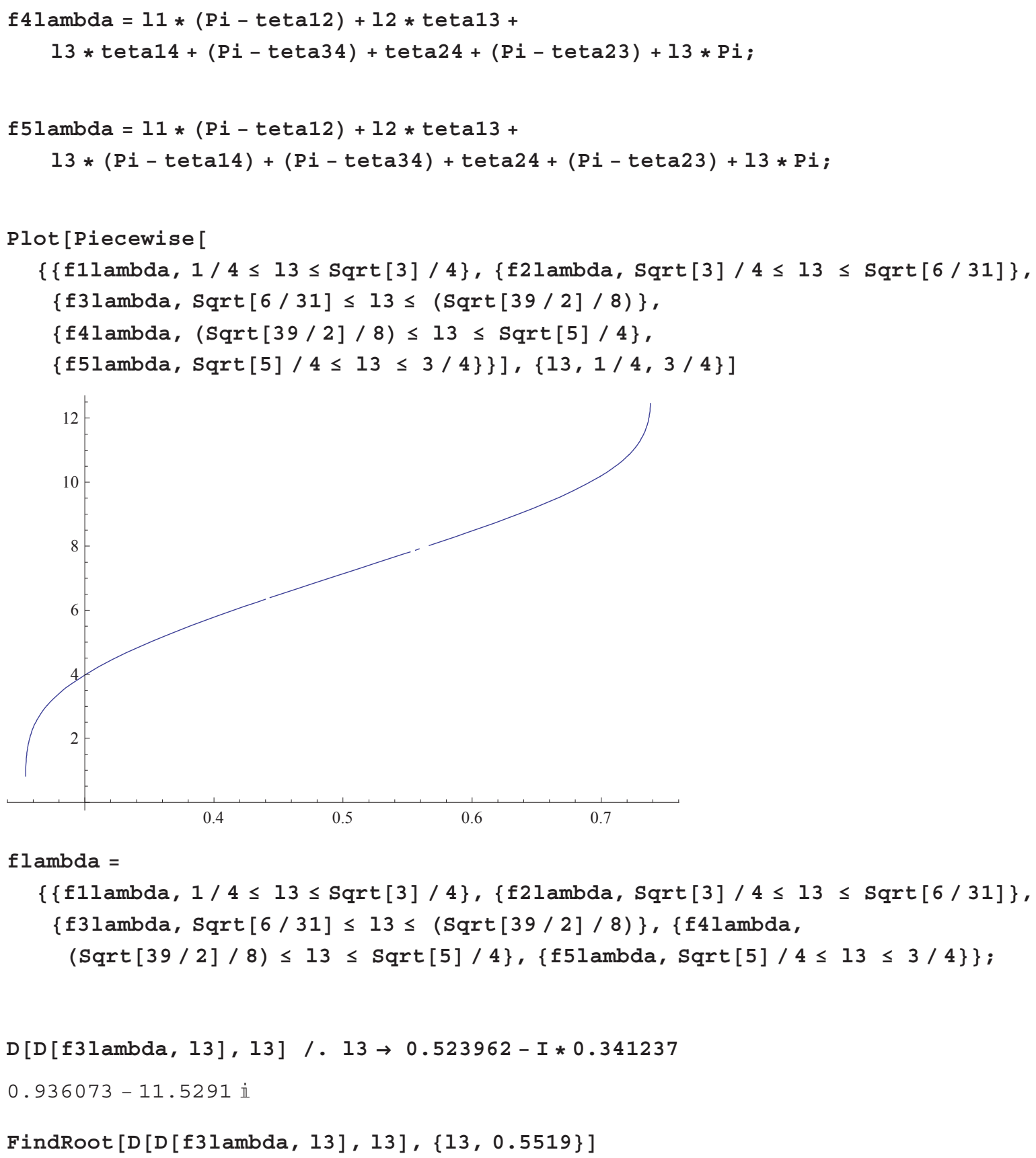

flambda $=$

$\{\{$ f1lambda, $1 / 4 \leq 13 \leq \operatorname{Sqrt}[3] / 4\},\{$ f2lambda, Sqrt [3] / $4 \leq 13 \leq \operatorname{Sqrt}[6 / 31]\}$, $\{f 3 l$ ambda, Sqrt $[6 / 31] \leq 13 \leq(\operatorname{Sqrt}[39 / 2] / 8)\},\{f 4$ lambda,

$(\operatorname{Sqrt}[39 / 2] / 8) \leq 13 \leq \operatorname{Sqrt}[5] / 4\},\{$ f5lambda, Sqrt[5] /4 $\leq 13 \leq 3 / 4\}\}$;

$D[D[f 3 l a m b d a, 13], 13] / .13 \rightarrow 0.523962-I * 0.341237$

$0.936073-11.5291 \dot{1}$

FindRoot $[D[D[f 31$ ambda, 13$], 13],\{13,0.5519\}]$

$\{13 \rightarrow 0.509677\}$

Series $[f 31$ ambda, $\{13,0.509677,7\}]$

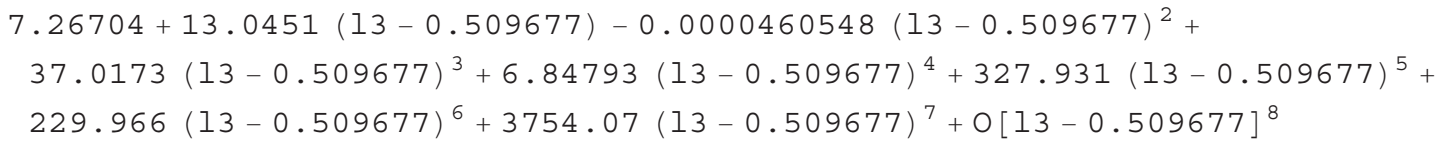




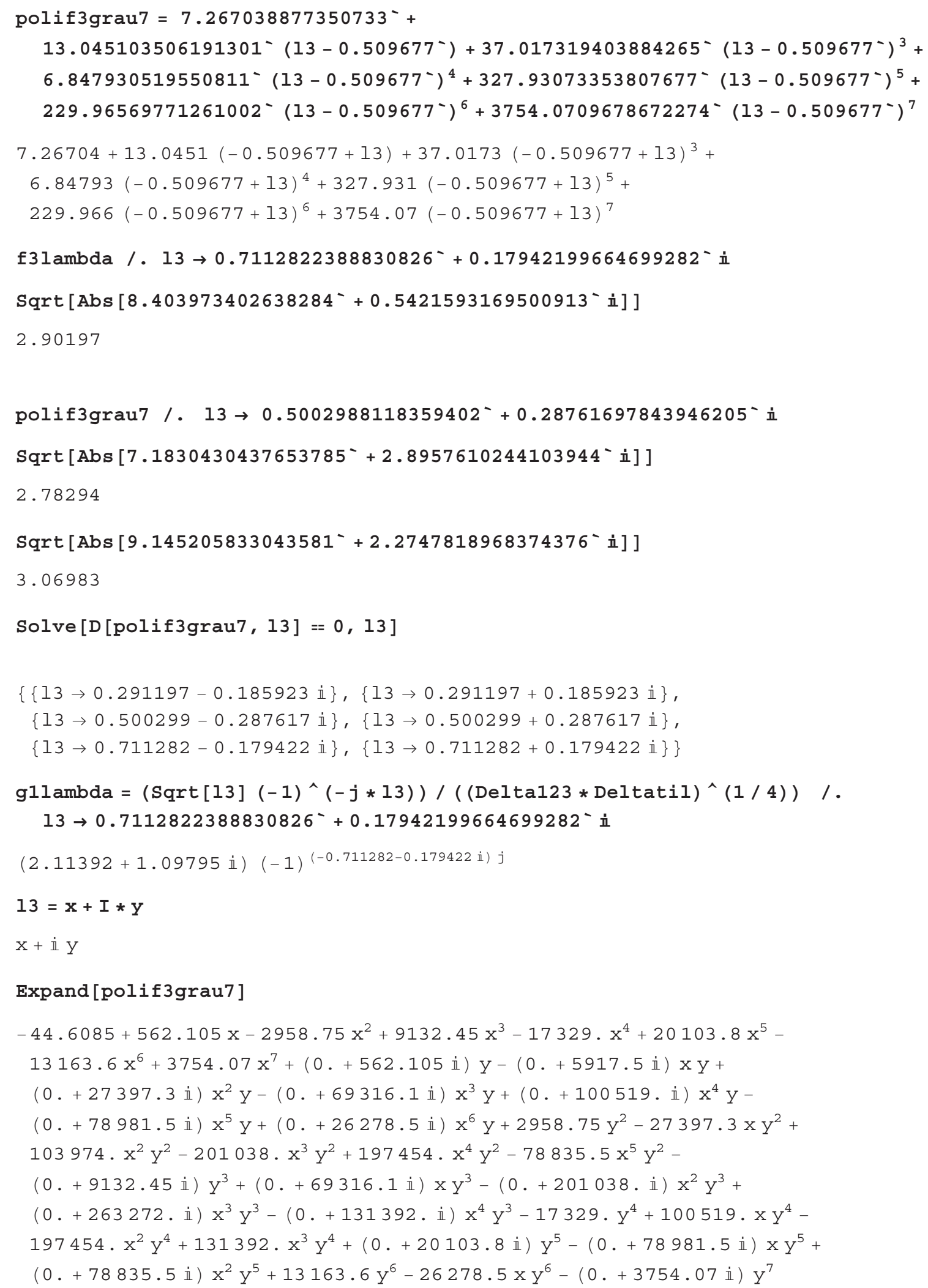




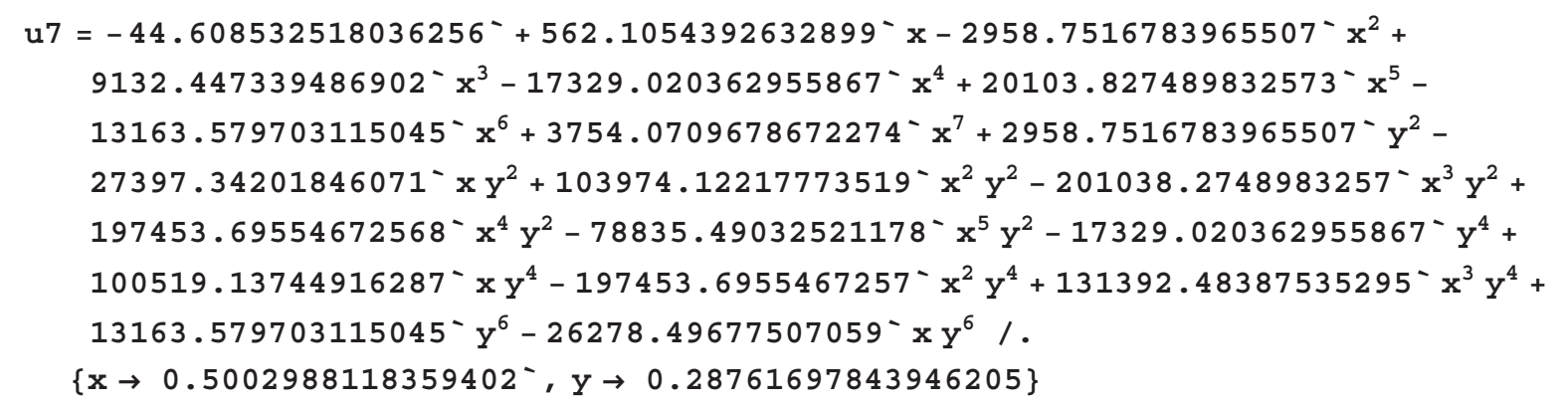

$\mathrm{v} 7=562.1054392632899^{-} \mathrm{y}-5917.503356793101^{-} \mathrm{x} y+27397.34201846071^{-} \mathrm{x}^{2} \mathrm{y}-$ $69316.08145182347 \mathrm{x}^{3} \mathrm{y}+100519.13744916285^{-} \mathrm{x}^{4} \mathrm{y}-78981.47821869029^{-} \mathrm{x}^{5} \mathrm{y}+$ $26278.49677507059^{-} x^{6} y-9132.447339486902^{-} y^{3}+69316.08145182347^{-} x y^{3}-$ $201038.27489832573^{-} x^{2} y^{3}+263271.59406230086^{-} x^{3} y^{3}-$ $131392.48387535295^{-} \mathrm{x}^{4} \mathrm{y}^{3}+20103.827489832573^{-} \mathrm{y}^{5}-78981.47821869027^{-} \mathrm{xy}^{5}+$ $78835.49032521178^{-} \mathrm{x}^{2} \mathrm{y}^{5}-3754.0709678672274^{-} \mathrm{y}^{7} \quad /$. $\left\{x \rightarrow 0.5002988118359402^{-}, y \rightarrow 0.28761697843946205\right\}$

2.89576

v7 /. $\quad\left\{x \rightarrow 0.29119665560733354^{-}, y^{\prime} \rightarrow 0.18592281926381385^{-}\right\}$

2.44792

v7 /. $\left\{x \rightarrow 0.29119665560733354^{-}, y^{\prime} \rightarrow-0.18592281926381385^{-}\right\}$

$-2.44792$

v7 /. $\left\{x->0.5002988118359402^{-}, y \rightarrow-0.28761697843946205^{-}\right\}$

$-2.89576$

$\mathrm{v7} / .\left\{\mathrm{x}->0.5002988118359402^{-}, \mathrm{y} \rightarrow 0.28761697843946205^{-}\right\}$

2.89576

$\mathrm{v} 7 / .\left\{\mathrm{x} \rightarrow 0.7112822388830826^{-}, \mathrm{y} \rightarrow 0.17942199664699282^{-}\right\}$

2.27478

gllambda $=\left(\operatorname{Sqrt}[13](-1)^{\wedge}(-j * 13)\right) /\left((\operatorname{Delta123} * \operatorname{Deltatil})^{\wedge}(1 / 4)\right) /$. $13 \rightarrow 0.5002988118359402^{-}+I * 0.28761697843946205$

$(1.97798-0.060729 \dot{\mathbb{i}})(-1)^{(-0.500299-0.287617}$ i) $j$

$\mathrm{Abs}\left[1.7993304340348035^{-}-14.23055382586453^{-}\right.$ii $]$

14.3439 


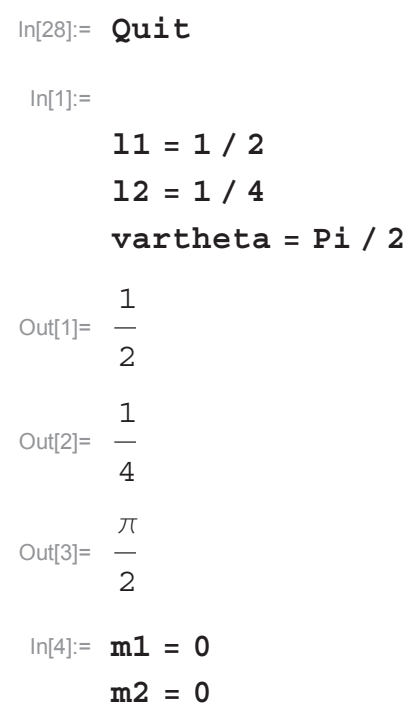


$\ln [11]:=$

h14 = Factor $\left[2 *\left(\operatorname{Sqrt}\left[(\operatorname{Delta123}) /\left(\operatorname{Delta} *\left(4-13^{\wedge} 2\right)\right)\right]\right)\right]$

Out[11]= $2 \sqrt{ }\left(\left(-9+156 \mathrm{x}^{2}-256 \mathrm{x}^{4}+312 \dot{i} \mathrm{x} y-1024 \dot{i} \mathrm{x}^{3} \mathrm{y}-156 \mathrm{y}^{2}+1536 \mathrm{x}^{2} \mathrm{y}^{2}+1024 \dot{\mathrm{i}} \mathrm{x} \mathrm{y}^{3}-256 \mathrm{y}^{4}\right) /\right.$

$\left(-36+649 x^{2}-1184 x^{4}+256 x^{6}+1298 \dot{i} x y-4736 \dot{i} x^{3} y+\right.$

$1536 \dot{i} x^{5} y-649 y^{2}+7104 x^{2} y^{2}-3840 x^{4} y^{2}+4736 \dot{i} x y^{3}-$

5120 i $\left.\left.x^{3} y^{3}-1184 y^{4}+3840 x^{2} y^{4}+1536 \dot{i} x y^{5}-256 y^{6}\right)\right)$

$\ln [12]:=$

h34 $=$ Factor $\left[(2 * \operatorname{Sqrt}[\operatorname{Delta123}]) /\left(12 * 13 * \operatorname{Sqrt}\left[\left(4-12^{\wedge} 2\right) *\left(4-13^{\wedge} 2\right)\right]\right)\right]$

$2 \sqrt{-9+156 x^{2}-256 x^{4}+312 \dot{i} x y-1024 \dot{i} x^{3} y-156 y^{2}+1536 x^{2} y^{2}+1024 \dot{i} x y^{3}-256 y^{4}}$

Out[12] $=\frac{2 \sqrt{7}(x+\dot{i} y) \sqrt{4-x^{2}-2 \dot{i} x y+y^{2}}}{3 \sqrt{ }}$

$\ln [13]:=$

h24 $=\operatorname{Factor}\left[(2 * \operatorname{Sqrt}[\operatorname{Delta123}]) /\left(11 * 13 * \operatorname{Sqrt}\left[\left(4-11^{\wedge} 2\right) *\left(4-13^{\wedge} 2\right)\right]\right)\right]$

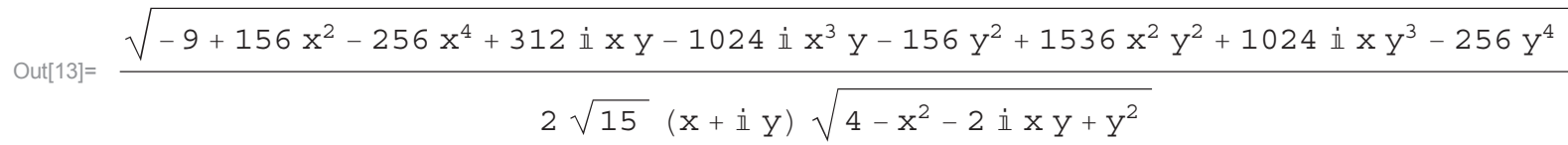

$\ln [14]:=$

h23 = Together $\left[(2 * \operatorname{Sqrt}[\operatorname{Delta123}]) /\left(11 * 12 * \operatorname{Sqrt}\left[\left(4-11^{\wedge} 2\right) *\left(4-12^{\wedge} 2\right)\right]\right)\right]$

$8 \sqrt{-9+156 x^{2}-256 x^{4}+312 \dot{i} x y-1024 \dot{i} x^{3} y-156 y^{2}+1536 x^{2} y^{2}+1024 \dot{i} x y^{3}-256 y^{4}}$ $3 \sqrt{105}$

$\ln [15]:=$

tetalambdal $=\operatorname{ArcCos}\left[\left(2 * 11^{\wedge} 2 *(\mathrm{~m} 1+\mathrm{m} 2)-\mathrm{m} 1 *\left(11^{\wedge} 2-12^{\wedge} 2+13^{\wedge} 2\right)\right) /\right.$

$\left.\left(\operatorname{Sqrt}\left[\left(\left(11^{\wedge} 2-\mathrm{ml}^{\wedge} 2\right) *\left(4 * 11^{\wedge} 2 * 13^{\wedge} 2-\left(11^{\wedge} 2-12^{\wedge} 2+13^{\wedge} 2\right)^{\wedge} 2\right)\right)\right]\right)\right]$

$\operatorname{Out}[15]=\frac{\pi}{2}$

$\ln [16]:=$

tetalambda2 $=\operatorname{ArcCos}\left[\left(2 * 12^{\wedge} 2 * \mathrm{~m} 1-\mathrm{m} 2 *\left(11^{\wedge} 2+12^{\wedge} 2-13^{\wedge} 2\right)\right) /\right.$

$\left.\left(\operatorname{Sgrt}\left[\left(\left(12^{\wedge} 2-\mathrm{m}^{\wedge} 2\right) *\left(4 * 11^{\wedge} 2 * 12^{\wedge} 2-\left(11^{\wedge} 2+12^{\wedge} 2-13^{\wedge} 2\right)^{\wedge} 2\right)\right)\right]\right)\right]$

$\operatorname{Out}[16]=\frac{\pi}{2}$

$\ln [17]:=$

tetalambda $3=\operatorname{ArcCos}\left[\left(2 * 13^{\wedge} 2 * m 2+(m 1+m 2) *\left(-11^{\wedge} 2+12^{\wedge} 2+13^{\wedge} 2\right)\right) /\right.$

$\left.\left(\operatorname{Sqrt}\left[\left(\left(13^{\wedge} 2-(\mathrm{m} 1+\mathrm{m} 2)^{\wedge} 2\right) *\left(4 * 12^{\wedge} 2 * 13^{\wedge} 2-\left(-11^{\wedge} 2+12^{\wedge} 2+13^{\wedge} 2\right)^{\wedge} 2\right)\right)\right]\right)\right]$

Out $[17]=\frac{\pi}{2}$

$\operatorname{In}[18]:=$ Deltatil $=$ Expand $\left[\left(11^{\wedge} 2-\mathrm{m}^{\wedge} 2\right) *\left(-11^{\wedge} 2+\mathrm{m} 1^{\wedge} 2+12^{\wedge} 2-\mathrm{m} 2^{\wedge} 2+13^{\wedge} 2-(\mathrm{m} 1+\mathrm{m} 2)^{\wedge} 2\right)+\right.$

$\left(12^{\wedge} 2-m 2^{\wedge} 2\right) *\left(11^{\wedge} 2-m 1^{\wedge} 2-12^{\wedge} 2+m 2^{\wedge} 2+13^{\wedge} 2-(m 1+m 2)^{\wedge} 2\right)+$

$\left.\left(13^{\wedge} 2-(m 1+m 2)^{\wedge} 2\right) *\left(11^{\wedge} 2-m 1^{\wedge} 2+12^{\wedge} 2-m 2^{\wedge} 2-13^{\wedge} 2+(m 1+m 2)^{\wedge} 2\right)\right]$

Out[18] $=-\frac{9}{256}+\frac{5 \mathrm{x}^{2}}{8}-\mathrm{x}^{4}+\frac{5 \dot{i} \mathrm{x} y}{4}-4 \dot{i} \mathrm{x}^{3} \mathrm{y}-\frac{5 \mathrm{y}^{2}}{8}+6 \mathrm{x}^{2} \mathrm{y}^{2}+4 \dot{\mathbf{i} x} \mathrm{y}^{3}-\mathrm{y}^{4}$ 
$\ln [19]:=$

$\mathrm{g} 1=((E) \wedge(I * P i * j * 13) * \operatorname{Sqrt}[13]) /$

$\left(\left(\text { Delta }-11^{\wedge} 2 * 12^{\wedge} 2 * 13^{\wedge} 2\right) *(\text { Deltatil })\right)^{\wedge}(1 / 4)$

$\operatorname{Out}[19]=\left(e^{i j \pi(x+\dot{i} y)} \sqrt{x+\dot{i} y}\right) /\left(\left(-\frac{9}{256}+\frac{5 x^{2}}{8}-x^{4}+\frac{5 \dot{i} x y}{4}-4 \dot{i} x^{3} y-\frac{5 y^{2}}{8}+6 x^{2} y^{2}+4 \dot{i} x y^{3}-y^{4}\right)\right.$

$\left.\left(-\frac{9}{256}+\frac{5 x^{2}}{8}-x^{4}-\frac{1}{64}(x+\dot{i} y)^{2}+\frac{5 \dot{i} x y}{4}-4 \dot{i} x^{3} y-\frac{5 y^{2}}{8}+6 x^{2} y^{2}+4 \dot{i} x y^{3}-y^{4}\right)\right)^{1 / 4}$ 



\section{Referências Bibliográficas}

[1] G. Arfken. Mathematical methodos for physicists. Academic press, inc, 1985. (Citado na página 133.)

[2] F. A. Berezin. General concept of quantization. Math. Phys., 40, 1975. (Citado na página 36.$)$

[3] F. A. Berezin. Quantization. Math. USSR Izvest., 8, 1975. (Citado na página 36.)

[4] F. A. Berezin. Quantization in complex symmetric spaces. Math. USSR Izvest., 9, 1975. (Citado na página 36.)

[5] L. C. Biedenharn and J. D Louck. Angular Momentum in Quantum Physics, theory and application. Addinson-Wesley Publishing Company, 1981. (Citados nas páginas 1, 9, 10, 13, 25 and 35.)

[6] L. C. Biedenharn and J. D Louck. The Racah-Wigner Algebra in Quantum Theory. Addinson-Wesley Publishing Company, 1981. (Citados nas páginas 23, 30 and 31.)

[7] M. Born, W. Heisenberg, and P. Jordan. Zur quantenmechanik ii. Z. Physik, 35:557-615, 1926. (Citado na página 21.)

[8] Siegmund Brandt and Hans Dieter Dahmen. The Picture Book of Quantum Mechanics. Springer-Velarg, 1995. (Citados nas páginas 1, 17, 18 and 19.)

[9] E. P. Wigner (Translated by J. J. Griffin). Group Theory and its Application to the Quantum Mechanics of Spectra. Academy Press, New York, 1959. (Citado na página 3.) 
[10] E. Cartan. Sur la structure des groupes finis and continus. Theses, Paris (Ouevres Complète), pages 137-287, 1984. (Citado na página 21.)

[11] A. Cayley. On the correspondence of homographies and rotations. Math. Ann., 15:238-240, 1879. (Citados nas páginas 10 and 11.)

[12] Pedro de M. Rios and Eldar Straume. Symbol correspondence for spin systems. Preprint. (Citados nas páginas 7, 8, 9, 10, 17, 18, 19, 33, 34, 35, 37, 39, 41, 66, $67,77,81$ and 82.)

[13] P.P. de M. Rios and A.M Ozorio de Almeida. On the propagation of semiclassical wigner functions. Journal of Physics A: Mathematical and General, 2002. (Citado na página 67.)

[14] A. R. Edmonds. Angular Momentum in Quantum Mechanics. Princenton, new jersey, princenton university press, 1960. (Citado na página 1.)

[15] F. Klein. Ueber binare formen mit linearem transformationen in sich selbst. Math. Ann., 9, 1875. (Citado na página 10.)

[16] F. Klein (Republication of the English translation (1913 by G. G Morrice) of Klein's book published in 1884.). The Icosahedron. Dover, New York, 1956. (Citado na página 10.)

[17] G. Ponzano and T. Regge. Semiclassical limit of racah coefficients. Spectroscopic and group theoretical methods in physics, North Holland Publ. Co., Amsterdan,, 1968. (Citados nas páginas 20, 30, 32, 68, 78, 97 and 99.)

[18] G. Racah. Theory of complex spectra i, ii, iii, iv. 61, 186(1942); 62, 438 (1942); 63, 367(1943);76,1352(1949). Phys. Rev. (Citado na página 30.)

[19] R.L. Stratonovich. On distributions in representations space. Soviet Physics Jetp, 4(6):891-898, 1957. (Citados nas páginas 34 and 37.)

[20] J. C. Várilly and J. M. Gracia-Bondía. The moyal representation for spin. Annals of physics, 190:107-148, 1989. (Citados nas páginas 19, 33, 34, 37 and 39.)

[21] D. A. Varshalovich, A. N. Moskalev, and V. K. Khersonskii. Quantum Theory of Angular Momentum: Irreducible Tensors, Spherical Harmonics, Vector Coupling Coefficients, 3nj Symbols. World Scientific, 1988. (Citados nas páginas 32 and 71.) 
[22] H. Weyl. The Classical Groups, their Invariants and Representation. Princeton Univ. Press, 1946. (Citados nas páginas 42 and 47.)

[23] E. P. Wigner. On the matrices with reduce the kronecker produtcs of representations of s. r. groups. Am. J. Math, 67, 1941. (Citado na página 30.) 